[Supporting Information]

\title{
Synthesis of Aryl Amine Derivatives from Benzyl Nitriles via Electrocyclization of in Situ Generated $N$-Silyl Ketene Imines
}

\author{
Fumihiko Yoshimura, ${ }^{\dagger} *$ Taiki Abe ${ }^{\ddagger}$ Keiji Tanino $^{\dagger} * *$ \\ ${ }^{\dagger}$ Department of Chemistry, Faculty of Science, Hokkaido University, Sapporo 060-0810, Japan \\ * Graduate School of Chemical Sciences and Engineering, Hokkaido University, Sapporo \\ 060-0810, Japan
}

\section{Table of Contents}

General Information

Optimization of the Cyclization of Nitrile 9 (Tables S1 and S2)

Experimental and Characterization Details

Preparation of Benzyl Nitriles 9, 12a-i, and 14a-f

S4 S16

Synthesis of Aryl Amines 10, 11, 13a-e, and 13g-i (Schemes 4 and 5)

S16 S22

Synthesis of Aryl Amines 15a-f (Scheme 7)

S22 S25

Synthesis of Steroidal Aryl Amine 19 (Scheme 8)

$\mathrm{S} 25 \sim \mathrm{S} 27$

Synthesis of Aryl Amine 22 (Reference 11)

S28 S29

Synthesis of Hydroxy Naphthylamine Derivative (Scheme 6)

S29 S30

Synthesis of Aryl Amines 10a and 10b (Table S2)

References

${ }^{1} \mathrm{H}$ and ${ }^{13} \mathrm{C}$ NMR Spectra

Benzyl Nitriles 9, 12a-i, and 14a-f

S32 S77

Aryl Amines 10, 11, 13a-e, and 13g-i (Schemes 4 and 5)

S78 S99

Aryl Amines 15a-f (Scheme 7)

S100 S114

Steroidal Aryl Amine 19 (Scheme 8)

$\mathrm{S} 115 \sim \mathrm{S} 120$

Nitrile 20 and Aryl Amines 21 and 22 (Reference 11)

S121 S126

Hydroxy Naphthylamine 13j (Scheme 6)

S127 S128

Aryl Amines 10a and 10b (Table S2)

S129 S132

${ }^{1} \mathrm{H}-{ }^{1} \mathrm{H}$ COSY and NOE Spectra of $\mathbf{1 5 c}$
S106 S108 


\section{General Information}

The reactions were performed using flame-dried glasswares under a positive pressure of argon. Tetrahydrofuran (THF) was distilled from sodium benzophenone ketyl. Anhydrous 1,2-dichloroethane, toluene, and dichloromethane were purchased from Sigma-Aldrich Co. Ltd or Kanto Chemical Co. Diisopropylamine, diisopropylethylamine and triethylamine was distilled from $\mathrm{CaH}_{2}$ under argon and stored in the presence of $\mathrm{NaOH}$ (pellets). All other reagents and solvents were used as received from commercial sources without further purification.

${ }^{1} \mathrm{H}$ NMR spectra were measured using a JEOL ECA-500 (500 MHz) in $\mathrm{CDCl}_{3}\left(\delta_{\mathrm{H}} 7.26\right), \mathrm{CD}_{3} \mathrm{OD}$ $\left(\delta_{\mathrm{H}} 3.30\right), \mathrm{CD}_{3} \mathrm{CN}\left(\delta_{\mathrm{H}} 1.93\right),\left(\mathrm{CD}_{3}\right)_{2} \mathrm{SO}\left(\delta_{\mathrm{H}} 2.49\right)$, and $\left(\mathrm{CD}_{3}\right)_{2} \mathrm{CO}\left(\delta_{\mathrm{H}} 2.04\right)$ with tetramethylsilane as an internal standard. Chemical shifts are reported in parts per million (ppm) from internal tetramethylsilane, and signal are expressed as singlet (s), doublet (d), triplet (t), quartet (q), septet (sept), and multiplet (m). Coupling constants are reported in $\mathrm{Hz} .{ }^{13} \mathrm{C}$ NMR spectra were measured using a JEOL ECA-500 (125 MHz) in $\mathrm{CDCl}_{3}\left(\delta_{\mathrm{C}} 77.0\right), \mathrm{CD}_{3} \mathrm{CN}\left(\delta_{\mathrm{C}} 118.2\right),\left(\mathrm{CD}_{3}\right)_{2} \mathrm{SO}\left(\delta_{\mathrm{C}} 39.7\right)$, $\left(\mathrm{CD}_{3}\right)_{2} \mathrm{CO}\left(\delta_{\mathrm{C}} 29.8\right), \mathrm{CD}_{3} \mathrm{OD}\left(\delta_{\mathrm{C}} 49.0\right)$ and $\mathrm{C}_{6} \mathrm{D}_{6}\left(\delta_{\mathrm{C}} 128.0\right)$ with tetramethylsilane as an internal standard. High-resolution mass spectra (HRMS) were recorded on a JEOL JMS-T100GCV or a JEOL JMS-SX102A at the GC-MS \& NMR Laboratory, Faculty of Agriculture, Hokkaido University. Infrared (IR) spectra were recorded on a JASCO FT/IR-4100 spectrophotometer. Analytical thin layer chromatography (TLC) was performed using $0.25 \mathrm{~mm}$ E. Merck Silica gel (60F-254) plates. Reaction components were visualized by illumination with ultraviolet light (254 $\mathrm{nm}$ ) and by staining with $6 \%$ ethanolic $p$-anisaldehyde (includes $6 \%$ conc. sulfuric acid and $1 \%$ acetic acid), $8 \%$ ethanolic phosphomolybdic acid, ceric ammonium molybdate in $10 \%$ sulfuric acid, or basic potassium permanganate solution. Kanto Chem. Co. Silica Gel 60N (particle size 0.040-0.050 mm) was used for flash column chromatography. To remove organotin impurities, $10 \mathrm{wt} \% \mathrm{~K}_{2} \mathrm{CO}_{3} / \mathrm{SiO}_{2}$ was used for flash column chromatography of the Stille coupling products. ${ }^{1}$ 


\section{Optimization of the Cyclization of Nitrile 9}

Table S1. Optimization of the aromatization of nitrile 9 under non-basic conditions<smiles>C=Cc1ccccc1C(C)OC</smiles>

9

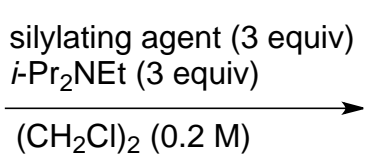

$\left(\mathrm{CH}_{2} \mathrm{Cl}\right)_{2}(0.2 \mathrm{M})$

10: $\mathrm{R}^{1}=\mathrm{H}$

11: $\mathrm{R}^{1}=\mathrm{COCF}_{3}$

\begin{tabular}{|c|c|c|c|c|}
\hline entry & silylating agent & temp. & time & results ${ }^{a}$ \\
\hline 1 & TESOTf & $85^{\circ} \mathrm{C}$ & $9.5 \mathrm{~h}$ & $10(1 \%), 9(79 \%)$ \\
\hline 2 & TMSOTf & $85^{\circ} \mathrm{C}$ & $9.5 \mathrm{~h}$ & $10(1 \%), 9$ (97\%) \\
\hline 3 & $\mathrm{TMSNTf}_{2}$ & $\mathrm{rt}$ & $1.5 \mathrm{~h}$ & $10(88 \%)$ \\
\hline 4 & $\mathrm{TMSNTf}_{2}{ }^{b}$ & $\mathrm{rt}$ & $1.5 \mathrm{~h}$ & 10 (7\%), 9 (57\%) \\
\hline 5 & $\mathrm{TMSNTf}_{2}{ }^{\mathrm{C}}$ & rt to $85^{\circ} \mathrm{C}$ & $1.5 \mathrm{~h}$ & $10(8 \%), 9(58 \%)$ \\
\hline 6 & $\mathrm{TMSNTf}_{2}{ }^{d}$ & rt to $85^{\circ} \mathrm{C}$ & $2 \mathrm{~h}$ & $10(22 \%), 9(50 \%)$ \\
\hline 7 & $\mathrm{TMSNTf}_{2}{ }^{e}$ & rt to $85^{\circ} \mathrm{C}$ & $2 \mathrm{~h}$ & $10(81 \%)$ \\
\hline 8 & $\mathrm{TMSNTf}_{2} e, f$ & rt to $85^{\circ} \mathrm{C}$ & $2 \mathrm{~h}$ & $10(9 \%), 9(45 \%)$ \\
\hline 9 & none & $85^{\circ} \mathrm{C}$ & $7 \mathrm{~h}$ & 9 (98\%) \\
\hline 10 & $\mathrm{TMSNTf}_{2}$ & $\mathrm{rt}$ & $1.5 \mathrm{~h}$ & $11(84 \%)^{g}$ \\
\hline
\end{tabular}

a Determined by ${ }^{1} \mathrm{H}$ NMR analysis of the crude product mixture using pyrazine as internal standard.

${ }^{b} \mathrm{Et}_{3} \mathrm{~N}$ was used instead of $i-\mathrm{Pr}_{2} \mathrm{NEt}$.

$c$ TMSNTf $_{2}$ (1 equiv) and $i-\mathrm{Pr}_{2} \mathrm{NEt}$ (1 equiv) were used.

${ }^{d} \mathrm{TMSNTf}_{2}$ (2 equiv) and $i-\mathrm{Pr}_{2} \mathrm{NEt}$ (2 equiv) were used.

$e$ In absence of $i$ - $\operatorname{Pr}_{2}$ NEt.

${ }^{f}$ Catalytic amout of $\mathrm{TMSNTf}_{2}$ (0.3 equiv) was used.

$g$ Isolated yield after amidation with TFAA (2 equiv) in one-pot.

Table S2. Optimization of the aromatization of nitrile 9 under basic anionic conditions

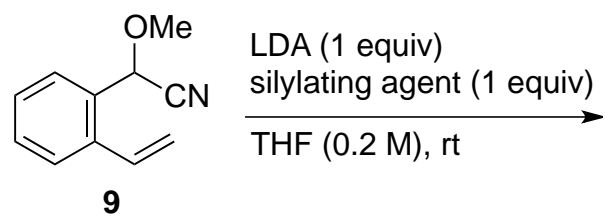<smiles>CCNc1ccc2ccccc2c1OC</smiles>

10a: $R^{1}=T B S, 10 b: R^{1}=$ TIPS

11: $\mathrm{R}^{1}=\mathrm{COCF}_{3}$

\begin{tabular}{ccll}
\hline entry & silylating agent & time & \multicolumn{1}{c}{ results $^{a}$} \\
\hline 1 & TBSCl & $1 \mathrm{~h}$ & $\mathbf{1 0 a}(59 \%), \mathbf{9}(40 \%)$ \\
2 & TIPSCI & $1 \mathrm{~h}$ & $\mathbf{1 0 b}(87 \%)$ \\
3 & none & $2 \mathrm{~h}$ & $\mathbf{9}(9 \%)+$ unidentified products \\
4 & TIPSCI $^{b}$ & $1.5 \mathrm{~h}$ & $\mathbf{9}(82 \%)$ \\
5 & TIPSCI & $1 \mathrm{~h}$ & $\mathbf{1 1}(80 \%, 2 \text { steps })^{c}$ \\
\hline
\end{tabular}

a Determined by ${ }^{1} \mathrm{H}$ NMR analysis of the crude product mixture using pyrazine as internal standard.

$b$ In absence of LDA.

$c 1.1$ equivalent of LDA was used. Isolated yield after amidation with TFAA (2 equiv). 


\section{Experimental Details}

\section{Preparation of Benzyl Nitriles 9, 12a-i, and 14a-f}

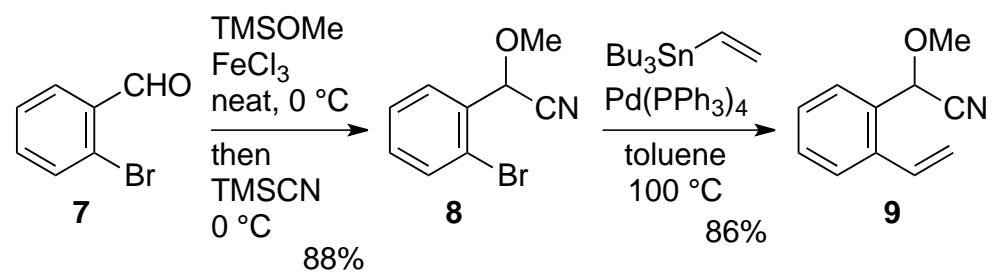

2-(2-Bromophenyl)-2-methoxyacetonitrile (8): To a mixture of methoxytrimethylsilane (1.3 mL, $9.6 \mathrm{mmol})$ and $\mathrm{FeCl}_{3}(13.0 \mathrm{mg}, 0.080 \mathrm{mmol})$ was added 2-bromobenzaldehyde (7) (467 $\mu \mathrm{L}, 4.0$ mmol) and the mixture was stirred at $0{ }^{\circ} \mathrm{C}$ for $2 \mathrm{~h}$. Trimethylsilyl cyanide $(751 \mu \mathrm{L}, 6.0 \mathrm{mmol})$ was added to the mixture, and the resulting mixture was stirred at $0{ }^{\circ} \mathrm{C}$ for $80 \mathrm{~min}$. Saturated aqueous sodium bicarbonate (ca. $3 \mathrm{~mL}$ ) was added to the mixture and the products were extracted with $\mathrm{CH}_{2} \mathrm{Cl}_{2}$. The combined organic layers were dried over $\mathrm{MgSO}_{4}$ and concentrated under reduced pressure. Purification by flash column chromatography $\left(\mathrm{SiO}_{2}\right.$, hexane- $\left.\mathrm{Et}_{2} \mathrm{O}=5: 1\right)$ afforded cyanohydrin methyl ether 8 (795.8 mg, $3.52 \mathrm{mmol}, 88 \%)$.

Colorless oil; ${ }^{1} \mathrm{H}$ NMR $\left(500 \mathrm{MHz}, \mathrm{CDCl}_{3}\right) \delta 7.70(1 \mathrm{H}, \mathrm{dd}, J=8.0,1.7 \mathrm{~Hz}), 7.62(1 \mathrm{H}, \mathrm{d}, J=8.1$ $\mathrm{Hz}), 7.44-7.38(1 \mathrm{H}, \mathrm{m}), 7.32-7.27(1 \mathrm{H}, \mathrm{m}), 5.44(1 \mathrm{H}, \mathrm{s}), 3.62(3 \mathrm{H}, \mathrm{s}) ;{ }^{13} \mathrm{C} \mathrm{NMR}(125 \mathrm{MHz}$, $\left.\mathrm{CDCl}_{3}\right) \delta 133.11,132.71,131.23,128.92,127.99,122.83,116.31,71.63,57.96$; IR (ATR) v 2394, 2830, 1572, 1470, 1437, 1323, 1277, 1198, 1083, 1026, 972, 947, 751, $632 \mathrm{~cm}^{-1}$; HRMS (FD) calcd for $\mathrm{C}_{9} \mathrm{H}_{8} \mathrm{BrNO}\left(\mathrm{M}^{+}\right)$: 224.9789, found: 224.9776 .

2-Methoxy-2-(2-vinylphenyl)acetonitrile (9): To a mixture of cyanohydrin methyl ether 8 (866 $\mathrm{mg}, 774 \mu \mathrm{L}, 5.0 \mathrm{mmol})$ and tributyl(vinyl)stannane $(1.8 \mathrm{~mL}, 6.0 \mathrm{mmol})$ in toluene $(12.5 \mathrm{~mL})$ was added $\mathrm{Pd}\left(\mathrm{PPh}_{3}\right)_{4}(231.1 \mathrm{mg}, 0.20 \mathrm{mmol})$ and the mixture was stirred at $100{ }^{\circ} \mathrm{C}$ for $18 \mathrm{~h}$. The mixture was filtered through a pad of Celite and the filtrate was concentrated under reduced pressure. Purification by flash column chromatography $\left(10 \mathrm{wt} \% \mathrm{~K}_{2} \mathrm{CO}_{3} / \mathrm{SiO}_{2}\right.$, hexane- $\left.\mathrm{Et}_{2} \mathrm{O}=15: 1\right)$ afforded nitrile 9 (746.8 $\mathrm{mg}, 4.31 \mathrm{mmol}, 86 \%)$.

Colorless oil; ${ }^{1} \mathrm{H}$ NMR $\left(500 \mathrm{MHz}, \mathrm{CDCl}_{3}\right) \delta 7.60(1 \mathrm{H}, \mathrm{d}, J=7.6 \mathrm{~Hz}), 7.54(1 \mathrm{H}, \mathrm{d}, J=8.0 \mathrm{~Hz})$, $7.42(1 \mathrm{H}, \mathrm{t}, J=7.5 \mathrm{~Hz}), 7.35(1 \mathrm{H}, \mathrm{t}, J=7.5 \mathrm{~Hz}), 6.97(1 \mathrm{H}, \mathrm{dd}, J=17.2,10.9 \mathrm{~Hz}), 5.71(1 \mathrm{H}, \mathrm{dd}, J$ $=17.2,1.2 \mathrm{~Hz}), 5.46(1 \mathrm{H}, \mathrm{d}, J=10.9 \mathrm{~Hz}), 5.38(1 \mathrm{H}, \mathrm{s}), 3.54(3 \mathrm{H}, \mathrm{s}) ;{ }^{13} \mathrm{C} \mathrm{NMR}(125 \mathrm{MHz}$, $\left.\mathrm{CD}_{3} \mathrm{OD}\right) \delta 138.56,134.23,131.91,131.13,129.16,129.10,127.73,118.29,118.25,71.28,57.53$; IR (ATR) v 3264, 2161, 1710, 1538, 1389, 1318, 1260, 1160, 1081, 1054, 813, $749 \mathrm{~cm}^{-1}$; HRMS (FD) calcd for $\mathrm{C}_{11} \mathrm{H}_{11} \mathrm{NO}\left(\mathrm{M}^{+}\right)$: 173.0841, found: 173.0848 . 


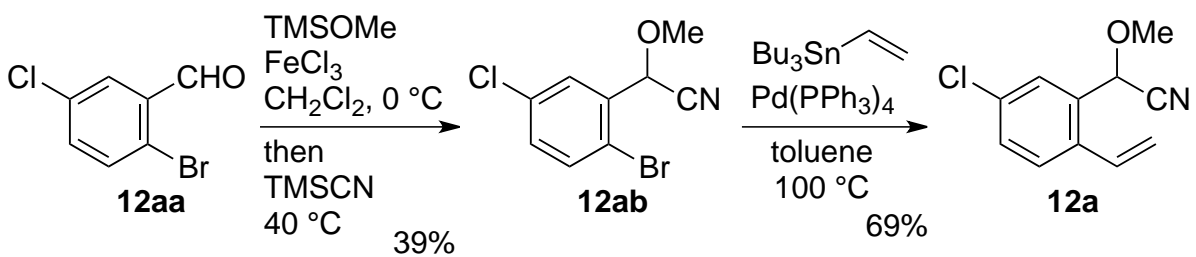

2-(2-Bromo-5-chlorophenyl)-2-methoxyacetonitrile

(12ab):

A mixture of methoxytrimethylsilane (3.3 mL, $24 \mathrm{mmol}$ ), 2-bromo-5-chlorobenzaldehyde (12aa) (2.19 g, 10 mmol), and $\mathrm{FeCl}_{3}(32.4 \mathrm{mg}, 0.20 \mathrm{mmol})$ in $\mathrm{CH}_{2} \mathrm{Cl}_{2}(5 \mathrm{~mL})$ was stirred at $0{ }^{\circ} \mathrm{C}$ for $3 \mathrm{~h}$. Trimethylsilyl cyanide $(1.9 \mathrm{~mL}, 15 \mathrm{mmol})$ was then added and the mixture was stirred at $40{ }^{\circ} \mathrm{C}$ for $18 \mathrm{~h}$. Saturated aqueous sodium bicarbonate (ca. $10 \mathrm{~mL}$ ) was added to the mixture and the products were extracted with $\mathrm{CH}_{2} \mathrm{Cl}_{2}$. The combined organic layers were dried over $\mathrm{MgSO}_{4}$ and concentrated under reduced pressure. Purification by flash column chromatography $\left(\mathrm{SiO}_{2}\right.$, hexane- $\left.\mathrm{Et}_{2} \mathrm{O}=20: 1\right)$ afforded cyanohydrin methyl ether 12ab (1.02 g, $\left.3.92 \mathrm{mmol}, 39 \%\right)$.

White solid; M.p. $108-109{ }^{\circ} \mathrm{C} ;{ }^{1} \mathrm{H}$ NMR $\left(500 \mathrm{MHz}, \mathrm{CDCl}_{3}\right) \delta 7.68(1 \mathrm{H}, \mathrm{d}, J=2.3 \mathrm{~Hz}), 7.54(1 \mathrm{H}$, $\mathrm{d}, J=8.1 \mathrm{~Hz}), 7.28(1 \mathrm{H}, \mathrm{dd}, J=8.6,2.9 \mathrm{~Hz}), 5.35(1 \mathrm{H}, \mathrm{s}), 3.64(3 \mathrm{H}, \mathrm{s}) ;{ }^{13} \mathrm{C} \mathrm{NMR}(125 \mathrm{MHz}$, $\left.\mathrm{CDCl}_{3}\right) \delta 134.50,134.40,134.21,131.30,128.98,120.31,115.78,71.21,58.29$; IR (ATR) v 3095, 2952, 2833, 2065, 1455, 1194, 1091, 970, 866, $818 \mathrm{~cm}^{-1}$; HRMS (FD) calcd for $\mathrm{C}_{9} \mathrm{H}_{7} \mathrm{BrClNO}$ $\left(\mathrm{M}^{+}\right)$: 258.9400, found: 258.9396 .

2-(5-Chloro-2-vinylphenyl)-2-methoxyacetonitrile (12a): To a mixture of cyanohydrin methyl ether 12ab (781.5 mg, $3 \mathrm{mmol})$ and tributyl(vinyl)stannane $(994 \mu \mathrm{L}, 3.3 \mathrm{mmol})$ in toluene (7.5 $\mathrm{mL}$ ) was added $\mathrm{Pd}\left(\mathrm{PPh}_{3}\right)_{4}(138.7 \mathrm{mg}, 0.12 \mathrm{mmol})$ and the mixture was stirred at $100{ }^{\circ} \mathrm{C}$ for $16 \mathrm{~h}$. The mixture was filtered through a pad of Celite and the filtrate was concentrated under reduced pressure. Purification by flash column chromatography $\left(10 \mathrm{wt} \% \mathrm{~K}_{2} \mathrm{CO}_{3} / \mathrm{SiO}_{2}\right.$, hexane-EtOAc $=$ 10:1) afforded nitrile 9 (428.5 mg, $2.06 \mathrm{mmol}, 69 \%)$.

Colorless oil; ${ }^{1} \mathrm{H}$ NMR (500 MHz, $\left.\mathrm{CDCl}_{3}\right) \delta 7.59(1 \mathrm{H}, \mathrm{d}, J=2.3 \mathrm{~Hz}), 7.47(1 \mathrm{H}, \mathrm{d}, J=8.6 \mathrm{~Hz})$, $7.38(1 \mathrm{H}, \mathrm{dd}, J=8.6,1.8 \mathrm{~Hz}), 6.89(1 \mathrm{H}, \mathrm{dd}, J=17.2,10.9 \mathrm{~Hz}), 5.70(1 \mathrm{H}, \mathrm{d}, J=17.2 \mathrm{~Hz}), 5.48$ $(1 \mathrm{H}, \mathrm{d}, J=10.9 \mathrm{~Hz}), 5.30(1 \mathrm{H}, \mathrm{s}), 3.56(3 \mathrm{H}, \mathrm{s}) ;{ }^{13} \mathrm{C} \mathrm{NMR}\left(125 \mathrm{MHz}, \mathrm{CDCl}_{3}\right) \delta 135.51,133.93$, 131.66, 131.53, 130.16, 128.30, 127.89, 119.23, 116.22, 69.59, 57.41; IR (ATR) v 2934, 2830, 1960, 1595, 1481, 1190, 973, 932, $832 \mathrm{~cm}^{-1}$; HRMS (FD) calcd for $\mathrm{C}_{9} \mathrm{H}_{7} \mathrm{BrClNO}\left(\mathrm{M}^{+}\right)$: 258.9400 , found: 258.9396.

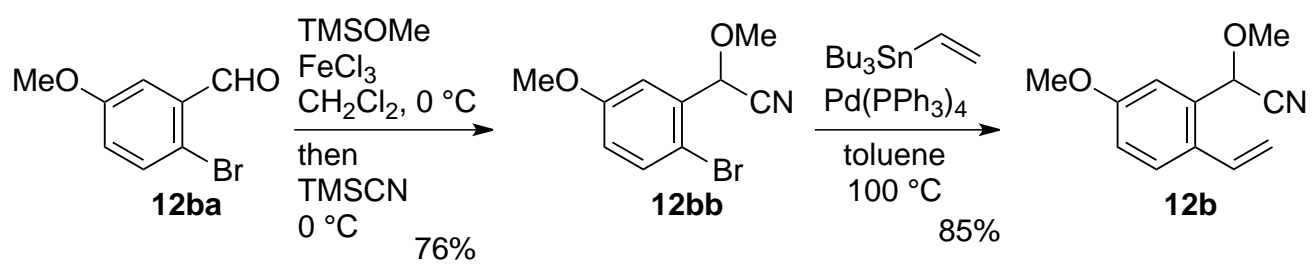

2-(2-Bromo-5-methoxyphenyl)-2-methoxyacetonitrile

(12bb): A mixture of 
2-bromo-5-methoxybenzaldehyde (12ba) $(856.6 \mathrm{mg}, 4.0 \mathrm{mmol})$, methoxytrimethylsilane $(1.3 \mathrm{~mL}$, $9.6 \mathrm{mmol})$ and $\mathrm{FeCl}_{3}(13.0 \mathrm{mg}, 0.08 \mathrm{mmol})$ in $\mathrm{CH}_{2} \mathrm{Cl}_{2}(2 \mathrm{~mL})$ was stirred at $0{ }^{\circ} \mathrm{C}$ for $3 \mathrm{~h}$. Trimethylsilyl cyanide $(751 \mu \mathrm{L}, 6.0 \mathrm{mmol})$ was then added and the mixture was stirred at $0{ }^{\circ} \mathrm{C}$ for $1 \mathrm{~h}$. Saturated aqueous sodium bicarbonate (ca. $3 \mathrm{~mL}$ ) was added to the mixture and the products were extracted with $\mathrm{CH}_{2} \mathrm{Cl}_{2}$. The combined organic layers were dried over $\mathrm{MgSO}_{4}$ and concentrated under reduced pressure. Purification by flash column chromatography $\left(\mathrm{SiO}_{2}\right.$, hexane-EtOAc $=15: 1)$ afforded cyanohydrin methyl ether $\mathbf{1 2 b b}(774.9 \mathrm{mg}, 3.03 \mathrm{mmol}, 76 \%)$.

White solid; M.p. 67-68 ${ }^{\circ} \mathrm{C}$; ${ }^{1} \mathrm{H}$ NMR $\left(500 \mathrm{MHz}, \mathrm{CDCl}_{3}\right) \delta 7.48(1 \mathrm{H}, \mathrm{d}, J=9.2 \mathrm{~Hz}), 7.22(1 \mathrm{H}, \mathrm{d}$, $J=2.9 \mathrm{~Hz}), 6.84(1 \mathrm{H}, \mathrm{dd}, J=9.2,2.9 \mathrm{~Hz}), 5.38(1 \mathrm{H}, \mathrm{s}), 3.83(3 \mathrm{H}, \mathrm{s}), 3.62(3 \mathrm{H}, \mathrm{s}) ;{ }^{13} \mathrm{C} \mathrm{NMR}(125$ $\left.\mathrm{MHz}, \mathrm{CD}_{3} \mathrm{OD}\right) \delta 159.35,133.75,133.56,117.34,116.32,114.10,112.76,71.63,58.07,55.61$; IR $(\mathrm{ATR}) \vee 2950,2830,2161,1577,1471,1295,1229,1089,1052,968,861,771,598 \mathrm{~cm}^{-1}$; HRMS (FD) calcd for $\mathrm{C}_{11} \mathrm{H}_{10} \mathrm{ClNO}\left(\mathrm{M}^{+}\right)$: 207.0451, found: 207.0462.

2-Methoxy-2-(5-methoxy-2-vinylphenyl)acetonitrile (12b): To a solution of cyanohydrin methyl ether $12 \mathrm{bb}(774.9 \mathrm{mg}, 3.03 \mathrm{mmol})$ and tributyl(vinyl)stannane $(1.0 \mathrm{~mL}, 3.3 \mathrm{mmol})$ in toluene $(7.5$ $\mathrm{mL}$ ) was added $\mathrm{Pd}\left(\mathrm{PPh}_{3}\right)_{4}(139.8 \mathrm{mg}, 0.12 \mathrm{mmol})$ and the mixture was stirred at $100{ }^{\circ} \mathrm{C}$ for $30 \mathrm{~h}$. The mixture was filtered through a pad of Celite and the filtrate was concentrated under reduced pressure. Purification by flash column chromatography $\left(10 \mathrm{wt} \% \mathrm{~K}_{2} \mathrm{CO}_{3} / \mathrm{SiO}_{2}\right.$, hexane-EtOAc $=$ 10:1) afforded nitrile $\mathbf{1 2 b}(524.6 \mathrm{mg}, 2.58 \mathrm{mmol}, 85 \%)$.

White solid; M.p. 44-45 ${ }^{\circ} \mathrm{C}$; ${ }^{1} \mathrm{H}$ NMR $\left(500 \mathrm{MHz}, \mathrm{CDCl}_{3}\right) \delta 7.47(1 \mathrm{H}, \mathrm{d}, J=8.6 \mathrm{~Hz}), 7.13(1 \mathrm{H}, \mathrm{d}$, $J=2.9 \mathrm{~Hz}), 6.94(1 \mathrm{H}, \mathrm{dd}, J=8.6,2.9 \mathrm{~Hz}), 6.89(1 \mathrm{H}, \mathrm{dd}, J=17.2,10.9 \mathrm{~Hz}), 5.60(1 \mathrm{H}, \mathrm{dd}, J=17.2$, $1.1 \mathrm{~Hz}), 5.35(1 \mathrm{H}, \mathrm{d}, J=10.4 \mathrm{~Hz}), 5.34(1 \mathrm{H}, \mathrm{s}), 3.85(3 \mathrm{H}, \mathrm{s}), 3.55(3 \mathrm{H}, \mathrm{s}) ;{ }^{13} \mathrm{C}$ NMR $(125 \mathrm{MHz}$, $\left.\mathrm{CDCl}_{3}\right) \delta 160.95,133.63,133.17,130.79,129.05,118.27,116.43,116.11,114.30,71.13,57.60$, 55.90; IR (ATR) v 2952, 2163, 1610, 1494, 1297, 1239, 1073, 968, $862 \mathrm{~cm}^{-1}$; HRMS (FD) calcd for $\mathrm{C}_{12} \mathrm{H}_{13} \mathrm{NO}_{2}\left(\mathrm{M}^{+}\right)$: 203.0946, found: 203.0957.

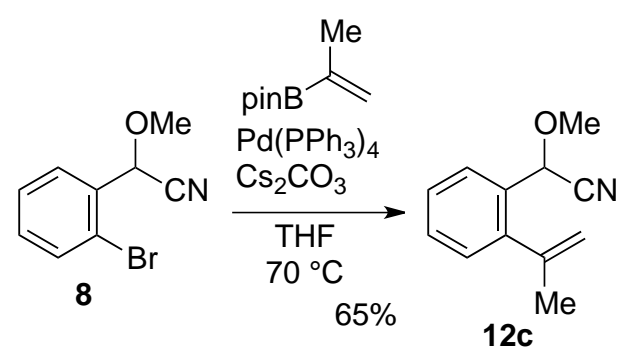

2-Methoxy-2-(2-(prop-1-en-2-yl)phenyl)acetonitrile (12c): To a mixture of cyanohydrin methyl ether 8 (346 mg, $310 \mu \mathrm{L}, 2 \mathrm{mmol}$ ), 2-isopropenyl-4,4,5,5-tetramethyl-1,3,2-dioxaborolane (752 $\mu \mathrm{L}$, $4 \mathrm{mmol})$, and $\mathrm{Cs}_{2} \mathrm{CO}_{3}(1.95 \mathrm{~g}, 6 \mathrm{mmol})$ in THF $(20 \mathrm{~mL})$ was added $\mathrm{Pd}\left(\mathrm{PPh}_{3}\right)_{4}(693.3 \mathrm{mg}, 0.6$ mmol) and the reaction mixture was stirred at $70{ }^{\circ} \mathrm{C}$ for $27 \mathrm{~h}$. The mixture was filtered through a pad of Celite and the filtrate was concentrated under reduced pressure. Purification by flash column chromatography $\left(\mathrm{SiO}_{2}\right.$, hexane-Et $\left.{ }_{2} \mathrm{O}=10: 1\right)$ afforded nitrile 12c $(243.0 \mathrm{mg}, 1.30 \mathrm{mmol}$, 
$65 \%)$.

Colorless oil; ${ }^{1} \mathrm{H}$ NMR $\left(500 \mathrm{MHz}, \mathrm{CDCl}_{3}\right) \delta 7.69(1 \mathrm{H}, \mathrm{dd}, J=7.5,1.7 \mathrm{~Hz}), 7.39(1 \mathrm{H}, \mathrm{td}, J=7.5$, $1.7 \mathrm{~Hz}), 7.36(1 \mathrm{H}, \mathrm{td}, J=7.5,1.7 \mathrm{~Hz}), 7.24(1 \mathrm{H}, \mathrm{dd}, J=9.7,2.1 \mathrm{~Hz}), 5.34(1 \mathrm{H}, \mathrm{s}), 5.33-5.31(1 \mathrm{H}$, m), $4.94(1 \mathrm{H}, \mathrm{s}), 3.53(3 \mathrm{H}, \mathrm{s}), 2.09(3 \mathrm{H}, \mathrm{s}) ;{ }^{13} \mathrm{C} \mathrm{NMR}\left(125 \mathrm{MHz}, \mathrm{CDCl}_{3}\right) \delta 143.44,143.17,130.30$, 129.61, 128.14, 128.06, 127.68, 117.68, 116.72, 69.70, 57.39, 25.13; IR (ATR) v 3073, 2944, $2828,2333,1639,1489,1448,1190,1077,970,910,756 \mathrm{~cm}^{-1}$; HRMS (FD) calcd for $\mathrm{C}_{12} \mathrm{H}_{13} \mathrm{NO}$ $\left(\mathrm{M}^{+}\right)$: 187.0997, found: 187.1009 .

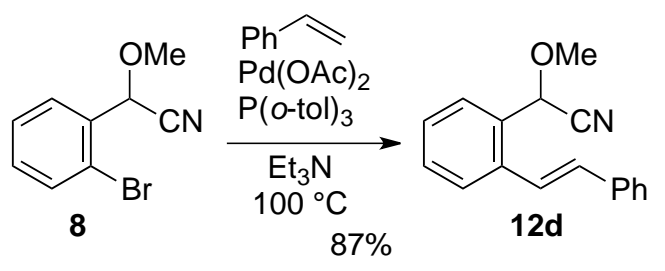

(E)-2-Methoxy-2-(2-styrylphenyl)acetonitrile (12d): A mixture of cyanohydrin methyl ether 8 (866 mg, $774 \mu \mathrm{L}, 5 \mathrm{mmol})$, stylene $(723 \mu \mathrm{L}, 6.3 \mathrm{mmol})$, tri (o-tolyl)phosphine (60.9 mg, $0.2 \mathrm{mmol})$, and $\mathrm{Pd}(\mathrm{OAc})_{2}(693.3 \mathrm{mg}, 0.6 \mathrm{mmol})$ in $\mathrm{Et}_{3} \mathrm{~N}(2.5 \mathrm{~mL})$ was stirred at $100{ }^{\circ} \mathrm{C}$ for $24 \mathrm{~h}$ in a sealed tube. The mixture was filtered through a pad of Celite and the filtrate was concentrated under reduced pressure. Purification by flash column chromatography $\left(\mathrm{SiO}_{2}\right.$, hexane- $\left.\mathrm{Et}_{2} \mathrm{O}=5: 1\right)$ afforded nitrile 12d (1.09 g, $4.37 \mathrm{mmol}, 87 \%)$.

Colorless oil; ${ }^{1} \mathrm{H}$ NMR (500 MHz, $\left.\mathrm{CDCl}_{3}\right) \delta 7.67(1 \mathrm{H}, \mathrm{d}, J=7.5 \mathrm{~Hz}), 7.63(1 \mathrm{H}, \mathrm{d}, J=7.5 \mathrm{~Hz})$, $7.53(2 \mathrm{H}, \mathrm{d}, J=7.5 \mathrm{~Hz}), 7.45(1 \mathrm{H}, \mathrm{t}, J=7.2 \mathrm{~Hz}), 7.42-7.29(5 \mathrm{H}, \mathrm{m}), 7.04(1 \mathrm{H}, \mathrm{d}, J=16.1 \mathrm{~Hz})$, $5.46(1 \mathrm{H}, \mathrm{s}), 3.57(3 \mathrm{H}, \mathrm{s}) ;{ }^{13} \mathrm{C} \mathrm{NMR}\left(125 \mathrm{MHz}, \mathrm{CDCl}_{3}\right) \delta 137.02,136.83,133.15,130.23,130.06$, 128.78, 128.42, 128.25, 127.88, 126.98, 126.79, 124.06, 116.80, 70.56, 57.09; IR (ATR) v 3028, 2932, 2827, 2122, 1496, 1450, 1189, 1079, 965, 761, $691 \mathrm{~cm}^{-1}$; HRMS (FD) calcd for $\mathrm{C}_{17} \mathrm{H}_{15} \mathrm{NO}$ $\left(\mathrm{M}^{+}\right)$: 249.1154, found: 249.1146 .

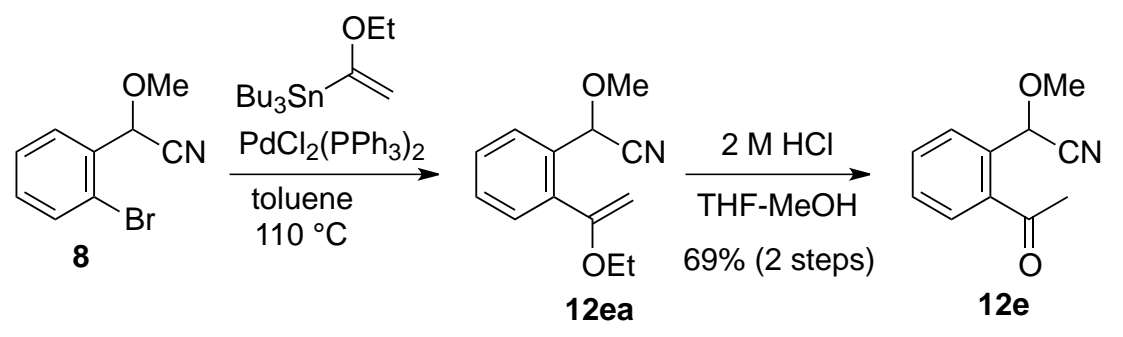

2-(2-Acetylphenyl)-2-methoxyacetonitrile (12e): To a solution of cyanohydrin methyl ether 8 (339 mg, $232 \mu \mathrm{L}, 1.50 \mathrm{mmol})$ and tributyl(1-ethoxyvinyl)stannane $(547 \mu \mathrm{L}, 1.65 \mathrm{mmol})$ in toluene $(0.6 \mathrm{~mL})$ was added $\mathrm{PdCl}_{2}\left(\mathrm{PPh}_{3}\right)_{2}(10.5 \mathrm{mg}, 0.015 \mathrm{mmol})$, and the mixture was stirred at $110{ }^{\circ} \mathrm{C}$ for $3 \mathrm{~h}$. The mixture was filtered through a pad of Celite and the filtrate was concentrated under reduced pressure to give vinyl ether 12ea $(923.2 \mathrm{mg})$. This product was used for the next step without further purification. 
To a solution of the above vinyl ether $12 \mathrm{ea}(923.2 \mathrm{mg}$, as $1.50 \mathrm{mmol})$ in THF (2 mL) and $\mathrm{MeOH}$ $(0.5 \mathrm{~mL})$ was added $2 \mathrm{M}$ aqueous $\mathrm{HCl}(1 \mathrm{~mL})$ and the mixture was stirred at room temperature for $1 \mathrm{~h}$. Saturated sodium bicarbonate $(2 \mathrm{~mL})$ was added to the mixture and the products were extracted with $\mathrm{Et}_{2} \mathrm{O}$. The combined organic layers were dried over $\mathrm{MgSO}_{4}$ and concentrated under reduced pressure. Purification by flash column chlomatography $\left(\mathrm{SiO}_{2}\right.$, hexane-EtOAc $\left.=1: 1\right)$ followed by recrystallization from hexane- $\mathrm{Et}_{2} \mathrm{O}$ afforded nitrile 12e $(194.8 \mathrm{mg}, 1.03 \mathrm{mmol}$, 69\% for 2 steps).

Yellow solid; M.p. 70-73 ${ }^{\circ} \mathrm{C}$ (hexane-Et $2 \mathrm{O}$ ); ${ }^{1} \mathrm{H}$ NMR $\left(500 \mathrm{MHz}, \mathrm{CDCl}_{3}\right) \delta$ 7.91-7.84 (2H, m), 7.65-7.59 (1H, m), 7.55-7.50 (1H, m), $5.95(1 \mathrm{H}, \mathrm{s}), 3.63(3 \mathrm{H}, \mathrm{s}), 2.65(3 \mathrm{H}, \mathrm{s}) ;{ }^{13} \mathrm{C}$ NMR $(125$ $\left.\mathrm{MHz}, \mathrm{CDCl}_{3}\right) \delta 200.65,135.11,133.97,132.81,130.20,129.37,127.87,117.26,69.48 .58 .27$, 28.79; IR (ATR) v 2939, 2833, 2174, 1672, 1576, 1360, 1260, 1092, 973, 770, $588 \mathrm{~cm}^{-1}$; HRMS (FD) calcd for $\mathrm{C}_{11} \mathrm{H}_{11} \mathrm{NO}_{2}\left(\mathrm{M}^{+}\right):$189.0790, found: 189.0794 .

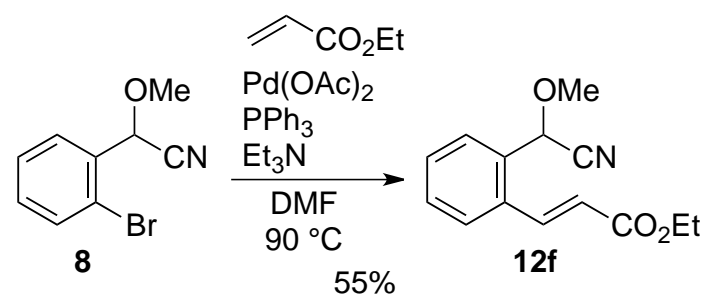

Ethyl (E)-3-(2-(cyano(methoxy)methyl)phenyl)acrylate (12f): To a solution of $\mathrm{Pd}(\mathrm{OAc})_{2}(11.2$ $\mathrm{mg}, 0.050 \mathrm{mmol})$ and triphenylphosphine $(26.2 \mathrm{mg}, 0.10 \mathrm{mmol})$ in DMF $(14 \mathrm{~mL})$ were added cyanohydrin methyl ether $8(866 \mathrm{mg}, 774 \mu \mathrm{L}, 5 \mathrm{mmol})$, ethyl acrylate $(1.6 \mathrm{~mL}, 15 \mathrm{mmol})$, and $\mathrm{Et}_{3} \mathrm{~N}(0.98 \mathrm{~mL}, 7.0 \mathrm{mmol})$. The mixture was stirred at $90{ }^{\circ} \mathrm{C}$ for $15 \mathrm{~h}$. The reaction mixture was filtered through a pad of Celite and the filtrate was concentrated under reduced pressure. Purification by flash column chromatography $\left(\mathrm{SiO}_{2}\right.$, hexane-EtOAc $\left.=3: 1\right)$ afforded nitrile $\mathbf{1 2 f}$ (680.0 mg, $2.77 \mathrm{mmol}, 55 \%)$.

Colorless oil; ${ }^{1} \mathrm{H}$ NMR $\left(500 \mathrm{MHz}, \mathrm{CDCl}_{3}\right) \delta 7.92(1 \mathrm{H}, \mathrm{d}, J=16.1 \mathrm{~Hz}), 7.72-7.66(1 \mathrm{H}, \mathrm{m})$, 7.65-7.60 (1H, m), 7.49-7.43 (2H, m), $6.40(1 \mathrm{H}, \mathrm{d}, J=16.1 \mathrm{~Hz}), 5.44(1 \mathrm{H}, \mathrm{s}), 4.29(2 \mathrm{H}, \mathrm{q}, J=6.9$ $\mathrm{Hz}), 3.58(3 \mathrm{H}, \mathrm{s}), 1.35(3 \mathrm{H}, \mathrm{t}, J=6.9 \mathrm{~Hz}) ;{ }^{13} \mathrm{C} \mathrm{NMR}\left(125 \mathrm{MHz}, \mathrm{CDCl}_{3}\right) \delta 166.15,139.72,133.77$, $131.46,130.31,130.13,128.49,127.52,122.22,116.32,70.10,60.70,57.27,14.19$; IR (ATR) $v$ 2988, 2902, 2041, 1715, 1483, 1366, 1267, 1074, 969, $763 \mathrm{~cm}^{-1}$; HRMS (FD) calcd for $\mathrm{C}_{14} \mathrm{H}_{15} \mathrm{NO}_{3}\left(\mathrm{M}^{+}\right): 245.1052$, found: 245.1064 .

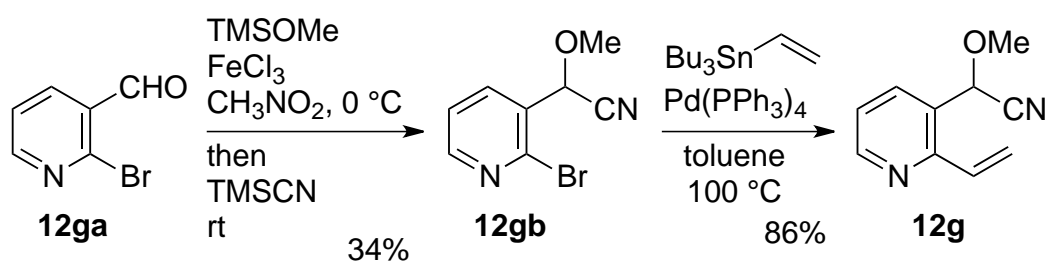

2-(2-Bromopyridin-3-yl)-2-methoxyacetonitrile (12gb): A mixture of methoxytrimethylsilane 
(1.82 mL, $13.2 \mathrm{mmol}), \mathrm{FeCl}_{3}(17.8 \mathrm{mg}, 0.11 \mathrm{mmol})$, and 2-bromonicotinaldehyde (12ga) (1.02 g, $5.5 \mathrm{mmol})$ in nitromethane $(2.8 \mathrm{~mL})$ was stirred at $0{ }^{\circ} \mathrm{C}$ for $3 \mathrm{~h}$. Trimethylsilyl cyanide $(1.82 \mathrm{~mL}$, $13.2 \mathrm{mmol}$ ) was then added and the mixture was stirred at room temperature for $24 \mathrm{~h}$. Saturated aqueous sodium bicarbonate (ca. $3 \mathrm{~mL}$ ) was added to the mixture and the products were extracted with $\mathrm{Et}_{2} \mathrm{O}$. The combined organic layers were dried over $\mathrm{MgSO}_{4}$ and concentrated under reduced pressure. Purification by flash column chromatography $\left(\mathrm{SiO}_{2}\right.$, hexane- $\left.\mathrm{Et}_{2} \mathrm{O}=3: 1\right)$ afforded cyanohydrin methyl ether $\mathbf{1 2 g b}$ (424.6 $\mathrm{mg}, 1.87 \mathrm{mmol}, 34 \%)$.

Yellow solid; M.p. $55-57^{\circ} \mathrm{C} ;{ }^{1} \mathrm{H}$ NMR $\left(500 \mathrm{MHz}, \mathrm{CDCl}_{3}\right) \delta 8.44(1 \mathrm{H}, \mathrm{d}, J=3.5 \mathrm{~Hz}), 7.99(1 \mathrm{H}, \mathrm{d}$, $J=7.5 \mathrm{~Hz}), 7.40(1 \mathrm{H}, \mathrm{dd}, J=7.5,4.6 \mathrm{~Hz}), 5.36(1 \mathrm{H}, \mathrm{s}), 3.67(3 \mathrm{H}, \mathrm{s}) ;{ }^{13} \mathrm{C} \mathrm{NMR}\left(125 \mathrm{MHz}, \mathrm{CDCl}_{3}\right)$ $\delta 150.96,141.97,137.18,130.67,123.27,115.52$, 70.82, 58.44; IR (ATR) v 3006, 2936, 2832, 2158, 1562, 1406, 1301, 1051, 968, 804, $715 \mathrm{~cm}^{-1}$; HRMS (FD) calcd for $\mathrm{C}_{8} \mathrm{H}_{7} \mathrm{BrN}_{2} \mathrm{O}\left(\mathrm{M}^{+}\right)$: 225.9742, found: 225.9758 .

2-Methoxy-2-(2-vinylpyridin-3-yl)acetonitrile (12g): To a mixture of cyanohydrin methyl ether 12gb (424.6 mg, $1.87 \mathrm{mmol})$ and tributyl(vinyl)stannane $(783 \mu \mathrm{L}, 2.6 \mathrm{mmol})$ in toluene $(5.4 \mathrm{~mL})$ was added $\mathrm{Pd}\left(\mathrm{PPh}_{3}\right)_{4}(100.5 \mathrm{mg}, 0.087 \mathrm{mmol})$ and the mixture was stirred at $100{ }^{\circ} \mathrm{C}$ for $4 \mathrm{~h}$. The mixture was filtered through a pad of Celite and the filtrate was concentrated under reduced pressure. Purification by flash column chromatography $\left(10 \mathrm{wt} \% \mathrm{~K}_{2} \mathrm{CO}_{3} / \mathrm{SiO}_{2}\right.$, hexane-Et $\left.{ }_{2} \mathrm{O}=3: 1\right)$ afforded nitrile 12g (280.8 mg, $1.61 \mathrm{mmol}, 86 \%)$.

Green oil; ${ }^{1} \mathrm{H}$ NMR $\left(500 \mathrm{MHz}, \mathrm{CDCl}_{3}\right) 8.65(1 \mathrm{H}, \mathrm{dd}, J=5.2,1.8 \mathrm{~Hz}), 7.92(1 \mathrm{H}, \mathrm{dd}, J=8.0,1.7$ $\mathrm{Hz}), 7.28(1 \mathrm{H}, \mathrm{dd}, J=8.0,4.6 \mathrm{~Hz}), 6.97(1 \mathrm{H}, \mathrm{dd}, J=16.6,10.9 \mathrm{~Hz}), 6.46(1 \mathrm{H}, \mathrm{dd}, J=17.2,1.8$ $\mathrm{Hz}), 5.67(1 \mathrm{H}, \mathrm{dd}, J=10.9,1.8 \mathrm{~Hz}), 5.40(1 \mathrm{H}, \mathrm{s}), 3.58(3 \mathrm{H}, \mathrm{s}) ;{ }^{13} \mathrm{C} \mathrm{NMR}\left(125 \mathrm{MHz}, \mathrm{CDCl}_{3}\right) \delta$ 153.43, 150.55, 136.03, 131.22, 125.73, 122.60, 122.54, 116.04, 69.46, 57.50; IR (ATR) v 2932, 2831, 2191, 1561, 1311, 1188, 1065, 970, 926, $798 \mathrm{~cm}^{-1}$; HRMS (FD) calcd for $\mathrm{C}_{10} \mathrm{H}_{10} \mathrm{~N}_{2} \mathrm{O}\left(\mathrm{M}^{+}\right)$: 174.0793, found: 174.0801 .

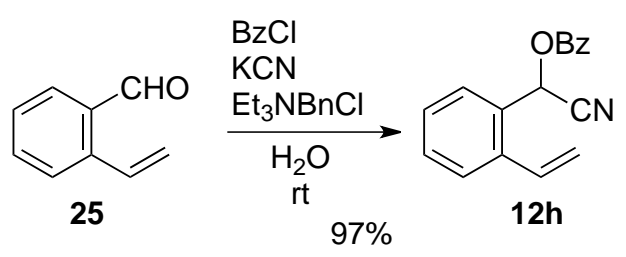

Cyano(2-vinylphenyl)methyl benzoate (12h): To a mixture of $o$-vinylbenzaldehyde $(\mathbf{2 5})^{2}(660.8$ $\mathrm{mg}, 5.0 \mathrm{mmol})$, benzoyl chloride $(749 \mu \mathrm{L}, 6.5 \mathrm{mmol})$, and $\mathrm{KCN}(488.4 \mathrm{mg}, 7.5 \mathrm{mmol})$ in $\mathrm{H}_{2} \mathrm{O}(1.5$ $\mathrm{mL}$ ) was added benzyltriethylammonium chloride $(34.0 \mathrm{mg}, 0.15 \mathrm{mmol}$ ) and the reaction mixture was stirred at room temperature for $26 \mathrm{~h}$. Saturated aqueous sodium bicarbonate (ca. $10 \mathrm{~mL}$ ) was added to the mixture and the products were extracted with $\mathrm{Et}_{2} \mathrm{O}$. The combined organic layers were dried over $\mathrm{MgSO}_{4}$ and concentrated under reduced pressure. Purification by flash column chromatography $\left(\mathrm{SiO}_{2}\right.$, hexane-EtOAc $\left.=8: 1\right)$ afforded $O$-benzoyl cyanohydrin $\mathbf{1 2 h}(1.28 \mathrm{~g}, 4.86$ mmol, 97\%). 
Yellow oil; ${ }^{1} \mathrm{H}$ NMR $\left(500 \mathrm{MHz}, \mathrm{CDCl}_{3}\right) \delta 8.06(2 \mathrm{H}, \mathrm{d}, J=7.5 \mathrm{~Hz}), 7.71(1 \mathrm{H}, \mathrm{dd}, J=7.4,1.2 \mathrm{~Hz})$, $7.62(1 \mathrm{H}, \mathrm{t}, J=7.5 \mathrm{~Hz}), 7.58(1 \mathrm{H}, \mathrm{d}, J=7.4 \mathrm{~Hz}), 7.49-7.44(3 \mathrm{H}, \mathrm{m}), 7.41(1 \mathrm{H}, \mathrm{td}, J=7.5,1.2 \mathrm{~Hz})$, $7.07(1 \mathrm{H}, \mathrm{dd}, J=17.2,10.9 \mathrm{~Hz}), 6.82(1 \mathrm{H}, \mathrm{s}), 5.73(1 \mathrm{H}, \mathrm{d}, J=17.2 \mathrm{~Hz}), 5.50(1 \mathrm{H}, \mathrm{dd}, J=10.9$, $1.2 \mathrm{~Hz}) ;{ }^{13} \mathrm{C}$ NMR $\left(125 \mathrm{MHz}, \mathrm{CDCl}_{3}\right) \delta 164.42,137.53,134.04,132.44,130.68,129.99,128.76$, 128.58, 128.53, 128.36, 127.94, 127.24, 119.26, 115.99, 61.32; IR (ATR) v 3066, 2955, 2361, 1728, 1601, 1452, 1240, 1065, 992, 939, 771, $706 \mathrm{~cm}^{-1}$; HRMS (FD) calcd for $\mathrm{C}_{17} \mathrm{H}_{13} \mathrm{NO}_{2}\left(\mathrm{M}^{+}\right)$: 263.0946, found: 263.0949 .
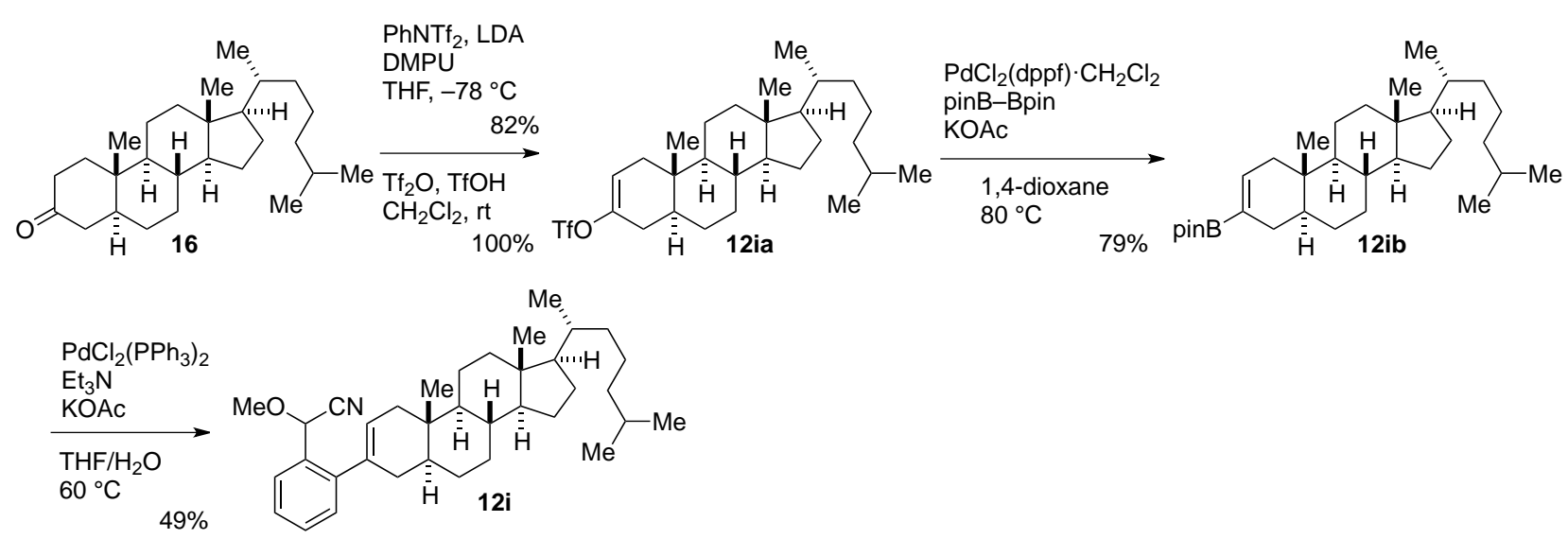

$(5 S, 8 R, 9 S, 10 S, 13 R, 14 S, 17 R)-10,13-D i m e t h y l-17-(6-m e t h y l h e p t a n-2-y l)-4,5,6,7,8,9,10,11,12,13$, 14,15,16,17-tetradecahydro-1H-cyclopenta[a]phenanthren-3-yl trifluoromethanesulfonate (12ia): Vinyl triflate 12ia was prepared according to the reported procedure. ${ }^{3,4}$ To a solution of $i$ - $\operatorname{Pr}_{2} \mathrm{NH}(727 \mu \mathrm{L}, 5.16 \mathrm{mmol})$ in THF $(10 \mathrm{~mL})$ was added $n$-BuLi $(2.65 \mathrm{M}$ in hexane, $1.85 \mathrm{~mL}, 4.9$ $\mathrm{mmol}$ ) at $0{ }^{\circ} \mathrm{C}$ and the mixture was stirred at this temperature for $30 \mathrm{~min}$. After cooled to $-78{ }^{\circ} \mathrm{C}$, a solution of ketone 16 (1.73g $4.45 \mathrm{mmol})$ and DMPU (1.61 mL, $13.4 \mathrm{mmol})$ in THF (8 mL) was added to the resulting LDA solution and the reaction mixture was stirred at $-78{ }^{\circ} \mathrm{C}$ for $2 \mathrm{~h}$. A solution of $\mathrm{PhNTf}_{2}(1.84 \mathrm{~g}, 5.16 \mathrm{mmol})$ in THF $(8 \mathrm{~mL})$ was then added to the reaction mixture and the mixture was stirred at $-78{ }^{\circ} \mathrm{C}$ for $1.5 \mathrm{~h}$. To the mixture was added saturated aqueous $\mathrm{NH}_{4} \mathrm{Cl}$ $(10 \mathrm{~mL})$ and the products were extracted with hexane. The combined organic layers were died over $\mathrm{MgSO}_{4}$ and concentrated under reduced pressure. Purification by flash column chromatography $\left(\mathrm{SiO}_{2}\right.$, hexane only) afforded vinyl triflate 12ia $(1.90 \mathrm{~g}, 3.66 \mathrm{mmol}, 82 \%)$ as a 71:29 mixture of diastereomers.

To a solution of the above 71:29 diastereomeric mixture of vinyl triflate 12ia (1.85 g, $3.57 \mathrm{mmol})$ and $\mathrm{Tf}_{2} \mathrm{O}(0.36 \mathrm{~mL}, 2.19 \mathrm{mmol})$ in $\mathrm{CH}_{2} \mathrm{Cl}_{2}(18 \mathrm{~mL})$ was added $\mathrm{TfOH}(100 \mu \mathrm{L}, 1.13 \mathrm{mmol})$ and reaction mixture was stirred at room temperature for $24 \mathrm{~h}$. Saturated sodium bicarbonate $(10 \mathrm{~mL})$ was added to the mixture and the products were extracted with hexane. The combined organic layers were dried over $\mathrm{MgSO}_{4}$ and concentrated under reduced pressure to give the isomerized vinyl triflate 12ia $(1.85 \mathrm{~g}, 3.57 \mathrm{mmol}, 100 \%)$ as a 89:11 mixture of diastereomers.

White solid; M.p. $87-90^{\circ} \mathrm{C}$; $[\alpha]_{\mathrm{D}}{ }^{23} 5.95$ (c 3.09, $\left.\mathrm{CHCl}_{3}\right) ;{ }^{1} \mathrm{H}$ NMR of major isomer: $(500 \mathrm{MHz}$, $\left.\mathrm{CDCl}_{3}\right) \delta 5.67-5.62(1 \mathrm{H}, \mathrm{m}), 2.19-2.02(3 \mathrm{H}, \mathrm{m}), 2.02-1.95(1 \mathrm{H}, \mathrm{m}), 1.92-1.77(2 \mathrm{H}, \mathrm{m})$, 
$1.72-1.65(1 \mathrm{H}, \mathrm{m}), 1.62-1.19(14 \mathrm{H}, \mathrm{m}), 1.19-0.93(8 \mathrm{H}, \mathrm{m}), 0.90(3 \mathrm{H}, \mathrm{d}, J=6.3 \mathrm{~Hz}), 0.865(3 \mathrm{H}, \mathrm{d}$, $J=6.3 \mathrm{~Hz}), 0.860(3 \mathrm{H}, \mathrm{d}, J=6.3 \mathrm{~Hz}), 0.79(3 \mathrm{H}, \mathrm{s}), 0.66(3 \mathrm{H}, \mathrm{s}) ;{ }^{13} \mathrm{C}$ NMR of major isomer: (125 $\left.\mathrm{MHz}, \mathrm{C}_{6} \mathrm{D}_{6}\right) \delta 147.82,119.22$ [q, ${ }^{1} J(\mathrm{C}, \mathrm{F})=318.4 \mathrm{~Hz}$ ], 117.33, 56.66, 56.45, 53.36, 42.69, 42.06, $40.18,39.92$, 38.36, 36.63, 36.19, 35.50, 34.32, 32.20, 31.62, 28.56, 28.41, 28.19, 24.45, 24.36, 23.02, 22.76, 21.40, 18.97, 12.17, 11.44; IR (ATR) v 2927, 1415, 1247, 1219, 1200, 1141, 1038, 872, 772, 613, $454 \mathrm{~cm}^{-1}$; HRMS (FD) calcd for $\mathrm{C}_{28} \mathrm{H}_{45} \mathrm{~F}_{3} \mathrm{O}_{3} \mathrm{~S}\left(\mathrm{M}^{+}\right)$: 518.3042, found: 518.3054 .

2-((5S,8R,9S,10S,13R,14S,17R)-10,13-dimethyl-17-(6-methylheptan-2-yl)-4,5,6,7,8,9,10,11,12, 13,14,15,16,17-tetradecahydro-1H-cyclopenta[a]phenanthren-3-yl)-4,4,5,5-tetramethyl-1,3,2dioxaborolane (12ib): Boronate ester 12ib was prepared according to the reported procedure. ${ }^{5}$ To a solution of the above vinyl triflate $12 \mathrm{ia}(1.55 \mathrm{~g}, 3.0 \mathrm{mmol})$, KOAc $(883.4 \mathrm{mg}, 9.0 \mathrm{mmol})$, and bis(pinacolato)diboron $(761.8 \mathrm{mg}, \quad 3.0 \mathrm{mmol})$ in 1,4-dioxane $(15 \mathrm{~mL})$ was added $\mathrm{PdCl}_{2}$ (dppf) $\mathrm{CH}_{2} \mathrm{Cl}_{2}(122.5 \mathrm{mg}, 0.15 \mathrm{mmol})$ and the reaction mixture was stirred at $80{ }^{\circ} \mathrm{C}$ for $3 \mathrm{~h}$. The mixture was filtered through a pad of Celite and the filtrate was concentrated under reduced pressure. Purification by flash column chromatography $\left(\mathrm{SiO}_{2}\right.$, hexane-EtOAc $\left.=10: 1\right)$ afforded boronate ester 12ib (1.17 g, $2.36 \mathrm{mmol}, 79 \%)$ as a 91:9 mixture of diastereomers.

White solid; M.p. $177-179{ }^{\circ} \mathrm{C}$; $[\alpha]_{\mathrm{D}}{ }^{23} 58.8$ (c 1.09, $\left.\mathrm{CHCl}_{3}\right) ;{ }^{1} \mathrm{H}$ NMR $\left(500 \mathrm{MHz}, \mathrm{CDCl}_{3}\right) \delta 6.48$ $(1 \mathrm{H}, \mathrm{d}, J=4.0 \mathrm{~Hz}), 2.09-1.93(3 \mathrm{H}, \mathrm{m}), 1.86-0.93(38 \mathrm{H}, \mathrm{m}), 0.90(3 \mathrm{H}, \mathrm{d}, J=6.3 \mathrm{~Hz}), 0.860(3 \mathrm{H}$, $\mathrm{d}, J=6.3 \mathrm{~Hz}), 0.856(3 \mathrm{H}, \mathrm{d}, J=6.3 \mathrm{~Hz}), 0.71(3 \mathrm{H}, \mathrm{s}), 0.65(3 \mathrm{H}, \mathrm{s}) ;{ }^{13} \mathrm{C} \mathrm{NMR}\left(125 \mathrm{MHz}, \mathrm{C}_{6} \mathrm{D}_{6}\right) \delta$ $142.37,82.91,56.67,56.63,54.19,42.74,41.82$, 41.78, 40.32, 39.90, 36.65, 36.23, 35.83, 34.51, $32.14,29.09,28.62,28.39,25.03,24.49,24.37,23.04,22.78,21.26,19.02,12.24,12.17$; IR $\left(\right.$ ATR) $v 2927,2339,2165,1974,1635,1390,1337,1310 \mathrm{~cm}^{-1}$; HRMS (FD) calcd for $\mathrm{C}_{33} \mathrm{H}_{57} \mathrm{BO}_{2}$ $\left(\mathrm{M}^{+}\right)$: 496.4452, found: 496.4458 .

\section{2-(2-((5S,8R,9S,10S,13R,14S,17R)-10,13-dimethyl-17-(6-methylheptan-2-yl)-4,5,6,7,8,9,10,11,}

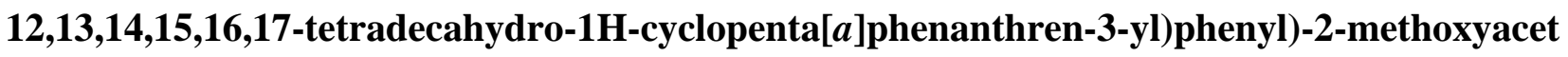
onitrile (12i): To a mixture of boronate ester 12ib (565.0 mg, $1.14 \mathrm{mmol})$, KOAc (167.8 mg, 1.71 $\mathrm{mmol})$, and $\mathrm{PdCl}_{2}\left(\mathrm{PPh}_{3}\right)_{2}(35.1 \mathrm{mg}, 0.0500 \mathrm{mmol})$ in $\mathrm{THF}-\mathrm{H}_{2} \mathrm{O}(6: 1,3.8 \mathrm{~mL})$ were added $\mathrm{Et}_{3} \mathrm{~N}$ $(120 \mu \mathrm{L}, 0.86 \mathrm{mmol})$ and 2-(2-bromophenyl)-2-methoxyacetonitrile $(353 \mu \mathrm{L}, 2.28 \mathrm{mmol})$ and the reaction mixture was stirred at $60{ }^{\circ} \mathrm{C}$ for $12 \mathrm{~h}$. The mixture was filtered through a pad of Celite and the filtrate was concentrated under reduced pressure. Purification by flash column chromatography $\left(\mathrm{SiO}_{2}\right.$, hexane- $\left.\mathrm{Et}_{2} \mathrm{O}=30: 1\right)$ afforded nitrile $12 \mathbf{i}(291.0 \mathrm{mg}, 0.564 \mathrm{mmol}, 49 \%)$ as a 50:50 mixture of diastereomers.

White solid; M.p. $106-118{ }^{\circ} \mathrm{C} ;[\alpha]_{\mathrm{D}}{ }^{23} 52.8$ (c $\left.0.570, \mathrm{CHCl}_{3}\right) ;{ }^{1} \mathrm{H}$ NMR $\left(500 \mathrm{MHz}, \mathrm{CDCl}_{3}\right) \delta$ 7.68-7.62 (1H, m), 7.38-7.29 (2H, m), $7.44(1 \mathrm{H}, \mathrm{d}, J=7.5 \mathrm{~Hz}), 5.57(1 \mathrm{H}, \mathrm{s}), 5.30(0.5 \mathrm{H}, \mathrm{s}), 5.24$ $(0.5 \mathrm{H}, \mathrm{s}), 3.51(1.5 \mathrm{H}, \mathrm{s}), 3.50(1.5 \mathrm{H}, \mathrm{s}), 2.22-1.76(6 \mathrm{H}, \mathrm{m}), 1.75-0.96(22 \mathrm{H}, \mathrm{m}), 0.92(3 \mathrm{H}, \mathrm{d}, J=$ $5.0 \mathrm{~Hz}), 0.89-0.83(9 \mathrm{H}, \mathrm{m}), 0.81-0.72(1 \mathrm{H}, \mathrm{m}), 0.69(3 \mathrm{H}, \mathrm{s}) ;{ }^{13} \mathrm{C} \mathrm{NMR}\left(125 \mathrm{MHz}, \mathrm{CDCl}_{3}\right) \delta$ $143.93,143.88,134.96,134.95,130.77,130.68,129.55$, 129.52, 128.74, 128.62, 128.02, 127.95, 
$127.27,127.19,117.87,69.97,69.78,57.57,57.44,56.44,56.25,53.85,42.48,41.94,41.89,40.23$, 40.17, 39.97, 39.50, 36.17, 35.78, 35.77, 35.71, 35.63, 35.61, 34.13, 34.10, 31.67, 28.51, 28.48, $28.21,28.00,24.20,23.82,22.81,22.56,21.09,18.69,12.20,12.09,12.01$; IR (ATR) v 3734, 2929, 2868, 2360, 2336, 1458, 1445, 1382, 1083, $757 \mathrm{~cm}^{-1}$; HRMS (FD) calcd for $\mathrm{C}_{36} \mathrm{H}_{53} \mathrm{NO}$ $\left(\mathrm{M}^{+}\right)$: 515.4127, found: 515.4130.

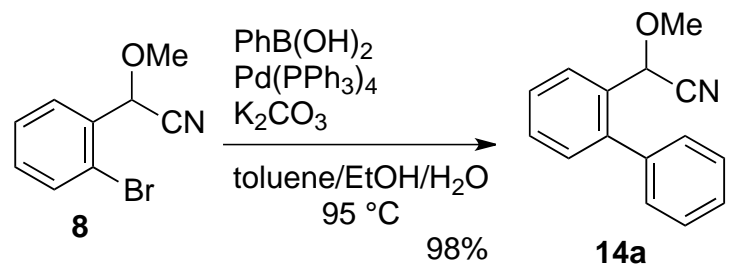

2-([1,1'-Biphenyl]-2-yl)-2-methoxyacetonitrile (14a): To a mixture of cyanohydrin methyl ether 8 (1.21 g, $1.08 \mathrm{~mL}, 7.0 \mathrm{mmol}$ ), phenyl boronic acid (938.9 mg, $7.7 \mathrm{mmol})$, and $\mathrm{K}_{2} \mathrm{CO}_{3}(1.93 \mathrm{~g}$, $14.0 \mathrm{mmol})$ in toluene- $\mathrm{EtOH}-\mathrm{H}_{2} \mathrm{O}(3: 3: 1,23 \mathrm{~mL})$ was added $\mathrm{Pd}\left(\mathrm{PPh}_{3}\right)_{4}(242.7 \mathrm{mg}, 0.21 \mathrm{mmol})$ and the reaction mixture was stirred at $95{ }^{\circ} \mathrm{C}$ for $4 \mathrm{~h}$. Water $(15 \mathrm{~mL})$ was added to the mixture and the products were extracted with EtOAc. The combined organic layers were dried over $\mathrm{MgSO}_{4}$ and concentrated under reduced pressure. Purification by flash column chromatography $\left(\mathrm{SiO}_{2}\right.$, hexane- $\left.\mathrm{Et}_{2} \mathrm{O}=10: 1\right)$ afforded nitrile $14 \mathrm{a}(1.54 \mathrm{~g}, 6.88 \mathrm{mmol}, 98 \%)$.

White solid; M.p. $43-46{ }^{\circ} \mathrm{C} ;{ }^{1} \mathrm{H}$ NMR $\left(500 \mathrm{MHz}, \mathrm{CDCl}_{3}\right) \delta 7.82-7.76(1 \mathrm{H}, \mathrm{m}), 7.52-7.40$ (5H, m), 7.38-7.31 (3H, m), $5.00(1 \mathrm{H}, \mathrm{s}), 3.44(3 \mathrm{H}, \mathrm{s}) ;{ }^{13} \mathrm{C} \mathrm{NMR}\left(125 \mathrm{MHz}, \mathrm{CDCl}_{3}\right) \delta$ 141.96, 139.23, $131.27,130.38,129.80,129.13,128.48,128.27,128.21,127.93,117.55,69.87,57.38$; IR (ATR) $v$ 3057, 2927, 2824, 2337, 1478, 1186, 1073, 930, 762, 701, $507 \mathrm{~cm}^{-1}$; HRMS (FD) calcd for $\mathrm{C}_{15} \mathrm{H}_{13} \mathrm{NO}\left(\mathrm{M}^{+}\right)$: 223.0997, found: 223.1005.

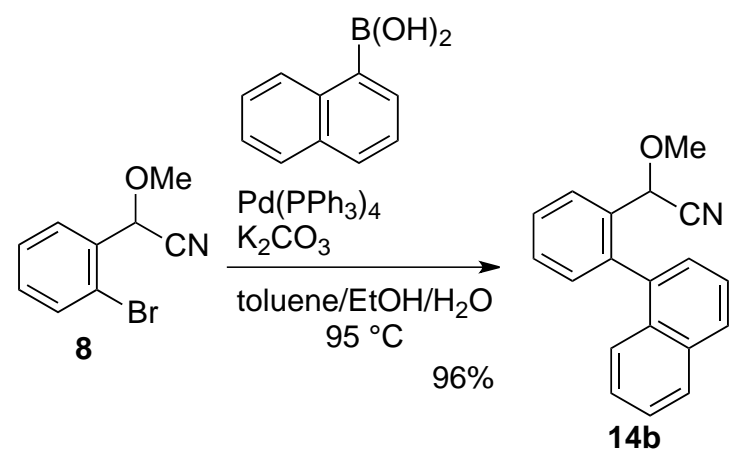

2-Methoxy-2-(2-(naphthalen-1-yl)phenyl)acetonitrile (14b): To a mixture of cyanohydrin methyl ether 8 (346 mg, $310 \mu \mathrm{L}, 2.0 \mathrm{mmol}$ ), 1-naphthalene boronic acid (378.4 mg, $2.2 \mathrm{mmol}$ ), and $\mathrm{K}_{2} \mathrm{CO}_{3}(552.8 \mathrm{mg}, 4.0 \mathrm{mmol})$ in toluene-EtOH- $\mathrm{H}_{2} \mathrm{O}(3: 3: 1,6.7 \mathrm{~mL})$ was added $\mathrm{Pd}\left(\mathrm{PPh}_{3}\right)_{4}$ $(69.3 \mathrm{mg}, 0.060 \mathrm{mmol})$ and the reaction mixture was stirred at $95{ }^{\circ} \mathrm{C}$ for $5 \mathrm{~h}$. Brine $(5 \mathrm{~mL})$ was added to the mixture and the products were extracted with EtOAc. The combined organic layers were dried over $\mathrm{MgSO}_{4}$ and concentrated under reduced pressure. Purification by flash column 
chromatography $\left(\mathrm{SiO}_{2}\right.$, hexane- $\left.\mathrm{Et}_{2} \mathrm{O}=10: 1\right)$ afforded nitrile $\mathbf{1 4 b}(524.1 \mathrm{mg}, 1.92 \mathrm{mmol}, 96 \%)$ as a 50:50 mixture of atropisomers.

Colorless oil; ${ }^{1} \mathrm{H}$ NMR (500 MHz, $\left.\mathrm{CDCl}_{3}\right) \delta$ 7.96-7.91 (2H, m), $7.87(0.5 \mathrm{H}, \mathrm{d}, J=7.5 \mathrm{~Hz}), 7.83$ $(0.5 \mathrm{H}, \mathrm{d}, J=8.0 \mathrm{~Hz}), 7.61-7.48(4 \mathrm{H}, \mathrm{m}), 7.47-7.33(4 \mathrm{H}, \mathrm{m}), 4.68(0.5 \mathrm{H}, \mathrm{s}), 4.66(0.5 \mathrm{H}, \mathrm{s}), 3.25$ $(1.5 \mathrm{H}, \mathrm{s}), 3.10(1.5 \mathrm{H}, \mathrm{s}) ;{ }^{13} \mathrm{C} \mathrm{NMR}\left(125 \mathrm{MHz}, \mathrm{CDCl}_{3}\right) \delta 140.01,139.44,136.40,136.22,133.50$, $133.43,132.95,132.60,132.04,131.88,131.15,131.02$, 129.79, 129.52, 128.66, 128.53, 128.33, $128.25,127.17,127.52,127.45,127.43,126.83,126.29,126.24,126.14,125.73,125.48,125.29$, $125.00,117.53,117.44,69.87,69.63,57,75,57.44$; IR (ATR) v 3058, 2928, 2826, 2027, 1448, 1394, 1329, 1271, 1192, 1081, 967, $779 \mathrm{~cm}^{-1}$; HRMS (FD) calcd for $\mathrm{C}_{19} \mathrm{H}_{15} \mathrm{NO}\left(\mathrm{M}^{+}\right)$: 273.1154, found: 273.1160 .

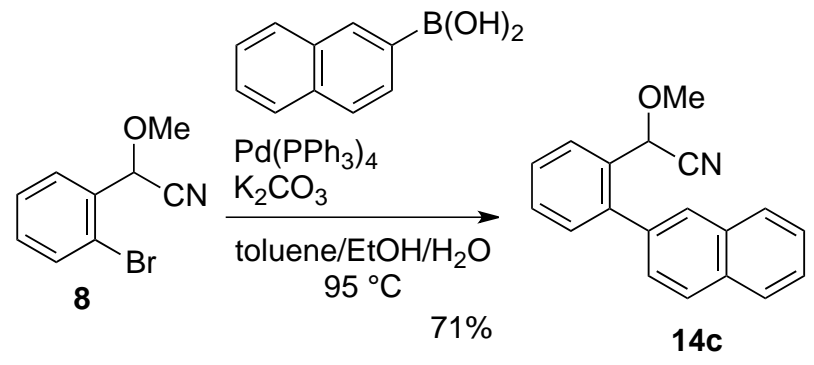

2-Methoxy-2-(2-(naphthalen-2-yl)phenyl)acetonitrile (14c): To a mixture of cyanohydrin methyl ether $8(310 \mu \mathrm{L}, 2.0 \mathrm{mmol})$, 2-naphthalene boronic acid (378.4 mg, $2.2 \mathrm{mmol})$, and $\mathrm{K}_{2} \mathrm{CO}_{3}$ $(552.8 \mathrm{mg}, 4.00 \mathrm{mmol})$ in toluene-EtOH$-\mathrm{H}_{2} \mathrm{O}(3: 3: 1,6.7 \mathrm{~mL})$ was added $\mathrm{Pd}\left(\mathrm{PPh}_{3}\right)_{4}(69.3 \mathrm{mg}$, $0.0600 \mathrm{mmol})$ and the reaction mixture was stirred at $95{ }^{\circ} \mathrm{C}$ for $6 \mathrm{~h}$. Brine $(5 \mathrm{~mL})$ was added to the mixture and the products were extracted with EtOAc. The combined organic layers were dried over $\mathrm{MgSO}_{4}$ and concentrated under reduced pressure. Purification by flash column chromatography $\left(\mathrm{SiO}_{2}\right.$, hexane- $\left.\mathrm{Et}_{2} \mathrm{O}=20: 1\right)$ afforded nitrile $14 \mathrm{c}(388.8 \mathrm{mg}, 1.42 \mathrm{mmol}, 71 \%)$.

White solid; M.p. 43-45 ${ }^{\circ} \mathrm{C} ;{ }^{1} \mathrm{H}$ NMR (500 MHz, $\left.\mathrm{CDCl}_{3}\right) \delta$ 7.93-7.86 (3H, m), 7.83 (2H, s), 7.60-7.50 (4H, m), $7.49(1 \mathrm{H}, \mathrm{d}, J=8.0 \mathrm{~Hz}), 7.46-7.41(1 \mathrm{H}, \mathrm{m}), 5.04(1 \mathrm{H}, \mathrm{s}), 3.45(3 \mathrm{H}, \mathrm{s}) ;{ }^{13} \mathrm{C}$ NMR $\left(125 \mathrm{MHz}, \mathrm{CDCl}_{3}\right) \delta 141.88,136.60,133.03,132.63,131.50,130.57,129.83,128.35$, 128.29, 128.20, 128.18, 128.06, 127.72, 127.05, 126.70, 126.51, 117.56, 69.96, 57.47; IR (ATR) v 3053, 2931, 2829, 2340, 1448, 1327, 1185, 1072, 957, 763, $478 \mathrm{~cm}^{-1}$; HRMS (FD) calcd for $\mathrm{C}_{19} \mathrm{H}_{15} \mathrm{NO}\left(\mathrm{M}^{+}\right): 273.1154$, found: 273.1147. 


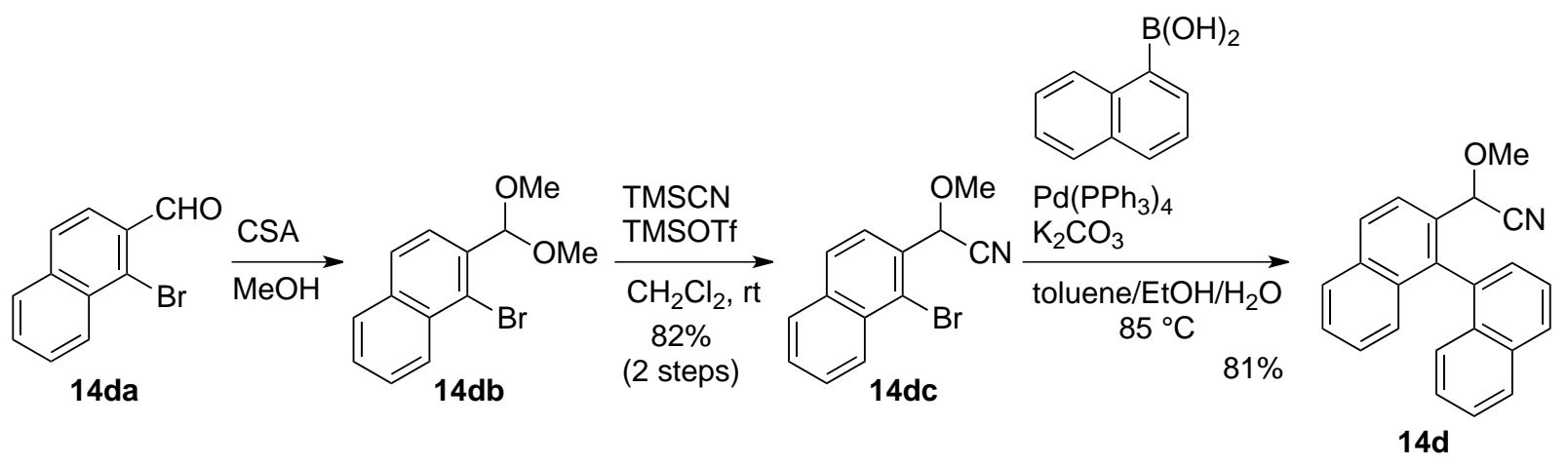

2-(1-Bromonaphthalen-2-yl)-2-methoxyacetonitrile (14dc): To a solution of 1-bromo-2-naphthaldehyde (14da) (278.0 mg, $1.18 \mathrm{mmol})$ in $\mathrm{MeOH}$ (4 mL) was added (+)-10-camphorsulfonic acid $(27.4 \mathrm{mg}, 0.118 \mathrm{mmol})$ and the reaction mixture was stirred at room temperature for $3 \mathrm{~h}$. Saturated aqueous sodium bicarbonate $(2 \mathrm{~mL})$ was added to the mixture and the products were extracted with $\mathrm{Et}_{2} \mathrm{O}$. The combined organic layers were dried over $\mathrm{MgSO}_{4}$ and concentrated under reduced pressure. The crude dimethyl acetal 14db (335.2 $\mathrm{mg})$ was used for the next step without further purification.

To a mixture of the above crude dimethyl acetal $\mathbf{1 4 d b}(335.2 \mathrm{mg}$, as $1.18 \mathrm{mmol})$ and TMSCN $(221 \mu \mathrm{L}, 1.77 \mathrm{mmol})$ in $\mathrm{CH}_{2} \mathrm{Cl}_{2}(6 \mathrm{~mL})$ was added TMSOTf $(21 \mu \mathrm{L}, 0.118 \mathrm{mmol})$ and the reaction mixture was stirred at room temperature for $18 \mathrm{~h}$. Saturated aqueous sodium bicarbonate $(2 \mathrm{~mL})$ was added to the mixture and the products were extracted with $\mathrm{Et}_{2} \mathrm{O}$. The combined organic layers were dried over $\mathrm{MgSO}_{4}$ and concentrated under reduced pressure. Purification by flash column chromatography $\left(\mathrm{SiO}_{2}\right.$, hexane-EtOAc $\left.=4: 1\right)$ afforded cyanohydrin methyl ether 14dc $(268.1 \mathrm{mg}$, $0.971 \mathrm{mmol}, 82 \%$ for 2 steps).

Brown solid; M.p. $55-57{ }^{\circ} \mathrm{C} ;{ }^{1} \mathrm{H}$ NMR $\left(500 \mathrm{MHz}, \mathrm{CDCl}_{3}\right) \delta 8.33(1 \mathrm{H}, \mathrm{d}, J=8.6 \mathrm{~Hz}), 7.90(1 \mathrm{H}, \mathrm{d}$, $J=8.6 \mathrm{~Hz}), 7.86(1 \mathrm{H}, \mathrm{d}, J=8.1 \mathrm{~Hz}), 7.76(1 \mathrm{H}, \mathrm{d}, J=8.6 \mathrm{~Hz}), 7.65(1 \mathrm{H}, \mathrm{t}, J=6.9 \mathrm{~Hz}), 7.61-7.57$ $(1 \mathrm{H}, \mathrm{m}), 5.77(1 \mathrm{H}, \mathrm{s}), 3.67(3 \mathrm{H}, \mathrm{s}) ;{ }^{13} \mathrm{C} \mathrm{NMR}\left(125 \mathrm{MHz}, \mathrm{CDCl}_{3}\right) \delta 134.44,131.64,130.59,128.47$, 128.03, 127.88, 127.52, 127.27, 124.21, 123.61, 116.48, 72.26, 57.92; IR (ATR) $v 2929,2832$, 2336, 1503, 1458, 1326, 1104, 967, 815, 764, $660 \mathrm{~cm}^{-1}$; HRMS (FD) calcd for $\mathrm{C}_{13} \mathrm{H}_{10} \mathrm{BrNO}\left(\mathrm{M}^{+}\right)$: 274.9946, found: 274.9927.

2-([1,1'-Binaphthalen]-2-yl)-2-methoxyacetonitrile (14d): To a mixture of cyanohydrin methyl ether 14dc $(263.2 \mathrm{mg}, 0.953 \mathrm{mmol})$, 1-naphthalene boronic acid (180.6 mg, $1.05 \mathrm{mmol})$, and $\mathrm{K}_{2} \mathrm{CO}_{3}(264.0 \mathrm{mg}, 1.91 \mathrm{mmol})$ in toluene-EtOH$-\mathrm{H}_{2} \mathrm{O}(3: 3: 1,3.2 \mathrm{~mL})$ was added $\mathrm{Pd}\left(\mathrm{PPh}_{3}\right)_{4}(33.5$ $\mathrm{mg}, 0.0290 \mathrm{mmol})$ and the reaction mixture was stirred at $85{ }^{\circ} \mathrm{C}$ for $5 \mathrm{~h}$. Brine $(5 \mathrm{~mL})$ was added to the mixture and the products were extracted with $\mathrm{Et}_{2} \mathrm{O}$. The combined organic layers were dried over $\mathrm{MgSO}_{4}$ and concentrated under reduced pressure. Purification by flash column chromatography $\left(\mathrm{SiO}_{2}\right.$, hexane-EtOAc $\left.=10: 1\right)$ afforded nitrile 14d $(248.4 \mathrm{mg}, 0.768 \mathrm{mmol}, 81 \%)$ as a 63:37 mixture of atropisomers.

Colorless oil; ${ }^{1} \mathrm{H}$ NMR (500 MHz, $\left.\mathrm{CDCl}_{3}\right) \delta 8.08(1 \mathrm{H}, \mathrm{d}, J=8.6 \mathrm{~Hz}), 8.03(1 \mathrm{H}, \mathrm{d}, J=8.6 \mathrm{~Hz})$, 
8.01-7.88 (3H, m), 7.69-7.60 (1H, m), 7.56-7.41 (3H, m), 7.37-7.26 (2H, m), $7.18(2 \mathrm{H}, \mathrm{t}, J=7.5$ $\mathrm{Hz}), 4.70(1 \mathrm{H}, \mathrm{s}), 3.30(2 \mathrm{H}, \mathrm{s}), 3.12(1 \mathrm{H}, \mathrm{s}) ;{ }^{13} \mathrm{C} \mathrm{NMR}\left(125 \mathrm{MHz}, \mathrm{CDCl}_{3}\right) \delta 137.79,137.15$, 134.01, 133.97, 133.76, 133.67, 133.51, 133.42, 132.79, 132.67, 132.29, 130.35, 129.99, 129.25, $129.20,128.81,128.79,128.62,128.31,128.20,127.97,127.20,127.04,127.00,126.95,126.91$, $126.75,126.73,126.44,126.41,126.27,125.81,125.68,125.50,125.10,124.26,123.59,117.54$, 117.44, 70.00, 69.97, 57.72, 57.53; IR (ATR) v 3058, 2929, 2826, 1593, 1507, 1457, 1370, 1337, 1264, 1189, 1081, 957, 907, 782, $729 \mathrm{~cm}^{-1}$; HRMS (FD) calcd for $\mathrm{C}_{23} \mathrm{H}_{17} \mathrm{NO}\left(\mathrm{M}^{+}\right)$: 323.1310, found: 323.1317.

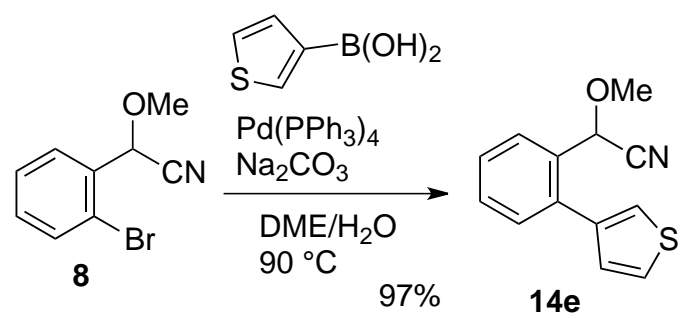

2-Methoxy-2-(2-(thiophen-3-yl)phenyl)acetonitrile (14e): To a mixture of cyanohydrin methyl ether 8 (173 mg, $155 \mu \mathrm{L}, 1.00 \mathrm{mmol})$, 3-thiophene boronic acid (160.0 mg, $1.25 \mathrm{mmol})$, and $\mathrm{Na}_{2} \mathrm{CO}_{3}$ (256.5 mg, $2.42 \mathrm{mmol}$ ) in DME- $\mathrm{H}_{2} \mathrm{O}(3: 2,3 \mathrm{~mL})$ was added $\mathrm{Pd}\left(\mathrm{PPh}_{3}\right)_{4}(57.8 \mathrm{mg}, 0.050$ $\mathrm{mmol})$ and the reaction mixture was stirred at $90{ }^{\circ} \mathrm{C}$ for $3 \mathrm{~h} . \mathrm{H}_{2} \mathrm{O}(2 \mathrm{~mL})$ was added to the mixture and the products were extracted with $\mathrm{Et}_{2} \mathrm{O}$. The combined organic layers were dried over $\mathrm{MgSO}_{4}$ and concentrated under reduced pressure. Purification by flash column chromatography $\left(\mathrm{SiO}_{2}\right.$, hexane-EtOAc = 8:1) afforded nitrile 14e $(223.1 \mathrm{mg}, 0.973 \mathrm{mmol}, 97 \%)$.

Yellow solid; M.p. $63-64{ }^{\circ} \mathrm{C} ;{ }^{1} \mathrm{H}$ NMR $\left(500 \mathrm{MHz}, \mathrm{CDCl}_{3}\right) \delta$ 7.81-7.76 (1H, m), 7.48-7.43 (3H, m), 7.42-7.36 (1H, m), 7.39-7.32 (1H, m), $7.16(1 \mathrm{H}, \mathrm{dd}, J=5.2,1.1 \mathrm{~Hz}), 5.12(1 \mathrm{H}, \mathrm{s}), 3.50(3 \mathrm{H}$, s); ${ }^{13} \mathrm{C}$ NMR $\left(125 \mathrm{MHz}, \mathrm{CDCl}_{3}\right) \delta 139.35,136.77,131.23,130.39,129.88,128.73,128.48,128.15$, 126.14, 123.86, 117.37, 70.00, 57.14; IR (ATR) v 3103, 2950, 2827, 2337, 1450, 1181, 1068, 969, $806,763 \mathrm{~cm}^{-1}$; HRMS (FD) calcd for $\mathrm{C}_{13} \mathrm{H}_{11} \mathrm{NOS}\left(\mathrm{M}^{+}\right)$: 229.0561, found: 229.0550 .

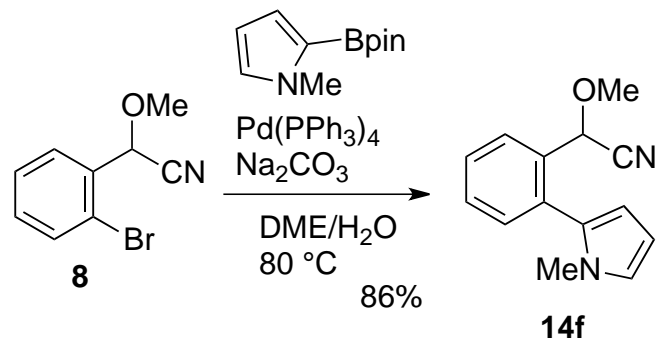

2-Methoxy-2-(2-(1-methyl-1H-pyrrol-2-yl)phenyl)acetonitrile (14f): To a mixture of cyanohydrin methyl ether $\mathbf{8}(866 \mathrm{mg}, 774 \mu \mathrm{L}, 5.00 \mathrm{mmol})$, 1-methyl-2-pyrroleboronic acid pinacol ester $(1.35 \mathrm{~g}, 6.5 \mathrm{mmol})$, and $\mathrm{Na}_{2} \mathrm{CO}_{3}(1.06 \mathrm{~g}, 10 \mathrm{mmol})$ in $\mathrm{DME}-\mathrm{H}_{2} \mathrm{O}(3: 2,47 \mathrm{~mL})$ was added $\mathrm{Pd}\left(\mathrm{PPh}_{3}\right)_{4}(346.7 \mathrm{mg}, 0.300 \mathrm{mmol})$ and the reaction mixture was stirred at $80{ }^{\circ} \mathrm{C}$ for $24 \mathrm{~h} . \mathrm{H}_{2} \mathrm{O}(2$ 
$\mathrm{mL}$ ) was added to the mixture and the products were extracted with EtOAc. The combined organic layers were dried over $\mathrm{MgSO}_{4}$ and concentrated under reduced pressure. Purification by flash column chromatography $\left(\mathrm{SiO}_{2}\right.$, hexane-EtOAc = 8:1) afforded nitrile $\mathbf{1 4 f}(972.1 \mathrm{mg}, 4.30 \mathrm{mmol}$, $86 \%)$.

Red oil; ${ }^{1} \mathrm{H}$ NMR $\left(500 \mathrm{MHz}, \mathrm{CDCl}_{3}\right) \delta$ 7.80-7.75 (1H, m), 7.52-7.44 (2H, m), 7.35-7.30 (1H, m), 7.68-7.64 (1H, m), 6.24-6.21 (1H, m), 6.17-6.14 (1H, m), $5.13(1 \mathrm{H}, \mathrm{s}), 3.45(3 \mathrm{H}, \mathrm{s}), 3.44(3 \mathrm{H}, \mathrm{s})$; ${ }^{13} \mathrm{C} \mathrm{NMR}\left(125 \mathrm{MHz}, \mathrm{CDCl}_{3}\right) \delta 133.86,132.70,131.44,129.39,129.34,128.67,127.95,123.13$, 110.08, 107.63, 69.90, 57.57, 34.31; IR (ATR) v 2934, 2826, 2356, 1734, 1480, 1309, 1242, 1188, 1078, 970, 927, 764, $714 \mathrm{~cm}^{-1}$; HRMS (FD) calcd for $\mathrm{C}_{14} \mathrm{H}_{14} \mathrm{~N}_{2} \mathrm{O}\left(\mathrm{M}^{+}\right)$: 226.1106, found: 226.1114.

Synthesis of Aryl Amines 10, 11, 13a-e, and 13g-i (Schemes 4 and 5)

Synthesis of Aryl Amine 10

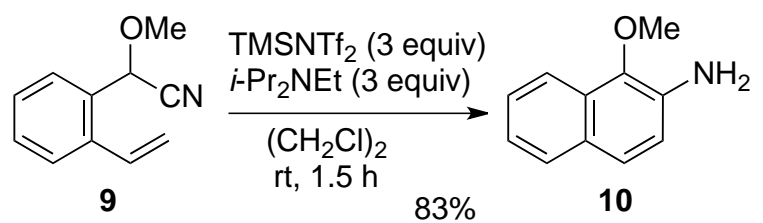

1-Methoxynaphthalen-2-amine (10): To a mixture of nitrile $9(52.0 \mathrm{mg}, 50 \mu \mathrm{L}, 0.300 \mathrm{mmol})$ and $\mathrm{Et}_{3} \mathrm{~N}(147 \mu \mathrm{L}, 0.900 \mathrm{mmol})$ was added $\mathrm{TMSNTf}_{2}(206 \mu \mathrm{L}, 0.900 \mathrm{mmol})$ and the reaction mixture was stirred at room temperature for $1.5 \mathrm{~h}$. Saturated aqueous sodium bicarbonate $(0.1 \mathrm{~mL})$ was added and the mixture was stirred at room temperature for $1 \mathrm{~h}$. After the mixture was extracted with $\mathrm{Et}_{2} \mathrm{O}$, the combined organic layers were dried over $\mathrm{MgSO}_{4}$ and concentrated under reduced pressure. The residue was quickly purified by flash column chromatography $\left(\mathrm{SiO}_{2}\right.$, hexane-EtOAc $=3: 1)$ to give aryl amine $\mathbf{1 0}$ (42.9 $\mathrm{mg}, 0.248 \mathrm{mmol}, 83 \%)$.

Yellow oil; ${ }^{1} \mathrm{H}$ NMR $\left(500 \mathrm{MHz}, \mathrm{CD}_{3} \mathrm{OD}\right) \delta 7.84(1 \mathrm{H}, \mathrm{d}, J=8.1 \mathrm{~Hz}), 7.67(1 \mathrm{H}, \mathrm{d}, J=8.1 \mathrm{~Hz}), 7.43$ $(1 \mathrm{H}, \mathrm{d}, J=8.6 \mathrm{~Hz}), 7.40-7.33(1 \mathrm{H}, \mathrm{m}), 7.19-7.15(1 \mathrm{H}, \mathrm{m}), 7.08(1 \mathrm{H}, \mathrm{d}, J=8.6 \mathrm{~Hz}), 4.88(2 \mathrm{H}, \mathrm{s})$, $3.84(3 \mathrm{H}, \mathrm{s}) ;{ }^{13} \mathrm{C}$ NMR $\left(125 \mathrm{MHz}, \mathrm{CDCl}_{3}\right) \delta 139.12,135.57,128.75,128.45,128.01,126.17$, 124.75, 122.45, 119.91, 118.52, 59.76; IR (ATR) v 3384, 2937, 2838, 2455, 1600, 1370, 1254, 1081, 990, $808 \mathrm{~cm}^{-1}$; HRMS (FD) calcd for $\mathrm{C}_{11} \mathrm{H}_{11} \mathrm{NO}\left(\mathrm{M}^{+}\right)$: 173.0841, found: 173.0837.

\section{General Procedure for the Cyclization with TMSNTf $_{2}-i-P_{2}$ NEt System (method A)}

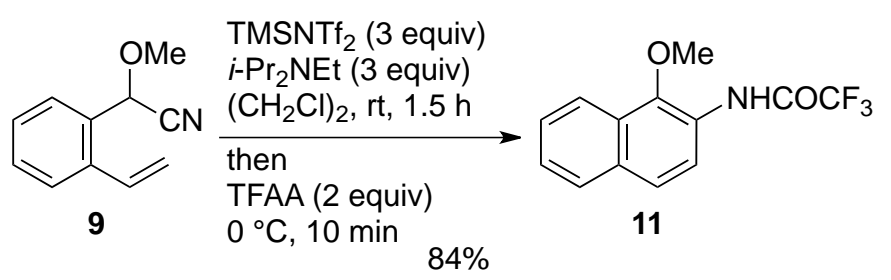

$\operatorname{TMSNTf}_{2}(206 \mu \mathrm{L}, 0.9 \mathrm{mmol}, 3$ equiv) was added to a solution of nitrile 9 (52.0 mg, $50 \mu \mathrm{L}, 0.300$ 
mmol, 1 equiv) and $i$ - $\operatorname{Pr}_{2} \mathrm{NEt}\left(147 \mu \mathrm{L}, 0.900 \mathrm{mmol}, 3\right.$ equiv) in $\left(\mathrm{CH}_{2} \mathrm{Cl}\right)_{2}(1.5 \mathrm{~mL})$, and the mixture was stirred at room temperature for $1.5 \mathrm{~h}$, at which point the consumption of starting material 9 was complete (as determined by TLC analysis, hexane-EtOAc $=4: 1$ ). After cooled to $0{ }^{\circ} \mathrm{C}$, TFAA ( $85 \mu \mathrm{L}, 0.600 \mathrm{mmol}, 2$ equiv) was added, and the mixture was stirred at $0{ }^{\circ} \mathrm{C}$ for 10 min. The reaction was quenched by slow addition of saturated aqueous $\mathrm{NaHCO}_{3}(3 \mathrm{~mL})$, and the resulting mixture was extracted with $\mathrm{Et}_{2} \mathrm{O}(2 \mathrm{~mL} \times 2)$. The combined organic extracts were dried over $\mathrm{MgSO}_{4}$ and concentrated under reduced pressure. The residue was purified by flash column chromatography $\left(\mathrm{SiO}_{2}\right.$, hexane-EtOAc $\left.=6: 1\right)$ to give aryl amine $11(67.8 \mathrm{mg}, 0.252 \mathrm{mmol}, 84 \%)$.

2,2,2-Trifluoro- $N$-(1-methoxynaphthalen-2-yl)acetamide1-Methoxynaphthalen-2-amine (11): White solid; M.p. $119-122{ }^{\circ} \mathrm{C} ;{ }^{1} \mathrm{H}$ NMR $\left(500 \mathrm{MHz}, \mathrm{CDCl}_{3}\right) \delta 8.66(1 \mathrm{H}, \mathrm{br}-\mathrm{s}), 8.41(1 \mathrm{H}, \mathrm{d}, J=9.2$ $\mathrm{Hz}), 8.04(1 \mathrm{H}, \mathrm{d}, J=8.1 \mathrm{~Hz}), 7.87(1 \mathrm{H}, \mathrm{d}, J=8.0 \mathrm{~Hz}), 7.70(1 \mathrm{H}, \mathrm{d}, J=9.2 \mathrm{~Hz}), 7.59-7.54(1 \mathrm{H}, \mathrm{m})$, 7.53-7.48 (1H, m), $4.00(3 \mathrm{H}, \mathrm{s}) ;{ }^{13} \mathrm{C} \mathrm{NMR}\left(125 \mathrm{MHz}, \mathrm{CDCl}_{3}\right) \delta 154.66\left[\mathrm{q},{ }^{2} J(\mathrm{C}, \mathrm{F})=37.0 \mathrm{~Hz}\right]$, $144.46,132.42,128.32,126.83,126.76,126.03,125.24,125.02,121.40,119.03,115.77$ [q, $\left.{ }^{1} J(\mathrm{C}, \mathrm{F})=287.3 \mathrm{~Hz}\right], 62.00$; IR (ATR) v 3271, 2355, 1709, 1541, 1375, 1340, 1257, 1153, 1087 , 991, 915, $814 \mathrm{~cm}^{-1}$; HRMS (FD) calcd for $\mathrm{C}_{13} \mathrm{H}_{10} \mathrm{~F}_{3} \mathrm{NO}_{2}\left(\mathrm{M}^{+}\right)$: 269.0664, found: 269.0654.

\section{General Procedure for the Cyclization with LDA-LDA System (method B)}

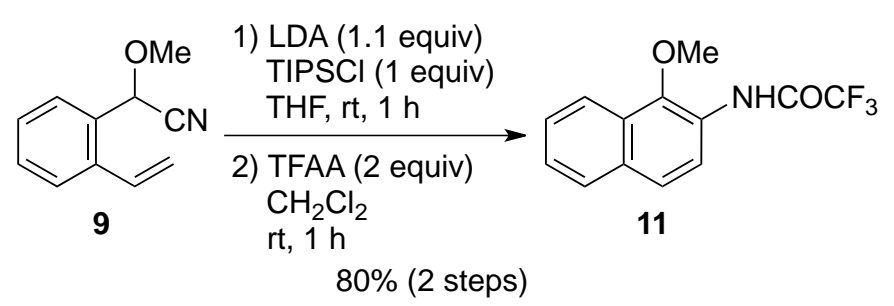

To a solution of $i-\operatorname{Pr}_{2} \mathrm{NH}(49 \mu \mathrm{L}, 0.348 \mathrm{mmol}, 1.16$ equiv) in THF (1.5 mL) was added $n$-BuLi (2.65 $\mathrm{M}$ in hexane, $125 \mu \mathrm{L}, 0.330 \mathrm{mmol}, 1.10$ equiv) at $-78^{\circ} \mathrm{C}$, and the mixture was stirred at this temperature for $15 \mathrm{~min}$. To the mixture was added nitrile $9(52.0 \mathrm{mg}, 50 \mu \mathrm{L}, 0.30 \mathrm{mmol}, 1$ equiv) at $-78{ }^{\circ} \mathrm{C}$, and the reaction mixture was stirred at this temperature for $1 \mathrm{~h}$. TIPSCl $(64 \mu \mathrm{L}, 0.300$ mmol, 1 equiv) was then added and the mixture was stirred at $-78{ }^{\circ} \mathrm{C}$ for $5 \mathrm{~min}$. After warmed to room temperature, the mixture was stirred at this temperature for $1 \mathrm{~h}$. The reaction was quenched by addition of saturated aqueous $\mathrm{NH}_{4} \mathrm{Cl}(3 \mathrm{~mL})$, and the resulting mixture was extracted with $\mathrm{Et}_{2} \mathrm{O}$ $(2 \mathrm{~mL} \times 2)$. The combined organic extracts were dried over $\mathrm{MgSO}_{4}$ and concentrated under reduced pressure. The crude aromatic amine $(116.2 \mathrm{mg})$ was used for the next step without further purification.

To a mixture of the above crude aromatic amine $(116.2 \mathrm{mg}$, as $0.300 \mathrm{mmol})$ in $\mathrm{CH}_{2} \mathrm{Cl}_{2}(1.5 \mathrm{~mL})$ was added TFAA ( $85 \mu \mathrm{L}, 0.600 \mathrm{mmol}, 2$ equiv) at $0{ }^{\circ} \mathrm{C}$ and the reaction mixture was stirred at room temperature for $1 \mathrm{~h}$. The reaction was quenched by slow addition of saturated aqueous $\mathrm{NaHCO}_{3}(3 \mathrm{~mL})$, and the resultant solution was extracted with $\mathrm{Et}_{2} \mathrm{O}(2 \mathrm{~mL} \times 2)$. The combined organic extracts were dried over $\mathrm{MgSO}_{4}$ and concentrated under reduced pressure. The residue was purified by flash column chromatography $\left(\mathrm{SiO}_{2}\right.$, hexane-EtOAc $\left.=6: 1\right)$ to give aryl amine 11 
(64.3 mg, $0.239 \mathrm{mmol}, 80 \%$ for 2 steps). ${ }^{1} \mathrm{H}$ and ${ }^{13} \mathrm{C}$ NMR spectra of this product were identical with those prepared by method A as mentioned above.

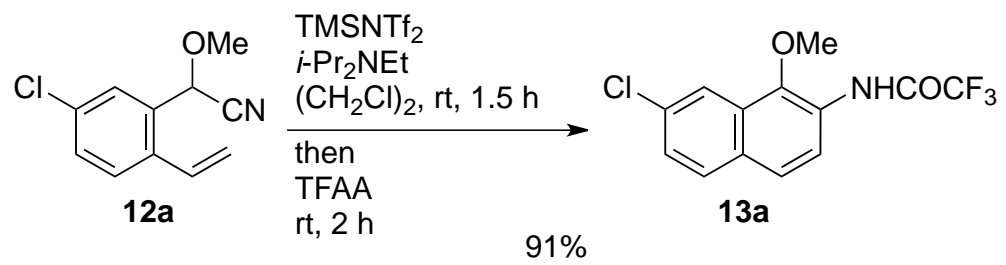

$N$-(7-Chloro-1-methoxynaphthalen-2-yl)-2,2,2-trifluoroacetamide (13a): Prepared by using the general procedure above (method A) from 2-(5-chloro-2-vinylphenyl)-2-methoxyacetonitrile (12a) $(62.3 \mathrm{mg}, 0.300 \mathrm{mmol})$ except for the reaction conditions of amidation (room temperature for $2 \mathrm{~h}$ ). Purification by flash column chromatography $\left(\mathrm{SiO}_{2}\right.$, hexane- $\left.\mathrm{Et}_{2} \mathrm{O}=15: 1\right)$ afforded aryl amine 13a (82.8 mg, $0.273 \mathrm{mmol}, 91 \%)$.

White powder; M.p. $130-135{ }^{\circ} \mathrm{C} ;{ }^{1} \mathrm{H}$ NMR $\left(500 \mathrm{MHz}, \mathrm{CDCl}_{3}\right) \delta 8.63(1 \mathrm{H}, \mathrm{br}-\mathrm{s}), 8.42(1 \mathrm{H}, \mathrm{d}, J=$ $9.2 \mathrm{~Hz}), 8.00(1 \mathrm{H}, \mathrm{d}, J=2.3 \mathrm{~Hz}), 7.80(1 \mathrm{H}, \mathrm{d}, J=9.2 \mathrm{~Hz}), 7.68(1 \mathrm{H}, \mathrm{d}, J=9.2 \mathrm{~Hz}), 7.43(1 \mathrm{H}, \mathrm{dd}$, $J=9.2,1.7 \mathrm{~Hz}), 3.99(3 \mathrm{H}, \mathrm{s}) ;{ }^{13} \mathrm{C} \mathrm{NMR}\left(125 \mathrm{MHz}, \mathrm{CDCl}_{3}\right) \delta 154.73\left[\mathrm{q},{ }^{2} J(\mathrm{C}, \mathrm{F})=37.0 \mathrm{~Hz}\right]$, $143.57,133.08,130.48,129.98,127.59,127.02,126.28,124.95,120.43,119.25,115.66$ [q, $\left.{ }^{1} J(\mathrm{C}, \mathrm{F})=287.4 \mathrm{~Hz}\right], 62.15$; IR (ATR) v 3409, 2941, 1722, 1529, 1504, 1270, 1148, 970, 830, 653 $\mathrm{cm}^{-1}$; HRMS (FD) calcd for $\mathrm{C}_{13} \mathrm{H}_{9} \mathrm{ClF}_{3} \mathrm{NO}_{2}\left(\mathrm{M}^{+}\right)$: 303.0274, found: 303.0269 .

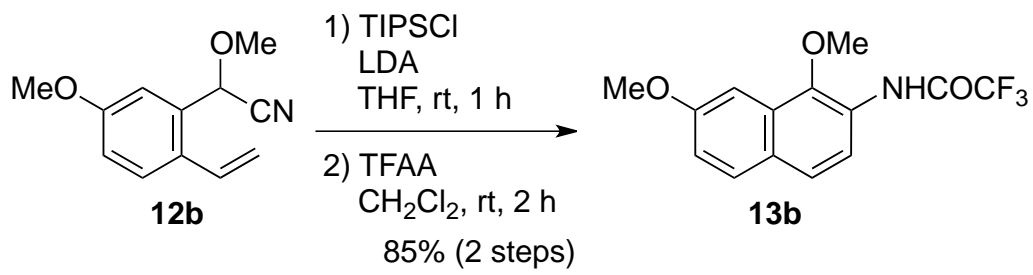

$N$-(1,7-Dimethoxynaphthalen-2-yl)-2,2,2-trifluoroacetamide (13b): Prepared by using the general procedure above (method B) from 2-methoxy-2-(5-methoxy-2-vinylphenyl)acetonitrile (12b) (40.6 mg, $0.200 \mathrm{mmol}$ ) except for the reaction conditions for amidation (TFAA (28 $\mu \mathrm{L}, 0.20$ mmol, 1 equiv), room temperature for $2 \mathrm{~h})$. Purification by flash column chromatography $\left(\mathrm{SiO}_{2}\right.$, hexane- $\left.\mathrm{Et}_{2} \mathrm{O}=10: 1\right)$ afforded aryl amine $\mathbf{1 3 b}(51.5 \mathrm{mg}, 0.171 \mathrm{mmol}, 85 \%$ for 2 steps).

White solid; M.p. $71-73{ }^{\circ} \mathrm{C} ;{ }^{1} \mathrm{H}$ NMR $\left(500 \mathrm{MHz}, \mathrm{CDCl}_{3}\right) \delta 8.64(1 \mathrm{H}, \mathrm{br}-\mathrm{s}), 8.25(1 \mathrm{H}, \mathrm{d}, J=8.6$ $\mathrm{Hz}), 7.75(1 \mathrm{H}, \mathrm{d}, J=8.2 \mathrm{~Hz}), 7.61(1 \mathrm{H}, \mathrm{d}, J=8.6 \mathrm{~Hz}), 7.27(1 \mathrm{H}, \mathrm{d}, J=2.3 \mathrm{~Hz}), 7.15(1 \mathrm{H}, \mathrm{dd}, J=$ 9.2, $2.3 \mathrm{~Hz}), 3.98(3 \mathrm{H}, \mathrm{s}), 3.97(3 \mathrm{H}, \mathrm{s}) ;{ }^{13} \mathrm{C} \mathrm{NMR}\left(125 \mathrm{MHz}, \mathrm{CDCl}_{3}\right) \delta 158.54,154.61$ [q, ${ }^{2} J(\mathrm{C}, \mathrm{F})$ $=37.0 \mathrm{~Hz}], 143.50,130.00,128.02,127.96,125.84,124.80,118.89,116.49,115.77\left[\mathrm{q},{ }^{1} J(\mathrm{C}, \mathrm{F})=\right.$ $287.3 \mathrm{~Hz}$ ], 99.49 61.40, 55.38; IR (ATR) v 3284, 1710, 1541, 1074, 1031, 979, 833, 771, 683 $\mathrm{cm}^{-1}$; HRMS (FD) calcd for $\mathrm{C}_{14} \mathrm{H}_{12} \mathrm{~F}_{3} \mathrm{NO}_{3}\left(\mathrm{M}^{+}\right)$: 299.0769, found: 299.0776. 
<smiles>C=C(C)c1ccccc1C(C)OC</smiles>

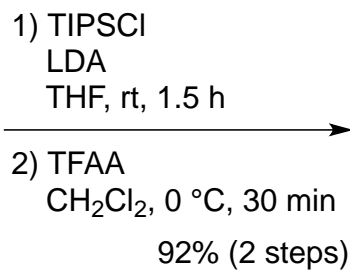<smiles>COc1c(NC(C)C(F)(F)F)cc(C)c2ccccc12</smiles>

2,2,2-Trifluoro- $N$-(1-methoxy-4-methylnaphthalen-2-yl)acetamide (13c): Prepared by using the general procedure above (method B) from 2-methoxy-2-(2-(prop-1-en-2-yl)phenyl)acetonitrile (12c) $(44.9 \mathrm{mg}, 0.240 \mathrm{mmol})$ except for the reaction time of cyclization $(1.5 \mathrm{~h})$ and the reaction conditions of amidation $\left(0{ }^{\circ} \mathrm{C}\right.$ for $\left.30 \mathrm{~min}\right)$. Purification by flash column chromatography $\left(\mathrm{SiO}_{2}\right.$, hexane- $\left.\mathrm{Et}_{2} \mathrm{O}=10: 1\right)$ afforded aryl amine $13 \mathrm{c}(62.5 \mathrm{mg}, 0.221 \mathrm{mmol}, 92 \%$ for 2 steps $)$.

White solid; M.p. $102-104{ }^{\circ} \mathrm{C} ;{ }^{1} \mathrm{H}$ NMR (500 MHz, $\left.\mathrm{CDCl}_{3}\right) \delta 8.60$ (1H, br-s), 8.25 (1H, s), 8.05 $(1 \mathrm{H}, \mathrm{d}, J=8.0 \mathrm{~Hz}), 7.99(1 \mathrm{H}, \mathrm{d}, J=8.6 \mathrm{~Hz}), 7.60-7.51(2 \mathrm{H}, \mathrm{m}), 3.97(3 \mathrm{H}, \mathrm{s}), 2.69(3 \mathrm{H}, \mathrm{s}) ;{ }^{13} \mathrm{C}$ NMR $\left(125 \mathrm{MHz}, \mathrm{CDCl}_{3}\right) \delta 155.12$ [q, $\left.{ }^{2} J(\mathrm{C}, \mathrm{F})=37.0 \mathrm{~Hz}\right], 143.02,131.91,131.42,126.89,126.47$, 125.86, 124.83, 124.61, 121.91, 119.38, 115.80 [q, $\left.{ }^{1} J(\mathrm{C}, \mathrm{F})=286.2 \mathrm{~Hz}\right], 62.05,19.24$; IR (ATR) $v$ 3202, 1537, 1335, 1244, 1155, 1102, 926, $768 \mathrm{~cm}^{-1}$; HRMS (FD) calcd for $\mathrm{C}_{14} \mathrm{H}_{12} \mathrm{~F}_{3} \mathrm{NO}_{2}$ $\left(\mathrm{M}^{+}\right): 283.0820$, found: 283.0824 .<smiles>COC(C#N)c1ccccc1/C=C\c1ccccc1</smiles>

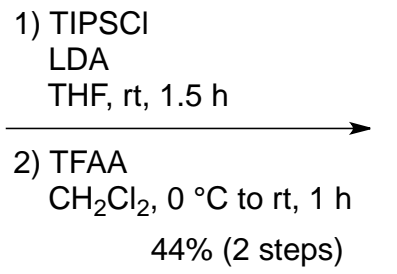<smiles>COc1c(NC(=O)C(F)(F)F)c(-c2ccccc2)cc2ccccc12</smiles>

2,2,2-Trifluoro- $N$-(1-methoxy-3-phenylnaphthalen-2-yl)acetamide (13d): Prepared by using the general procedure above (method B) from $(E)$-2-methoxy-2-(2-styrylphenyl)acetonitrile (12d) (93.6 $\mathrm{mg}, 0.375 \mathrm{mmol})$ except for the reaction time of cyclization $(1.5 \mathrm{~h})$ and the reaction conditions of amidation $\left(0{ }^{\circ} \mathrm{C}\right.$ for $30 \mathrm{~min}$ then room temperature for $\left.30 \mathrm{~min}\right)$. Purification by flash column chromatography $\left(\mathrm{SiO}_{2}\right.$, hexane-EtOAc $\left.=15: 1\right)$ afforded aryl amine 13d $(56.8 \mathrm{mg}, 0.164$ mmol, $44 \%$ for 2 steps).

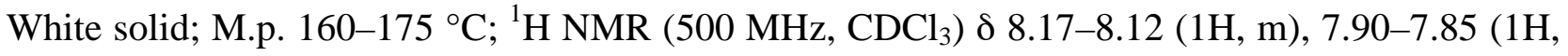
m), $7.79(1 \mathrm{H}, \mathrm{s}), 7.66(1 \mathrm{H}, \mathrm{s}), 7.61-7.55(2 \mathrm{H}, \mathrm{m}), 7.44-7.34(5 \mathrm{H}, \mathrm{m}), 3.96(3 \mathrm{H}, \mathrm{s}) ;{ }^{13} \mathrm{C} \mathrm{NMR}(125$ $\left.\mathrm{MHz}, \mathrm{CDCl}_{3}\right) \delta 155.90$ [q, $\left.{ }^{2} \mathrm{~J}(\mathrm{C}, \mathrm{F})=37.0 \mathrm{~Hz}\right], 151.91,138.10,137.86,134.06,128.55,128.44$, $128.25,127.84,127.41,127.15,126.60,125.53,122.30,121.16,115.81$ [q, ${ }^{1} J(C, F)=287.3 \mathrm{~Hz}$, 62.12; IR (ATR) v 3229, 3057, 2980, 1708, 1547, 1375, 1262, 752, $696 \mathrm{~cm}^{-1}$; HRMS (FD) calcd for $\mathrm{C}_{19} \mathrm{H}_{14} \mathrm{~F}_{3} \mathrm{NO}_{2}\left(\mathrm{M}^{+}\right)$: 345.0977, found: 345.0985 . 


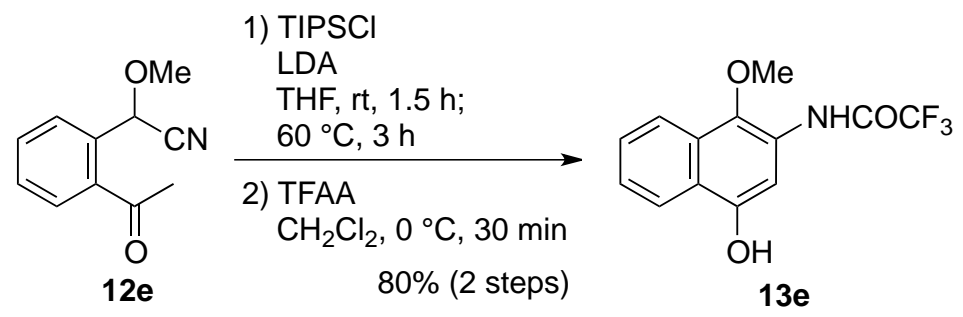

2,2,2-Trifluoro- $N$-(4-hydroxy-1-methoxynaphthalen-2-yl)acetamide (13e): To a solution of $i$ - $\operatorname{Pr}_{2} \mathrm{NH}(93 \mu \mathrm{L}, 0.66 \mathrm{mmol}, 2.2$ equiv) in THF $(1.5 \mathrm{~mL})$ was added $n$-BuLi (2.65 $\mathrm{M}$ in hexane, $238 \mu \mathrm{L}, 0.63 \mathrm{mmol}, 2.1$ equiv) at $-78{ }^{\circ} \mathrm{C}$, and the mixture was stirred at this temperature for 15 min. Nitrile 12e $(56.8 \mathrm{mg}, 0.300 \mathrm{mmol})$ in THF $(0.5 \mathrm{~mL})$ was then added, and the mixture was stirred at $-78{ }^{\circ} \mathrm{C}$ for $1 \mathrm{~h}$. After addition of TIPSCl $(127 \mu \mathrm{L}, 0.90 \mathrm{mmol}, 2$ equiv) and stirred at $-78{ }^{\circ} \mathrm{C}$ for $5 \mathrm{~min}$, the mixture was allowed to warm to room temperature. The mixture wad stirred at room temperature for $1.5 \mathrm{~h}$ and then at $60{ }^{\circ} \mathrm{C}$ for $3 \mathrm{~h}$. The reaction was quenched by addition of saturated aqueous $\mathrm{NH}_{4} \mathrm{Cl}(3 \mathrm{~mL})$, and the resulting mixture was extracted with $\mathrm{Et}_{2} \mathrm{O}(2 \mathrm{~mL} \times 2)$. The combined organic extracts were dried over $\mathrm{MgSO}_{4}$ and concentrated under reduced pressure. The crude aromatic amine (132.8 $\mathrm{mg}$ ) was used for the next step without further purification.

To a mixture of the above crude aromatic amine $(132.8 \mathrm{mg}$, as $0.300 \mathrm{mmol})$ in $\mathrm{CH}_{2} \mathrm{Cl}_{2}(1.5 \mathrm{~mL})$ was added TFAA (127 $\mu \mathrm{L}, 0.900 \mathrm{mmol}, 3$ equiv), and the reaction mixture was stirred at room temperature for $30 \mathrm{~min}$. The reaction was quenched by slow addition of saturated aqueous $\mathrm{NaHCO}_{3}(3 \mathrm{~mL})$, and the resulting mixture was extracted with $\mathrm{Et}_{2} \mathrm{O}(2 \mathrm{~mL} \times 2)$. The combined organic extracts were dried over $\mathrm{MgSO}_{4}$ and concentrated under reduced pressure. The residue was purified by flash column chromatography $\left(\mathrm{SiO}_{2}\right.$, hexane- $\left.\mathrm{Et}_{2} \mathrm{O}=5: 1\right)$ to give aryl amine 13e (68.4 mg, $0.240 \mathrm{mmol}, 80 \%$ for 2 steps).

White solid; M.p. $164-167{ }^{\circ} \mathrm{C} ;{ }^{1} \mathrm{H}$ NMR $\left(500 \mathrm{MHz}, \mathrm{CD}_{3} \mathrm{CN}\right) \delta 8.99(1 \mathrm{H}, \mathrm{br}-\mathrm{s}), 8.14(1 \mathrm{H}, \mathrm{d}, J=$ $8.6 \mathrm{~Hz}), 8.01(1 \mathrm{H}, \mathrm{d}, J=8.0 \mathrm{~Hz}), 7.80(1 \mathrm{H}, \mathrm{br}-\mathrm{s}), 7.59-7.93(1 \mathrm{H}, \mathrm{m}), 7.54(1 \mathrm{H}, \mathrm{s}), 7.51-7.45(1 \mathrm{H}$, m), $3.86(3 \mathrm{H}, \mathrm{s}) ;{ }^{13} \mathrm{C} \mathrm{NMR}\left(125 \mathrm{MHz}, \mathrm{CD}_{3} \mathrm{CN}\right) \delta 155.98$ [q, $\left.{ }^{2} J(\mathrm{C}, \mathrm{F})=37.0 \mathrm{~Hz}\right], 150.23,140.54$, $128.88,128.21,126.25,125.57,124.67,123.46,122.48,117.97$ [q, $\left.{ }^{1} J(\mathrm{C}, \mathrm{F})=285.0 \mathrm{~Hz}\right], 103.88$, 62.71; IR (ATR) v 3332, 2957, 1707, 1594, 1542, 1441, 1388, 1312, 1260, 1146, 1075, 990, 960, $909,848,763,650 \mathrm{~cm}^{-1}$; HRMS (FD) calcd for $\mathrm{C}_{13} \mathrm{H}_{10} \mathrm{~F}_{3} \mathrm{NO}_{3}\left(\mathrm{M}^{+}\right)$: 285.0613, found: 285.0621 .

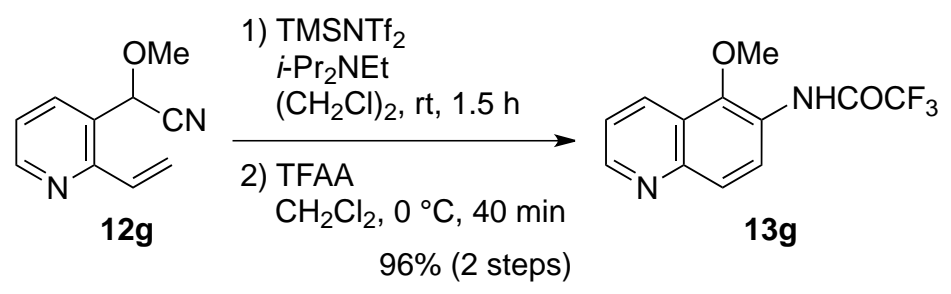

2,2,2-Trifluoro- $N$-(5-methoxyquinolin-6-yl)acetamide (13g): $\operatorname{TMSNTf}_{2}$ (206 $\mu \mathrm{L}, 0.9$ mmol, 3equiv) was added to a solution of nitrile $\mathbf{1 2 g}\left(52.3 \mathrm{mg}, 0.300 \mathrm{mmol}, 1\right.$ equiv) and $i-\operatorname{Pr}_{2} \mathrm{NEt}$ (147 $\mu \mathrm{L}, 0.900 \mathrm{mmol}, 3$ equiv) in $\left(\mathrm{CH}_{2} \mathrm{Cl}\right)_{2}(1.5 \mathrm{~mL})$, and the mixture was stirred at room temperature 


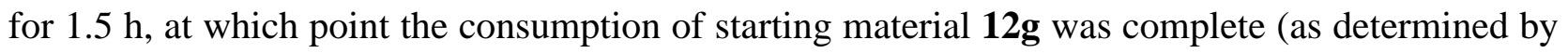
TLC analysis, hexane-EtOAc $=1: 1)$. The reaction was quenched by slow addition of saturated aqueous $\mathrm{NaHCO}_{3}(3 \mathrm{~mL})$, and the resulting mixture was extracted with EtOAc $(2 \mathrm{~mL} \times 2)$. The combined organic extracts were dried over $\mathrm{MgSO}_{4}$ and concentrated under reduced pressure. The crude aromatic amine (325.4 mg) was used for the next step without further purification.

TFAA (42 $\mu \mathrm{L}, 0.300 \mathrm{mmol}, 1$ equiv) was added to a solution of the above crude aromatic amine (325.4 mg, as $0.300 \mathrm{mmol}$ ) in $\mathrm{CH}_{2} \mathrm{Cl}_{2}(1.5 \mathrm{~mL})$, and the mixture was stirred at $0{ }^{\circ} \mathrm{C}$ for $40 \mathrm{~min}$. The reaction was quenched by slow addition of saturated aqueous $\mathrm{NaHCO}_{3}(3 \mathrm{~mL})$, and the resulting mixture was extracted with $\mathrm{Et}_{2} \mathrm{O}(2 \mathrm{~mL} \times 2)$. The combined organic extracts were dried over $\mathrm{MgSO}_{4}$ and concentrated under reduced pressure. The residue was purified by flash column chromatography $\left(\mathrm{SiO}_{2}\right.$, hexane-EtOAc $\left.=1: 1\right)$ to give aryl amine $\mathbf{1 3 g}(78.0 \mathrm{mg}, 0.289 \mathrm{mmol}, 96 \%$ for 2 steps).

Green solid; M.p. $164-177{ }^{\circ} \mathrm{C} ;{ }^{1} \mathrm{H}$ NMR $\left(500 \mathrm{MHz}, \mathrm{CDCl}_{3}\right) \delta 8.94(1 \mathrm{H}, \mathrm{dd}, J=4.3,1.4 \mathrm{~Hz}), 8.68$ $(1 \mathrm{H}, \mathrm{dd}, J=9.2,2.9 \mathrm{~Hz}), 8.60(1 \mathrm{H}, \mathrm{br}-\mathrm{s}), 8.41(1 \mathrm{H}, \mathrm{d}, J=8.6 \mathrm{~Hz}), 8.02(1 \mathrm{H}, \mathrm{d}, J=9.8 \mathrm{~Hz}), 7.51$ $(1 \mathrm{H}, \mathrm{dd}, J=8.3,4.3 \mathrm{~Hz}), 4.01(3 \mathrm{H}, \mathrm{s}) ;{ }^{13} \mathrm{C} \mathrm{NMR}\left(125 \mathrm{MHz}, \mathrm{CDCl}_{3}\right) \delta 154.85\left[\mathrm{q},{ }^{2} J(\mathrm{C}, \mathrm{F})=38.2\right.$ $\mathrm{Hz}], 150.14,146.27,144.21,130.32,126.21,125.74,122.86,122.32,121.54,115.61$ [q, ${ }^{1} J(\mathrm{C}, \mathrm{F})=$ $287.4 \mathrm{~Hz}$; IR (ATR) v 3315, 2925, 1711, 1542, 1323, 1265, 1153, 1075, 831, 812, $663 \mathrm{~cm}^{-1}$; HRMS (FD) calcd for $\mathrm{C}_{12} \mathrm{H}_{9} \mathrm{~F}_{3} \mathrm{~N}_{2} \mathrm{O}_{2}\left(\mathrm{M}^{+}\right)$: 270.0616, found: 270.0595 .

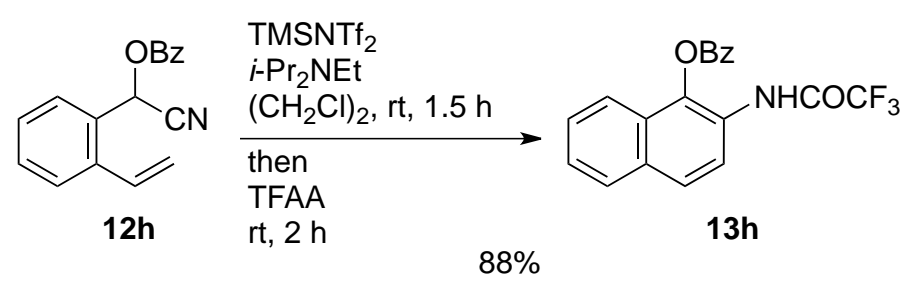

2-(2,2,2-Trifluoroacetamido)naphthalen-1-yl benzoate (13h): Prepared by using the general procedure above (method A) from cyano(2-vinylphenyl)methyl benzoate (12h) $(79.0 \mathrm{mg}, 0.300$ mmol) except for the reaction time of amidation $(2 \mathrm{~h})$. Purification by flash column chromatography $\left(\mathrm{SiO}_{2}\right.$, hexane-EtOAc $\left.=4: 1\right)$ afforded aryl amine 13h $(94.9 \mathrm{mg}, 0.264 \mathrm{mmol}$, $88 \%$ ).

White solid; M.p. $182-185{ }^{\circ} \mathrm{C} ;{ }^{1} \mathrm{H}$ NMR $\left(500 \mathrm{MHz}, \mathrm{CDCl}_{3}\right) \delta 8.36(2 \mathrm{H}, \mathrm{d}, J=6.9 \mathrm{~Hz}), 8.20(1 \mathrm{H}$, br-s), $8.10(1 \mathrm{H}, \mathrm{d}, J=9.2 \mathrm{~Hz}), 7.94-7.90(1 \mathrm{H}, \mathrm{m}), 7.88(2 \mathrm{H}, \mathrm{d}, J=9.2 \mathrm{~Hz}), 7.77(1 \mathrm{H}, \mathrm{t}, J=7.7$ $\mathrm{Hz}), 7.63(2 \mathrm{H}, \mathrm{t}, J=7.7 \mathrm{~Hz}), 7.68-7.53(2 \mathrm{H}, \mathrm{m}) ;{ }^{13} \mathrm{C} \mathrm{NMR}\left(125 \mathrm{MHz}, \mathrm{CDCl}_{3}\right) \delta 164.50,155.21[\mathrm{q}$, $\left.{ }^{2} J(\mathrm{C}, \mathrm{F})=38.2 \mathrm{~Hz}\right], 137.85,134.71,132.77,130.55,129.09,128.11,127.67,127.43,127.10$, 126.92, 126.81, 124.21, 121.45, 121.28, 115.62 [q, $\left.{ }^{1} J(\mathrm{C}, \mathrm{F})=287.3 \mathrm{~Hz}\right]$; IR (ATR) v 3237, 1739, 1710, 1544, 1245, 1158, 1085, 1047, 1021, 908, 806, $706 \mathrm{~cm}^{-1}$; HRMS (FD) calcd for $\mathrm{C}_{19} \mathrm{H}_{12} \mathrm{~F}_{3} \mathrm{NO}_{3}\left(\mathrm{M}^{+}\right):$359.0769, found: 359.0785 . 


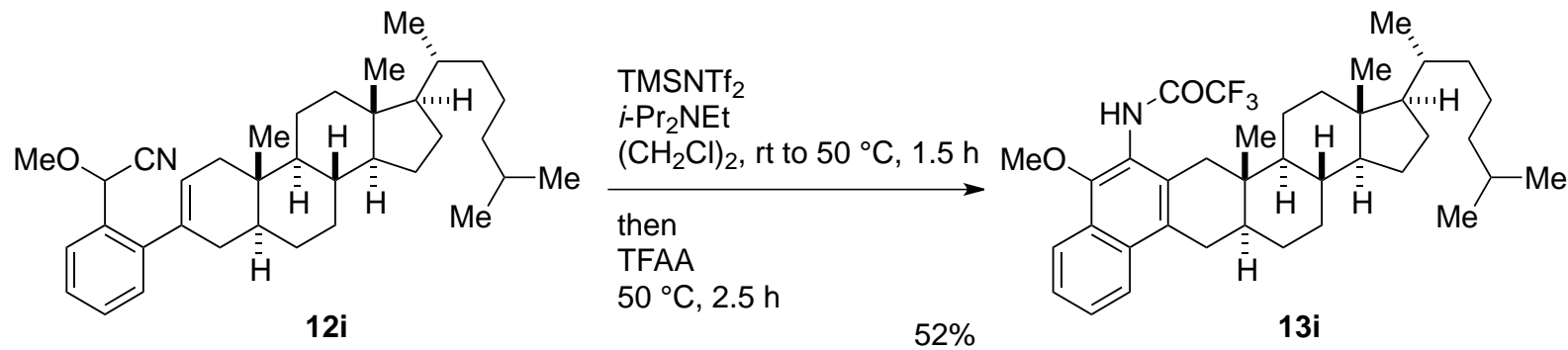

2,2,2-Trifluoro- $N-((1 R, 3 \mathrm{a} S, 3 \mathrm{~b} R, 5 \mathrm{a} S, 13 \mathrm{a} S, 13 \mathrm{~b} S, 15 \mathrm{a} R)-11-m e t h o x y-13 \mathrm{a}, 15 \mathrm{a}-$ dimethyl-1-( $(R)-6-$ methylheptan-2-yl)-2,3,3a,3b,4,5,5a,6,13,13a,13b,14,15,15a-tetradecahydro-1H-benzo[k]cyclo penta[c]tetraphen-12-yl)acetamide (13i): Prepared by using the general procedure above (method A) from nitrile $12 \mathbf{i}(61.8 \mathrm{mg}, 0.120 \mathrm{mmol})$ except for the reaction conditions of cyclization (room temperature for $30 \mathrm{~min}$ then $50{ }^{\circ} \mathrm{C}$ for $1 \mathrm{~h}$ ) and the reaction conditions of amidation $\left(50{ }^{\circ} \mathrm{C}\right.$ for $\left.2.5 \mathrm{~h}\right)$. Purification by flash column chromatography $\left(\mathrm{SiO}_{2}\right.$, hexane-EtOAc $=$ 10:1) afforded aryl amine $\mathbf{1 3 i}(38.2 \mathrm{mg}, 0.0624 \mathrm{mmol}, 52 \%)$.

Colorless oil; $[\alpha]_{\mathrm{D}}{ }^{23} 4.33$ (c 4.32, $\left.\mathrm{CHCl}_{3}\right) ;{ }^{1} \mathrm{H}$ NMR $\left(500 \mathrm{MHz}, \mathrm{CDCl}_{3}\right) \delta 8.49(1 \mathrm{H}, \mathrm{d}, J=8.6 \mathrm{~Hz})$, $8.00(1 \mathrm{H}, \mathrm{d}, J=8.0 \mathrm{~Hz}), 7.95(1 \mathrm{H}, \mathrm{s}), 7.58-7.47(2 \mathrm{H}, \mathrm{m}), 3.90(3 \mathrm{H}, \mathrm{s}), 3.06(1 \mathrm{H}, \mathrm{dd}, J=17.2,5.2$ Hz), 2.66-2.54 (2H, m), 2.29 (1H, d, $J=16.7 \mathrm{~Hz}), 2.03$ (1H, d, $J=12.6 \mathrm{~Hz}), 1.89-1.79$ (1H, m), $1.75(1 \mathrm{H}, \mathrm{d}, J=12.6 \mathrm{~Hz}), 1.68(1 \mathrm{H}, J=13.2 \mathrm{~Hz}), 1.64-1.44(6 \mathrm{H}, \mathrm{m}), 1.44-0.95(13 \mathrm{H}, \mathrm{m}), 0.93$ $(3 \mathrm{H}, \mathrm{d}, J=6.3 \mathrm{~Hz}), 0.90-0.78(8 \mathrm{H}, \mathrm{m}), 0.67(3 \mathrm{H}, \mathrm{s}), 0.64(3 \mathrm{H}, \mathrm{s}) ;{ }^{13} \mathrm{C} \mathrm{NMR}\left(125 \mathrm{MHz}, \mathrm{CDCl}_{3}\right) \delta$ $156.44\left[\mathrm{q},{ }^{2} J(\mathrm{C}, \mathrm{F})=37.0 \mathrm{~Hz}\right], 149.48,132.69,131.41,128.73,126.89,125.95,125.54,123.58$, 122.76, 122.36, 116.14 [q, ${ }^{1} J(\mathrm{C}, \mathrm{F})=286.2 \mathrm{~Hz}$ ] $62.28,56.25,53.45,42.39,40.95,39.90,39.51$, $39.28,36.17,35.80,35.49,34.32,31.61,30.88,28.82,28.22,28.02,24.24,23.85,22.83,22.57$, 20.96, 18.68, 11.92, 11.36; IR (ATR) v 2929, 1707, 1559, 1456, 1377, 1339, 1219, 1186, 772 $\mathrm{cm}^{-1}$; HRMS (FD) calcd for $\mathrm{C}_{38} \mathrm{H}_{52} \mathrm{~F}_{3} \mathrm{NO}_{2}\left(\mathrm{M}^{+}\right)$: 611.3950, found: 611.3950 .

\section{Synthesis of Aryl Amines 15a-f (Scheme 7)}

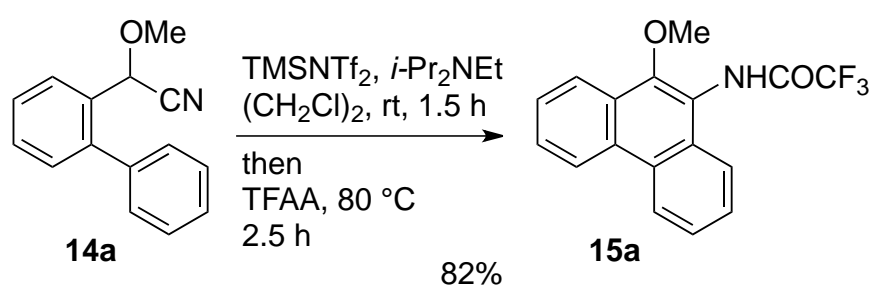

2,2,2-Trifluoro- $N$-(10-methoxyphenanthren-9-yl)acetamide (15a): Prepared by using the general procedure above (method A) from 2-([1,1'-biphenyl]-2-yl)-2-methoxyacetonitrile (14a) $(67.0 \mathrm{mg}, 0.300 \mathrm{mmol})$ except for the reaction conditions of amidation $\left(80{ }^{\circ} \mathrm{C}\right.$ for $\left.2.5 \mathrm{~h}\right)$. Purification by flash column chromatography $\left(\mathrm{SiO}_{2}\right.$, hexane-EtOAc $\left.=6: 1\right)$ afforded aryl amine 15a (78.9 mg, $0.247 \mathrm{mmol}, 82 \%)$.

White solid; M.p. $185-187{ }^{\circ} \mathrm{C} ;{ }^{1} \mathrm{H}$ NMR $\left(500 \mathrm{MHz},\left(\mathrm{CD}_{3}\right)_{2} \mathrm{CO}\right) \delta 10.33(1 \mathrm{H}, \mathrm{br}-\mathrm{s}), 8.88(1 \mathrm{H}, \mathrm{d}, J$ $=8.0 \mathrm{~Hz}), 8.88-8.82(1 \mathrm{H}, \mathrm{m}), 8.27(1 \mathrm{H}, \mathrm{d}, J=8.1 \mathrm{~Hz}), 7.98-7.93(1 \mathrm{H}, \mathrm{m}), 7.82-7.77(1 \mathrm{H}, \mathrm{m})$, 
7.77-7.72 (1H, m), 7.72-7.67 (2H, m), $4.01(3 \mathrm{H}, \mathrm{s}) ;{ }^{13} \mathrm{C} \mathrm{NMR}\left(125 \mathrm{MHz},\left(\mathrm{CD}_{3}\right)_{2} \mathrm{SO}\right) \delta 156.47$ [q, $\left.{ }^{2} J(\mathrm{C}, \mathrm{F})=37.0 \mathrm{~Hz}\right], 15.054,130.96,129.25,128.31,128.03,127.94,127.75,127.11,126.73$, 123.69, 123.57, 123.10, 119.67, 116.48 [q, $\left.{ }^{1} J(\mathrm{C}, \mathrm{F})=287.3 \mathrm{~Hz}\right], 61.79$; IR (ATR) v 3240, 1543, $1450,1325,1204,1156,1105,1029,969,936,756 \mathrm{~cm}^{-1}$; HRMS (FD) calcd for $\mathrm{C}_{17} \mathrm{H}_{12} \mathrm{~F}_{3} \mathrm{NO}_{2}$ $\left(\mathrm{M}^{+}\right)$: 319.0820, found: 319.0820 .

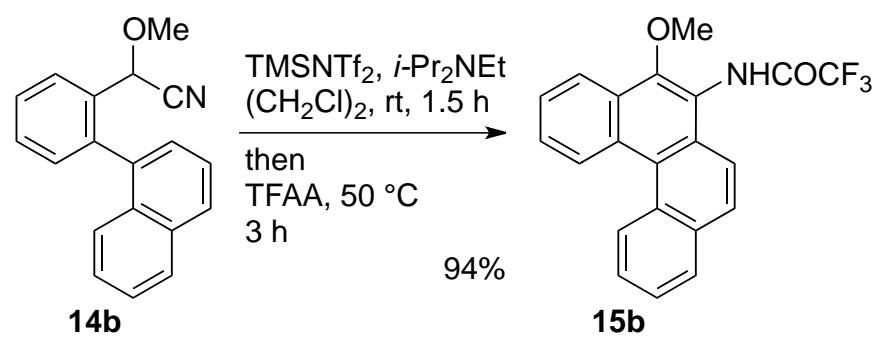

2,2,2-Trifluoro- $N$-(5-methoxybenzo[ $c$ ]phenanthren-6-yl)acetamide (15b): Prepared by using the general procedure above (method A) from 2-methoxy-2-(2-(naphthalen-1-yl)phenyl)acetonitrile (14b) $(68.0 \mathrm{mg}, 0.249 \mathrm{mmol})$ except for the reaction conditions of amidation (TFAA (140 $\mu \mathrm{L}, 0.996 \mathrm{mmol}, 4$ equiv), $50{ }^{\circ} \mathrm{C}$ for $3 \mathrm{~h}$ ). Purification by flash column chromatography $\left(\mathrm{SiO}_{2}\right.$, hexane-EtOAc $\left.=6: 1\right)$ afforded aryl amine 15b (86.3 mg, 0.234 mmol, 94\%).

White solid; M.p. $160-173{ }^{\circ} \mathrm{C} ;{ }^{1} \mathrm{H}$ NMR $\left(500 \mathrm{MHz}, \mathrm{CDCl}_{3}\right) \delta 9.10(1 \mathrm{H}, \mathrm{d}, J=8.6 \mathrm{~Hz}), 9.02(1 \mathrm{H}$, d, $J=8.6 \mathrm{~Hz}), 8.34(1 \mathrm{H}, \mathrm{d}, J=8.1 \mathrm{~Hz}), 8.07(1 \mathrm{H}, \mathrm{br}-\mathrm{s}), 8.03(1 \mathrm{H}, \mathrm{d}, J=7.4 \mathrm{~Hz}), 7.95(1 \mathrm{H}, \mathrm{d}, J=$ $9.2 \mathrm{~Hz}), 7.78-7.67(4 \mathrm{H}, \mathrm{m}), 7.66-7.62(1 \mathrm{H}, \mathrm{m}), 4.02(3 \mathrm{H}, \mathrm{s}) ;{ }^{13} \mathrm{C} \mathrm{NMR}\left(125 \mathrm{MHz},\left(\mathrm{CD}_{3}\right)_{2} \mathrm{CO}\right) \delta$ $157.62\left[\mathrm{q},{ }^{2} J(\mathrm{C}, \mathrm{F})=37.0 \mathrm{~Hz}\right], 152.06,133.82,131.75,130.56,129.38,129.33,129.18,129.00$, $128.86,128.43,128.18,127.54,127.43,126.96,125.83,123.72,121.27,120.57,117.45$ [q, $\left.{ }^{1} J(\mathrm{C}, \mathrm{F})=286.2 \mathrm{~Hz}\right], 62.27$; IR $(\mathrm{ATR}) \vee 3257,2933,1713,1539,1219,1151,1122,1103,1072$ $\mathrm{cm}^{-1}$; HRMS (FD) calcd for $\mathrm{C}_{21} \mathrm{H}_{14} \mathrm{~F}_{3} \mathrm{NO}_{2}\left(\mathrm{M}^{+}\right)$: 369.0977, found: 369.0994 .
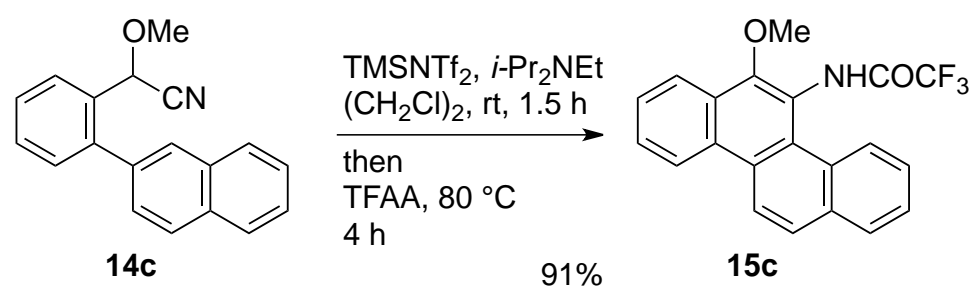

2,2,2-Trifluoro- $N$-(6-methoxychrysen-5-yl)acetamide (15c): Prepared by using the general procedure above (method A) from 2-methoxy-2-(2-(naphthalen-2-yl)phenyl)acetonitrile (14c) (82.0 $\mathrm{mg}, 0.300 \mathrm{mmol})$ except for the reaction conditions of amidation $\left(80{ }^{\circ} \mathrm{C}\right.$ for $\left.4 \mathrm{~h}\right)$. Purification by flash column chromatography $\left(\mathrm{SiO}_{2}\right.$, hexane- $\left.\mathrm{Et}_{2} \mathrm{O}=1: 1\right)$ afforded aryl amine 15c (100.6 mg, $0.272 \mathrm{mmol}, 91 \%)$.

White solid; M.p. $193-202{ }^{\circ} \mathrm{C} ;{ }^{1} \mathrm{H}$ NMR $\left(500 \mathrm{MHz}, \mathrm{CDCl}_{3}\right) \delta 8.90(1 \mathrm{H}, \mathrm{d}, J=8.6 \mathrm{~Hz}), 8.76(1 \mathrm{H}$, $\mathrm{d}, J=8.6 \mathrm{~Hz}), 8.63(1 \mathrm{H}, \mathrm{d}, J=9.2 \mathrm{~Hz}), 8.23(1 \mathrm{H}, \mathrm{d}, J=8.6 \mathrm{~Hz}), 7.98-7.92(2 \mathrm{H}, \mathrm{m}), 7.80-7.74$ 
(1H, m), 7.74-7.68 (1H, m), 7.63-7.53 (2H, m), 3.91 (3H, s); ${ }^{13} \mathrm{C}$ NMR (125 MHz, $\left.\left(\mathrm{CD}_{3}\right)_{2} \mathrm{CO}\right) \delta$ $156.97\left[\mathrm{q},{ }^{2} J(\mathrm{C}, \mathrm{F})=37.0 \mathrm{~Hz}\right], 153.51,134.26,132.33,130.53,129.74,129.00,128.65,128.25$, $128.19,127.68,127.54,127.04,126.88,126.34,124.92,123.85,122.78,122.05,117.47$ [q, $\left.{ }^{1} J(\mathrm{C}, \mathrm{F})=287.4 \mathrm{~Hz}\right], 62.34$; IR $(\mathrm{ATR}) \vee 3258,2162,1705,1684,1591,1533,1429,1339,1219$, $1155,772 \mathrm{~cm}^{-1}$; HRMS (FD) calcd for $\mathrm{C}_{21} \mathrm{H}_{14} \mathrm{~F}_{3} \mathrm{NO}_{2}\left(\mathrm{M}^{+}\right)$: 369.0977 , found: 369.0979 .

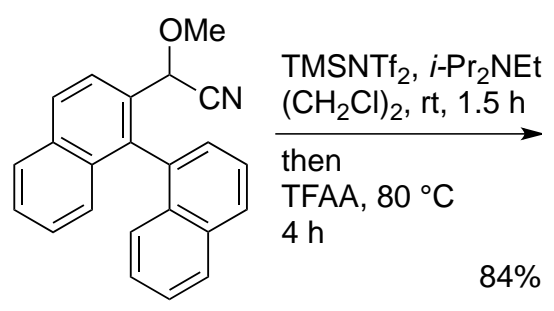

14d<smiles>COc1c(NC(=O)C(F)(F)F)c2ccc3ccccc3c2c2c1ccc1ccccc12</smiles>

$15 d$

2,2,2-Trifluoro- $N$-(4-methoxydibenzo[c,g]phenanthren-3-yl)acetamide (15d): Prepared by using the general procedure above (method A) from 2-([1,1'-binaphthalen]-2-yl)-2-methoxyacetonitrile (14d) $(176.5 \mathrm{mg}, 0.546 \mathrm{mmol})$ except for the reaction conditions of amidation (TFAA $\left(231 \mu \mathrm{L}, 1.64 \mathrm{mmol}, 3\right.$ equiv), $80{ }^{\circ} \mathrm{C}$ for $4 \mathrm{~h}$ ). Purification by flash column chromatography $\left(\mathrm{SiO}_{2}\right.$, hexane-EtOAc $\left.=4: 1\right)$ afforded aryl amine $\mathbf{1 5 d}(192.1 \mathrm{mg}$, $0.458 \mathrm{mmol}, 84 \%)$.

Brown solid; M.p. $237-240{ }^{\circ} \mathrm{C} ;{ }^{1} \mathrm{H}$ NMR $\left(500 \mathrm{MHz},\left(\mathrm{CD}_{3}\right)_{2} \mathrm{SO}\right) \delta 11.60(1 \mathrm{H}, \mathrm{s}), 8.29-8.21(3 \mathrm{H}$, $\mathrm{m}), 8.21-8.12(3 \mathrm{H}, \mathrm{m}), 8.09(1 \mathrm{H}, \mathrm{d}, J=8.0 \mathrm{~Hz}), 7.91(1 \mathrm{H}, \mathrm{d}, J=8.1 \mathrm{~Hz}), 7.63(1 \mathrm{H}, \mathrm{t}, J=7.5 \mathrm{~Hz})$, $7.59(1 \mathrm{H}, \mathrm{t}, J=7.5 \mathrm{~Hz}), 7.38-7.32(2 \mathrm{H}, \mathrm{m}), 3.98(3 \mathrm{H}, \mathrm{s}) ;{ }^{13} \mathrm{C} \mathrm{NMR}\left(125 \mathrm{MHz},\left(\mathrm{CD}_{3}\right)_{2} \mathrm{SO}\right) \delta$ $156.45\left[\mathrm{q},{ }^{2} J(\mathrm{C}, \mathrm{F})=35.8 \mathrm{~Hz}\right], 150.96,132.36,131.57,129.89,129.79,129.10,128.60,128.37$, $128.19,128.13,128.02,127.23,127.15,126.68,126.60,125.07,125.02,123.60,120.73,120.14$, $119.71,116.34\left[\mathrm{q},{ }^{1} J(\mathrm{C}, \mathrm{F})=287.3 \mathrm{~Hz}\right], 62.00 ; \mathrm{IR}(\mathrm{ATR}) \vee 3264,1710,1538,1318,1260,1160$, $1054,813,749 \mathrm{~cm}^{-1}$; HRMS (FD) calcd for $\mathrm{C}_{25} \mathrm{H}_{16} \mathrm{~F}_{3} \mathrm{NO}_{2}\left(\mathrm{M}^{+}\right)$: 419.1133, found: 419.1142.

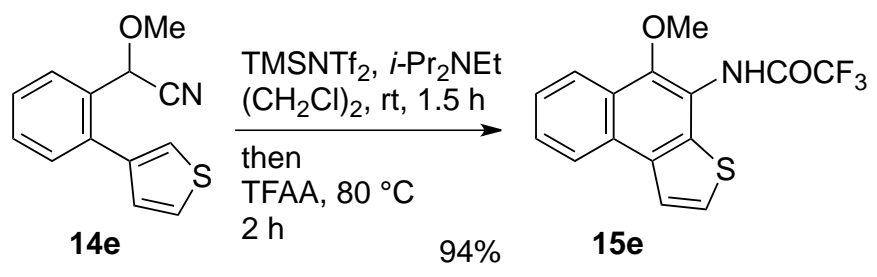

2,2,2-Trifluoro- $N$-(5-methoxynaphtho[2,1-b]thiophen-4-yl)acetamide (15e): Prepared by using the general procedure above (method A) from 2-methoxy-2-(2-(thiophen-3-yl)phenyl)acetonitrile (14e) $(68.8 \mathrm{mg}, 0.300 \mathrm{mmol})$ except for the reaction conditions of amidation (TFAA $(254 \mu \mathrm{L}, 1.80$ mmol, 6 equiv), $80{ }^{\circ} \mathrm{C}$ for $\left.2 \mathrm{~h}\right)$. Purification by flash column chromatography $\left(\mathrm{SiO}_{2}\right.$, hexane-EtOAc $=6: 1)$ afforded aryl amine 15e $(91.4 \mathrm{mg}, 0.281 \mathrm{mmol}, 94 \%)$.

Green solid; M.p. $164-177{ }^{\circ} \mathrm{C} ;{ }^{1} \mathrm{H}$ NMR $\left(500 \mathrm{MHz}, \mathrm{CDCl}_{3}\right) \delta 8.35(1 \mathrm{H}, \mathrm{d}, J=8.0 \mathrm{~Hz}), 8.30-8.04$ $(1 \mathrm{H}, \mathrm{br}-\mathrm{s}), 8.20(1 \mathrm{H}, \mathrm{d}, J=8.6 \mathrm{~Hz}), 7.97(1 \mathrm{H}, \mathrm{d}, J=5.2 \mathrm{~Hz}), 7.71-7.65(1 \mathrm{H}, \mathrm{m}), 7.65-7.57(2 \mathrm{H}$, 
m), 4.01 (3H, s); ${ }^{13} \mathrm{C}$ NMR (125 MHz, CD 3 OD) $\delta 158.11\left[\mathrm{q},{ }^{2} J(\mathrm{C}, \mathrm{F})=37.0 \mathrm{~Hz}\right], 150.96,137.14$, $134.97,130.88,128.62,127.89,126.93,126.72,125.17,124.31,123.50,119.77,117.73$ [q, $\left.{ }^{1} J(C, F)=286.2 \mathrm{~Hz}\right], 62.73$; IR $($ ATR $) \vee 3231,3072,2390,1715,1547,1456,1345,1260,1151$, 1037, $715 \mathrm{~cm}^{-1}$; HRMS (FD) calcd for $\mathrm{C}_{15} \mathrm{H}_{10} \mathrm{~F}_{3} \mathrm{NO}_{2} \mathrm{~S}\left(\mathrm{M}^{+}\right)$: 325.0384, found: 325.0397 .

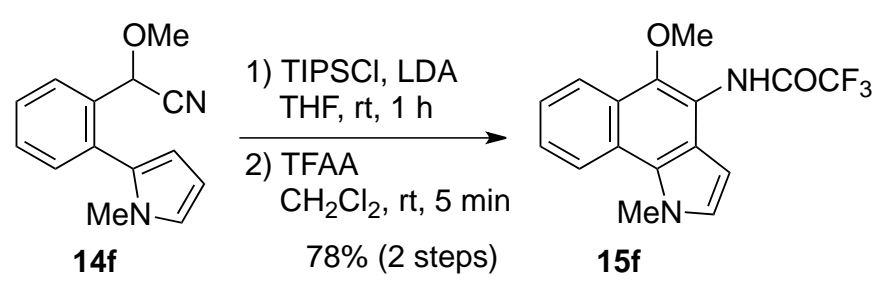

2,2,2-Trifluoro- $N$-(5-methoxy-1-methyl-1H-benzo[g]indol-4-yl)acetamide (15f): Prepared by using the general procedure above (method B) from 2-methoxy-2-(2-(1-methyl-1H-pyrrol-2-yl)phenyl)acetonitrile (14f) $(67.8 \mathrm{mg}, 0.300 \mathrm{mmol})$ except for the reaction conditions of amidation (TFAA $(42.3 \mu \mathrm{L}, 0.300 \mathrm{mmol}, 1$ equiv), room temperature for $5 \mathrm{~min})$. Purification by flash column chromatography $\left(\mathrm{SiO}_{2}\right.$, hexane- $\left.\mathrm{Et}_{2} \mathrm{O}=2: 1\right)$ afforded aryl amine $\mathbf{1 5 f}$ (75.1 $\mathrm{mg}, 0.233 \mathrm{mmol}, 78 \%$ for 2 steps).

White solid; M.p. $127-129{ }^{\circ} \mathrm{C} ;{ }^{1} \mathrm{H}$ NMR $\left(500 \mathrm{MHz}, \mathrm{CDCl}_{3}\right) \delta 8.46(1 \mathrm{H}, \mathrm{d}, J=8.6 \mathrm{~Hz}), 8.30(1 \mathrm{H}$, br-s), $8.20(1 \mathrm{H}, \mathrm{d}, J=8.6 \mathrm{~Hz}), 7.61-7.56(1 \mathrm{H}, \mathrm{m}), 7.54-7.49(1 \mathrm{H}, \mathrm{m}), 7.08(1 \mathrm{H}, \mathrm{d}, J=2.9 \mathrm{~Hz})$, $6.50(1 \mathrm{H}, \mathrm{d}, J=2.9 \mathrm{~Hz}), 4.27(3 \mathrm{H}, \mathrm{s}), 3.93(3 \mathrm{H}, \mathrm{s}) ;{ }^{13} \mathrm{C} \mathrm{NMR}\left(125 \mathrm{MHz}, \mathrm{CD}_{3} \mathrm{OD}\right) \delta 158.07[\mathrm{q}$, $\left.{ }^{2} J(\mathrm{C}, \mathrm{F})=37.0 \mathrm{~Hz}\right], 146.36,131.08,128.73,127.24,126.73,124.90,124.71,124.48,123.75$, 122.10, 118.56, 117.93 [q, ${ }^{1} J(\mathrm{C}, \mathrm{F})=285.0 \mathrm{~Hz}$ ] 101.61, 62.28, 38.60; IR (ATR) v 3246, 2592, 1712, 1446, 1348, 1303, 1153, 1086, 981, 918, 849, 757, 728, 700, $688 \mathrm{~cm}^{-1}$; HRMS (FD) calcd for $\mathrm{C}_{16} \mathrm{H}_{13} \mathrm{~F}_{3} \mathrm{~N}_{2} \mathrm{O}_{2}\left(\mathrm{M}^{+}\right)$: 322.0929, found: 322.1914 .

\section{Synthesis of Steroidal Aryl Amine 19 (Scheme 8)}
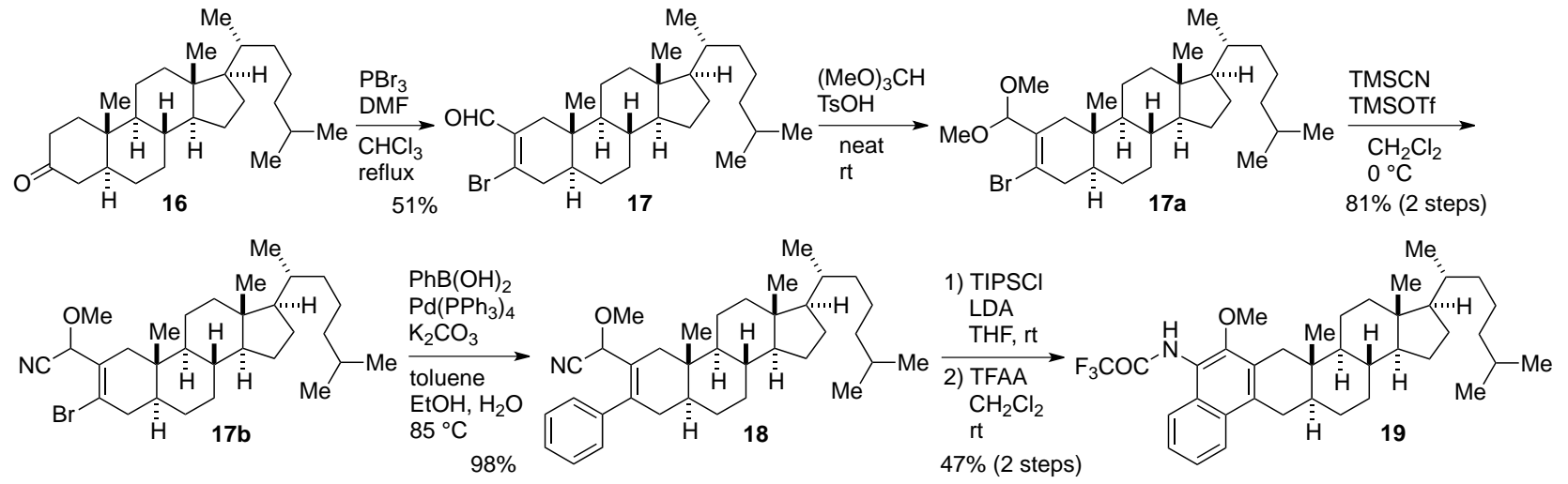

$(5 S, 8 R, 9 S, 10 S, 13 R, 14 S, 17 R)-3-B r o m o-10,13-d i m e t h y l-17-((R)-6-m e t h y l h e p t a n-2-y l)-4,5,6,7,8$, 9,10,11,12,13,14,15,16,17-tetradecahydro- $1 H$-cyclopenta $[a]$ phenanthrene-2-carbaldehyde

(17): Aldehyde 17 was prepared according to the reported procedure. ${ }^{6}$ A mixture of $\mathrm{PBr}_{3}(2.63 \mathrm{~mL}$, 
$28.0 \mathrm{mmol})$ and $\mathrm{DMF}(4.34 \mathrm{~mL}, 56.0 \mathrm{~mL})$ in $\mathrm{CHCl}_{3}(20 \mathrm{~mL})$ was stirred at room temperature for $15 \mathrm{~min}$. Then a solution of ketone $16(2.71 \mathrm{~g}, 7.00 \mathrm{mmol})$ in $\mathrm{CHCl}_{3}(15 \mathrm{~mL})$ was added and the mixture was heated to reflux for $2 \mathrm{~h}$. The reaction mixture was slowly poured into ice-cold water $(200 \mathrm{~mL})$ and basified with saturated aqueous sodium bicarbonate, and the products were extracted with $\mathrm{Et}_{2} \mathrm{O}$. The combined organic layers were dried over $\mathrm{MgSO}_{4}$ and concentrated under reduced pressure. Purification by flash column chromatography $\left(\mathrm{SiO}_{2}\right.$, hexane- $\left.\mathrm{Et}_{2} \mathrm{O}=8: 1\right)$ afforded aldehyde 17 (1.71 g, $3.58 \mathrm{mmol}, 51 \%)$. The spectral data $\left({ }^{1} \mathrm{H}\right.$ NMR) was identical to that was previously reported. ${ }^{6}$

(5S,8R,9S,10S,13R,14S,17R)-3-Bromo-2-(dimethoxymethyl)-10,13-dimethyl-17-( $(R)$-6-methyl heptan-2-yl)-4,5,6,7,8,9,10,11,12,13,14,15,16,17-tetradecahydro- $1 H$-cyclopenta[a]phenanthre ne (17a): To a mixture of aldehyde $17(1.61 \mathrm{~g}, 3.37 \mathrm{mmol})$ and $(\mathrm{MeO})_{3} \mathrm{CH}(11.0 \mathrm{~mL}, 10.1 \mathrm{mmol})$ was added $\mathrm{TsOH} \cdot \mathrm{H}_{2} \mathrm{O}(29.1 \mathrm{mg}, 0.169 \mathrm{mmol})$ and the mixture was stirred at room temperature for $1.5 \mathrm{~h}$. The reaction mixture was diluted with $\mathrm{Et}_{2} \mathrm{O}(50 \mathrm{~mL})$ and washed with saturated aqueous sodium bicarbonate. The organic layer was dried over $\mathrm{MgSO}_{4}$ and concentrated under reduced pressure to give dimethyl acetal 17a $(2.31 \mathrm{~g})$. This material was used for the next step without further purification.

2-((5S,8R,9S,10S,13R,14S,17R)-3-Bromo-10,13-dimethyl-17-(6-methylheptan-2-yl)-4,5,6,7,8,9 ,10,11,12,13,14,15,16,17-tetradecahydro-1H-cyclopenta[a]phenanthren-2-yl)-2-methoxyaceto nitrile (17b): To a solution of the above dimethyl acetal 17a $(2.31 \mathrm{~g}$, as $3.37 \mathrm{mmol})$ and TMSCN $(627 \mu \mathrm{L}, 5.01 \mathrm{mmol})$ in $\mathrm{CH}_{2} \mathrm{Cl}_{2}(17 \mathrm{~mL})$ was added TMSOTf $(61 \mu \mathrm{L}, 0.337 \mathrm{mmol})$ and the mixture was stirred at $0{ }^{\circ} \mathrm{C}$ for $1 \mathrm{~h}$. Saturated aqueous sodium bicarbonate $(10 \mathrm{~mL})$ was added and the products were extracted with $\mathrm{Et}_{2} \mathrm{O}$. The combined organic layers were dried over $\mathrm{MgSO}_{4}$ and concentrated under reduced pressure. The residue was purified by flash column chromatography $\left(\mathrm{SiO}_{2}\right.$, hexane-Et $\left.2 \mathrm{O}=10: 1\right)$ to give cyanohydrin methyl ether $\mathbf{1 7 b}(1.41 \mathrm{~g}, 2.72 \mathrm{mmol}, 81 \%$ for 2 steps) as a 67:33 mixture of diastereomers.

Colorless oil; $[\alpha]_{\mathrm{D}}{ }^{23} 57.7$ (c 1.12, $\left.\mathrm{CHCl}_{3}\right) ;{ }^{1} \mathrm{H}$ NMR $\left(500 \mathrm{MHz}, \mathrm{CDCl}_{3}\right) \delta 5.20(1 \mathrm{H}, \mathrm{s}), 3.47(0.7 \mathrm{H}$, s), $3.44(0.3 \mathrm{H}, \mathrm{s}), 2.47-2.19(3 \mathrm{H}, \mathrm{m}), 2.04-1.64(5 \mathrm{H}, \mathrm{m}), 1.60-0.93(21 \mathrm{H}, \mathrm{m}), 0.90(3 \mathrm{H}, \mathrm{d}, J=6.3$ $\mathrm{Hz}), 0.98-0.94(6 \mathrm{H}, \mathrm{m}), 0.81-0.70(4 \mathrm{H}, \mathrm{m}), 0.68-0.64(4 \mathrm{H}, \mathrm{m}) ;{ }^{13} \mathrm{C} \mathrm{NMR}\left(125 \mathrm{MHz}, \mathrm{CDCl}_{3}\right) \delta$ 128.42 , 128.22, 124.93, 123.89, 116.36, 116.24, 73.98, 72.39, 57.68, 57.41, 56.23, 56.20, 56.15, $56.13,53.35,53.24,43.55,43.31,42.41,41.30,41.26,40.83,40.15,39.71,39.68,39.48,36.12$, $35.75,35.73,35.38,35.30,34.79,34.54,31.34,31.31,28.14,27.96,27.72,27.67,24.16,23.79$, 22.80, 22.54, 21.10, 18.65, 11.95, 11.78, 11.42; IR (ATR) v 2929, 2324, 1661, 1465, 1383, 1337, 1184, 1092, $959 \mathrm{~cm}^{-1}$; HRMS (FD) calcd for $\mathrm{C}_{30} \mathrm{H}_{48} \mathrm{BrNO}\left(\mathrm{M}^{+}\right)$: 517.2919, found: 517.2938.

2-((5S,8R,9S,10S,13R,14S,17R)-10,13-Dimethyl-17-(6-methylheptan-2-yl)-3-phenyl-4,5,6,7,8,9

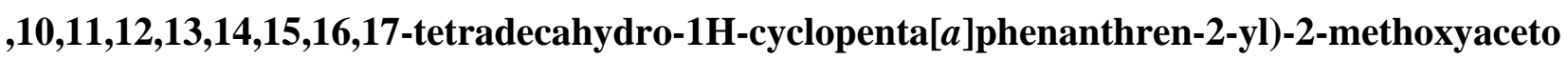


nitrile (18): To a mixture of cyanohydrin methyl ether $\mathbf{1 7 b}(1.02 \mathrm{~g}, 1.97 \mathrm{mmol}), \mathrm{PhB}(\mathrm{OH})_{2}(263.4$ $\mathrm{mg}, 2.16 \mathrm{mmol})$, and $\mathrm{K}_{2} \mathrm{CO}_{3}(544.5 \mathrm{mg}, 3.94 \mathrm{mmol})$ in toluene- $\mathrm{EtOH}-\mathrm{H}_{2} \mathrm{O}(3: 3: 1,6.2 \mathrm{~mL})$ was added $\mathrm{Pd}\left(\mathrm{PPh}_{3}\right)_{4}(68.3 \mathrm{mg}, 0.0591 \mathrm{mmol})$ and the reaction mixture was stirred at $85{ }^{\circ} \mathrm{C}$ for $10 \mathrm{~h}$. The mixture was filtered through a pad of Celite. To the filtrate was added water $(3 \mathrm{~mL})$ and the products were extracted with $\mathrm{Et}_{2} \mathrm{O}$. The combined organic layers were dried over $\mathrm{MgSO}_{4}$ and concentrated under reduced pressure. Purification by flash column chromatography $\left(\mathrm{SiO}_{2}\right.$, hexane- $\left.\mathrm{Et}_{2} \mathrm{O}=10: 1\right)$ afforded nitrile $\mathbf{1 8}(991.2 \mathrm{mg}, 1.92 \mathrm{mmol}, 98 \%)$ as a 68:32 mixture of diastereomers.

White solid; M.p. ${ }^{106}-110{ }^{\circ} \mathrm{C}$; $[\alpha]_{\mathrm{D}}{ }^{22} 90.5$ (c 1.10, $\mathrm{CHCl}_{3}$ ); ${ }^{1} \mathrm{H}$ NMR $\left(500 \mathrm{MHz}, \mathrm{CDCl}_{3}\right) \delta$ 7.39-7.27 (3H, m), $7.12(2 \mathrm{H}, \mathrm{d}, J=6.9 \mathrm{~Hz}), 4.57(0.7 \mathrm{H}, \mathrm{s}), 4.52(0.3 \mathrm{H}, \mathrm{s}), 3.30-3.27(3 \mathrm{H}, \mathrm{m})$, 2.40-2.17 (2H, m), 2.10-1.74 (4H, m), 1.73-1.67 (1H, m), 1.64-0.95 (20H, m), 0.95-0.75 (14H, $\mathrm{m}), 0.70-0.66(3 \mathrm{H}, \mathrm{m}) ;{ }^{13} \mathrm{C} \mathrm{NMR}\left(125 \mathrm{MHz}, \mathrm{CDCl}_{3}\right) \delta 141.14,140.96,140.78,140.76,140.57$, $128.67,128.56,128.45,127.60,127.57,127.48,127.43,127.07,125.71,117.49,117.39,71.83$, $70.24,57.10,56.79,56.38,56.32,56.21,56.16,53.79,53.67,42.43,41.65,41.35,39.84,39.82$, $39.48,38.73,37.95,37.54,37.36,36.14,35.77,35.75,35.56,35.48,34.54,34.29,31.55,28.16$, $28.11,28.05,27.95,24.17,23.80,22.78,22.54,21.17,18.67,11.98,11.71,11.37$; IR (ATR) v 2929, 2153, 1442, 1382, 1219, 1089, 773, $701 \mathrm{~cm}^{-1}$; HRMS (FD) calcd for $\mathrm{C}_{36} \mathrm{H}_{53} \mathrm{NO}\left(\mathrm{M}^{+}\right)$: 515.4127, found: 515.4129 .

\section{2,2,2-Trifluoro- $N$-((1R,3a $S, 3 \mathrm{~b} R, 5 \mathrm{a} S, 13 \mathrm{a} S, 13 \mathrm{~b} S, 15 \mathrm{a} R)$-12-methoxy-13a,15a-dimethyl-1-(6-met hylheptan-2-yl)-2,3,3a,3b,4,5,5a,6,13,13a,13b,14,15,15a-tetradecahydro-1H-benzo[ $k]$ cyclopen} ta[c]tetraphen-11-yl)acetamide (19): Prepared by using the general procedure above (method B) from nitrile $18(51.6 \mathrm{mg}, 0.100 \mathrm{mmol})$ except for the reaction time of cyclization $(2 \mathrm{~h})$ and the reaction time of amidation $(2 \mathrm{~h})$. Purification by flash column chromatography $\left(\mathrm{SiO}_{2}\right.$, hexane- $\left.\mathrm{Et}_{2} \mathrm{O}=10: 1\right)$ afforded aryl amine $19(28.7 \mathrm{mg}, 0.0469 \mathrm{mmol}, 47 \%)$.

Colorless oil; $[\alpha]_{\mathrm{D}}{ }^{23} 3.04$ (c 7.34, $\left.\mathrm{CHCl}_{3}\right) ;{ }^{1} \mathrm{H} \mathrm{NMR}\left(500 \mathrm{MHz}, \mathrm{CDCl}_{3}\right) \delta 7.98(1 \mathrm{H}, \mathrm{d}, J=8.6 \mathrm{~Hz})$, $7.95(1 \mathrm{H}, \mathrm{s}), 7.65(1 \mathrm{H}, \mathrm{d}, J=7.5 \mathrm{~Hz}), 7.53-7.44(2 \mathrm{H}, \mathrm{m}), 3.78(3 \mathrm{H}, \mathrm{s}), 3.10(1 \mathrm{H}, \mathrm{dd}, J=17.2,4.9$ $\mathrm{Hz}), 3.01(1 \mathrm{H}, \mathrm{d}, J=16.6 \mathrm{~Hz}), 2.71-2.59(1 \mathrm{H}, \mathrm{m}), 2.26(1 \mathrm{H}, \mathrm{d}, J=16.6 \mathrm{~Hz}), 2.07(1 \mathrm{H}, \mathrm{d}, J=12.6$ $\mathrm{Hz}), 1.91-1.75(2 \mathrm{H}, \mathrm{m}), 1.74-0.97(21 \mathrm{H}, \mathrm{m}), 0.94(3 \mathrm{H}, \mathrm{d}, J=6.3 \mathrm{~Hz}), 0.91-0.84(7 \mathrm{H}, \mathrm{m}), 0.70$ $(3 \mathrm{H}, \mathrm{s}), 0.68(3 \mathrm{H}, \mathrm{s}) ;{ }^{13} \mathrm{C} \mathrm{NMR}\left(125 \mathrm{MHz}, \mathrm{CDCl}_{3}\right) \delta 156.84\left[\mathrm{q},{ }^{2} J(\mathrm{C}, \mathrm{F})=35.8 \mathrm{~Hz}\right], 152.66,134.38$, $129.62,129.33,128.22,126.25,125.47,123.53,122.22,118.89,116.19$ [q, ${ }^{1} J(C, F)=287.4 \mathrm{~Hz}$ ], $60.81,56.44,56.32,53.79,42.48,41.17,39.98,39.50,38.14,36.17,35.79,35.60,34.22,31.65$, $31.09,28.84,28.22,28.00,24.23,23.85,22.81,22.56,21.18,18.69,12.00,11.35$; IR (ATR) v 3276, 2932, 1728, 1524, 1456, 1383, 1316, 1212, 1168, 1028, 944, $755 \mathrm{~cm}^{-1}$; HRMS (FD) calcd for $\mathrm{C}_{38} \mathrm{H}_{52} \mathrm{~F}_{3} \mathrm{NO}_{2}\left(\mathrm{M}^{+}\right)$: 611.3950 , found: 611.3952 . 
Synthesis of Aryl Amine 22 (Reference 11)

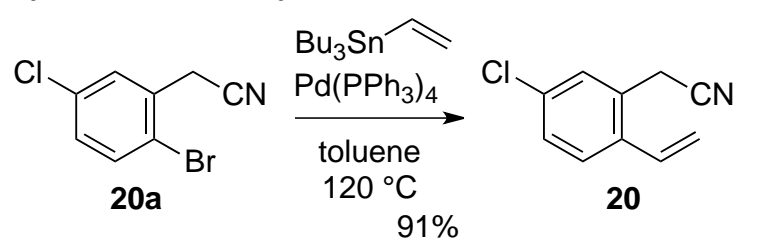

2-(5-Chloro-2-vinylphenyl)acetonitrile (20): To a mixture of aryl bromide 20a (491.5 mg, 2.13 mmol) ${ }^{6}$ and tributyl(vinyl)stannane $(748 \mu \mathrm{L}, 2.56 \mathrm{mmol})$ in toluene $(5.3 \mathrm{~mL})$ was added $\mathrm{Pd}\left(\mathrm{PPh}_{3}\right)_{4}$ (98.4 mg, $0.0852 \mathrm{mmol}$ ) and the mixture was stirred at $120^{\circ} \mathrm{C}$ for $3 \mathrm{~h}$. The reaction mixture was filtered through a pad of Celite and the filtrate was concentrated under reduced pressure. Purification by flash column chromatography $\left(10 \mathrm{wt} \% \mathrm{~K}_{2} \mathrm{CO}_{3} / \mathrm{SiO}_{2}\right.$, hexane- $\left.\mathrm{Et}_{2} \mathrm{O}=4: 1\right)$ afforded nitrile 20 (345.8 mg, $1.95 \mathrm{mmol}, 91 \%$ ).

Colorless oil; ${ }^{1} \mathrm{H}$ NMR (500 MHz, $\left.\mathrm{CDCl}_{3}\right) \delta 7.49(1 \mathrm{H}, \mathrm{d}, J=8.0 \mathrm{~Hz}), 7.40(1 \mathrm{H}, \mathrm{d}, J=1.8 \mathrm{~Hz})$, $7.31(1 \mathrm{H}, \mathrm{dd}, J=8.6,1.8 \mathrm{~Hz}), 6.78(1 \mathrm{H}, \mathrm{dd}, J=17.2,10.9 \mathrm{~Hz}), 5.69(1 \mathrm{H}, \mathrm{d}, J=17.2 \mathrm{~Hz}), 5.47$ $(1 \mathrm{H}, \quad \mathrm{d}, \quad J=11.5 \mathrm{~Hz}), \quad 3.73 \quad(2 \mathrm{H}, \quad \mathrm{s}) ; \quad{ }^{13} \mathrm{C} \quad \mathrm{NMR} \quad\left(125 \quad \mathrm{MHz}, \quad \mathrm{CD}_{3} \mathrm{OD}\right)$ $\delta 136.92,134.87,133.36,131.45,129.92,129.61,128.97,118.73,118.69,21.51 ; \quad$ IR $(\mathrm{ATR}) \quad v$ 2933, 2363, 2253, 1734, 1594, 1482, 1415, 1220, 1117, 984, 924, 865, 827, $882 \mathrm{~cm}^{-1}$; HRMS (FD) calcd for $\mathrm{C}_{10} \mathrm{H}_{8} \mathrm{ClN}\left(\mathrm{M}^{+}\right)$: 177.0345, found: 177.0339 .

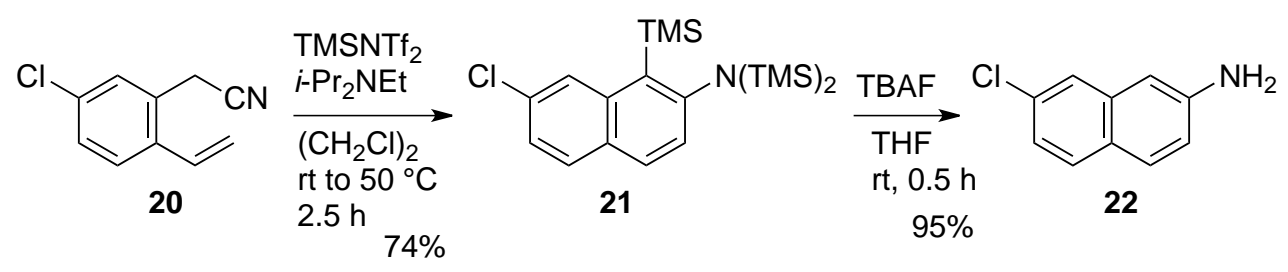

\section{$N$-(7-Chloro-1-(trimethylsilyl)naphthalen-2-yl)-1,1,1-trimethyl- $N$-(trimethylsilyl)silanamine}

(21): To a mixture of nitrile $20(22.4 \mathrm{mg}, 0.126 \mathrm{mmol})$ and $i$ - $\operatorname{Pr}_{2} \mathrm{NEt}(82 \mu \mathrm{L}, 0.504 \mathrm{mmol})$ in $\left(\mathrm{CH}_{2} \mathrm{Cl}\right)_{2}(0.63 \mathrm{~mL})$ was added TMSNTf $2(116 \mu \mathrm{L}, 0.504 \mathrm{mmol})$ and the reaction mixture was stirred at room temperature for $1 \mathrm{~h}$ and then stirred at $50{ }^{\circ} \mathrm{C}$ for $1.5 \mathrm{~h}$. Saturated aqueous sodium bicarbonate $(0.1 \mathrm{~mL})$ was added to the mixture and the products were extracted with $\mathrm{Et}_{2} \mathrm{O}$. The combined organic layers were dried over $\mathrm{MgSO}_{4}$ and concentrated under reduced pressure. Purification by flash column chromatography $\left(\mathrm{SiO}_{2}\right.$, hexane- $\left.\mathrm{Et}_{2} \mathrm{O}=15: 1\right)$ afforded silyl amine 21 (36.7 mg, $0.0931 \mathrm{mmol}, 74 \%$ ).

Colorless oil; ${ }^{1} \mathrm{H}$ NMR $\left(500 \mathrm{MHz}, \mathrm{CDCl}_{3}\right) \delta 8.15(1 \mathrm{H}, \mathrm{s}), 7.71(1 \mathrm{H}, \mathrm{d}, J=9.2 \mathrm{~Hz}), 7.66(1 \mathrm{H}, \mathrm{d}, J$ $=8.6 \mathrm{~Hz}), 7.32(1 \mathrm{H}, \mathrm{d}, J=8.6 \mathrm{~Hz}), 7.07(1 \mathrm{H}, \mathrm{d}, J=8.6 \mathrm{~Hz}), 0.53(9 \mathrm{H}, \mathrm{s}), 0.10(18 \mathrm{H}, \mathrm{s}) ;{ }^{13} \mathrm{C} \mathrm{NMR}$ $\left(125 \mathrm{MHz} \mathrm{CDCl}_{3}\right) \delta 152.93,138.98,133.84,131.15,130.91,130.07,129.59,129.40,127.24$, 124.66, 4.15, 2.30; IR (ATR) v 2956, 1608, 1495, 1341, 1249, 1206, 1171, 1143, 1091, 1001, 928, 819, 754, $668 \mathrm{~cm}^{-1}$; HRMS (FD) calcd for $\mathrm{C}_{19} \mathrm{H}_{32} \mathrm{ClNSi}_{3}\left(\mathrm{M}^{+}\right): 393.1531$, found: 393.1543 .

7-Chloronaphthalen-2-amine (22): To a solution of silyl amine 21 (36.7 $\mathrm{mg}, 0.0931 \mathrm{mmol})$ in 
THF (0.47 mL) was added TBAF (1.0 M in THF, $279 \mu \mathrm{L}, 0.279 \mathrm{mmol})$, and the reaction mixture was stirred at room temperature for $30 \mathrm{~min} . \mathrm{H}_{2} \mathrm{O}(0.5 \mathrm{~mL})$ was added to the mixture and the products were extracted with $\mathrm{Et}_{2} \mathrm{O}$. The combined organic layers were dried over $\mathrm{MgSO}_{4}$ and concentrated under reduced pressure. The residue was purified by flash column chromatography $\left(\mathrm{SiO}_{2}\right.$, hexane-EtOAc $\left.=4: 1\right)$ afforded free amine $22(15.7 \mathrm{mg}, 0.0884 \mathrm{mmol}, 95 \%)$.

White solid; M.p. $116-120{ }^{\circ} \mathrm{C} ;{ }^{1} \mathrm{H}$ NMR $\left(500 \mathrm{MHz}, \mathrm{CDCl}_{3}\right) \delta 7.62(1 \mathrm{H}, \mathrm{d}, J=8.0 \mathrm{~Hz}), 7.61(1 \mathrm{H}$, $\mathrm{d}, J=8.1 \mathrm{~Hz}), 7.56(1 \mathrm{H}, \mathrm{d}, J=1.7 \mathrm{~Hz}), 7.15(1 \mathrm{H}, \mathrm{dd}, J=8.6,2.3 \mathrm{~Hz}), 6.92(1 \mathrm{H}, \mathrm{dd}, J=8.6,2.3$ $\mathrm{Hz}), 6.88(1 \mathrm{H}, \mathrm{d}, J=1.7 \mathrm{~Hz}), 3.91(2 \mathrm{H}, \mathrm{br}-\mathrm{s}) ;{ }^{13} \mathrm{C} \mathrm{NMR}\left(125 \mathrm{MHz}, \mathrm{CDCl}_{3}\right) \delta 145.04,135.65$, 132.12, 129.23, 129.14, 126.03, 124.39, 123.18, 118.29, 107.46; IR (ATR) v 3445, 3335, 2925, 1631, 1505, 1387, 1257, 1073, 892, $831 \mathrm{~cm}^{-1}$; HRMS (FD) calcd for $\mathrm{C}_{10} \mathrm{H}_{8} \mathrm{ClN}\left(\mathrm{M}^{+}\right)$: 177.0345 , found: 177.0337 .

\section{Synthesis of Hydroxy Naphthylamine Derivative (Scheme 6)}

$o$-Vinylbenzaldehyde ${ }^{2}$ was directly converted into hydroxy aryl amine $\mathbf{1 3 \mathbf { j }}$ in $61 \%$ by the sequential treatment with TMSCN, TMSNTf 2 , TFAA, and saturated aqueous $\mathrm{NaHCO}_{3}$ in one-pot, where cyanation-electrocyclization-amidation-deprotection of the resulting $O$-trifluoroacetate all occurred.

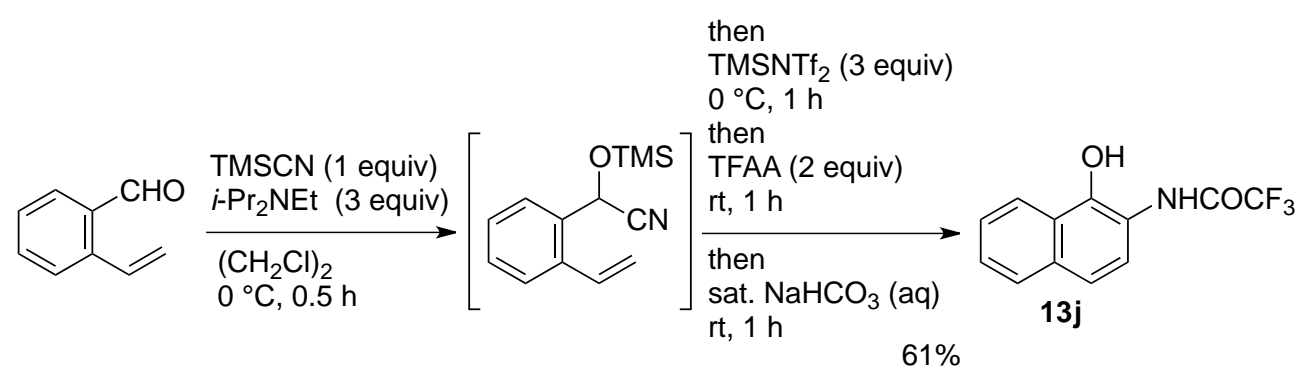

2,2,2-Trifluoro- $N$-(1-hydroxynaphthalen-2-yl)acetamide $\quad(\mathbf{1 3 j}): \quad$ To a mixture of $o$-vilylbenzaldehyde $(52.9 \mathrm{mg}, 0.400 \mathrm{mmol})^{2}$ and $i$ - $\operatorname{Pr}_{2} \mathrm{NEt}(196 \mu \mathrm{L}, 1.20 \mathrm{mmol}, 3$ equiv) in $\left(\mathrm{CH}_{2} \mathrm{Cl}\right)_{2}(2.00 \mathrm{~mL})$ was added TMSCN $\left(50 \mu \mathrm{L}, 0.400 \mathrm{mmol}, 1\right.$ equiv) at $0{ }^{\circ} \mathrm{C}$, and the reaction mixture was stirred at this temperature for $30 \mathrm{~min}$. To the mixture was added $\mathrm{TMSNTf}_{2}(275 \mu \mathrm{L}$, $1.20 \mathrm{mmol}, 3$ equiv), and the resulting mixture was stirred at $0{ }^{\circ} \mathrm{C}$ for $1 \mathrm{~h}$. TFAA $(113 \mu \mathrm{L}, 0.800$ mmol, 2 equiv) was then added to the reaction mixture, and the mixture was stirred at room temperature for $1 \mathrm{~h}$. The reaction mixture was basified by slow addition of $\mathrm{NaHCO}_{3}(\mathrm{pH}$ of organic layer was ca. 8 , as monitored by $\mathrm{pH}$ paper), and the mixture was vigorously stirred at room temperature for $1 \mathrm{~h}$. The resultant $O$-trifluoroacetate was hydrolyzed at this stage. The products were extracted with $\mathrm{Et}_{2} \mathrm{O}(1 \mathrm{~mL} \times 3)$, dried over $\mathrm{MgSO}_{4}$, and concentrated under reduced pressure. Purification by flash column chromatography [Merck KGaA, Silica Gel 60 (particle size $0.040-0.063 \mathrm{~mm})$, hexane-EtOAc $=6: 1]$ afforded hydroxy naphthylamine derivative $\mathbf{1 3 j}$ (62.7 $\mathrm{mg}$, $0.246 \mathrm{mmol}, 61 \%)$.

Gray solid; M.p. $140-143{ }^{\circ} \mathrm{C} ;{ }^{1} \mathrm{H}$ NMR $\left(500 \mathrm{MHz}, \mathrm{CDCl}_{3}\right) \delta 8.35(1 \mathrm{H}, \mathrm{br}-\mathrm{s}), 8.29(1 \mathrm{H}, \mathrm{d}, J=7.4$ 
$\mathrm{Hz}), 7.83-7.77(1 \mathrm{H}, \mathrm{m}), 7.59-7.50(3 \mathrm{H}, \mathrm{m}), 7.48(1 \mathrm{H}, \mathrm{d}, J=9.2 \mathrm{~Hz}), 7.34(1 \mathrm{H}, \mathrm{d}, J=8.6 \mathrm{~Hz}) ;{ }^{13} \mathrm{C}$ NMR $\left(125 \mathrm{MHz}, \mathrm{CD}_{3} \mathrm{CN}\right) \delta 157.03\left[\mathrm{q},{ }^{2} J(\mathrm{C}, \mathrm{F})=40.0 \mathrm{~Hz}\right], 146.19,134.28,128.45,127.66$, 126.80, 126.53, 124.02, 122.93, 121.27, 117.65, 116.85 [q, ${ }^{1} J(\mathrm{C}, \mathrm{F})=285.0 \mathrm{~Hz}$ ]; IR (ATR) v 3395, 1702, 1606, 1544, 1516, 1357, 1267, 1152, 950, 900, 801, $647 \mathrm{~cm}^{-1}$; HRMS (FD) calcd for $\mathrm{C}_{12} \mathrm{H}_{8} \mathrm{~F}_{3} \mathrm{NO}_{2}\left(\mathrm{M}^{+}\right): 255.0507$, found: 255.0502.

\section{Synthesis of Aryl Amines 10a and 10b (Table S2)}<smiles>C=Cc1ccccc1C(C)OC</smiles>

9

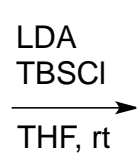

$46 \%$<smiles>COc1c(NCc2ccccc2)ccc2ccccc12</smiles>

$10 \mathrm{a}$

1-tert-Butyl- $N$-(1-methoxynaphthalen-2-yl)-1,1-dimethylsilanamine (10a): To a solution of $i$ - $\operatorname{Pr}_{2} \mathrm{NH}(49 \mu \mathrm{L}, 0.348 \mathrm{mmol}, 1.16$ equiv) in THF (1.5 mL) was added $n$-BuLi (2.65 M in hexane, $125 \mu \mathrm{L}, 0.330 \mathrm{mmol}, 1.10$ equiv) at $-78^{\circ} \mathrm{C}$ and the mixture was stirred at this temperature for 15 min. To the mixture was added nitrile $9\left(52.0 \mathrm{mg}, 50 \mu \mathrm{L}, 0.30 \mathrm{mmol}, 1\right.$ equiv) at $-78{ }^{\circ} \mathrm{C}$, and the resulting mixture was stirred at this temperature for $1 \mathrm{~h}$. TBSCl $(64 \mu \mathrm{L}, 0.300 \mathrm{mmol}, 1$ equiv) was then added, and the mixture was stirred at $-78{ }^{\circ} \mathrm{C}$ for $5 \mathrm{~min}$. The mixture was warmed to room temperature and stirred at this temperature for $1 \mathrm{~h}$. The reaction was quenched by addition of saturated aqueous $\mathrm{NH}_{4} \mathrm{Cl}(3 \mathrm{~mL})$, and the resulting mixture was extracted with $\mathrm{Et}_{2} \mathrm{O}(2 \mathrm{~mL} \times 2)$. The combined organic extracts were dried over $\mathrm{MgSO}_{4}$ and concentrated under reduced pressure. Purification by flash column chromatography $\left(\mathrm{SiO}_{2}\right.$, hexane-EtOAc $\left.=20: 1\right)$ afforded silyl amine $10 a(39.7 \mathrm{mg}, 0.138 \mathrm{mmol}, 46 \%)$.

Yellow oil; ${ }^{1} \mathrm{H}$ NMR $\left(500 \mathrm{MHz}, \mathrm{CDCl}_{3}\right) \delta 7.87(1 \mathrm{H}, \mathrm{d}, J=8.6 \mathrm{~Hz}), 7.70(1 \mathrm{H}, \mathrm{d}, J=8.6 \mathrm{~Hz}), 7.45$ $(1 \mathrm{H}, \mathrm{d}, J=9.2 \mathrm{~Hz}), 7.41(1 \mathrm{H}, \mathrm{t}, J=7.5 \mathrm{~Hz}), 7.21(1 \mathrm{H}, \mathrm{t}, J=7.5 \mathrm{~Hz}), 7.16(1 \mathrm{H}, \mathrm{d}, J=9.2 \mathrm{~Hz}), 4.23$ $(1 \mathrm{H}, \mathrm{s}), 3.86(3 \mathrm{H}, \mathrm{s}), 1.01(9 \mathrm{H}, \mathrm{s}), 0.31(6 \mathrm{H}, \mathrm{s}) ;{ }^{13} \mathrm{C} \mathrm{NMR}\left(125 \mathrm{MHz}, \mathrm{CDCl}_{3}\right) \delta 140.27,137.23$, $128.21,127.98,127.93,126.07,124.39,122.04,119.87,118.38,59.78,26.36,17.96,-3.82$; IR $\left(\right.$ ATR) $v$ 3391, 2928, 2856, 1599, 1477, 1390, 1295, 1079, 981, $828 \mathrm{~cm}^{-1}$; HRMS (FD) calcd for $\mathrm{C}_{17} \mathrm{H}_{25} \mathrm{NOSi}\left(\mathrm{M}^{+}\right)$: 287.1705, found: 287.1711 .

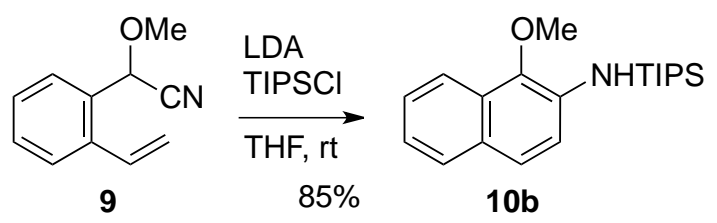

1,1,1-Triisopropyl- $N$-(1-methoxynaphthalen-2-yl)silanamine (10b): To a solution of $i$ - $\operatorname{Pr}_{2} \mathrm{NH}$ (49 $\mu \mathrm{L}, 0.348 \mathrm{mmol}, 1.16$ equiv) in THF (1.5 mL) was added $n$-BuLi $(2.65 \mathrm{M}$ in hexane, $125 \mu \mathrm{L}$, $0.330 \mathrm{mmol}, 1.10$ equiv) at $-78{ }^{\circ} \mathrm{C}$ and the mixture was stirred at this temperature for $15 \mathrm{~min}$. Nitrile 9 (52.0 mg, $50 \mu \mathrm{L}, 0.30 \mathrm{mmol}, 1$ equiv) was then added, and the mixture was stirred at 
$-78{ }^{\circ} \mathrm{C}$ for $1 \mathrm{~h}$. TIPSCl $(64 \mu \mathrm{L}, 0.300 \mathrm{mmol}, 1$ equiv) was then added, and the resulting mixture was stirred at $-78{ }^{\circ} \mathrm{C}$ for $5 \mathrm{~min}$. The mixture was warmed to room temperature and stirred at this temperature for $1 \mathrm{~h}$. The reaction was quenched by addition of saturated aqueous $\mathrm{NH}_{4} \mathrm{Cl}(3 \mathrm{~mL})$, and the resulting mixture was extracted with $\mathrm{Et}_{2} \mathrm{O}(2 \mathrm{~mL} \times 2)$. The combined organic extracts were dried over $\mathrm{MgSO}_{4}$ and concentrated under reduced pressure. Purification by flash column chromatography $\left(\mathrm{SiO}_{2}\right.$, hexane-EtOAc $\left.=10: 1\right)$ afforded silyl amine 10b $(83.9 \mathrm{mg}, 0.255 \mathrm{mmol}$, $85 \%)$.

Yellow oil; ${ }^{1} \mathrm{H}$ NMR $\left(500 \mathrm{MHz}, \mathrm{CDCl}_{3}\right) \delta 7.87(1 \mathrm{H}, \mathrm{d}, J=8.6 \mathrm{~Hz}), 7.69(1 \mathrm{H}, \mathrm{d}, J=8.0 \mathrm{~Hz}), 7.45$ $(1 \mathrm{H}, \mathrm{d}, J=9.2 \mathrm{~Hz}), 7.41(1 \mathrm{H}, \mathrm{t}, J=7.8 \mathrm{~Hz}), 7.20(1 \mathrm{H}, \mathrm{t}, J=7.5 \mathrm{~Hz}), 7.11(1 \mathrm{H}, \mathrm{d}, J=9.2 \mathrm{~Hz}), 4.27$ $(1 \mathrm{H}, \mathrm{s}), 3.88(3 \mathrm{H}, \mathrm{s}), 1.37(3 \mathrm{H}, \mathrm{sept}, J=7.5 \mathrm{~Hz}), 1.14(18 \mathrm{H}, \mathrm{d}, J=7.5 \mathrm{~Hz}) ;{ }^{13} \mathrm{C} \mathrm{NMR}(125 \mathrm{MHz}$, $\left.\mathrm{CDCl}_{3}\right) \delta 139.95,137.49,128.22,127.94,127.86,126.07,124.43,121.90,119.81,118.54,59.63$, 18.49, 12.61; IR (ATR) v 3383, 2941, 2865, 1598, 1477, 1399, 1298, 1080, 981, 880, $801 \mathrm{~cm}^{-1}$; HRMS (FD) calcd for $\mathrm{C}_{20} \mathrm{H}_{31} \mathrm{NOSi}\left(\mathrm{M}^{+}\right)$: 329.2175 , found: 329.2154 .

\section{References}

(1) Harrowven, D. C.; Curran, D. P.; Kostiuk, S. L.; Wallis-Guy, I. L.; Whiting, S.; Stenning, K. J.; Tang, B.; Packard, E.; Nanson, L. Chem. Commun. 2010, 46, 6335-6337.

(2) Jagdale, A. R.; Youn, S. W. Eur. J. Org. Chem. 2011, 3904-3910.

(3) Scheiper, B.; Bonnekessel, M.; Krause, H.; Fürstner, A. J. Org. Chem. 2004, 69, 3943-3949.

(4) Cacchi, S.; Morera, E.; Ortar, G. Tetrahedron Lett. 1984, 25, 2271-2274.

(5) Burger, M. T.; Han, W.; Lan, J.; Nishiguchi, G. U.S. Patent US2010/0056576 A1, 2010.

(6) Gogoi, S.; Shekarrao, K.; Duarah, A.; Bora, T. C.; Gogoi, S.; Boruah, R. C. Steroids 2012, 77, 1438-1445.

(7) Hsieh, J.; Cheng, A.; Fu, J.; Kang, T. Org. Biol. Chem. 2012, 10, 6404-6409. 


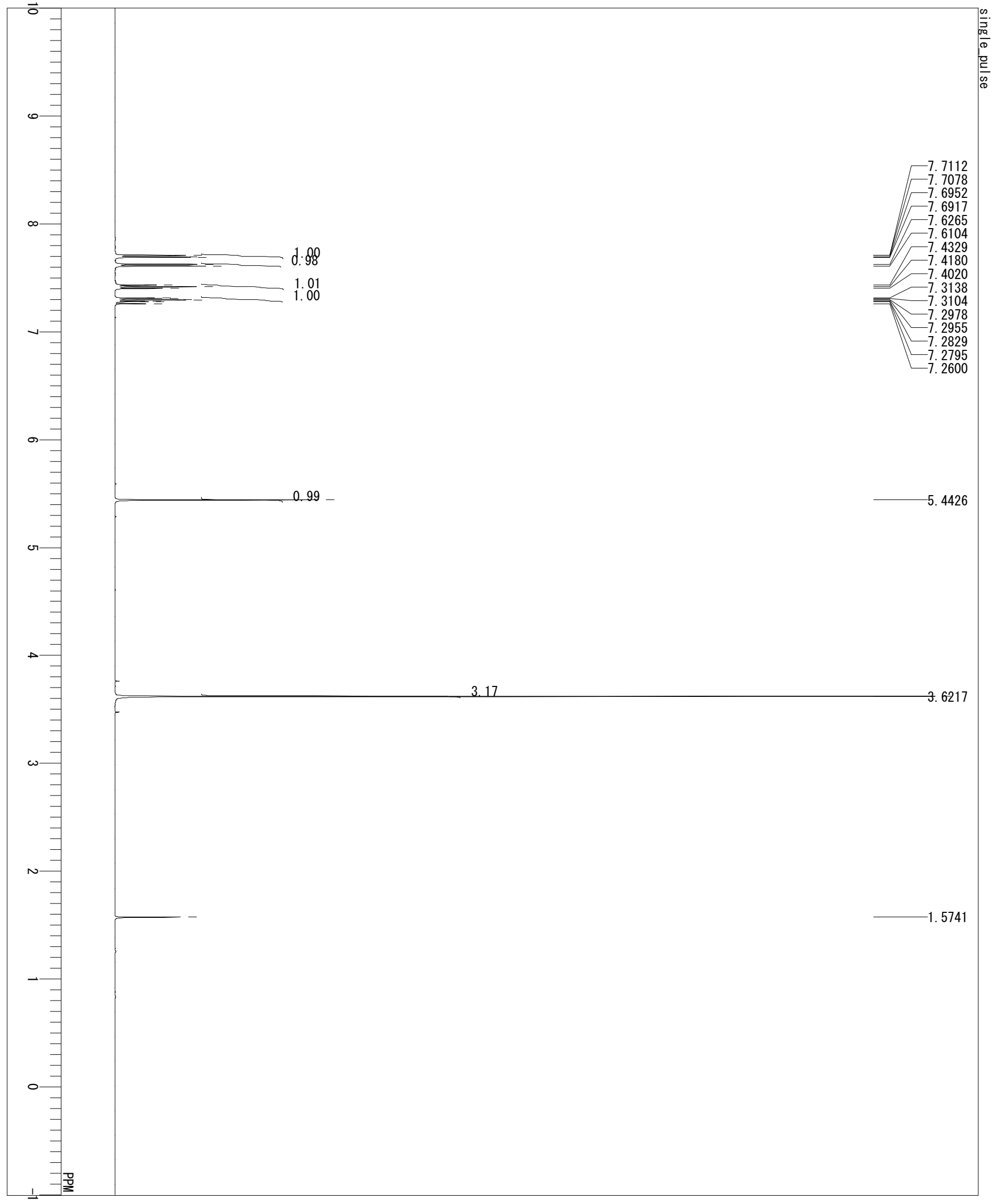

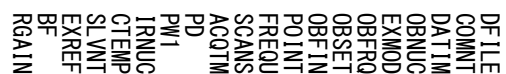
$\stackrel{8}{\stackrel{8}{\omega}} \equiv$

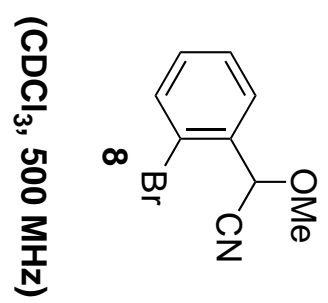

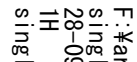

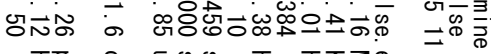

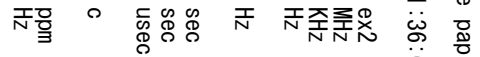




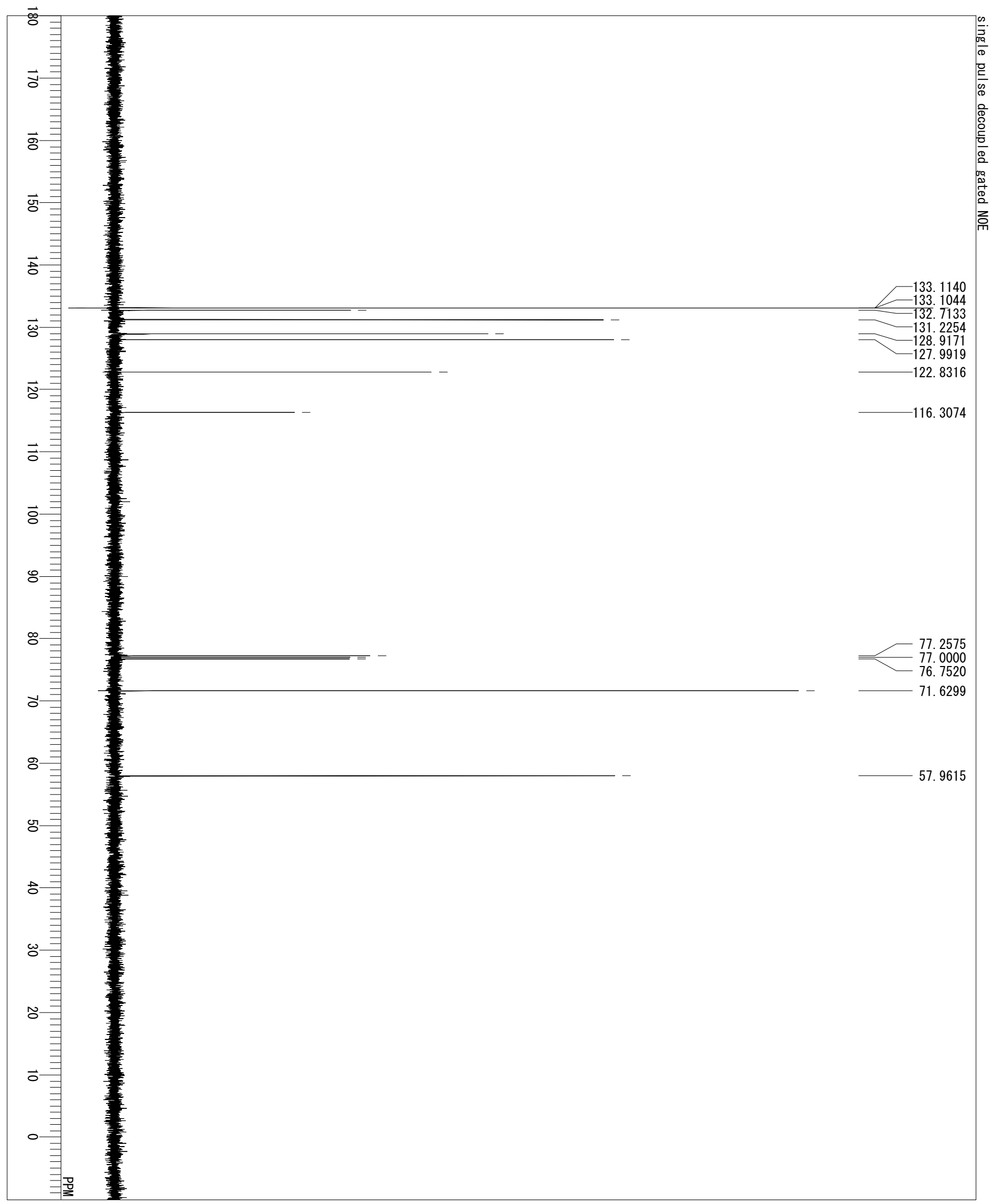

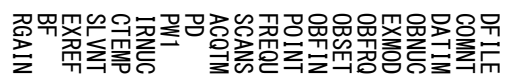

g $\vec{g} \quad$ s.

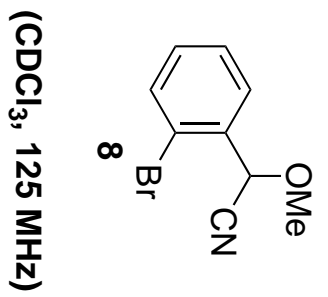

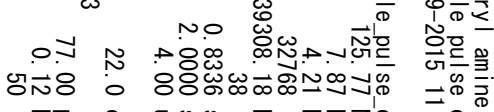

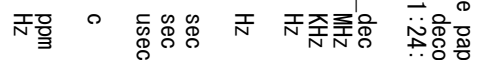

送要 


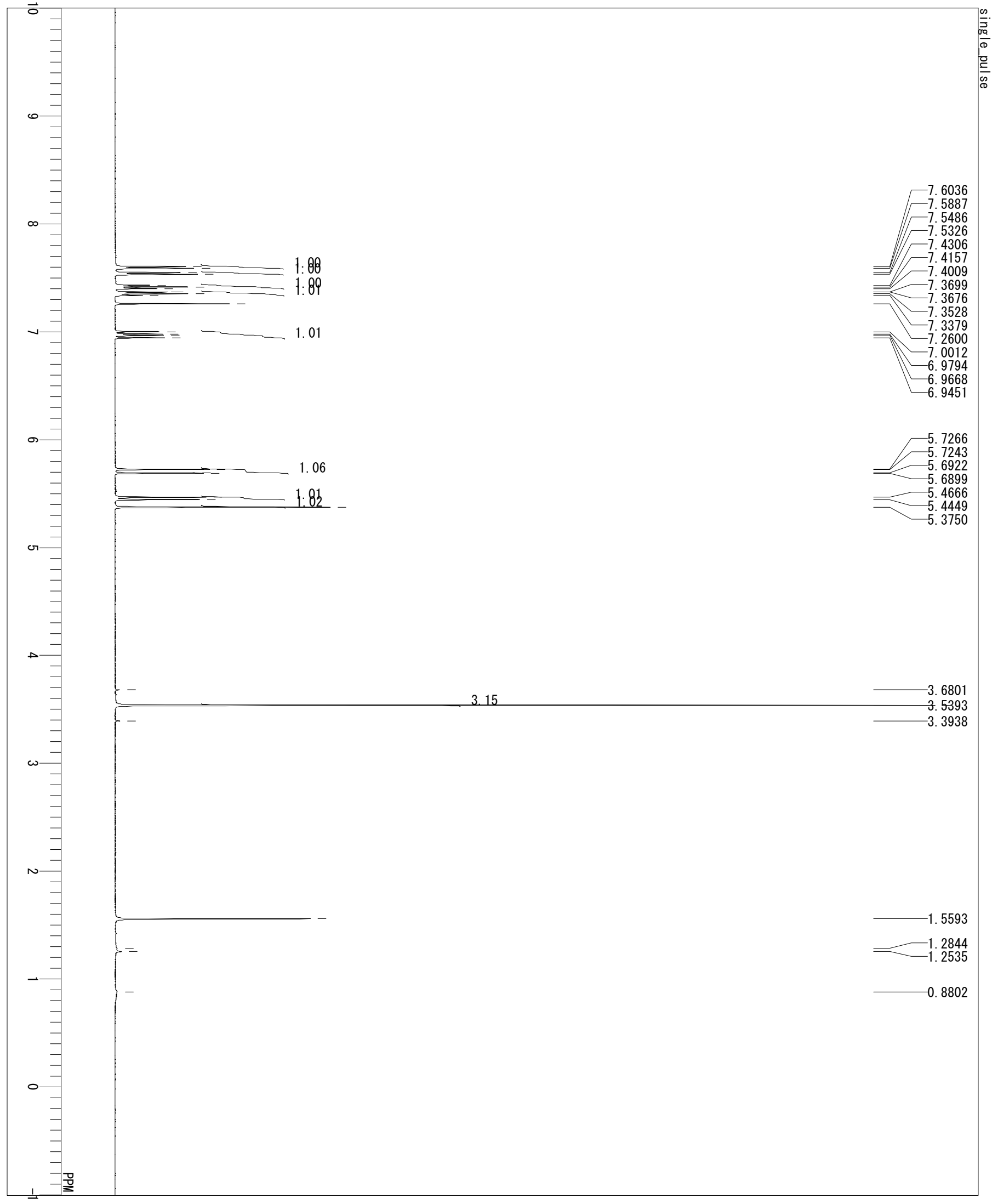

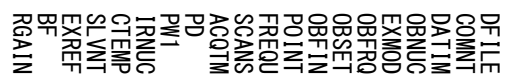
畄

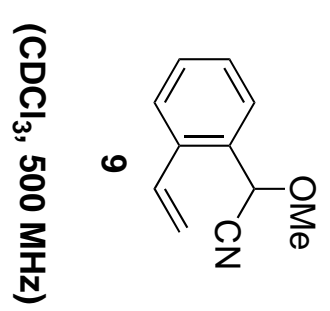




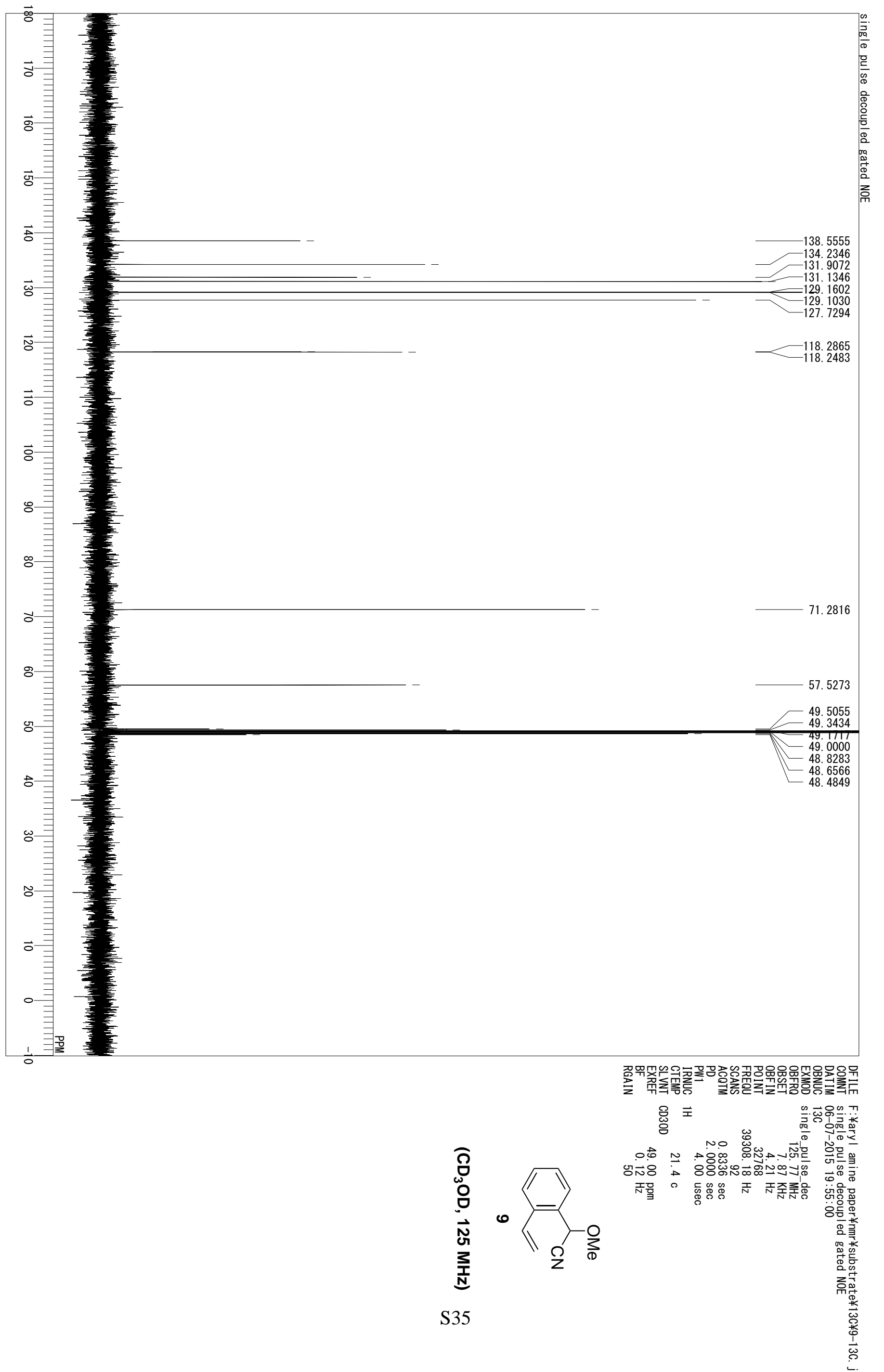




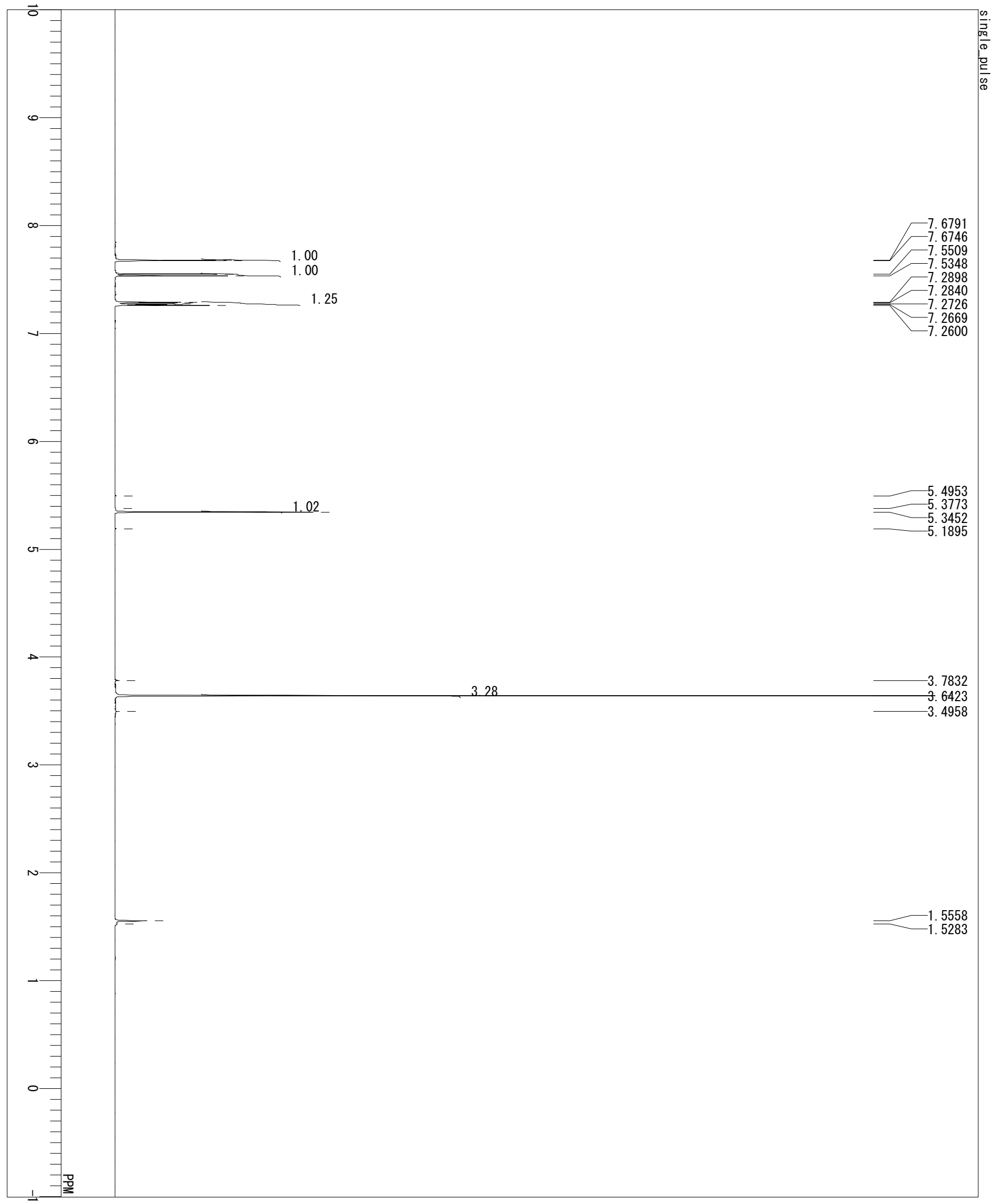

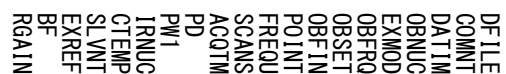
总

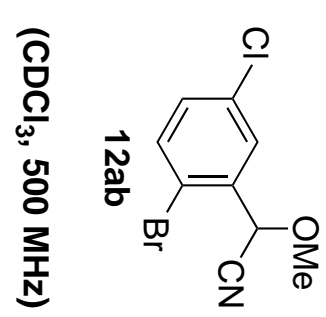

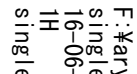

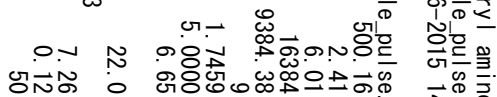

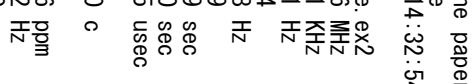
نं 


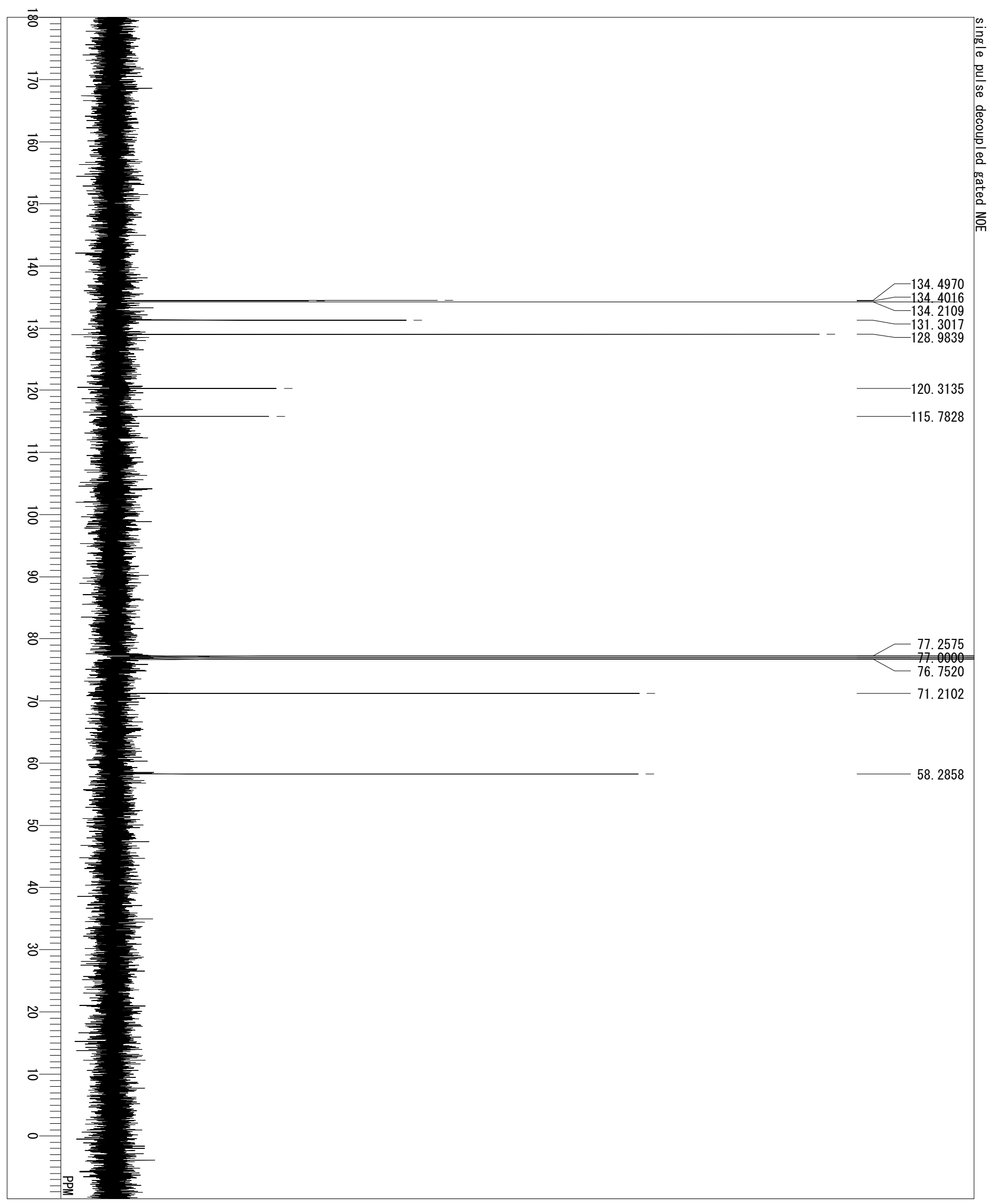

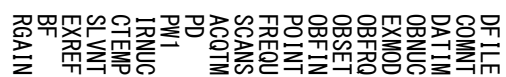

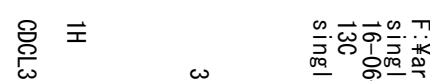<smiles>CC(=O)c1cc(O)ccc1C(=O)O</smiles>

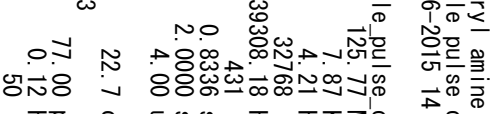

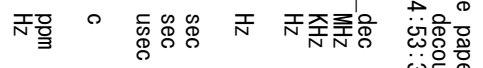




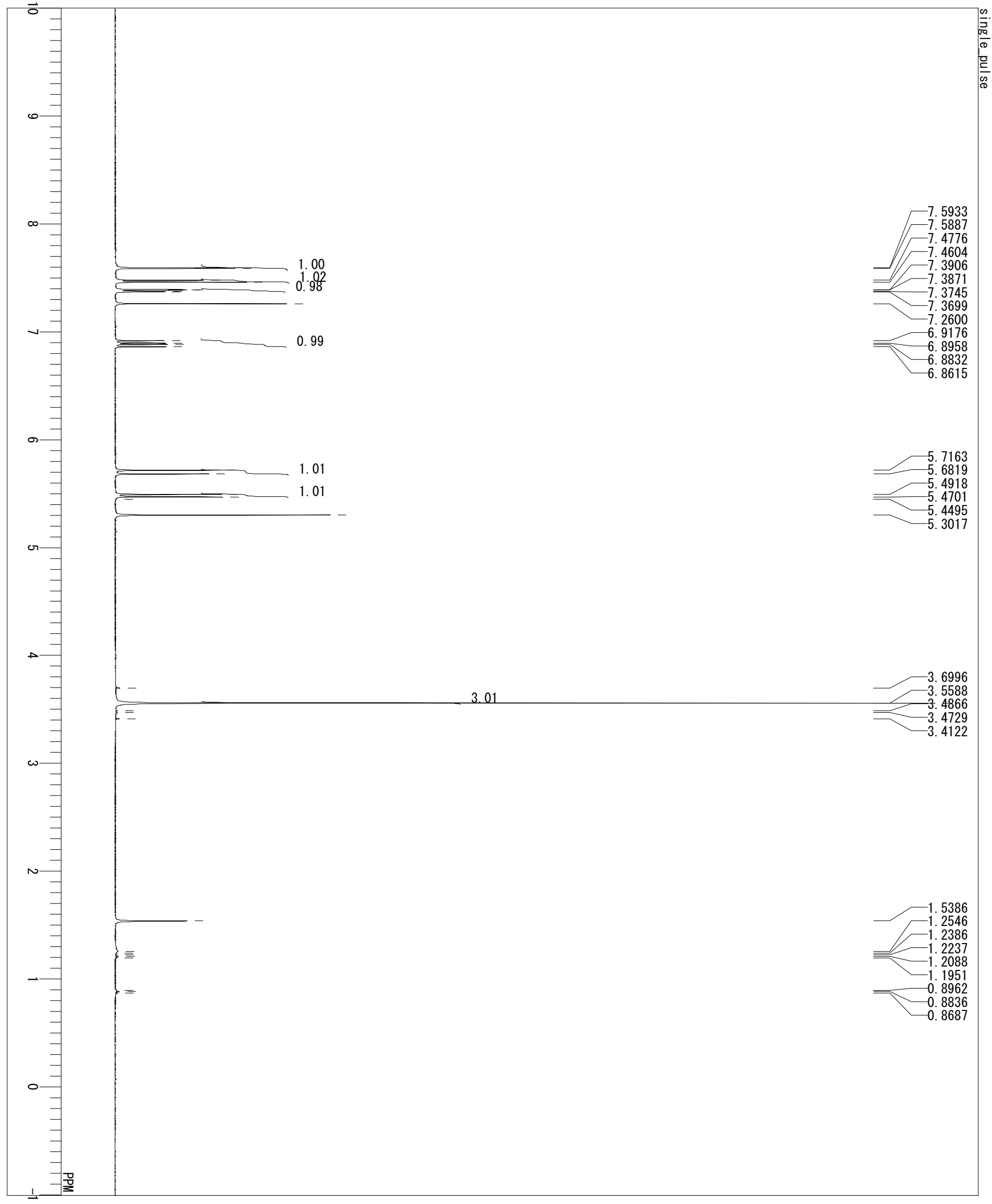

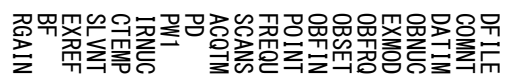

舀

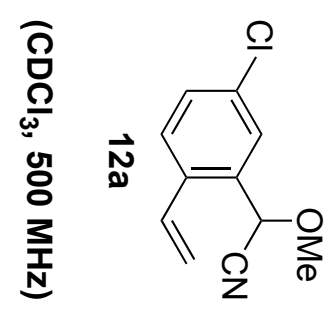

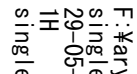

ㄱ. N

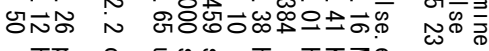

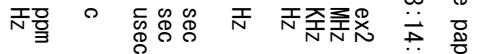




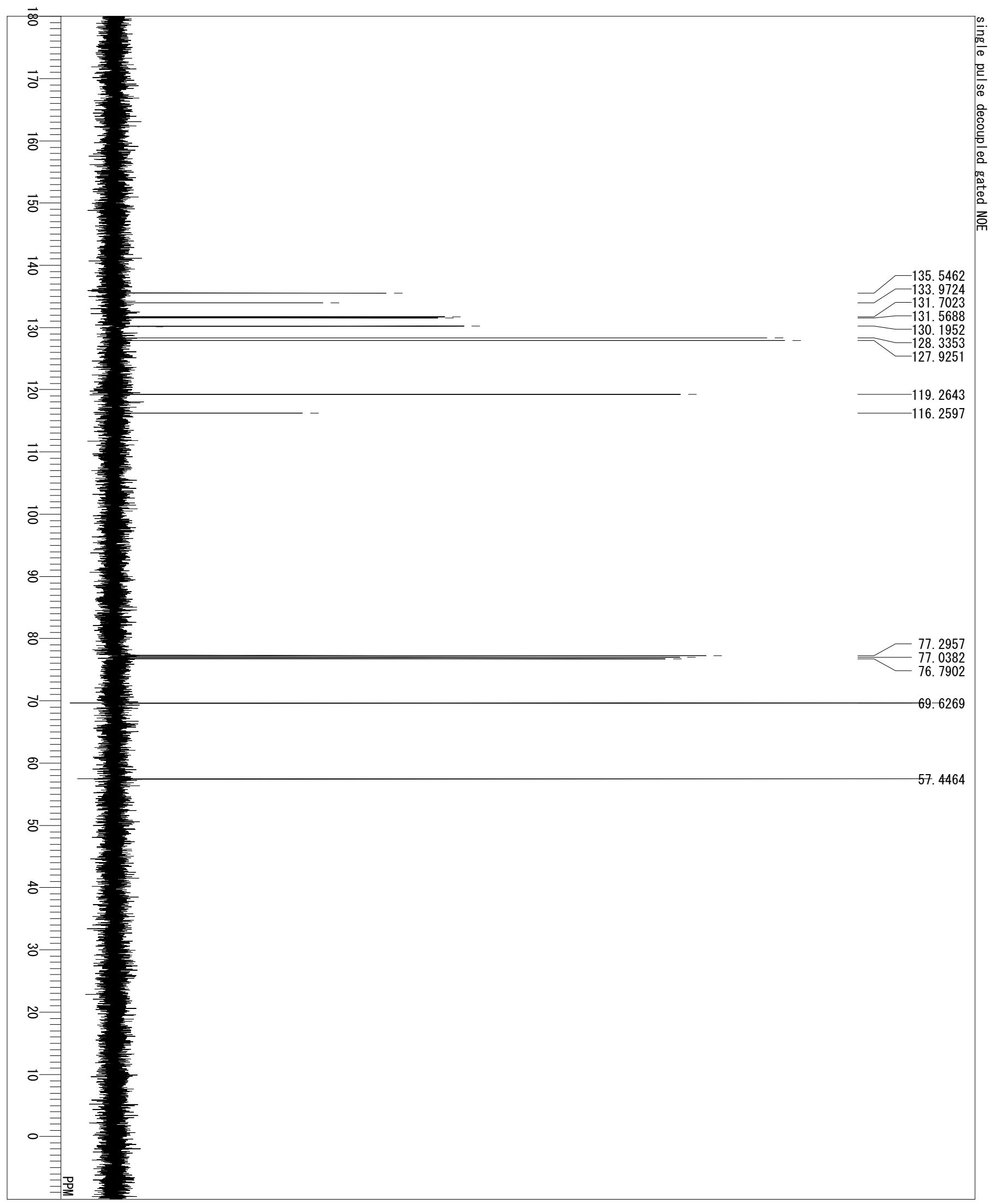

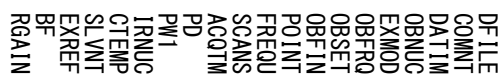

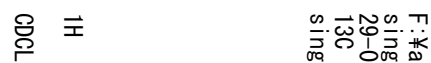

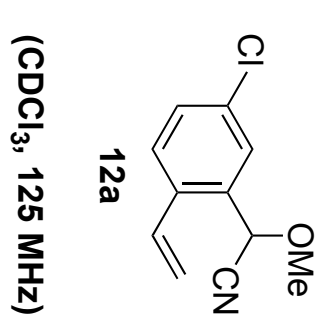

No $\mathscr{\omega}_{\omega}$ त

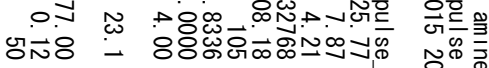

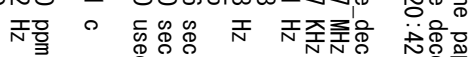

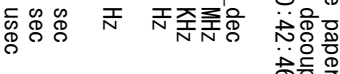




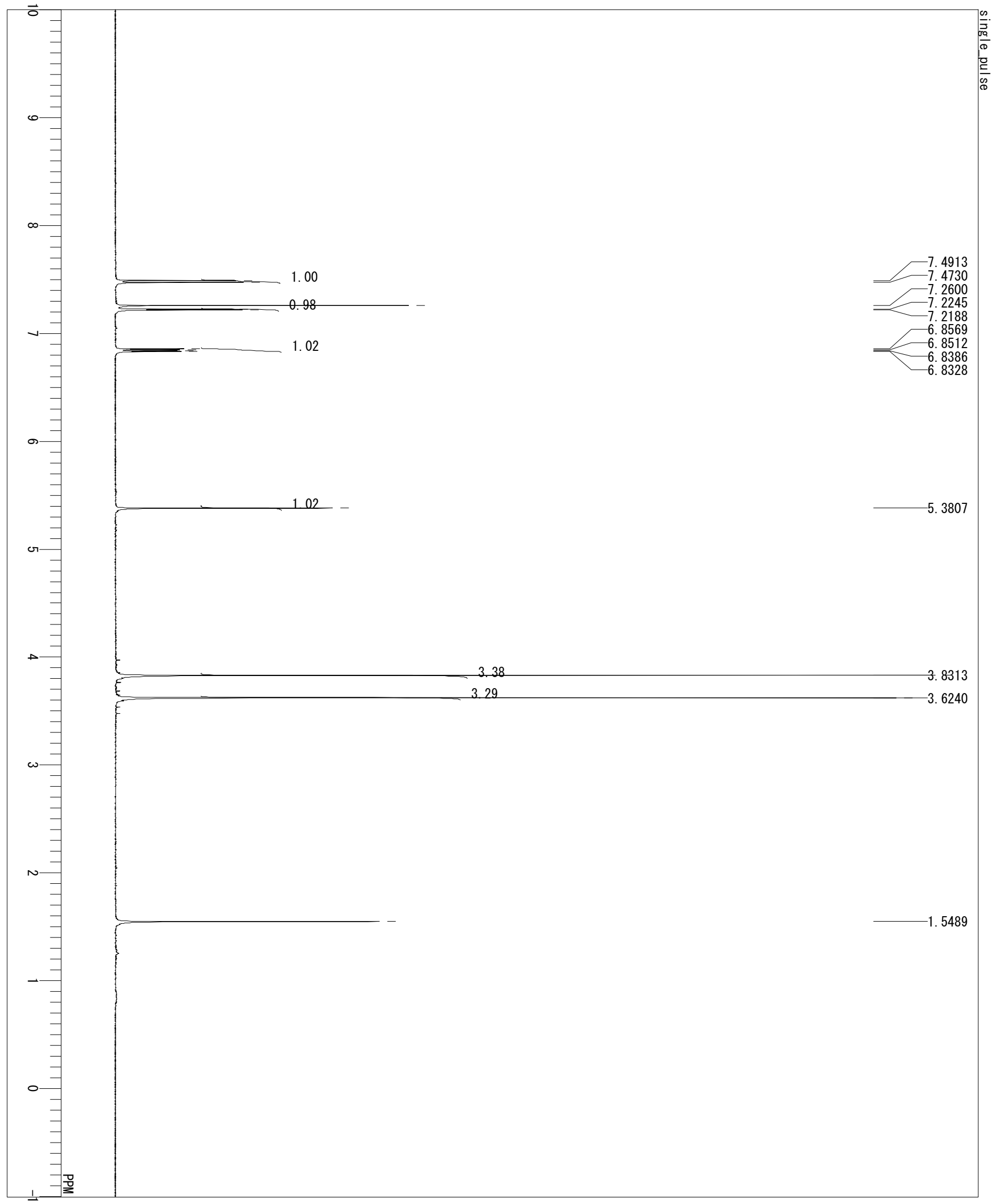

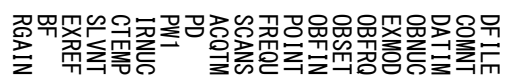

善

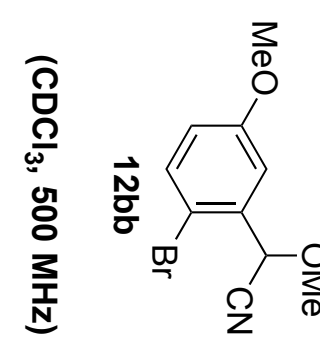

a)

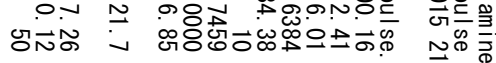

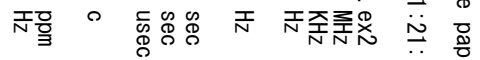

苻 


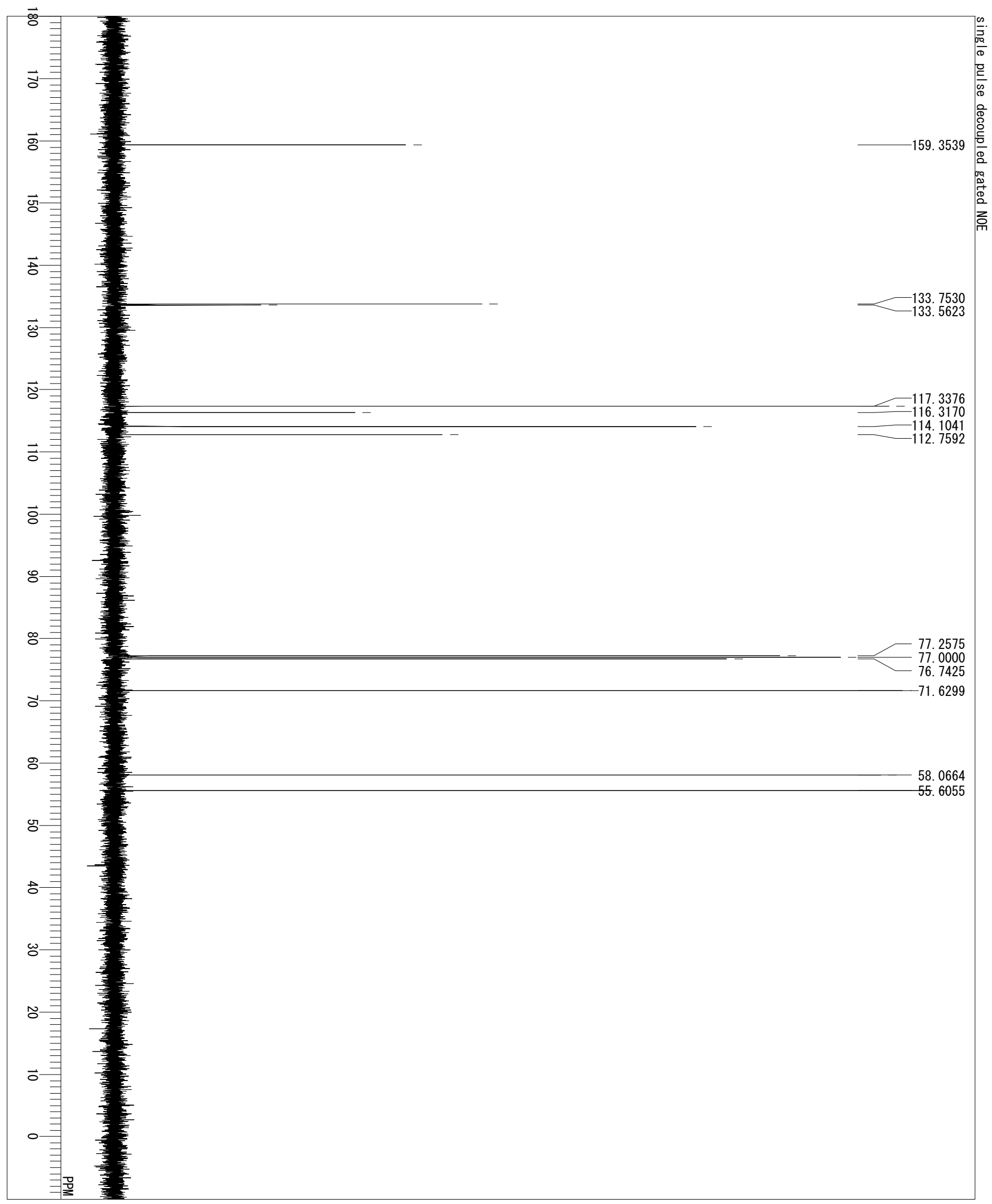

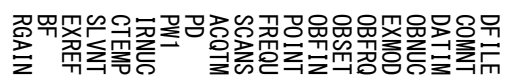

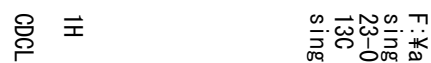

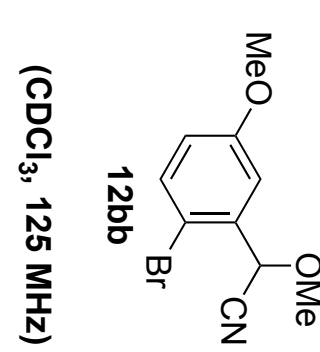

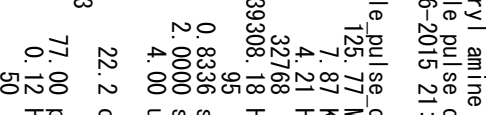

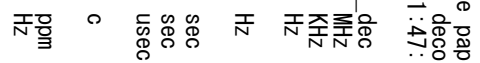




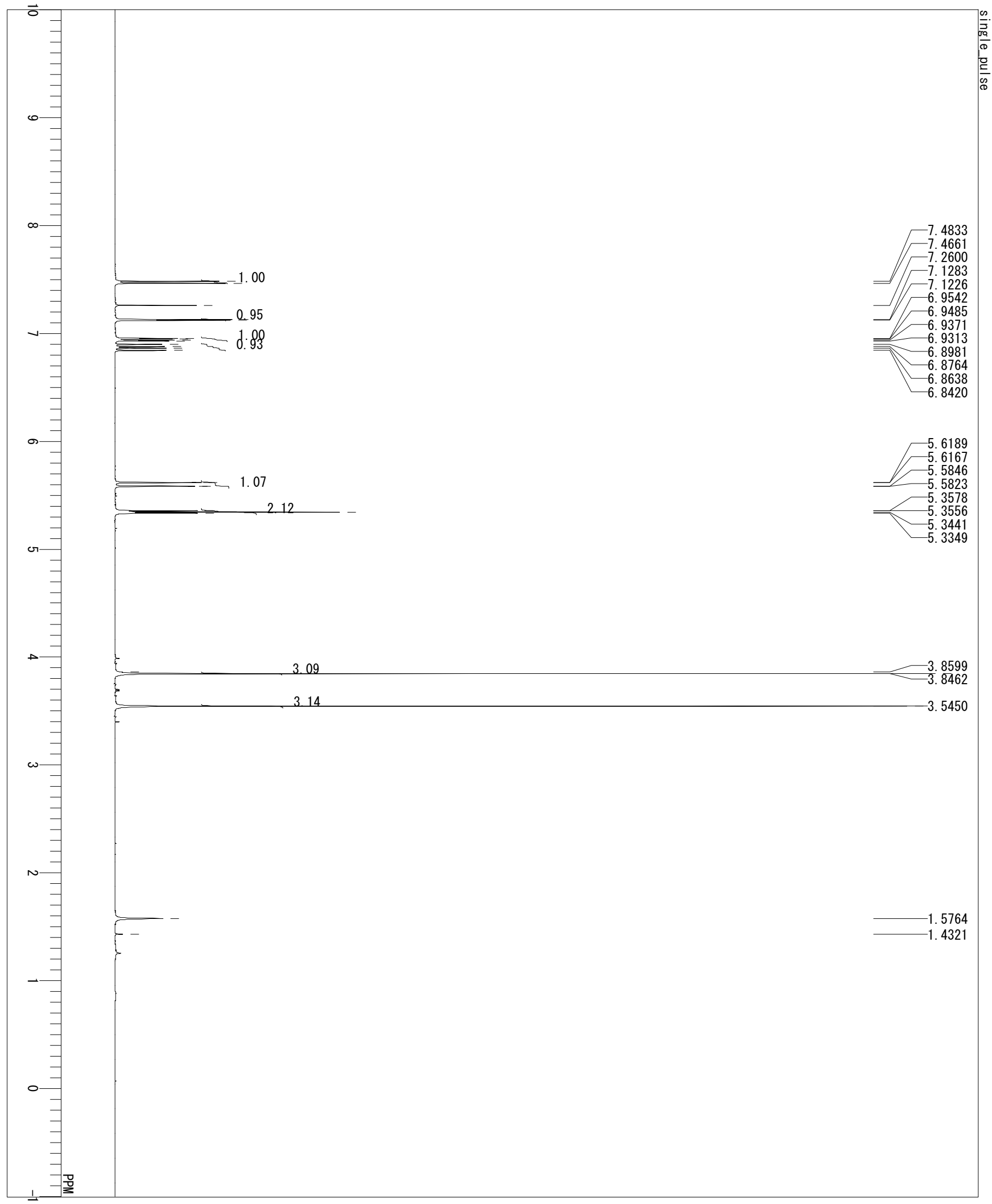

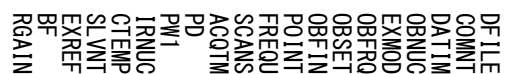
总

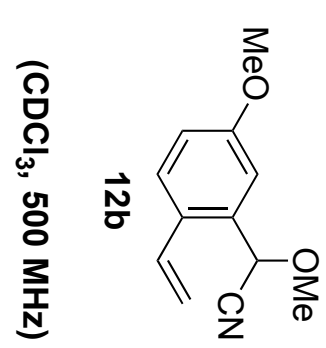

ज. 귱쇼 म

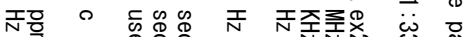




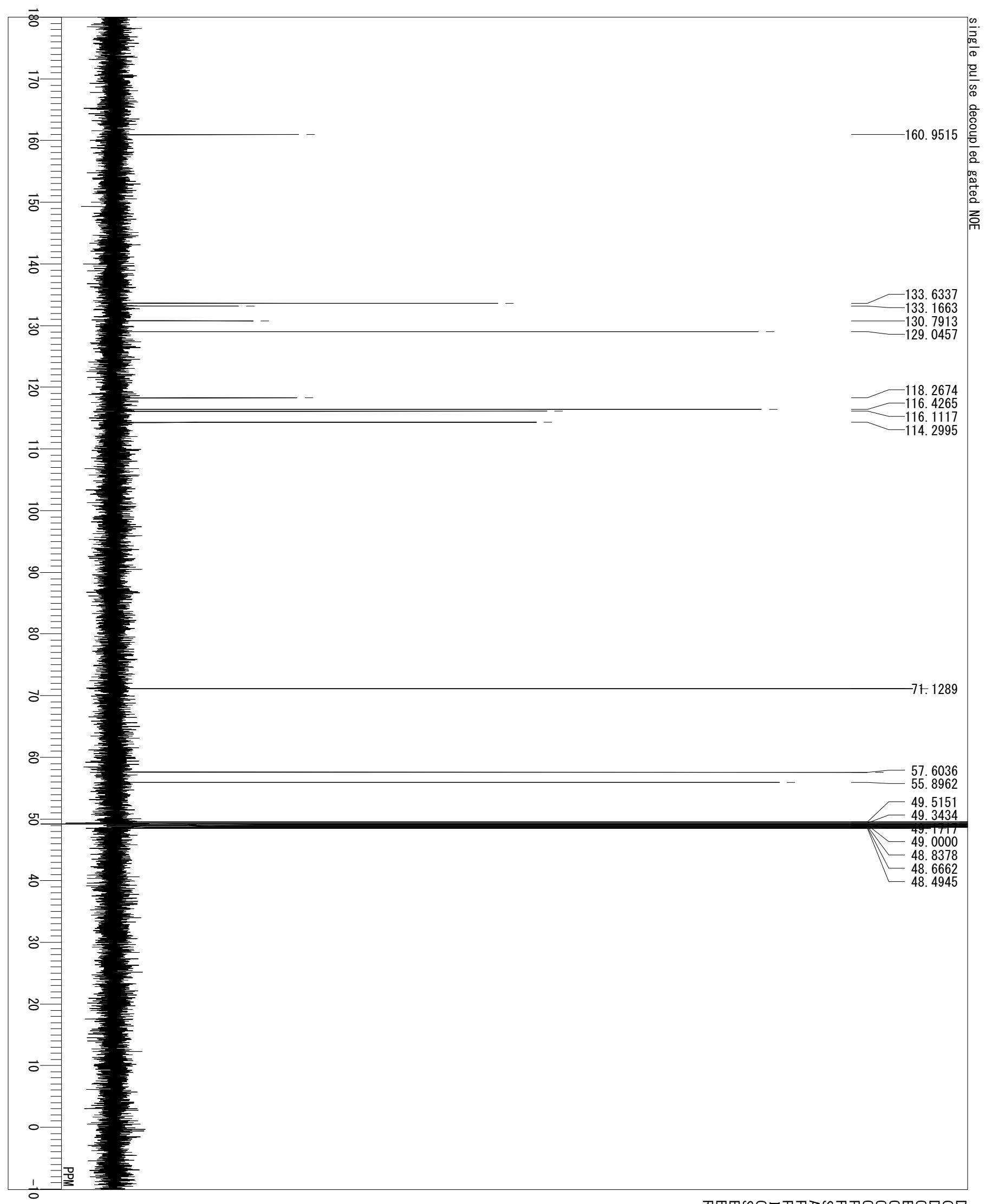

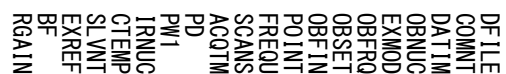

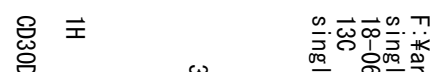<smiles>C=Cc1ccc(OC)cc1C(O)OC</smiles>

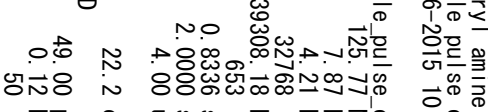

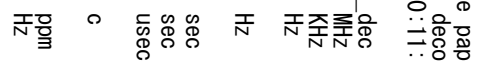




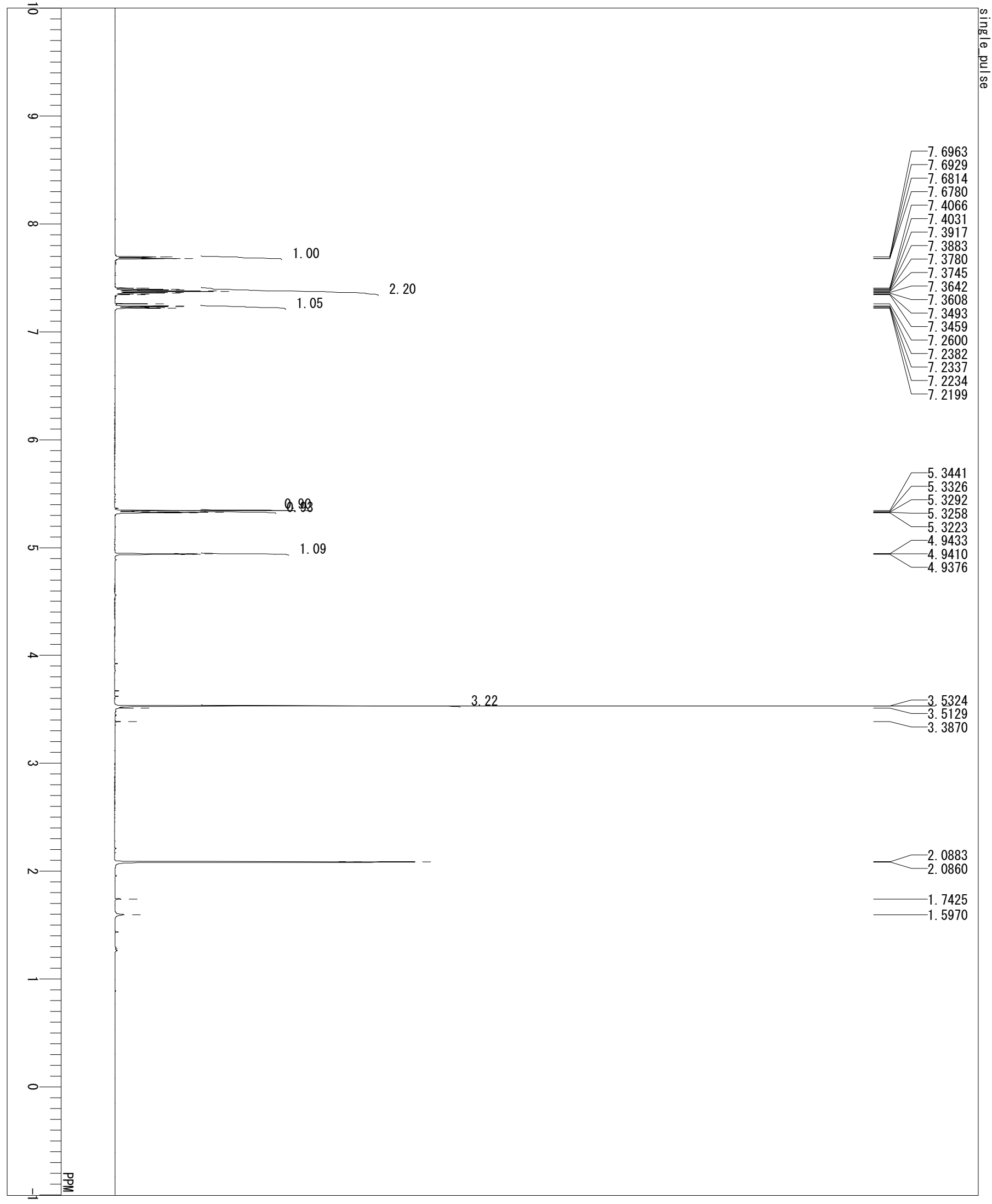

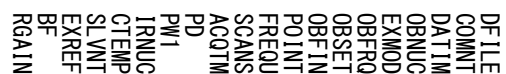

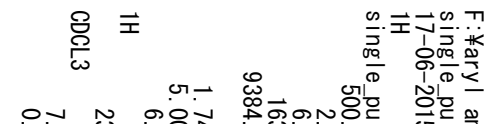

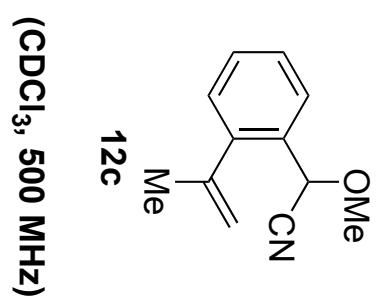

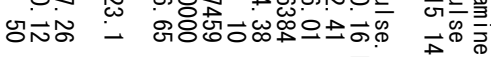

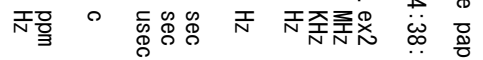




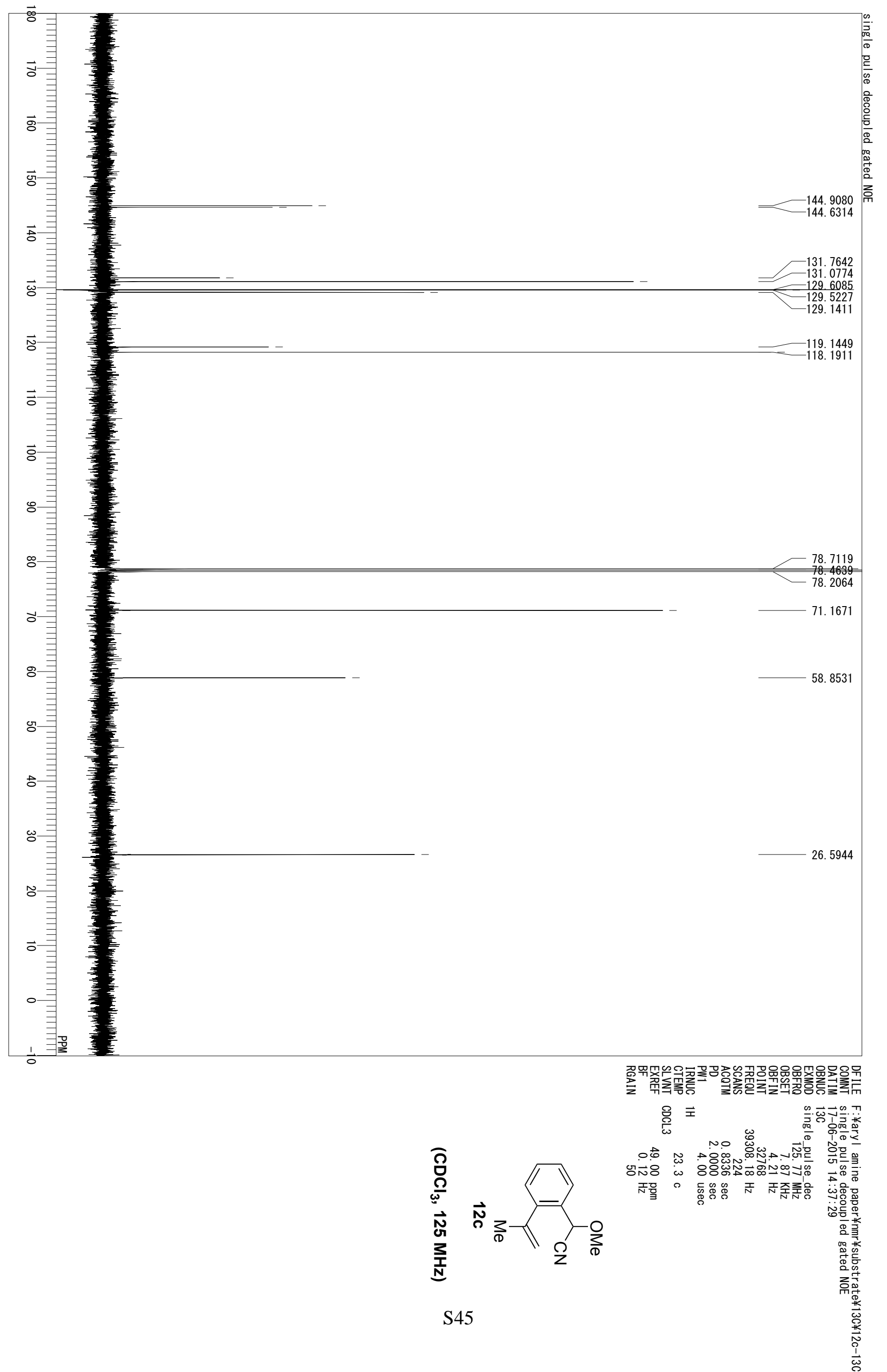




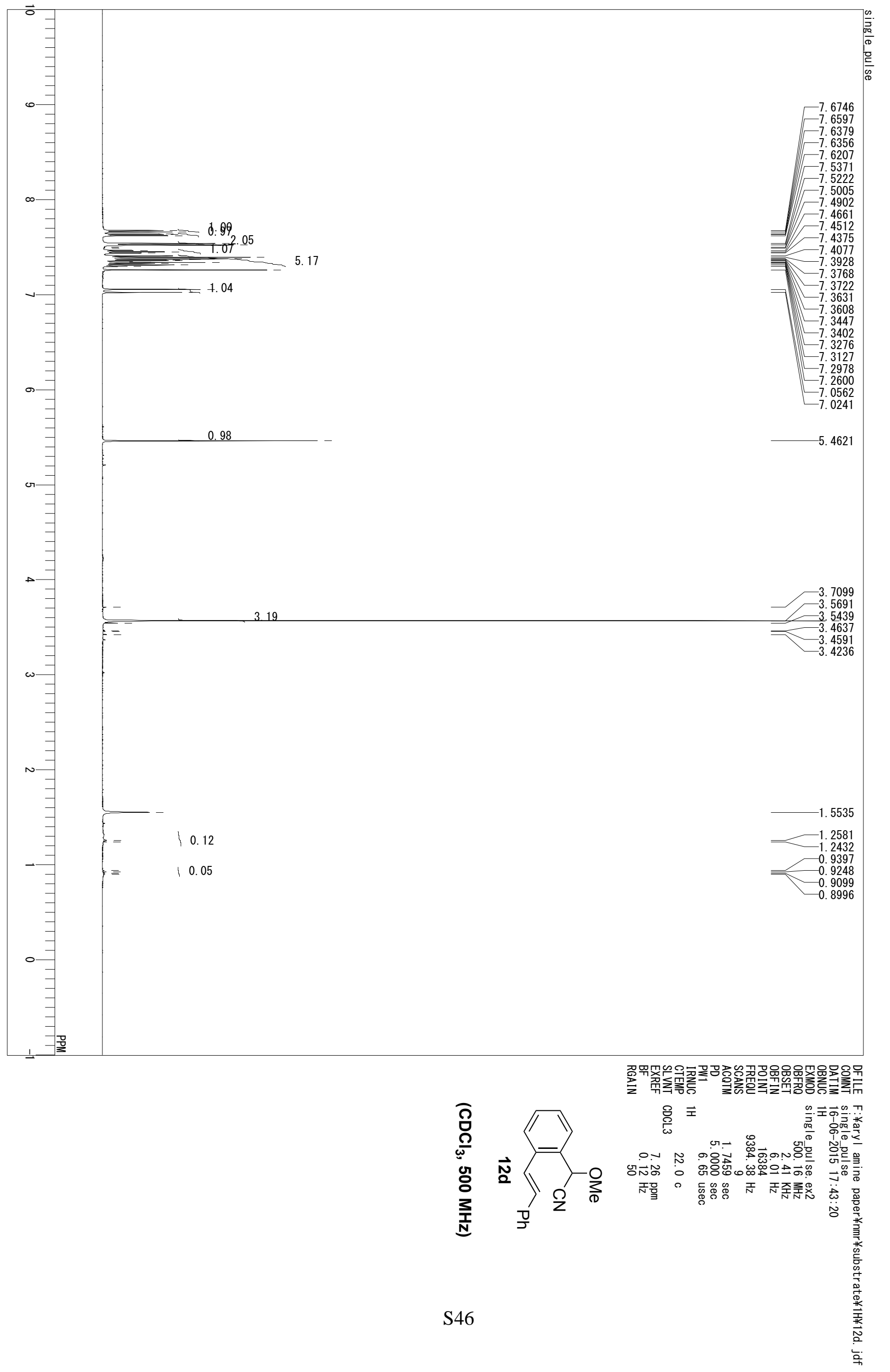




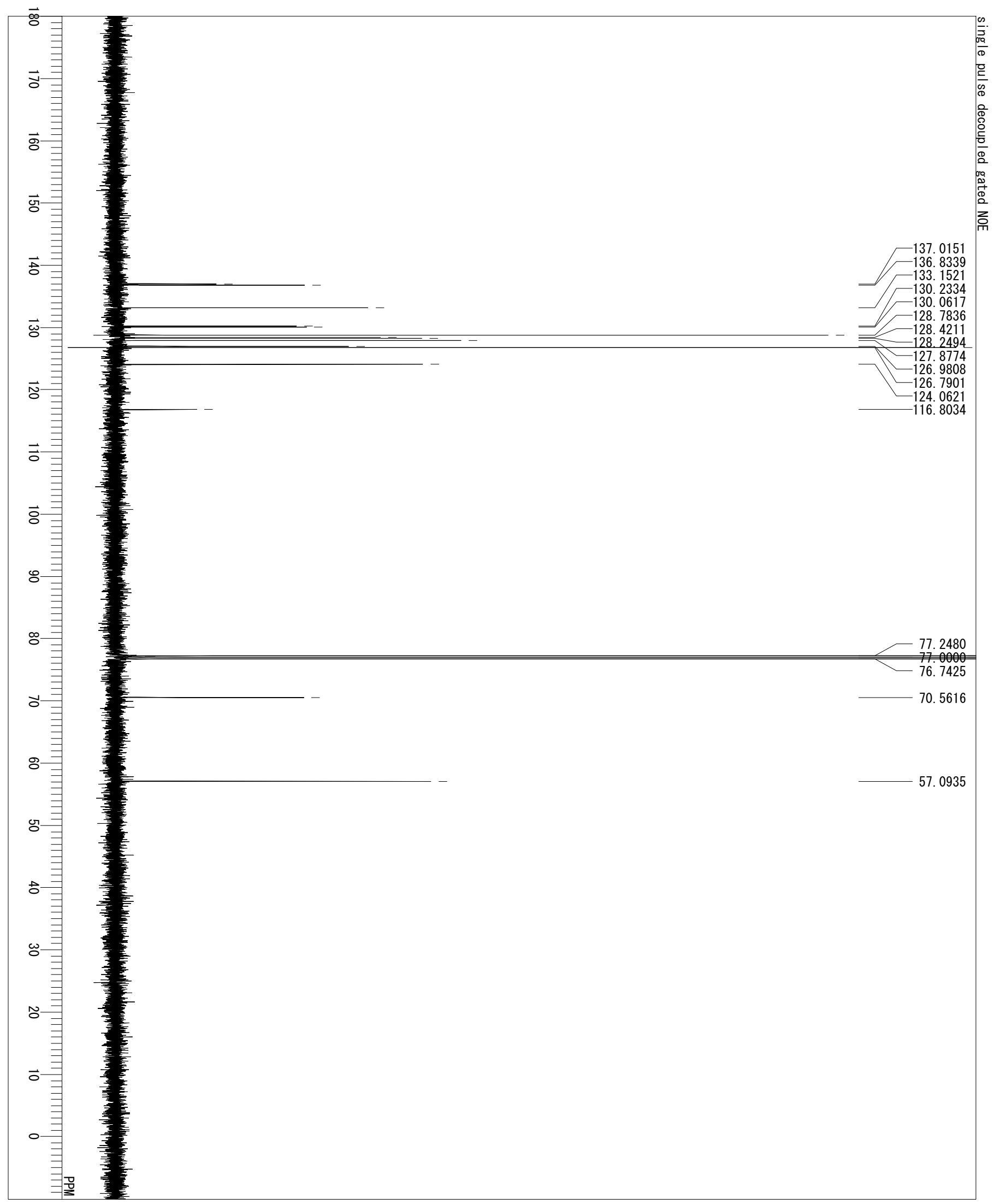

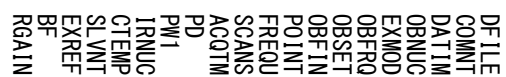

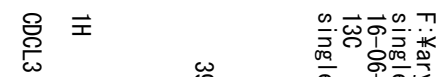

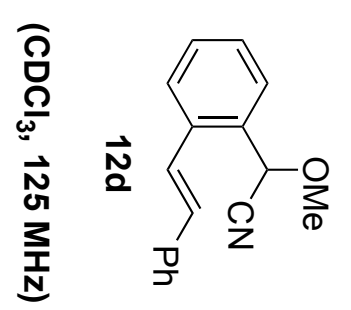

o. जñ

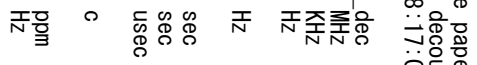

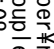




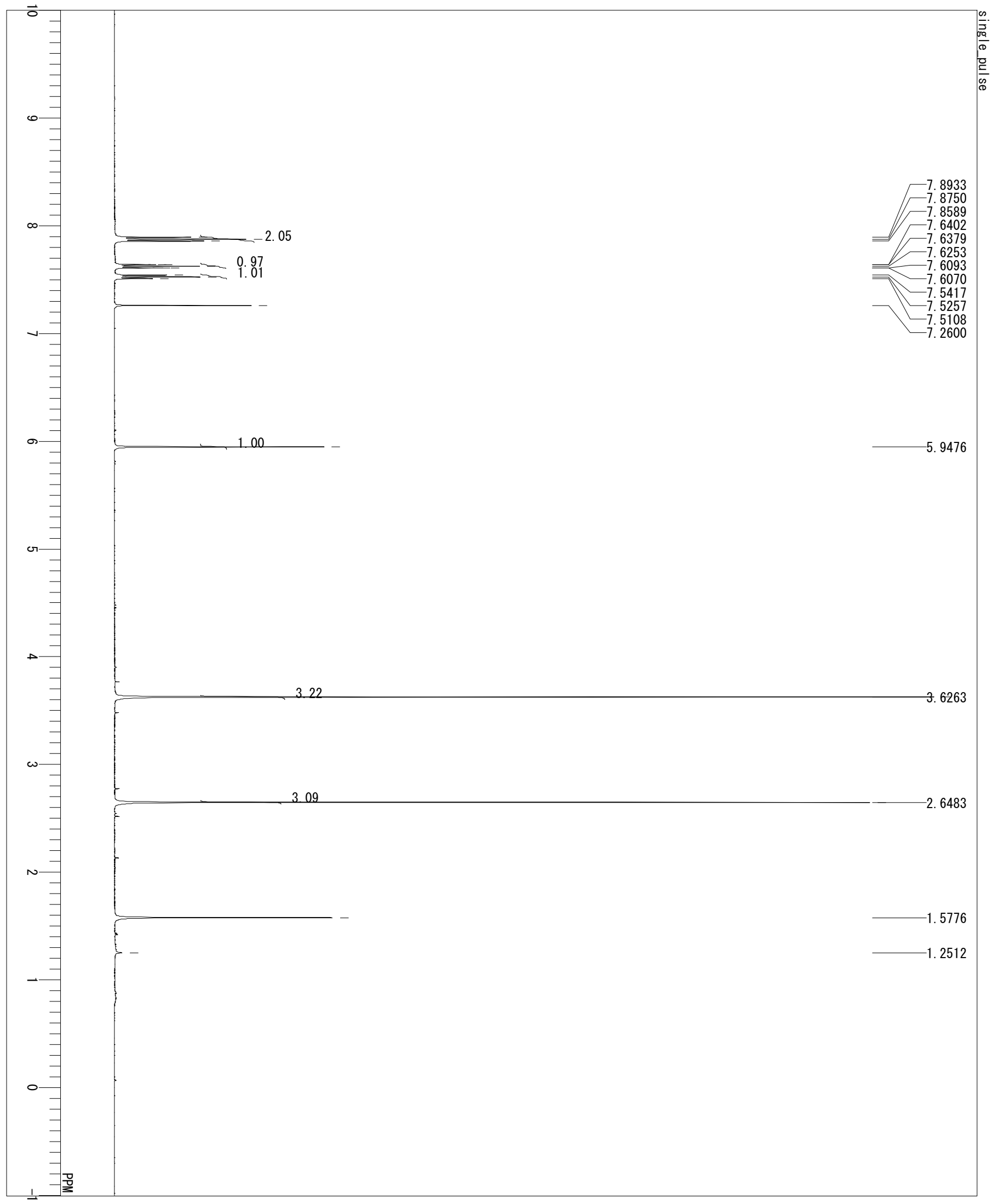

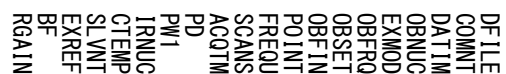

$\underset{\varrho}{\stackrel{\varrho}{\omega}} \vec{x}$

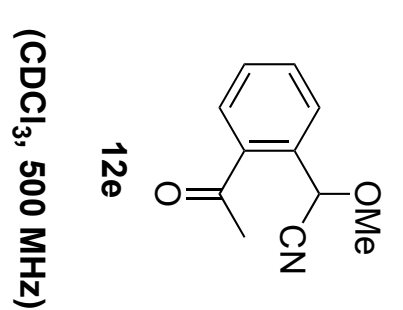

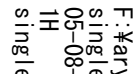

ㅇ. 당

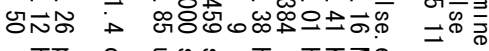

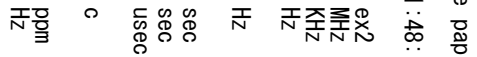




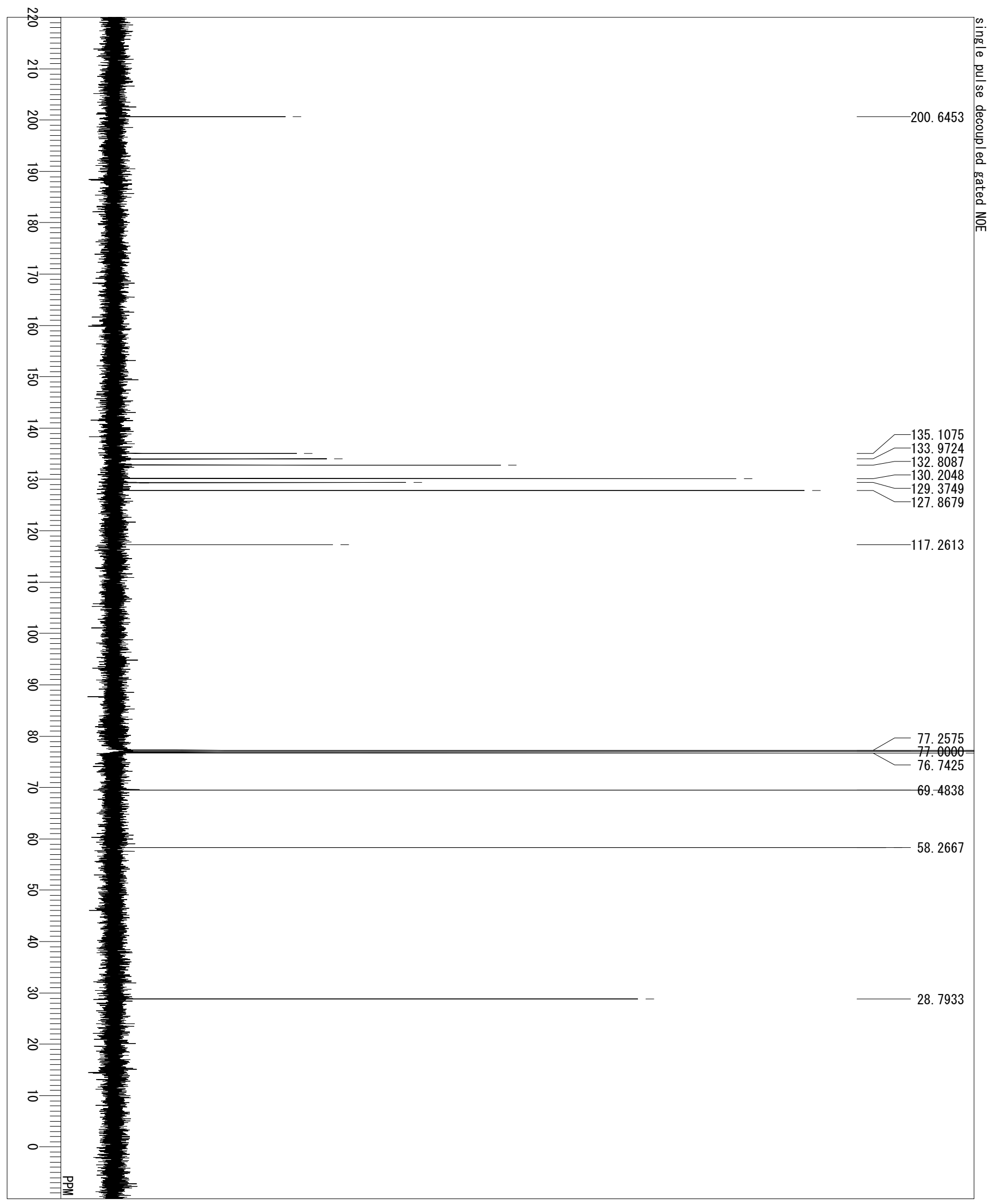

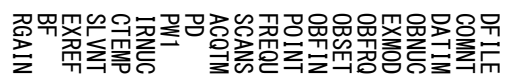

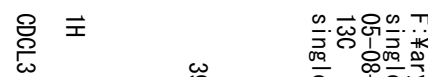

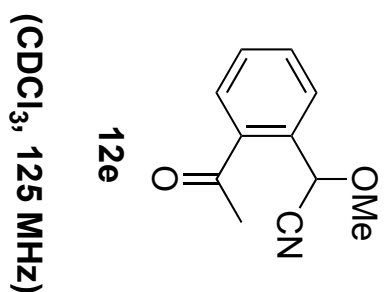

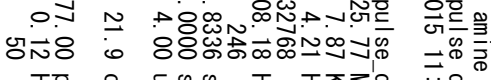

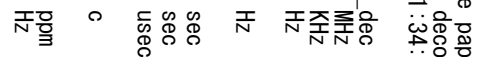




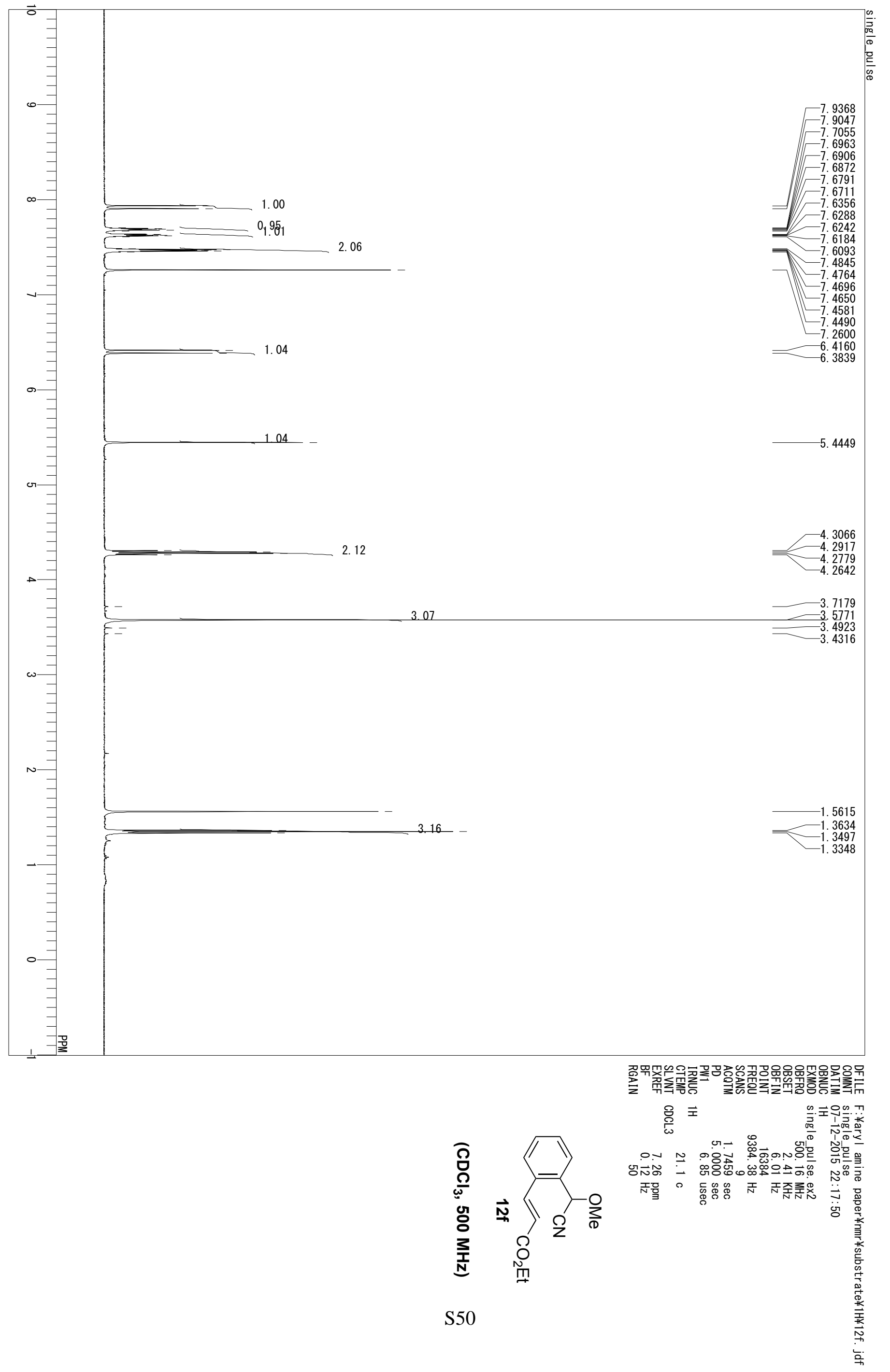




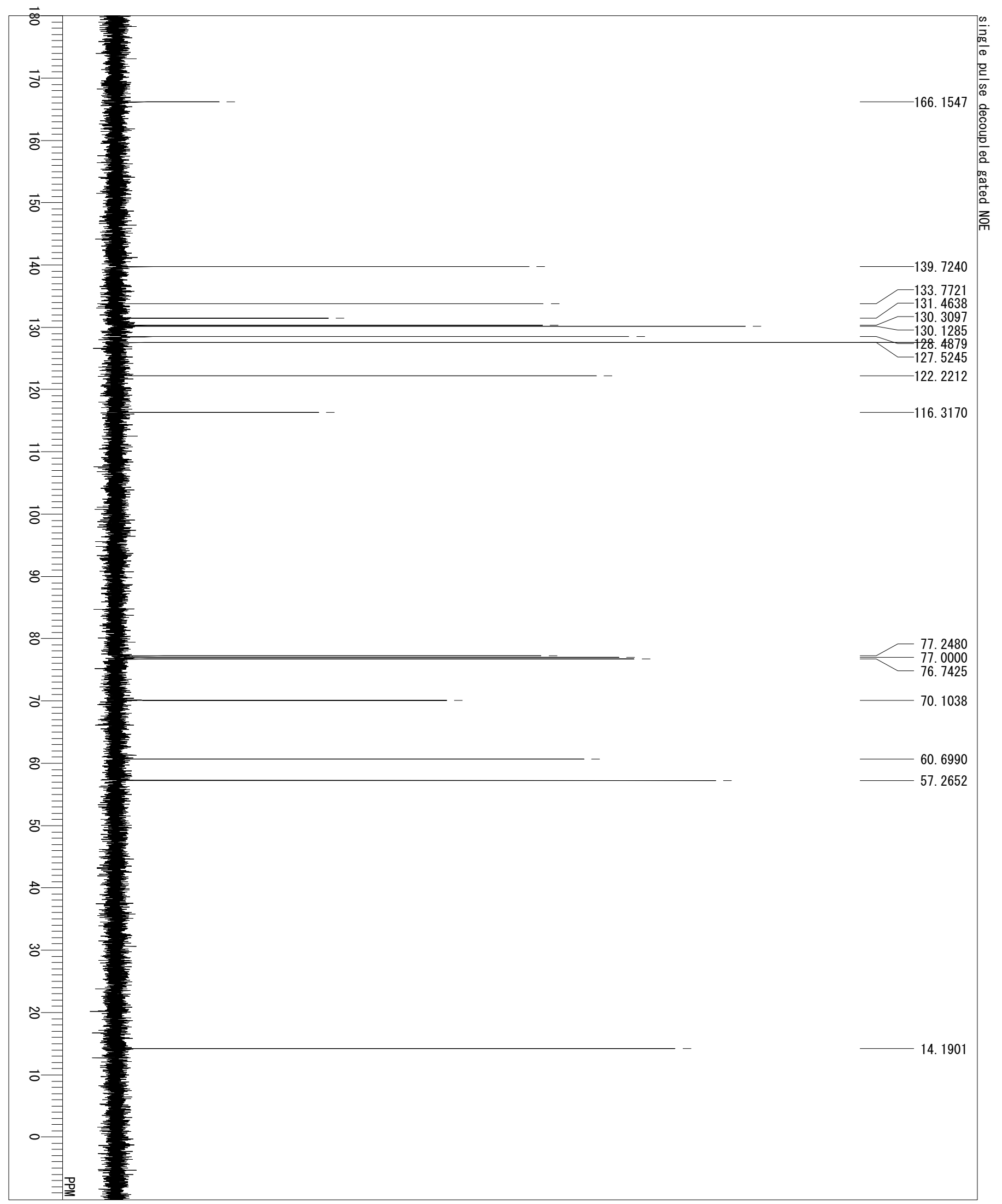

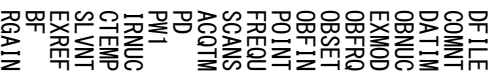

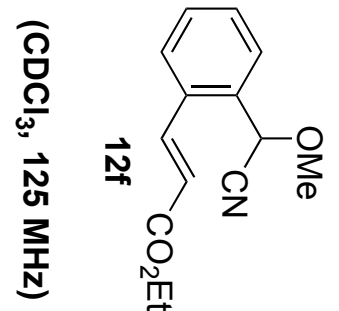

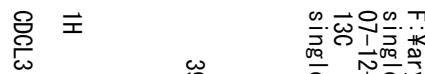

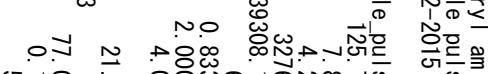

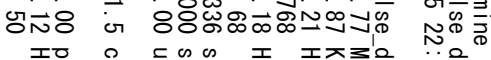

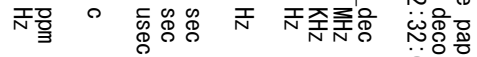

응 은 


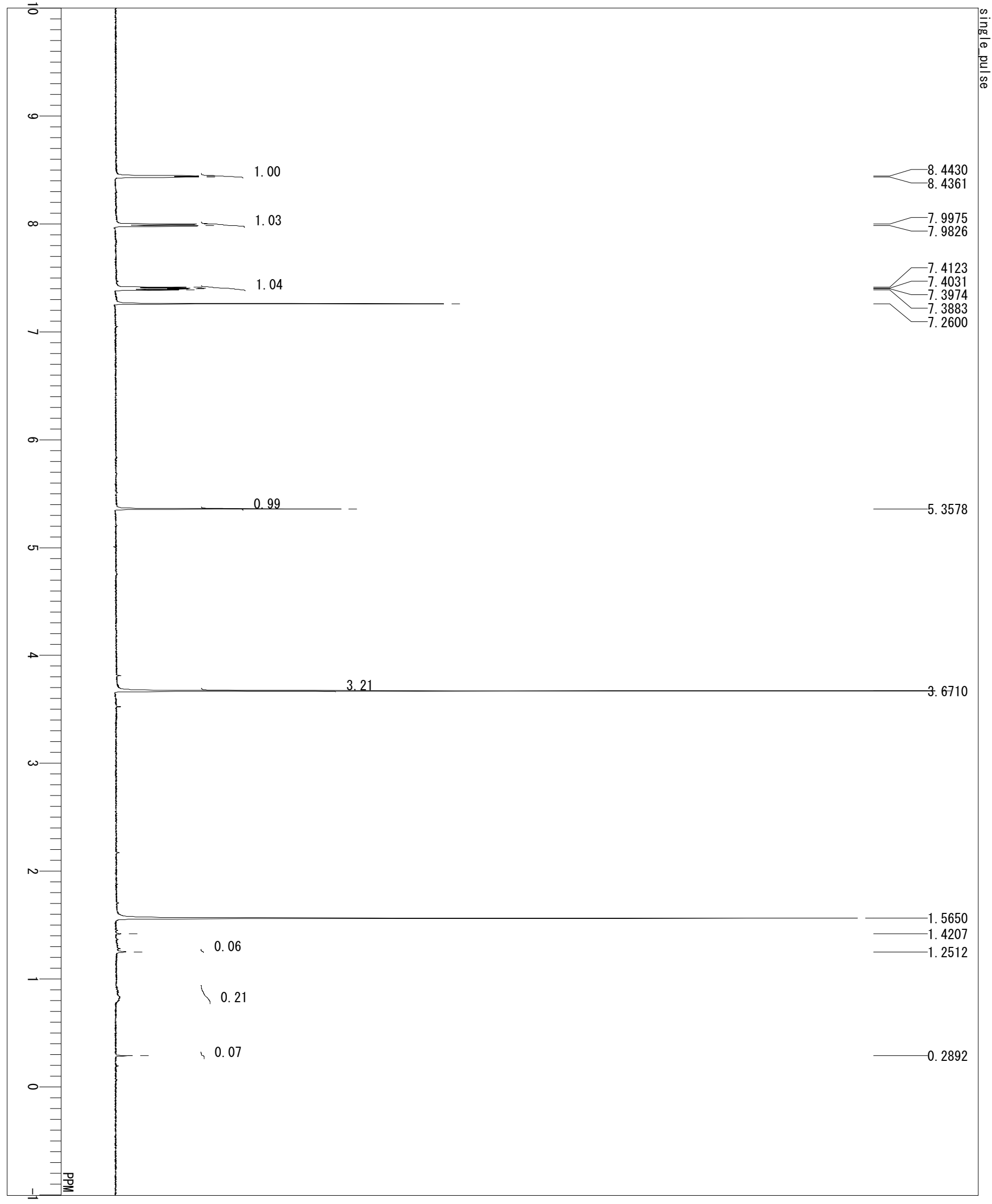

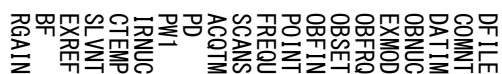

$\frac{8}{\omega} \vec{c}$

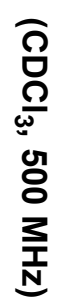

옥도오에 $\pi$

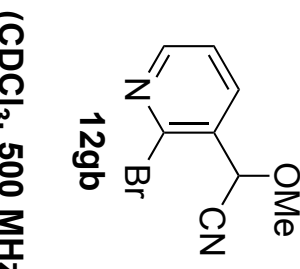

on 0 ar

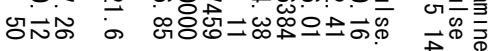

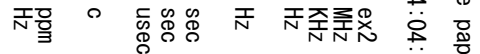




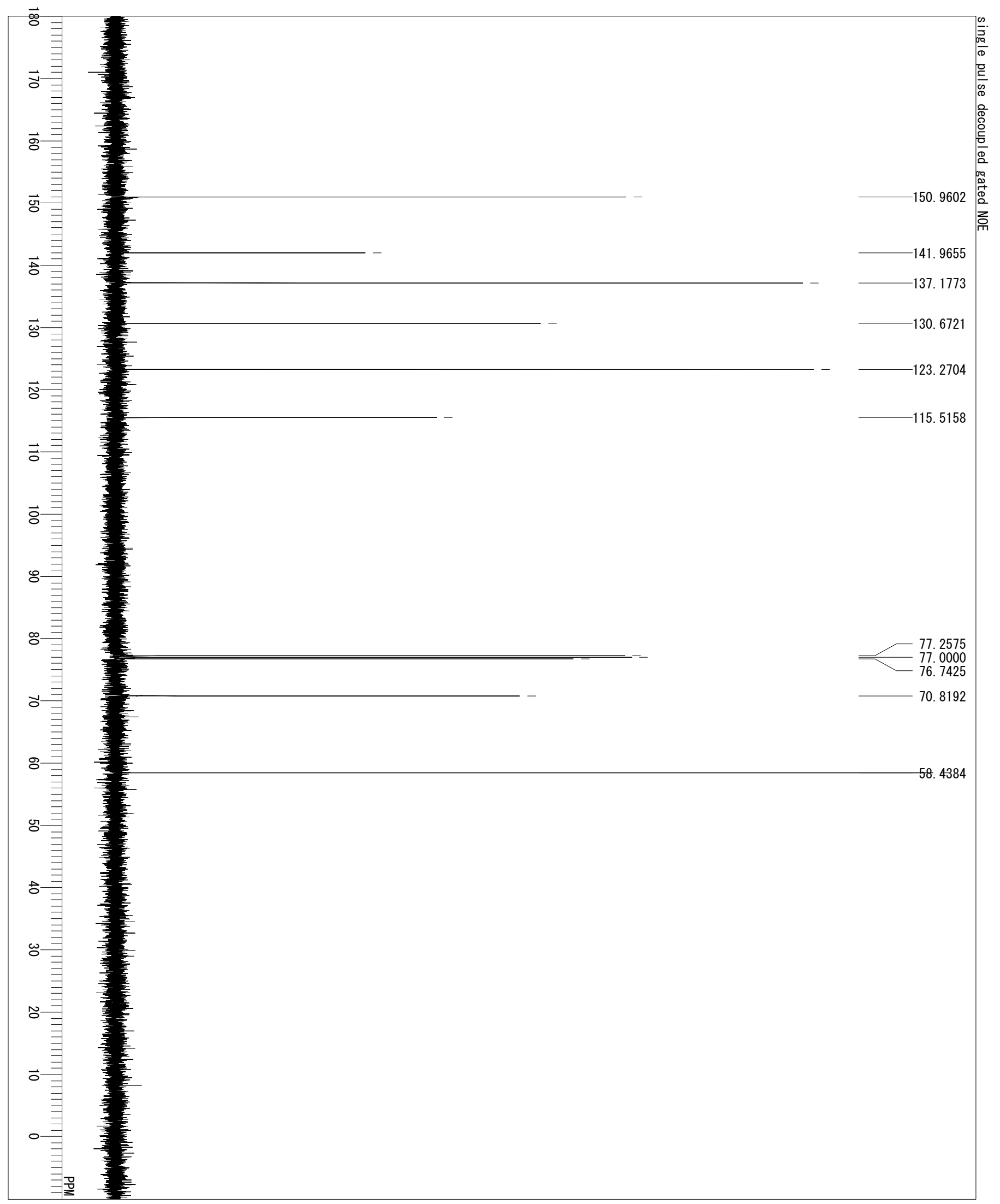

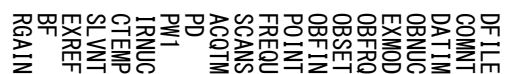
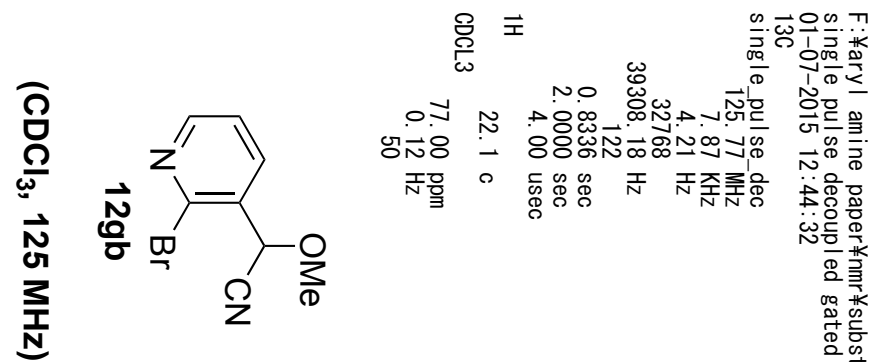

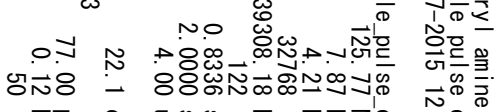

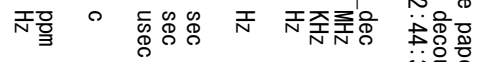

넌응 훈 


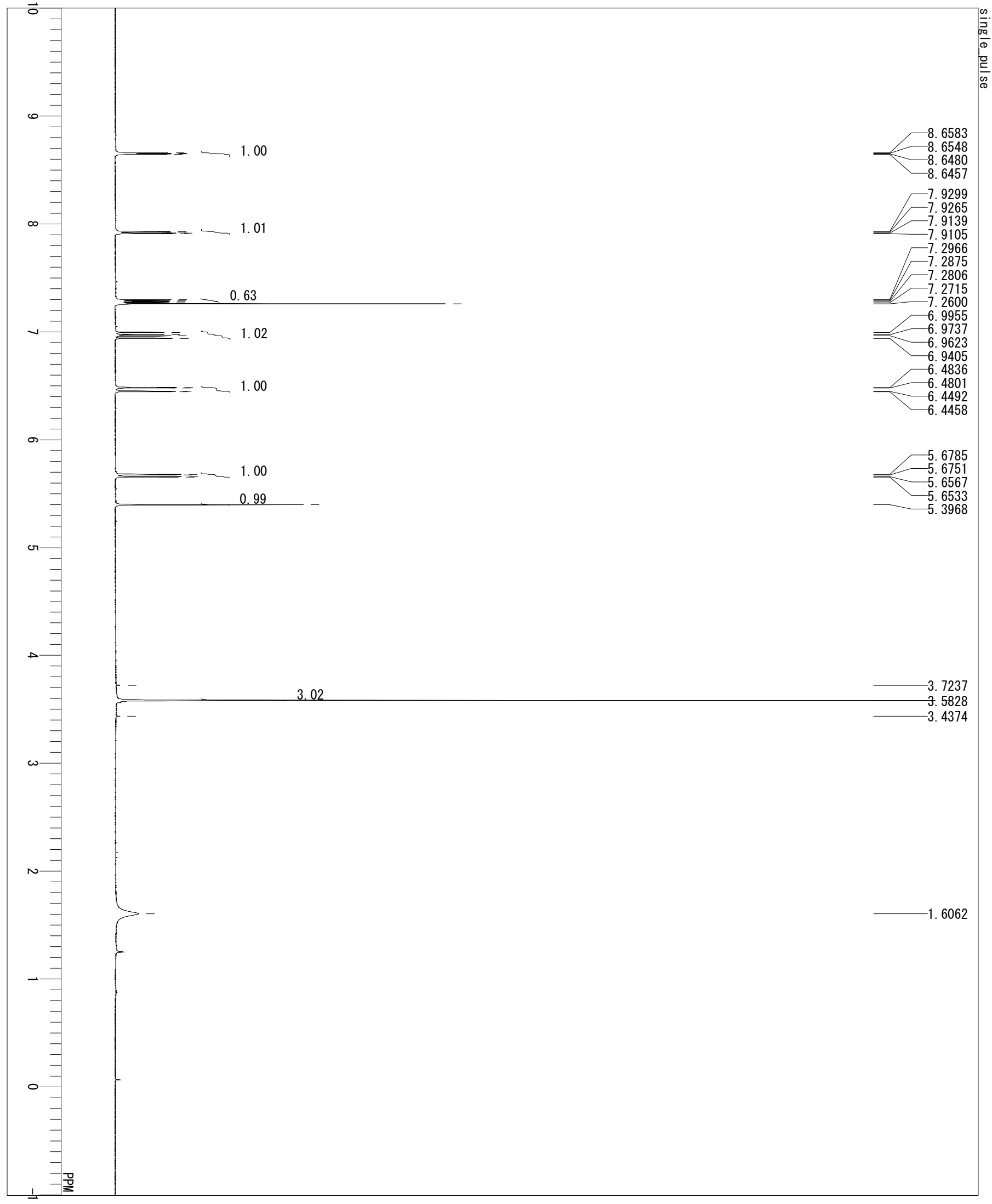

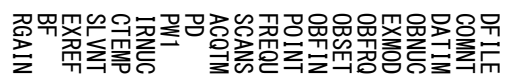

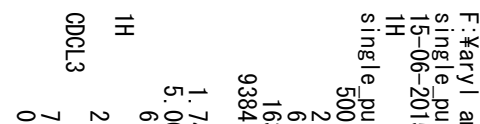

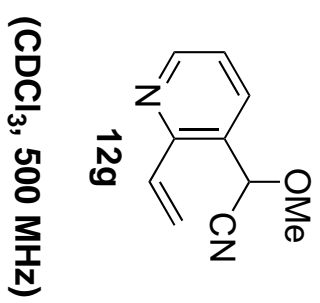

S54

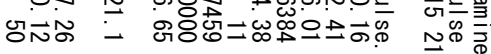

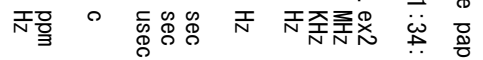




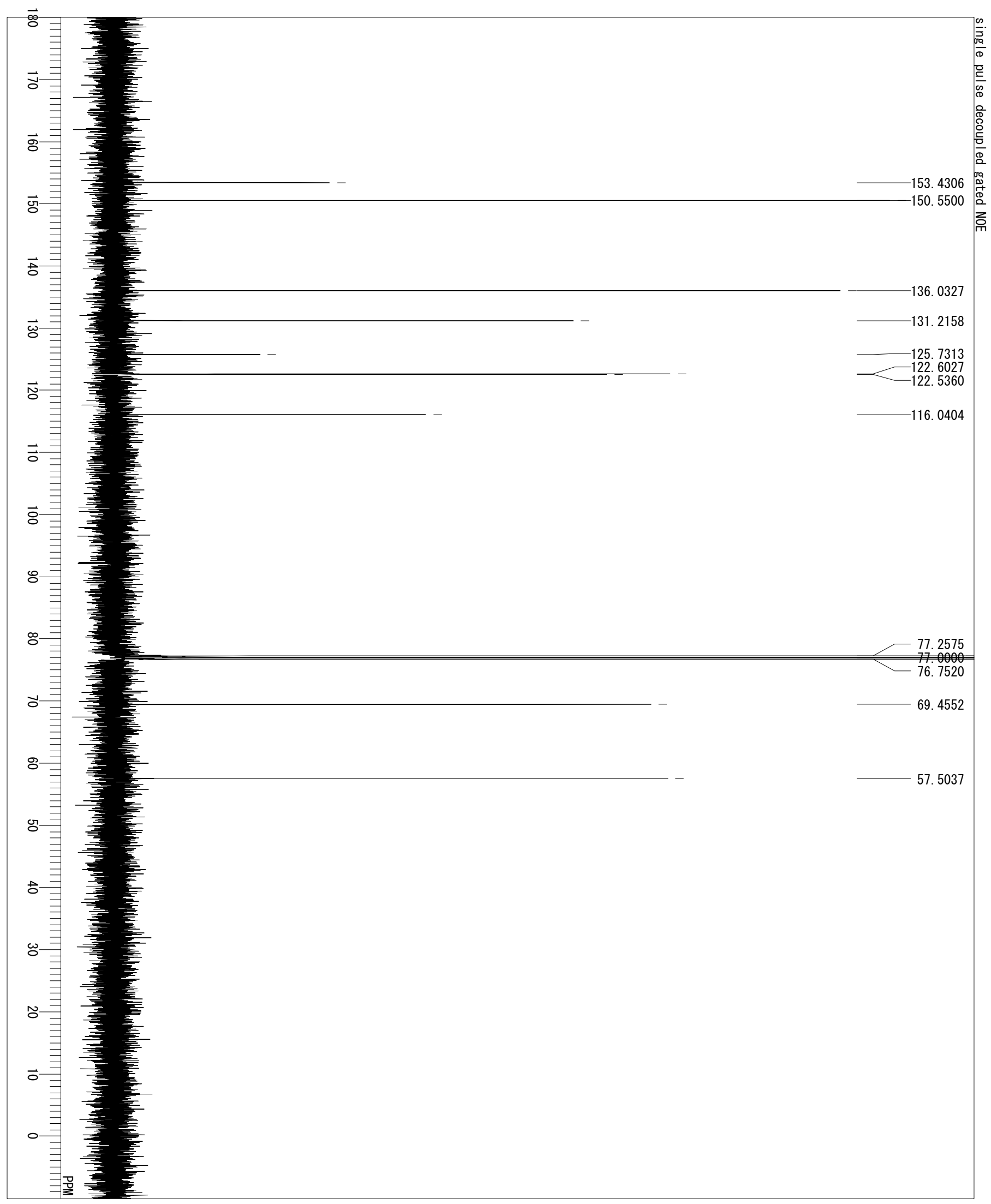

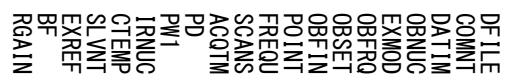
总现)

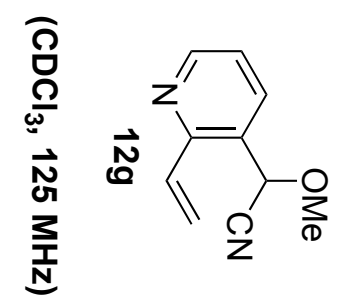

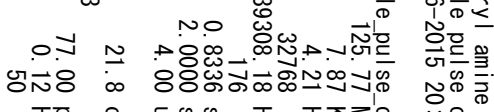

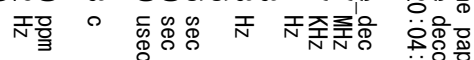




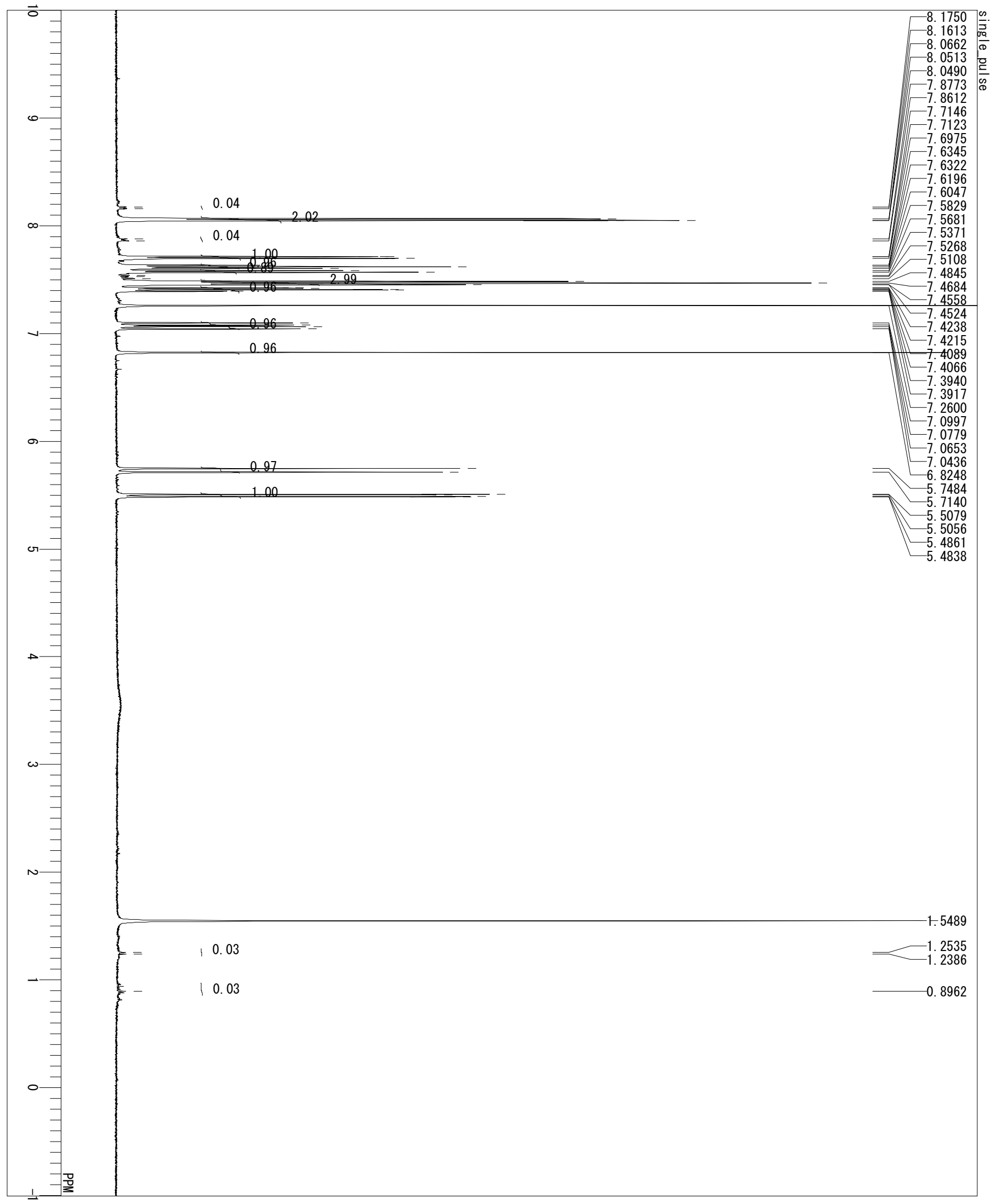

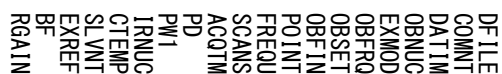

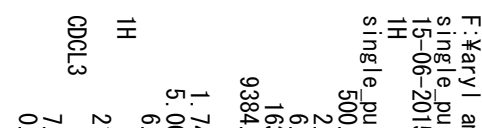

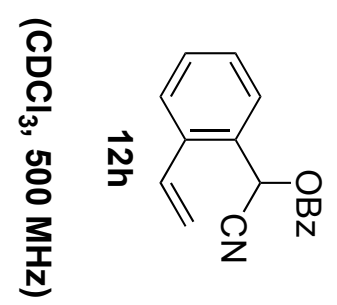
ㅍN요

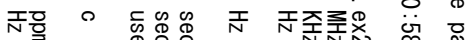



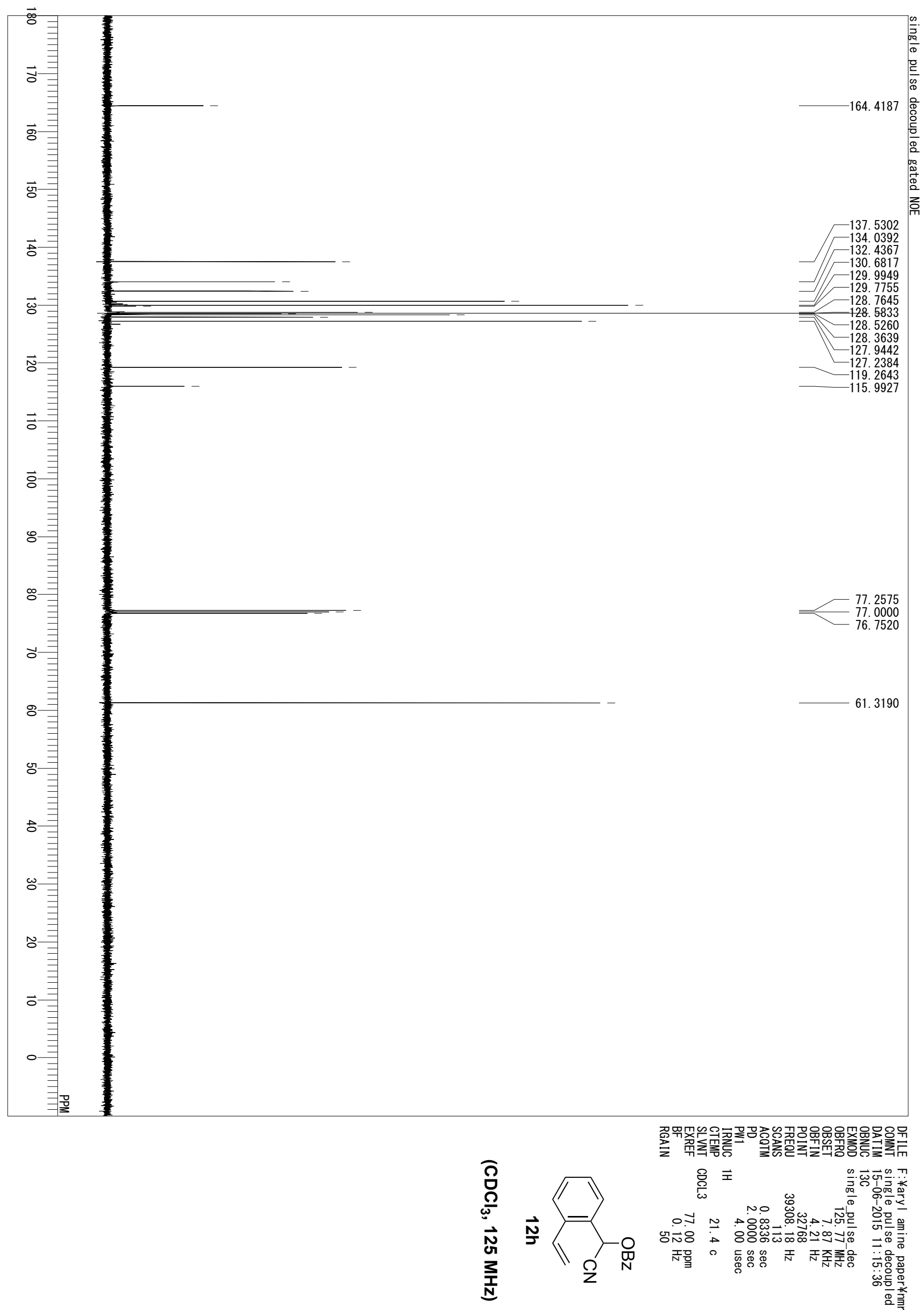

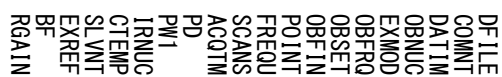

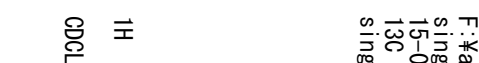

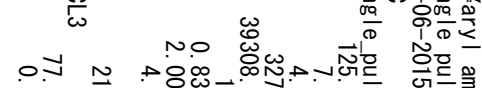

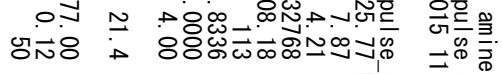

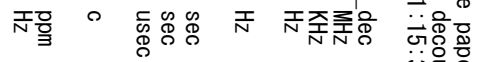

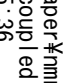




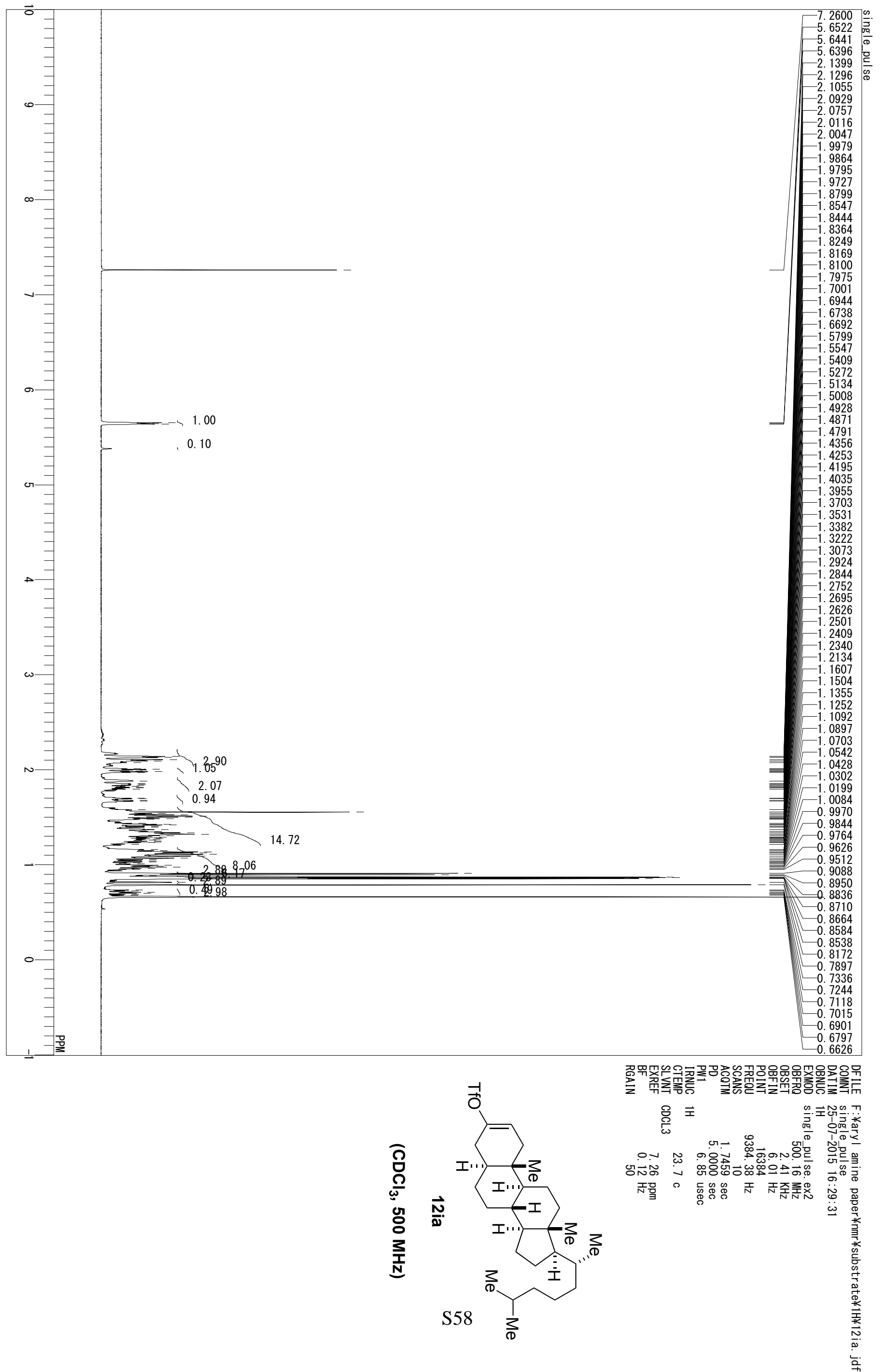




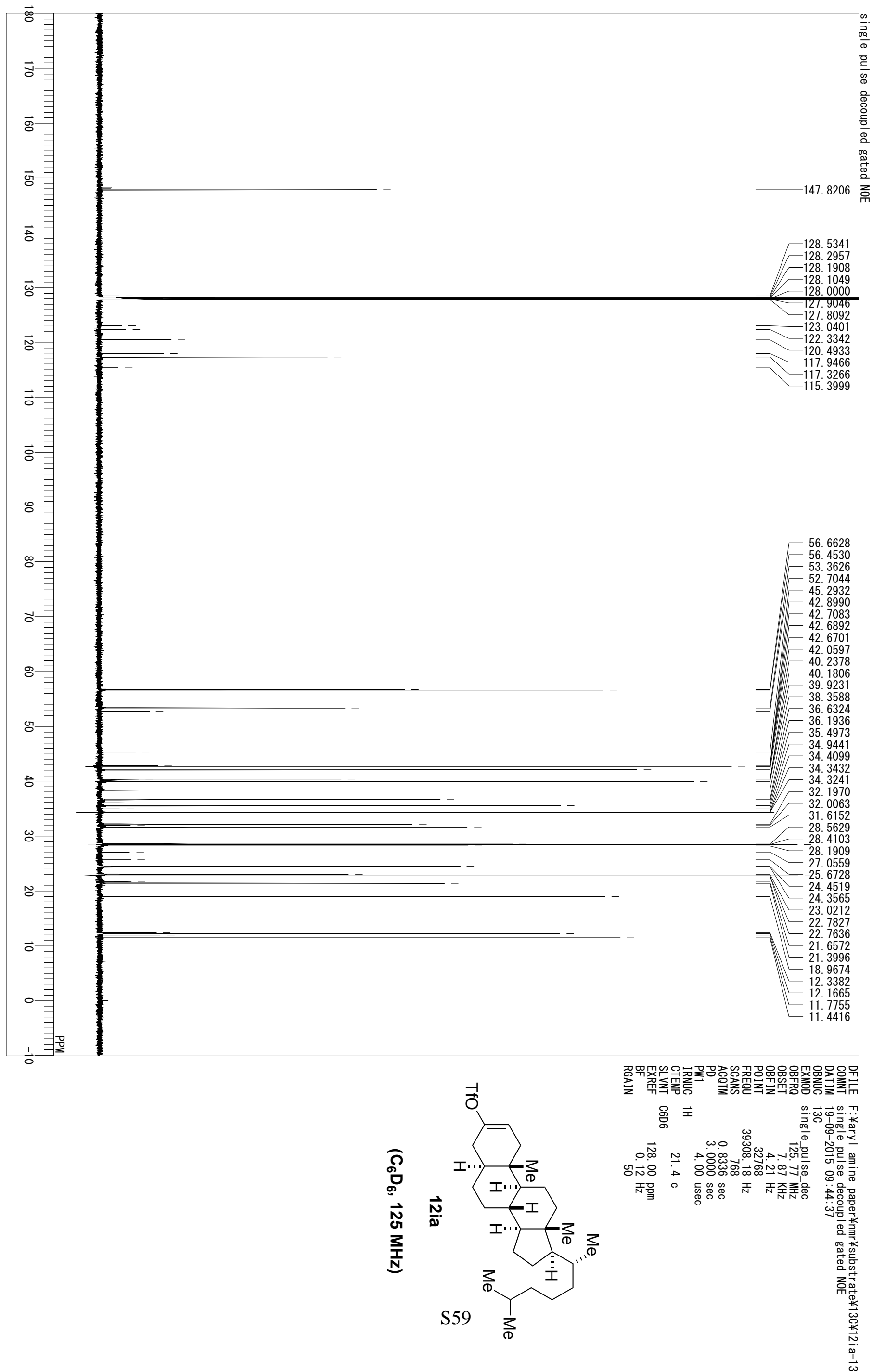



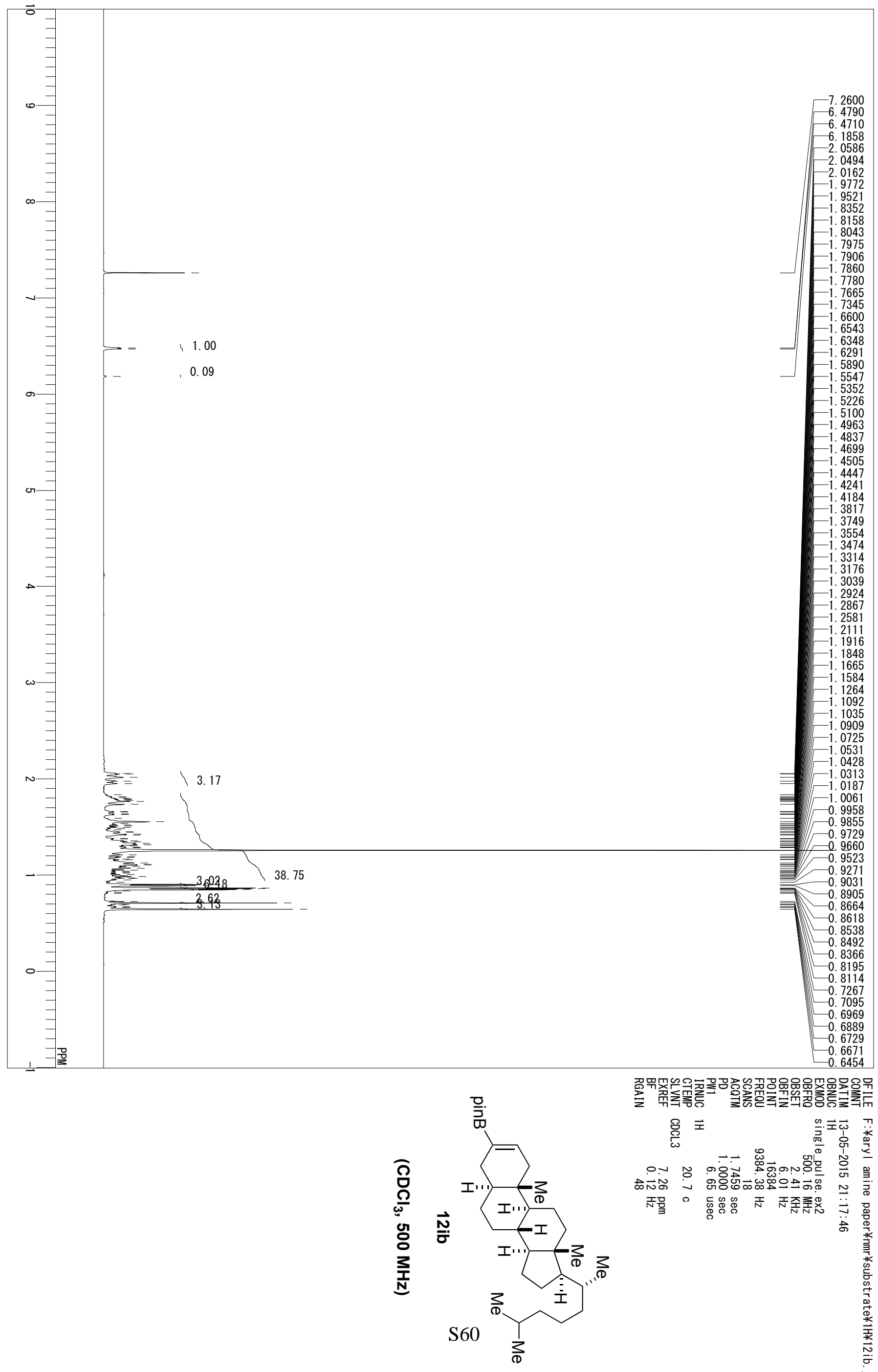

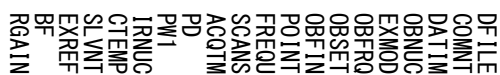

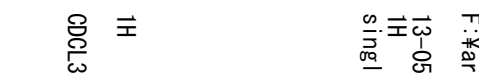

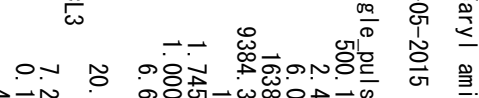
চN

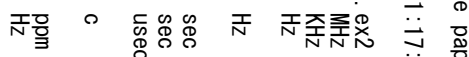
के 


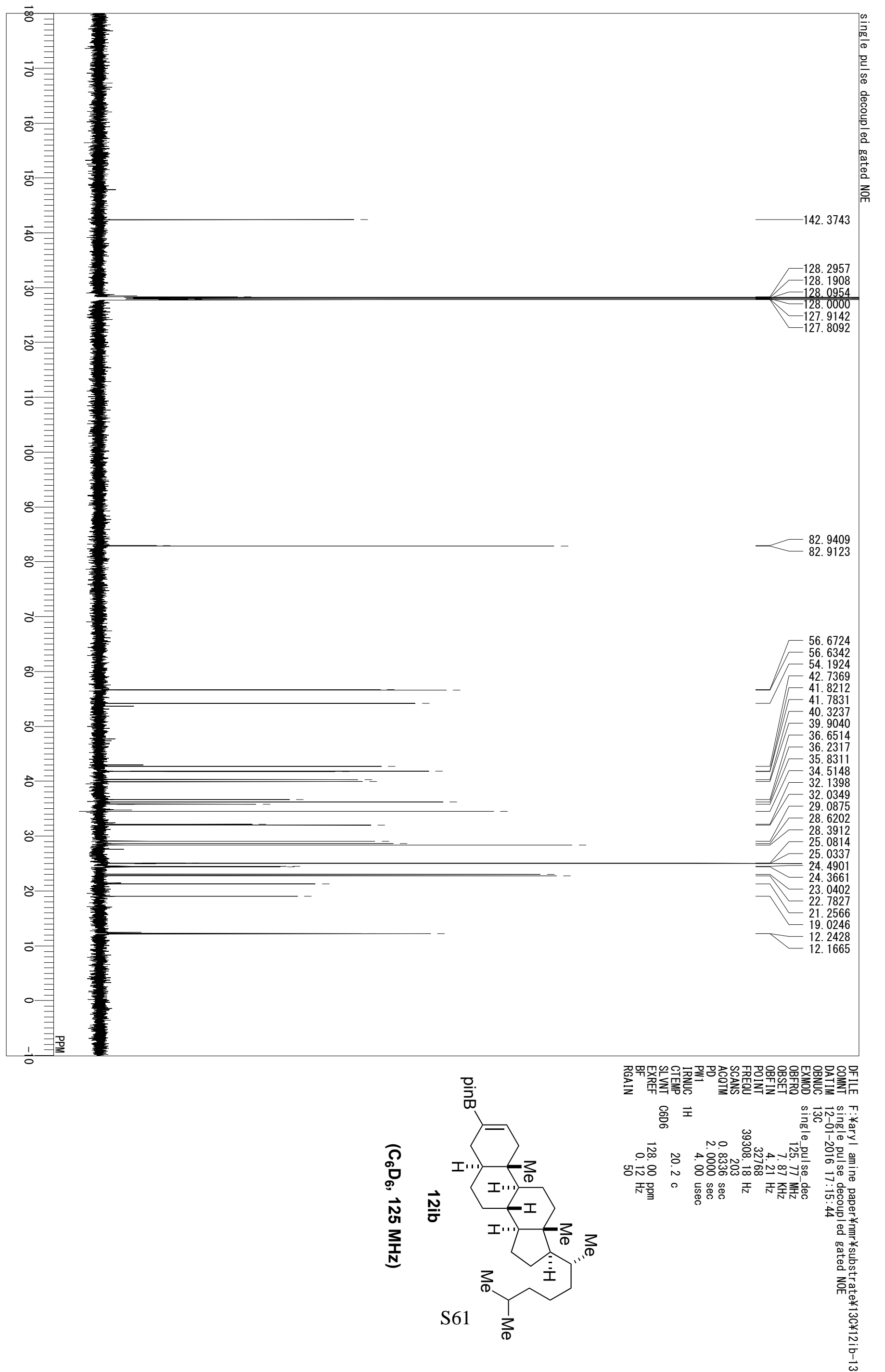




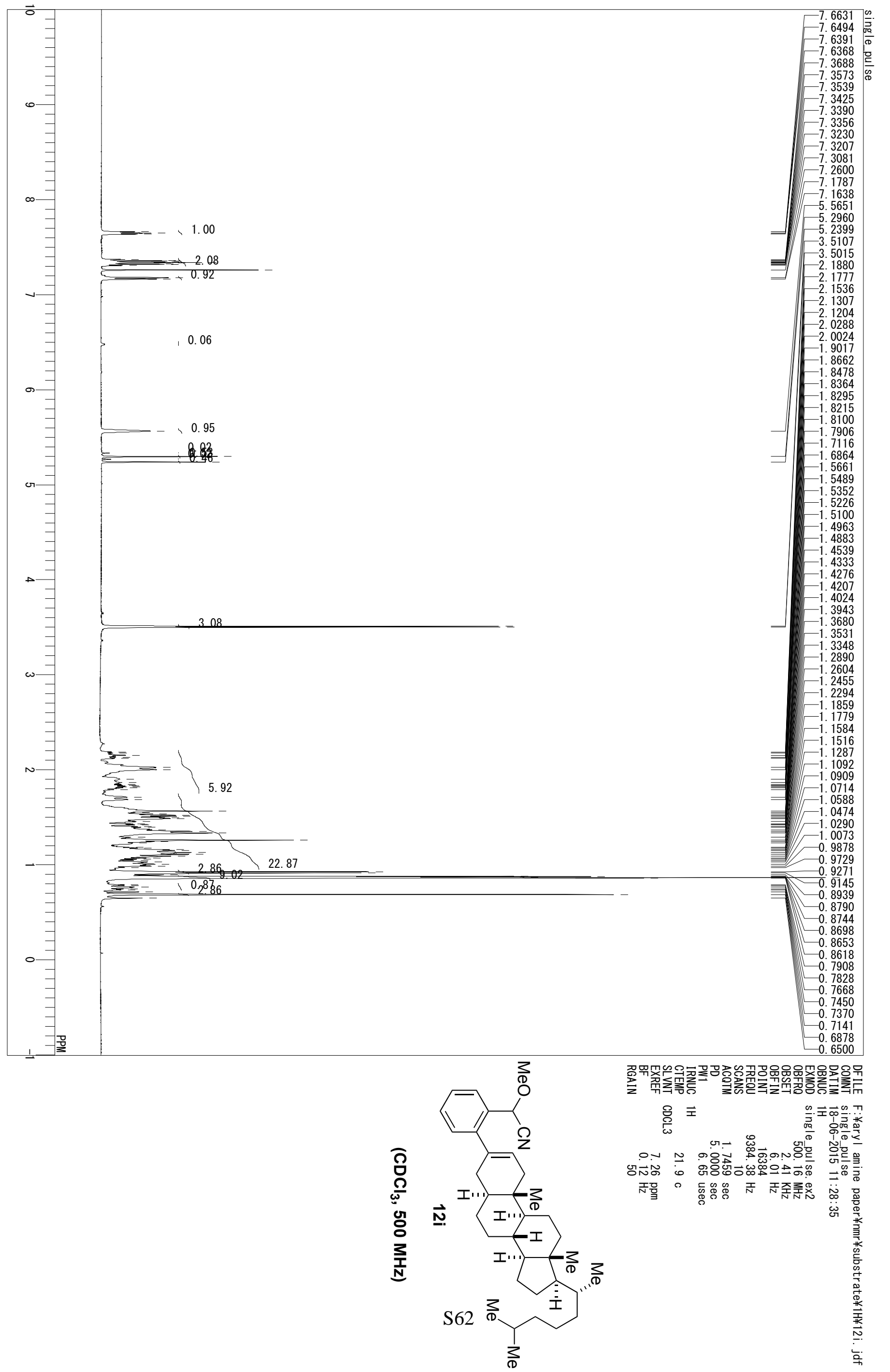



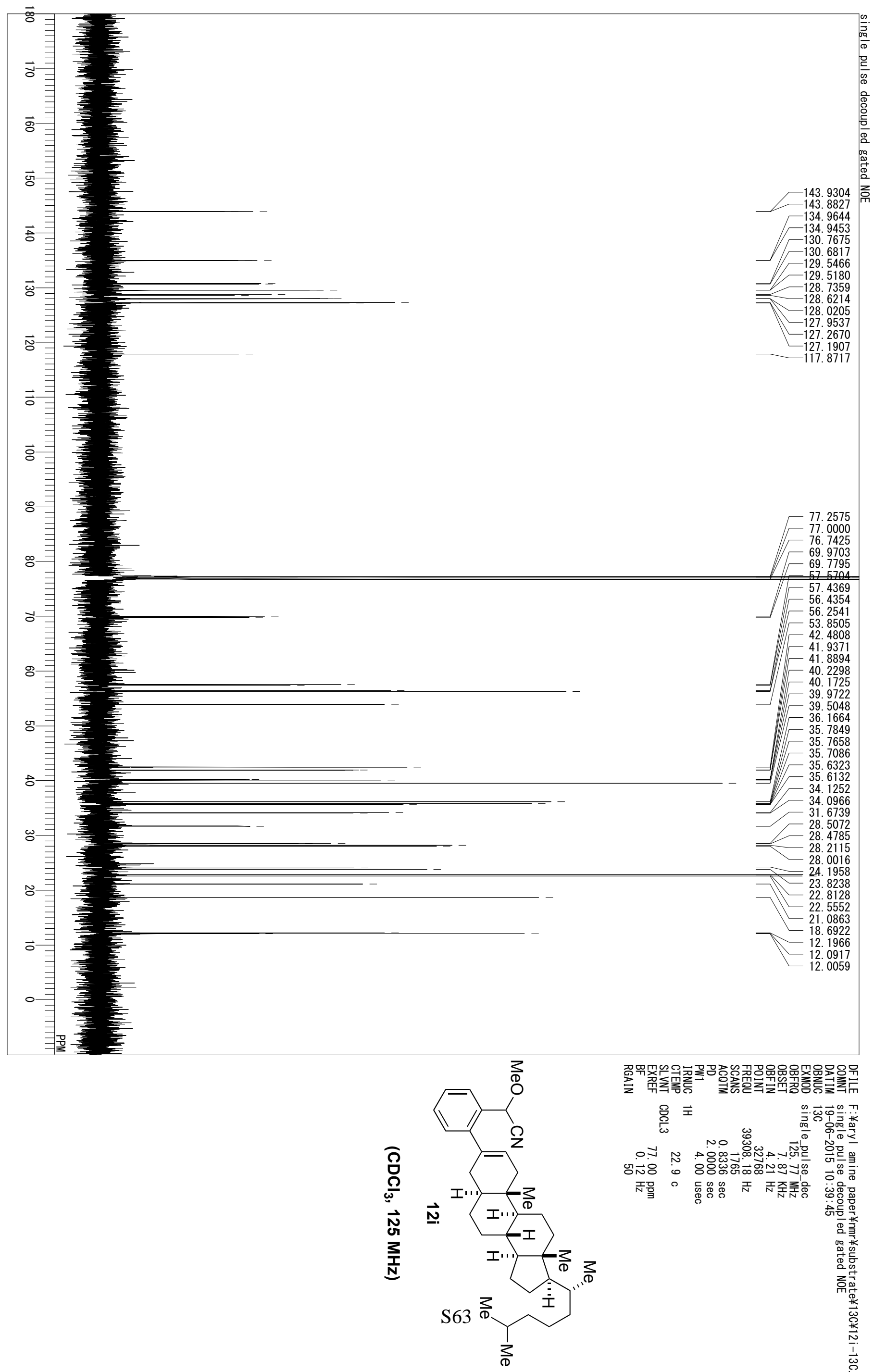

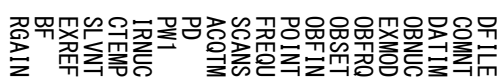
总

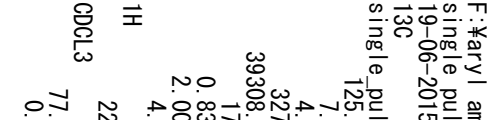

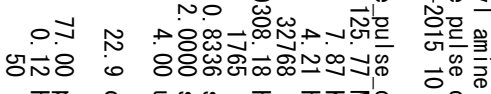

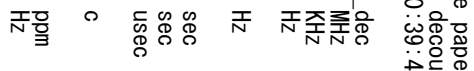

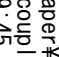




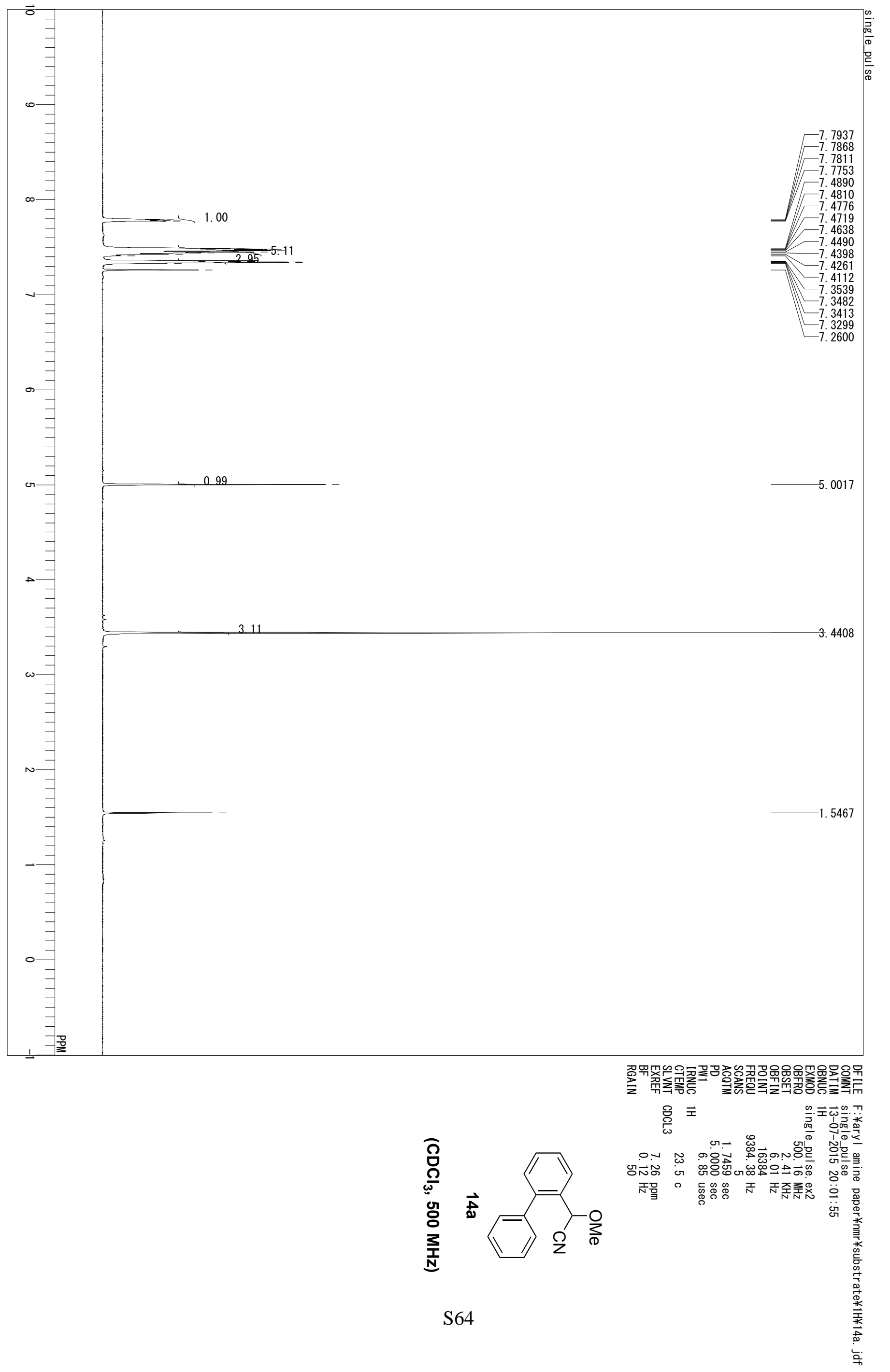




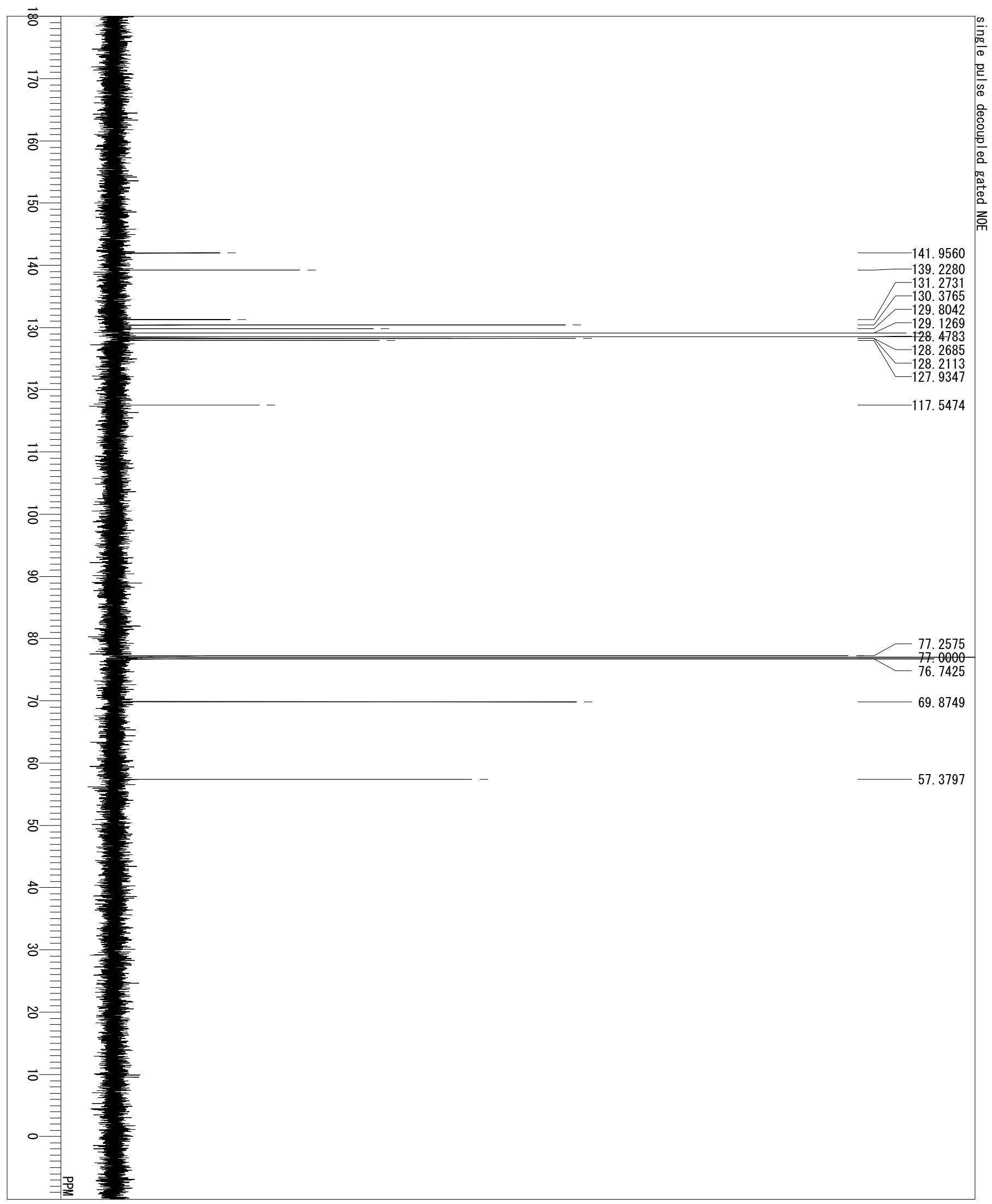

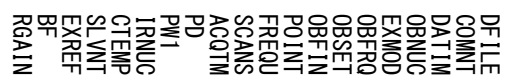
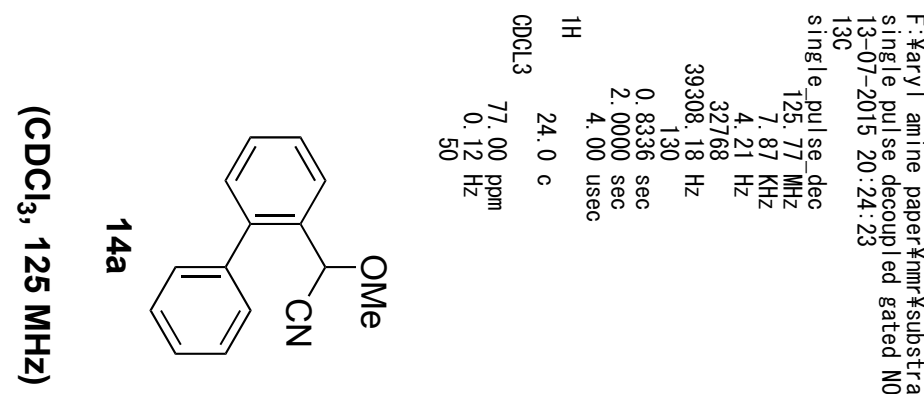
O* N No

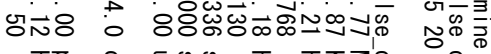

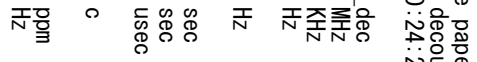




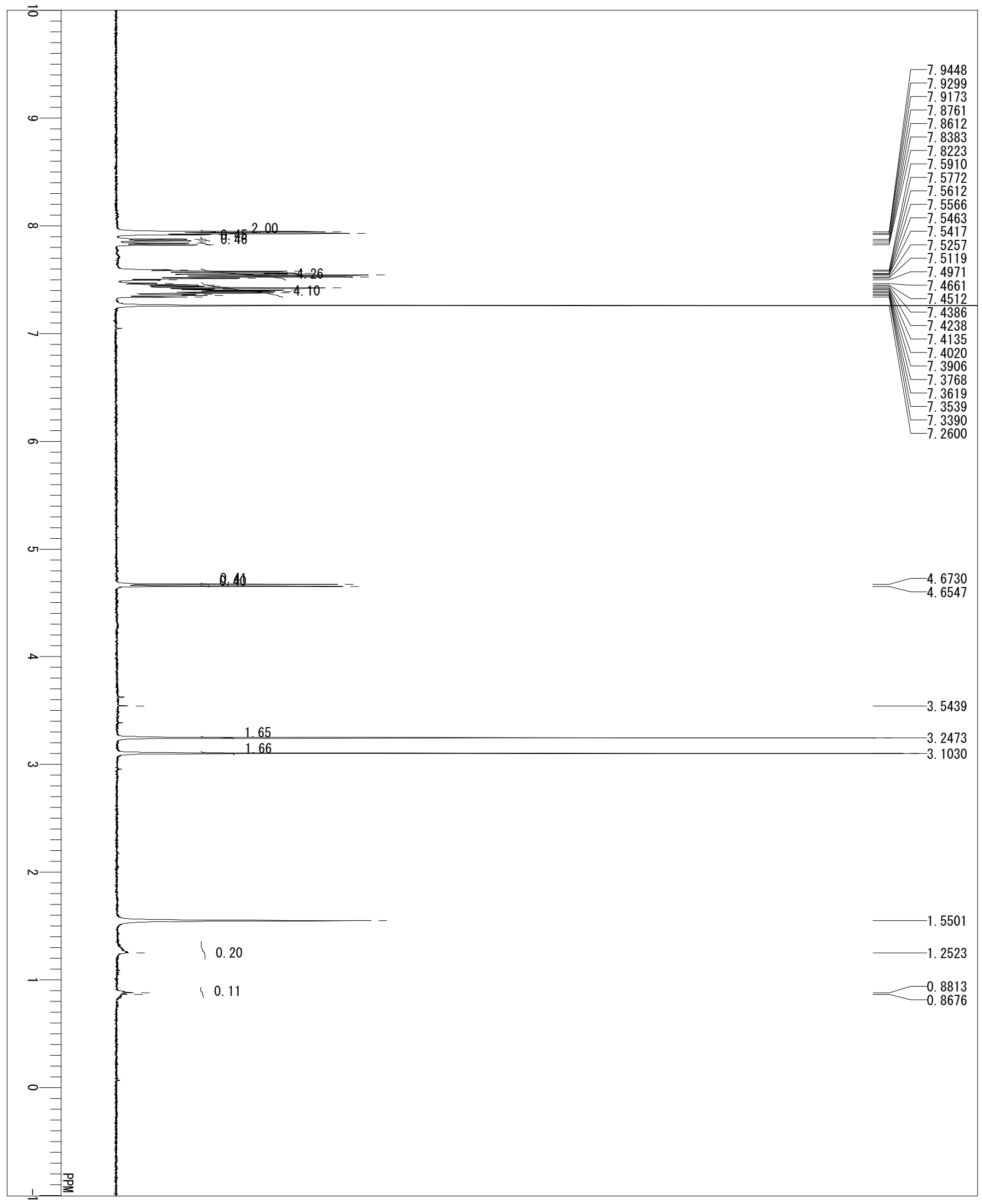

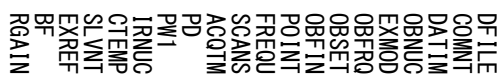

总

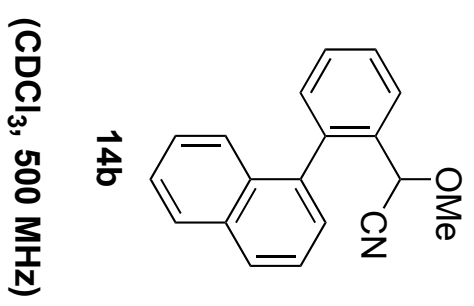

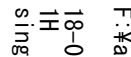

ON $\overrightarrow{0}$ क्षे

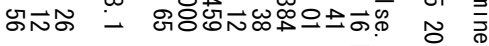

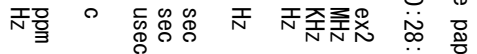




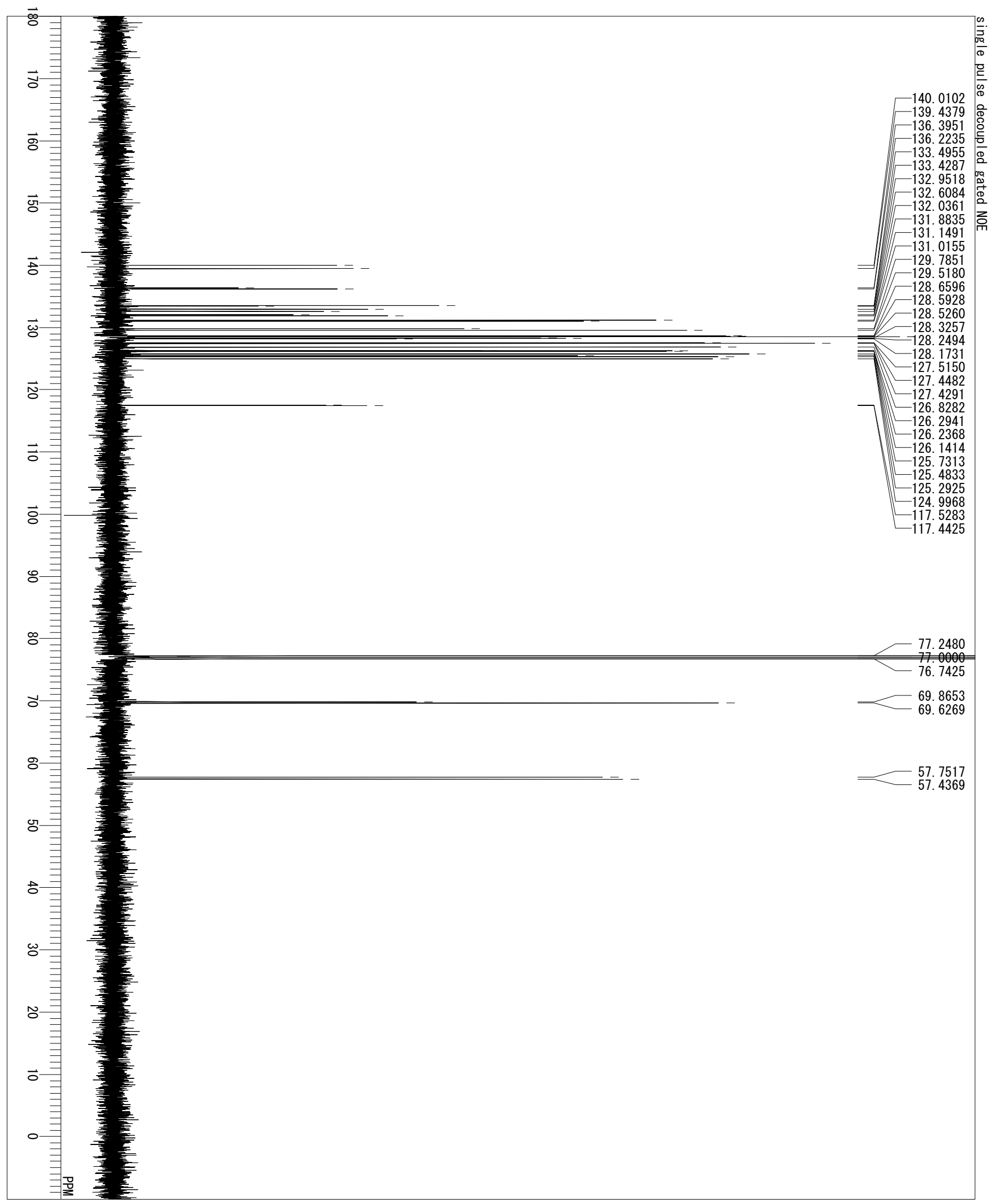

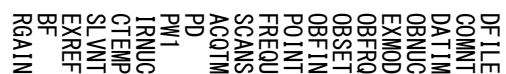
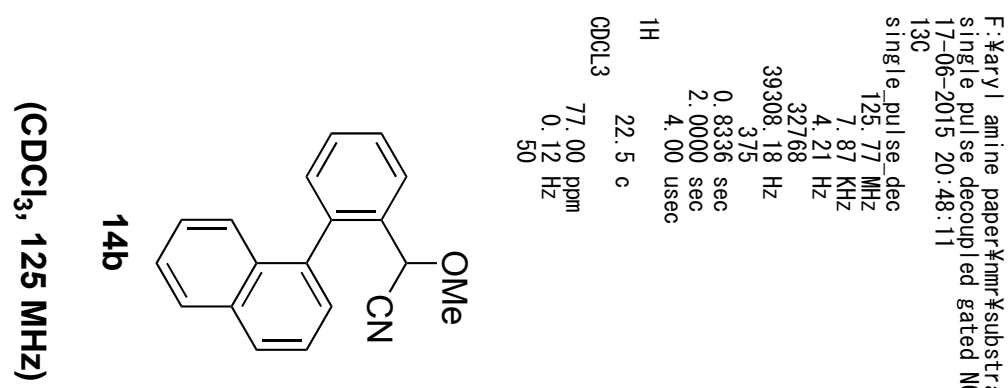

ON N

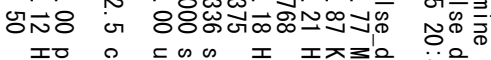

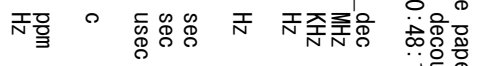




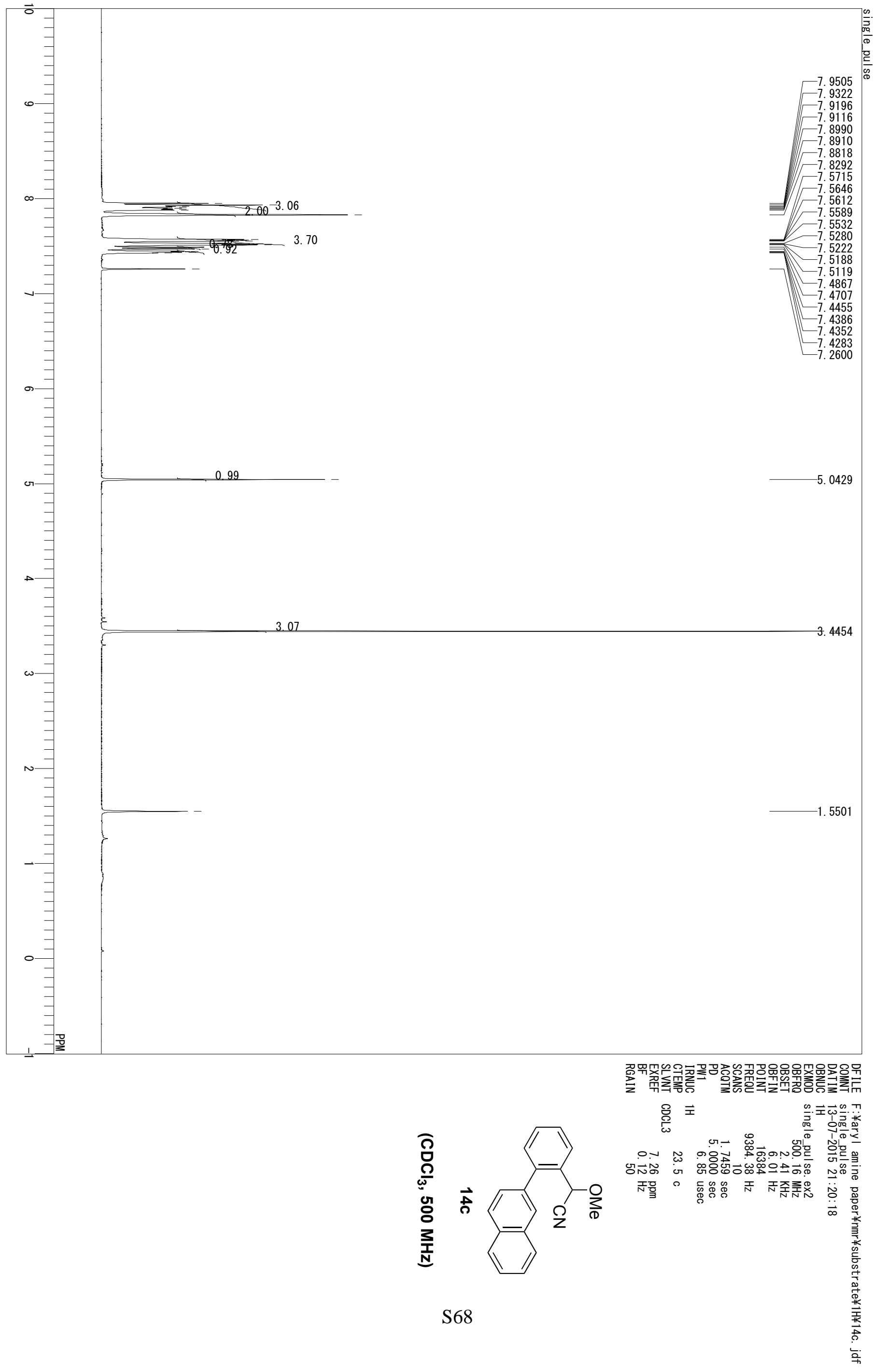




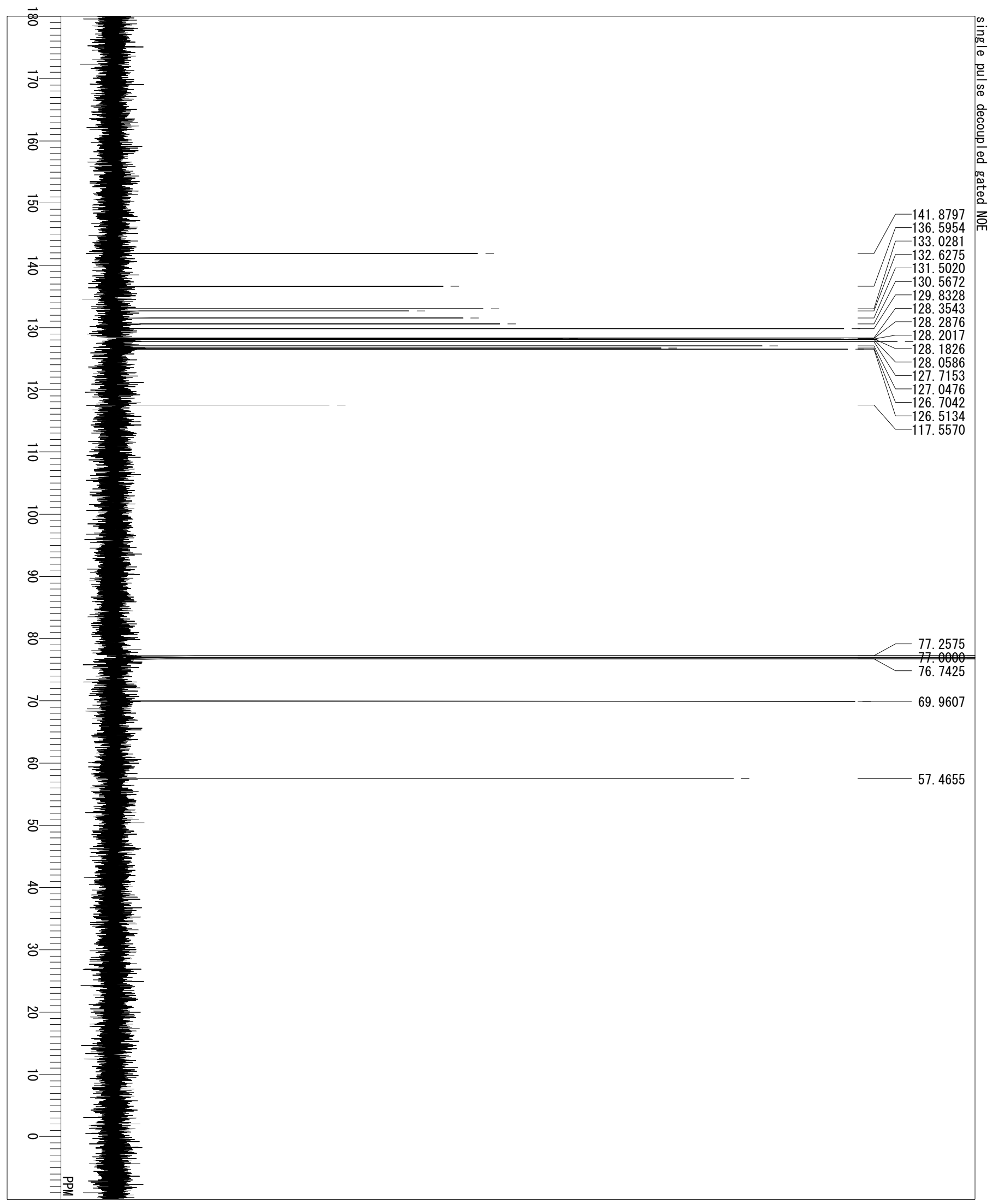

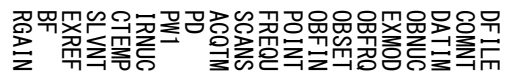

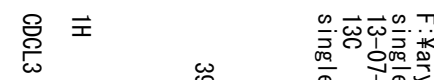

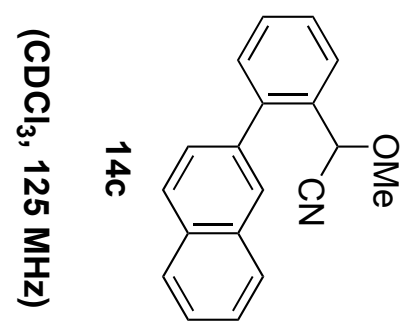

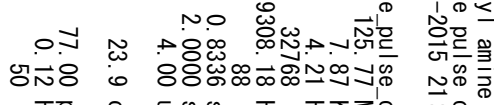

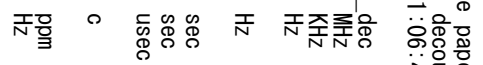




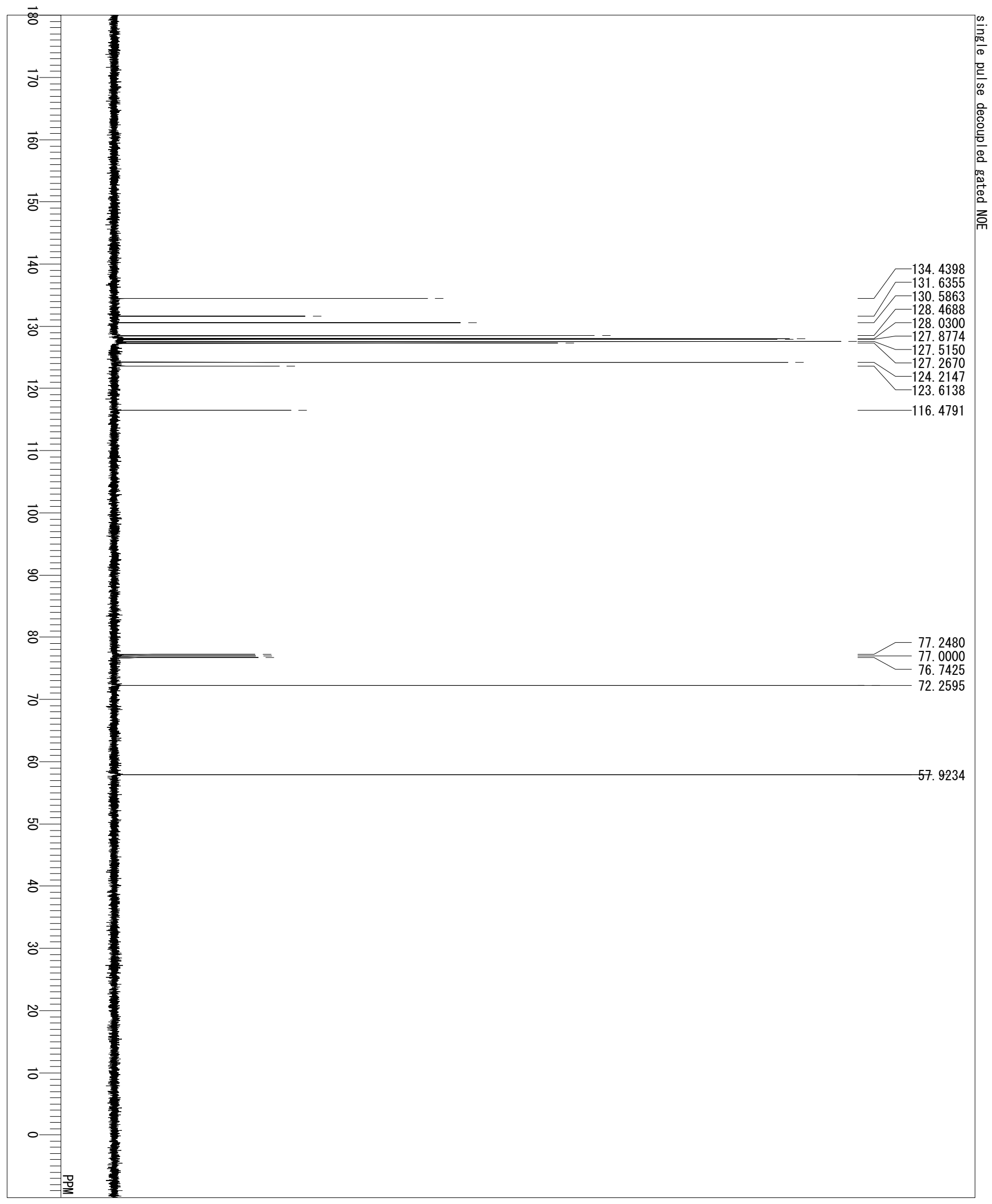

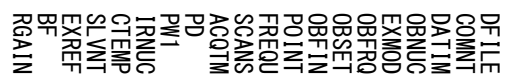

흔

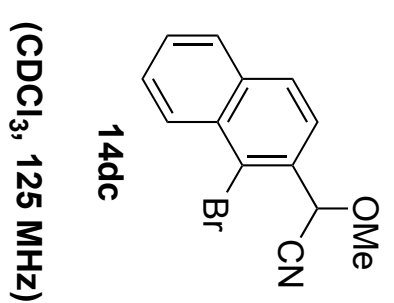

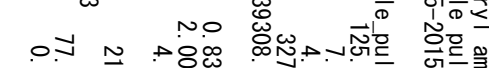
जis:

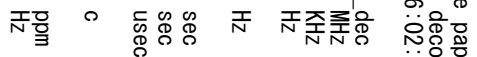

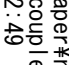




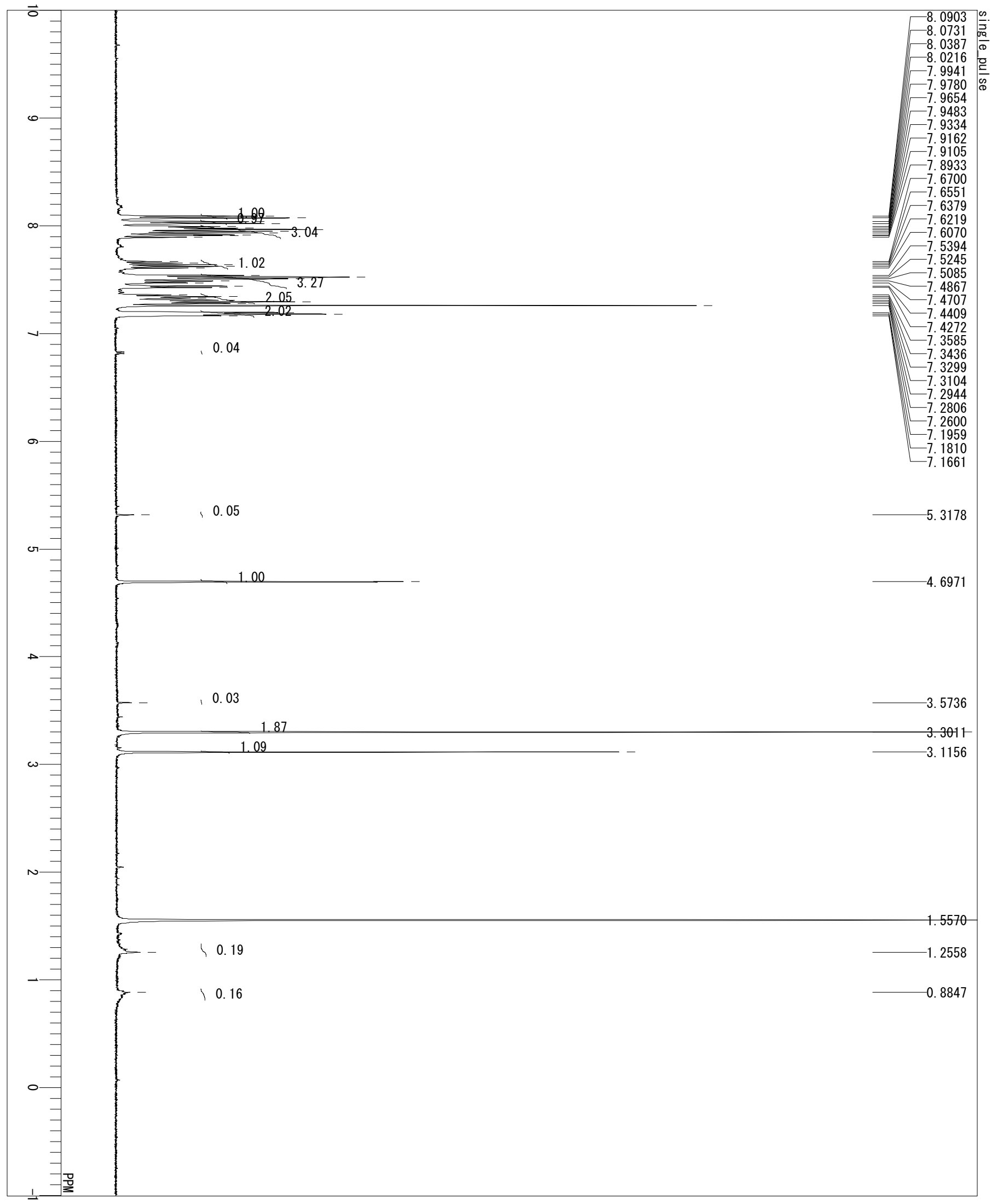

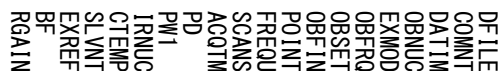
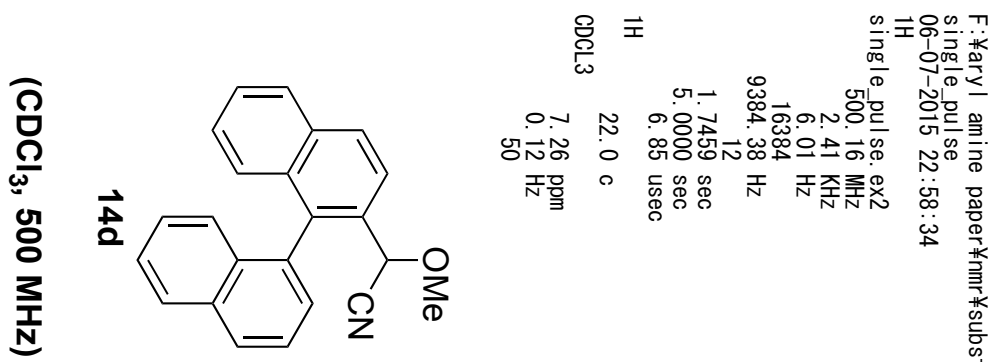

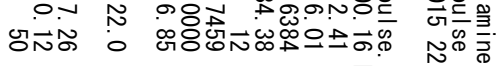

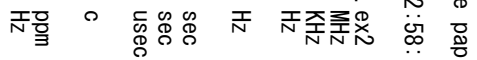




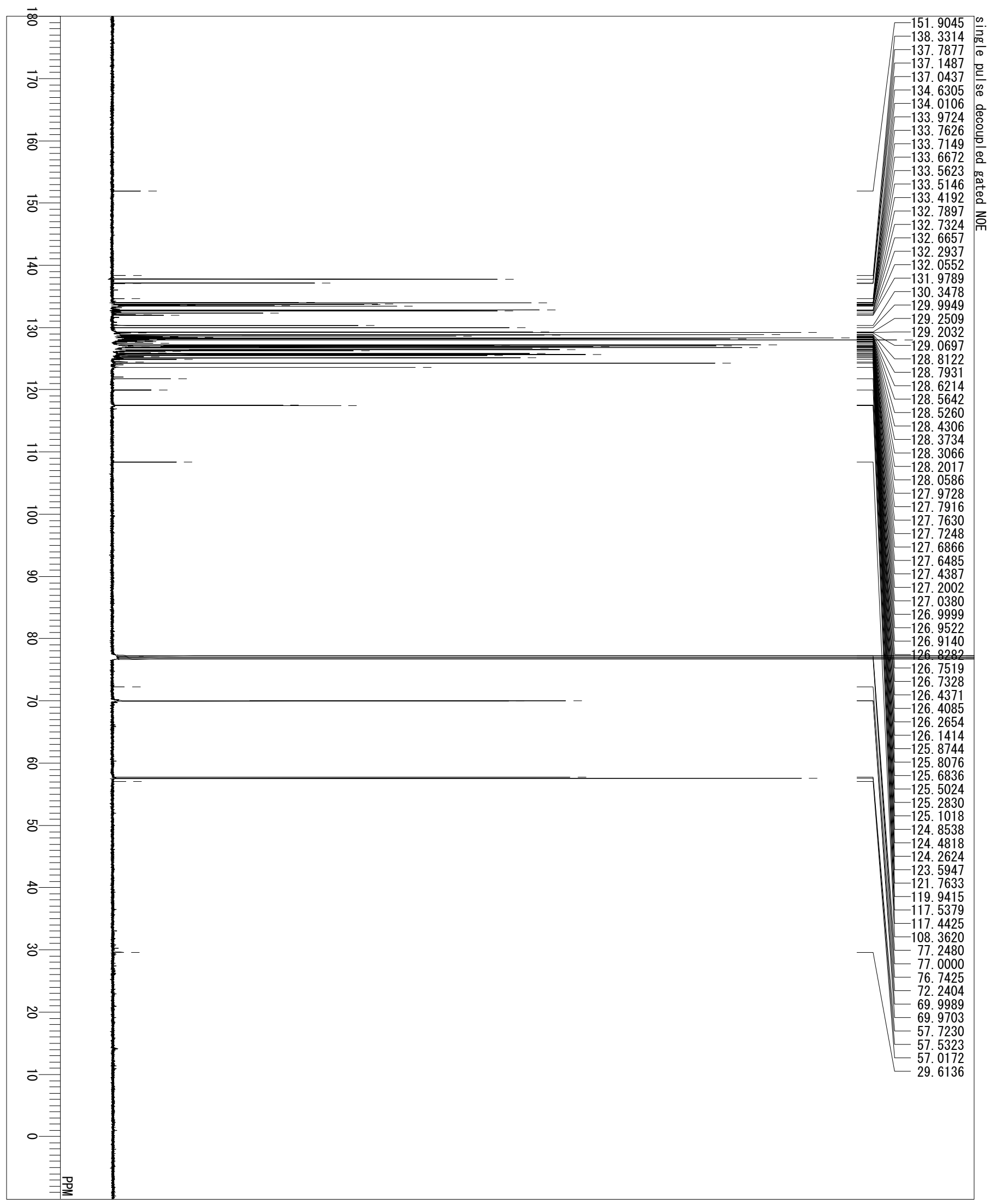

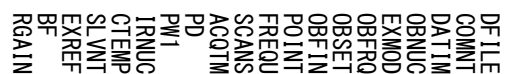

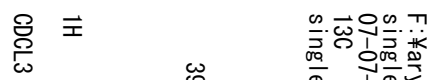

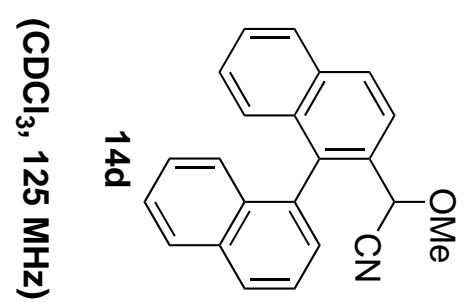

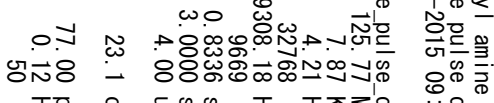

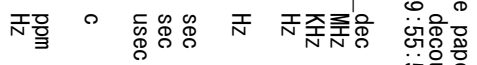

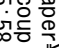




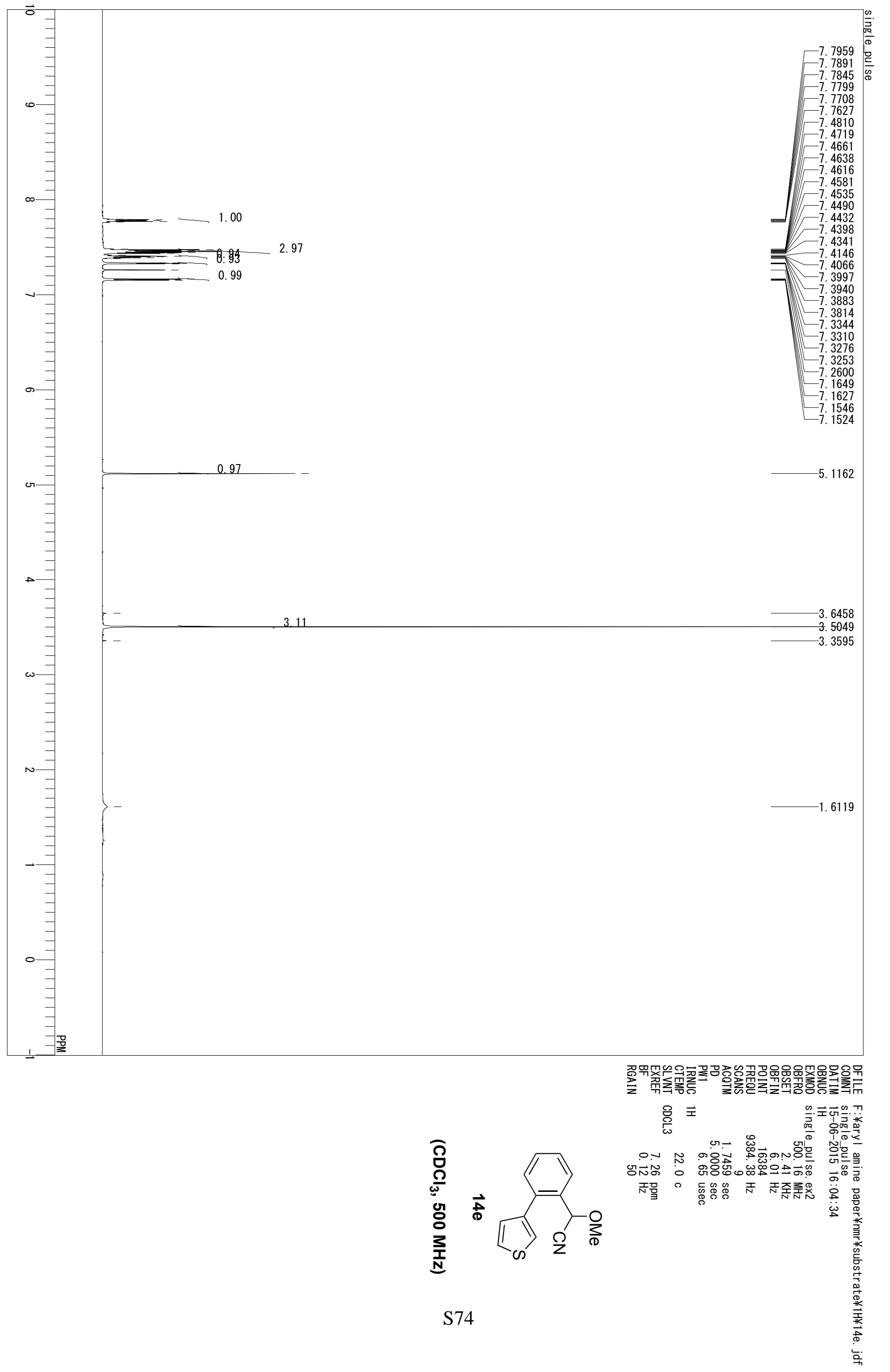




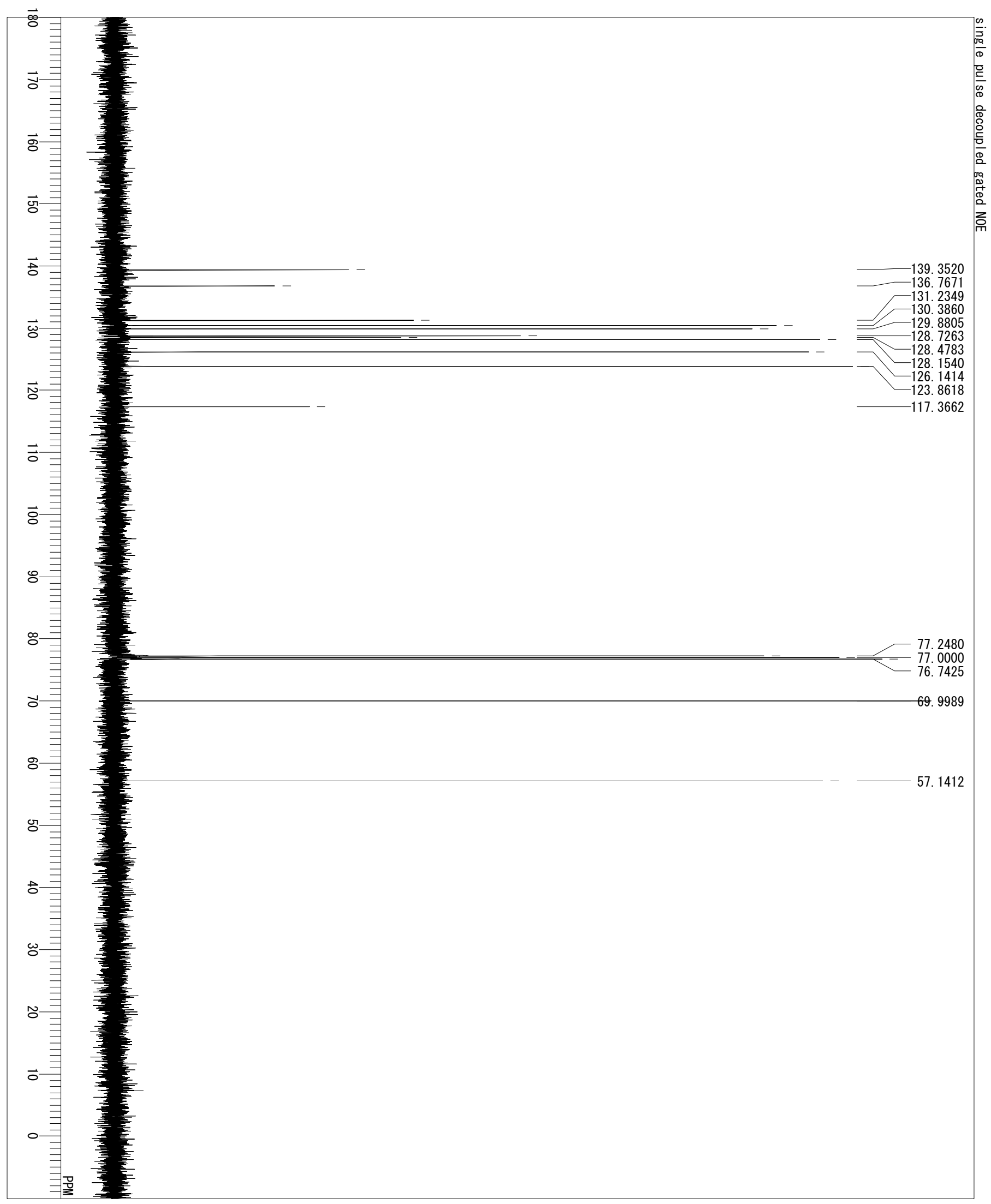

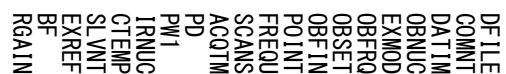

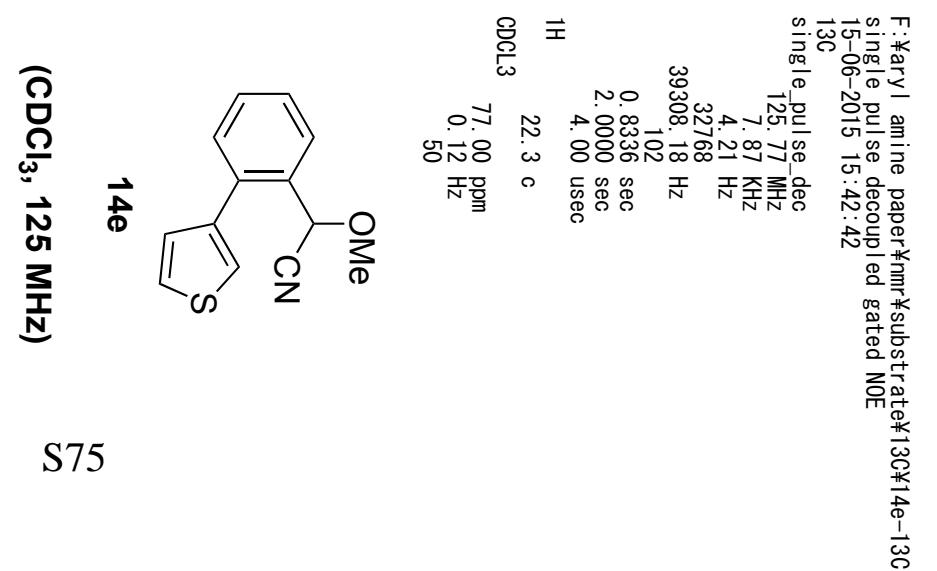




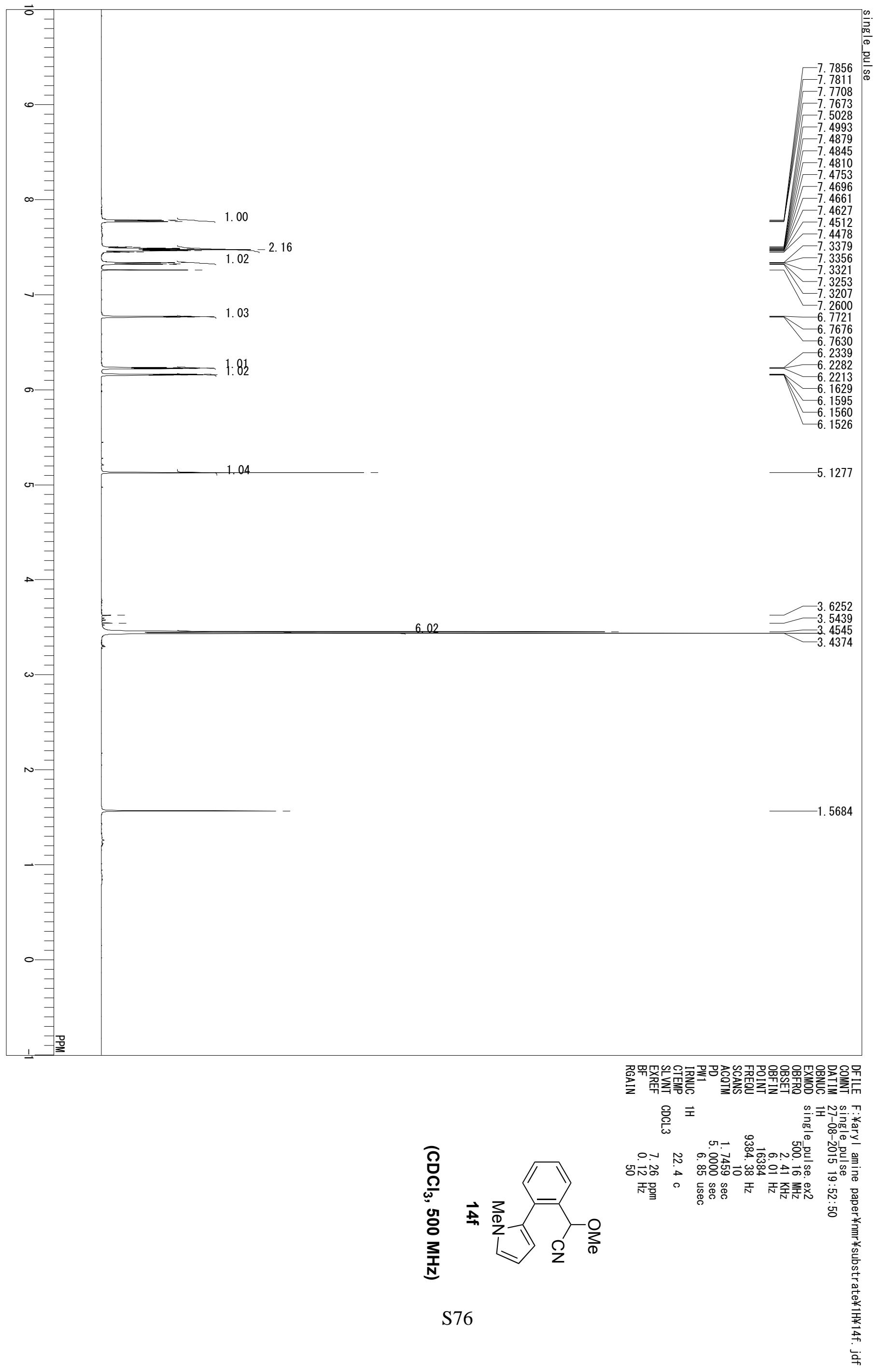




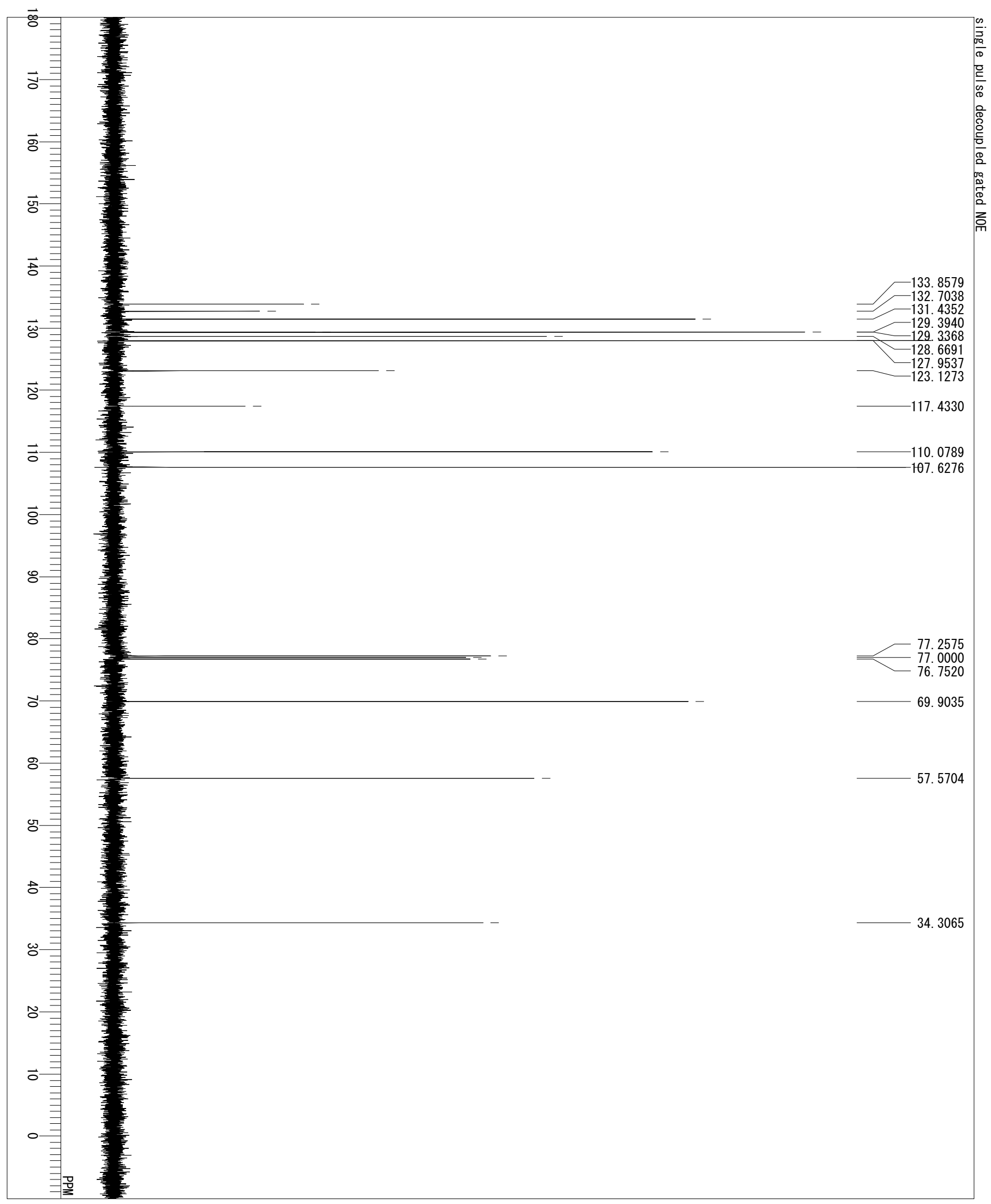

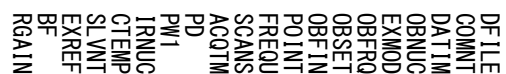
总现)

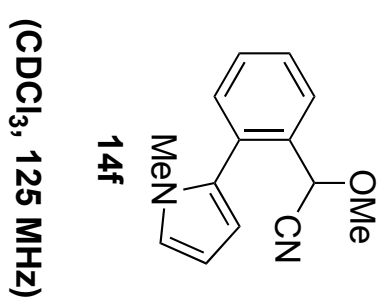

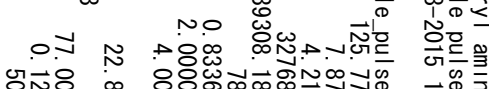
건응 0 에 폰믕 0 동 


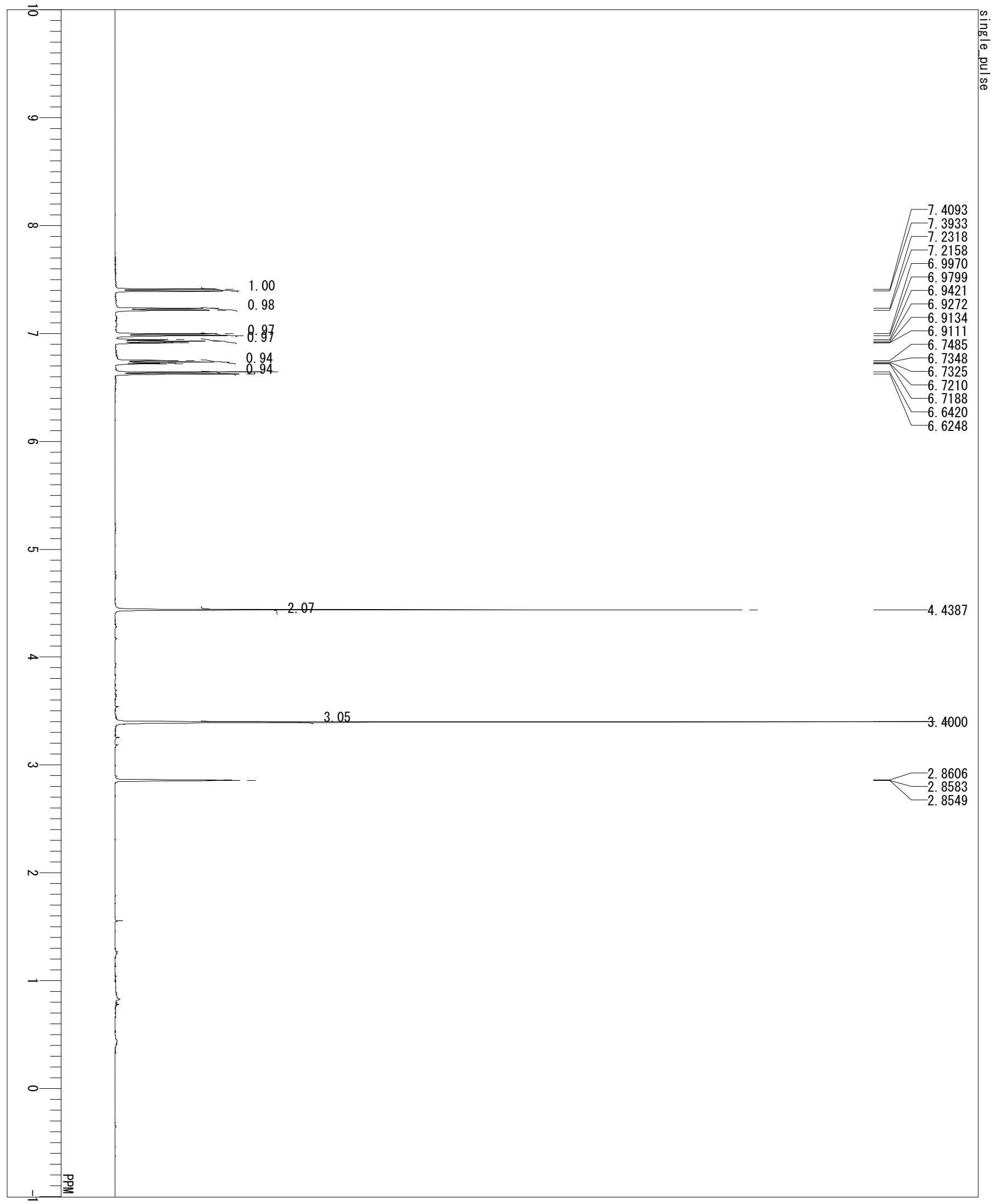

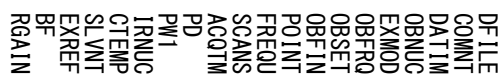

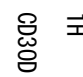

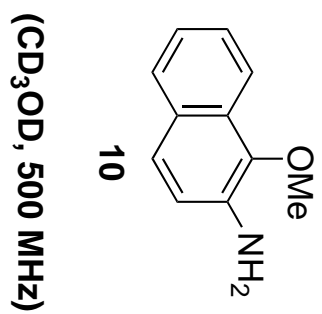

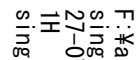
ow N

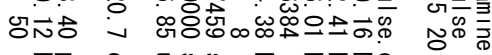
점을 


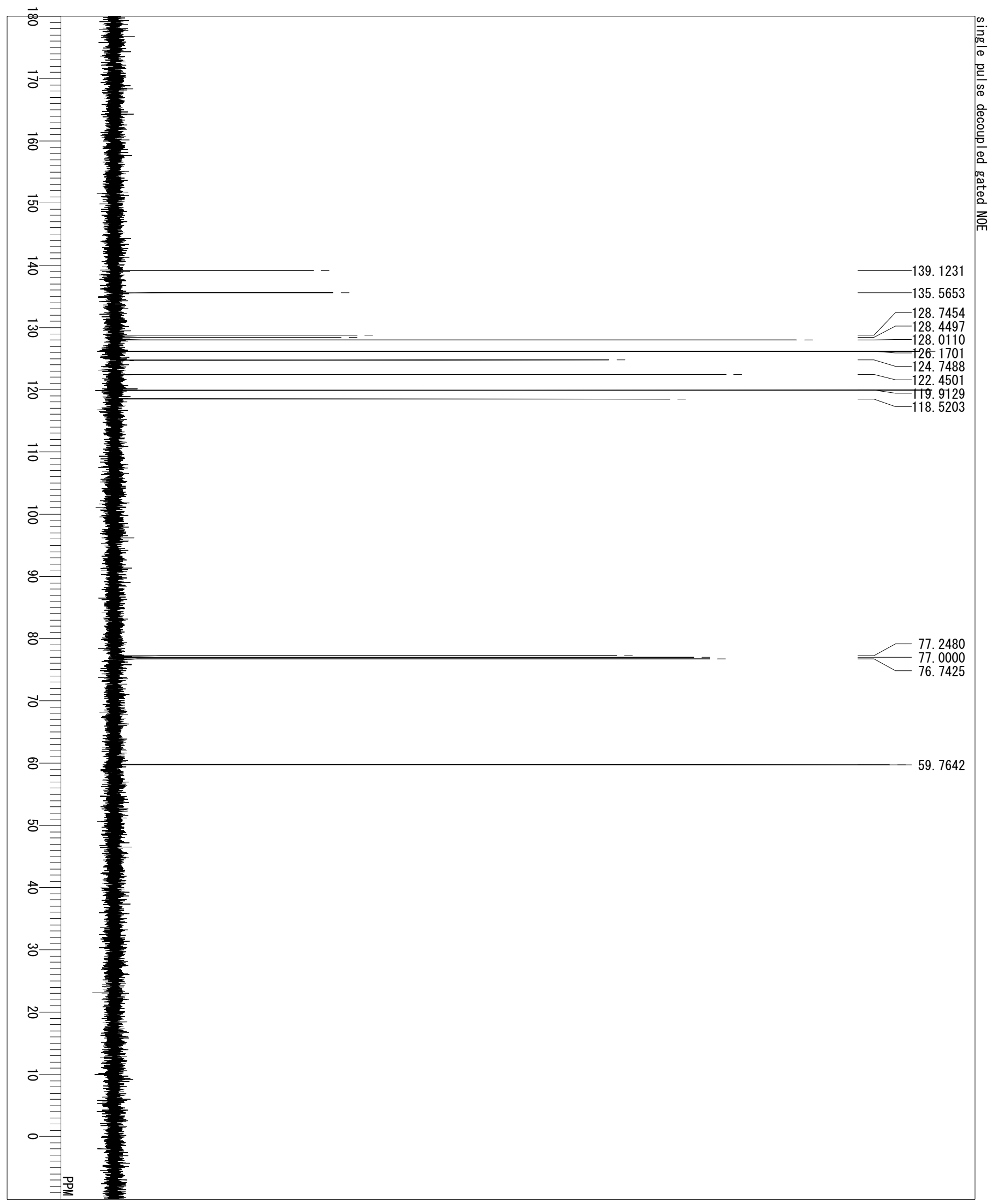

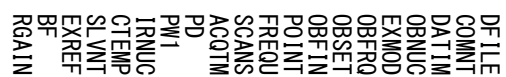

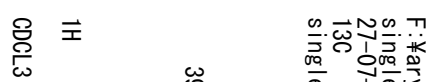

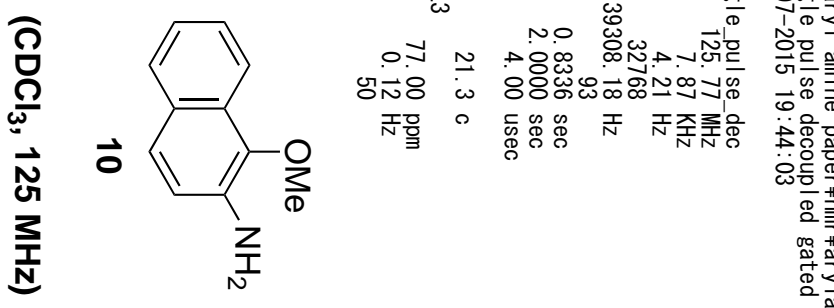

S79 


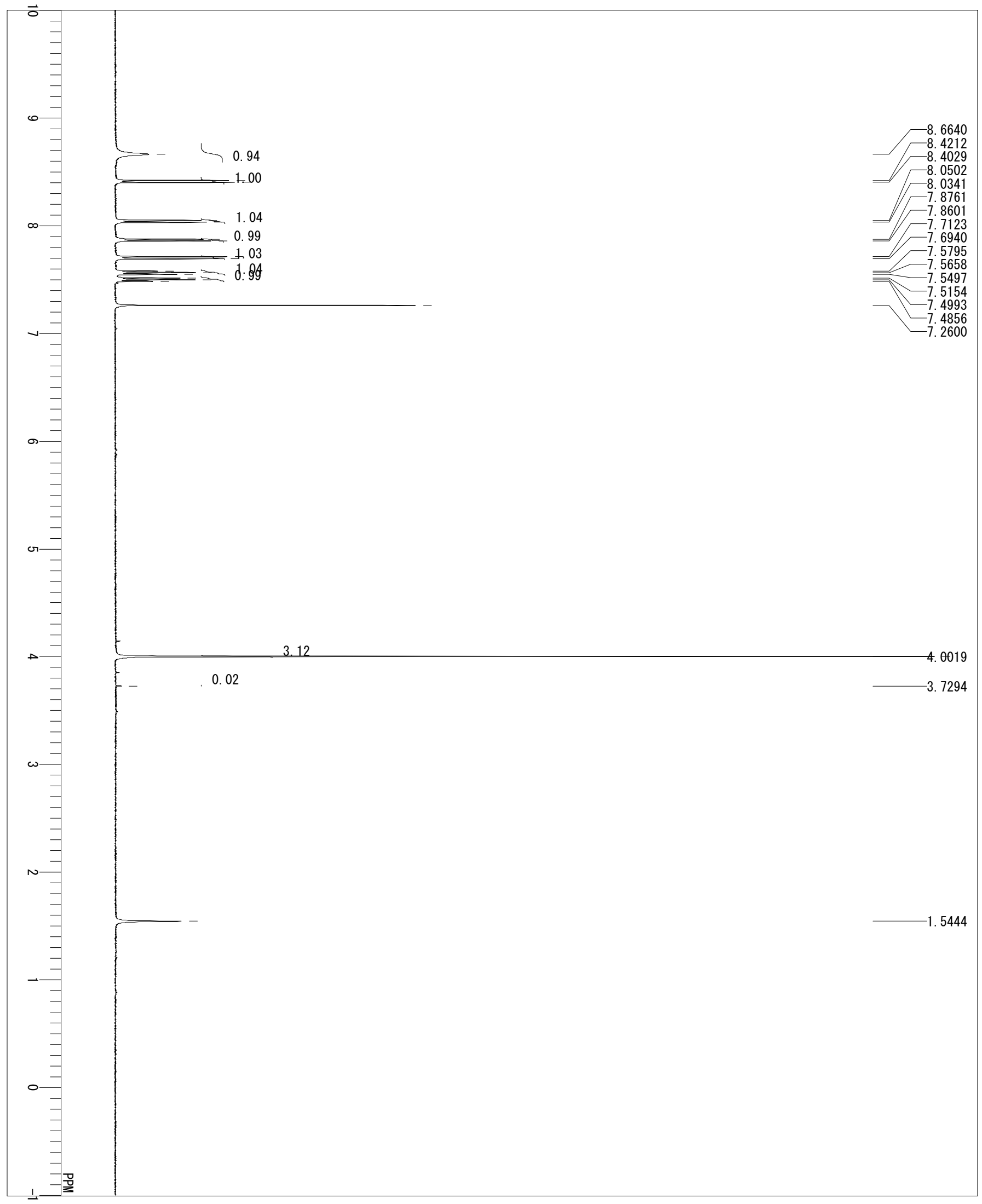

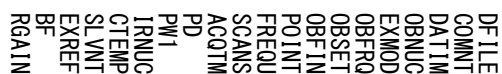

舀

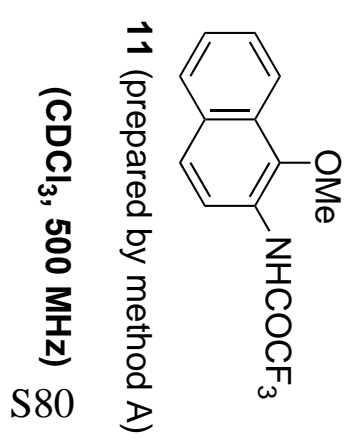

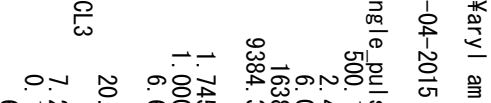

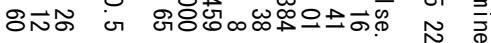

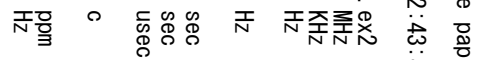




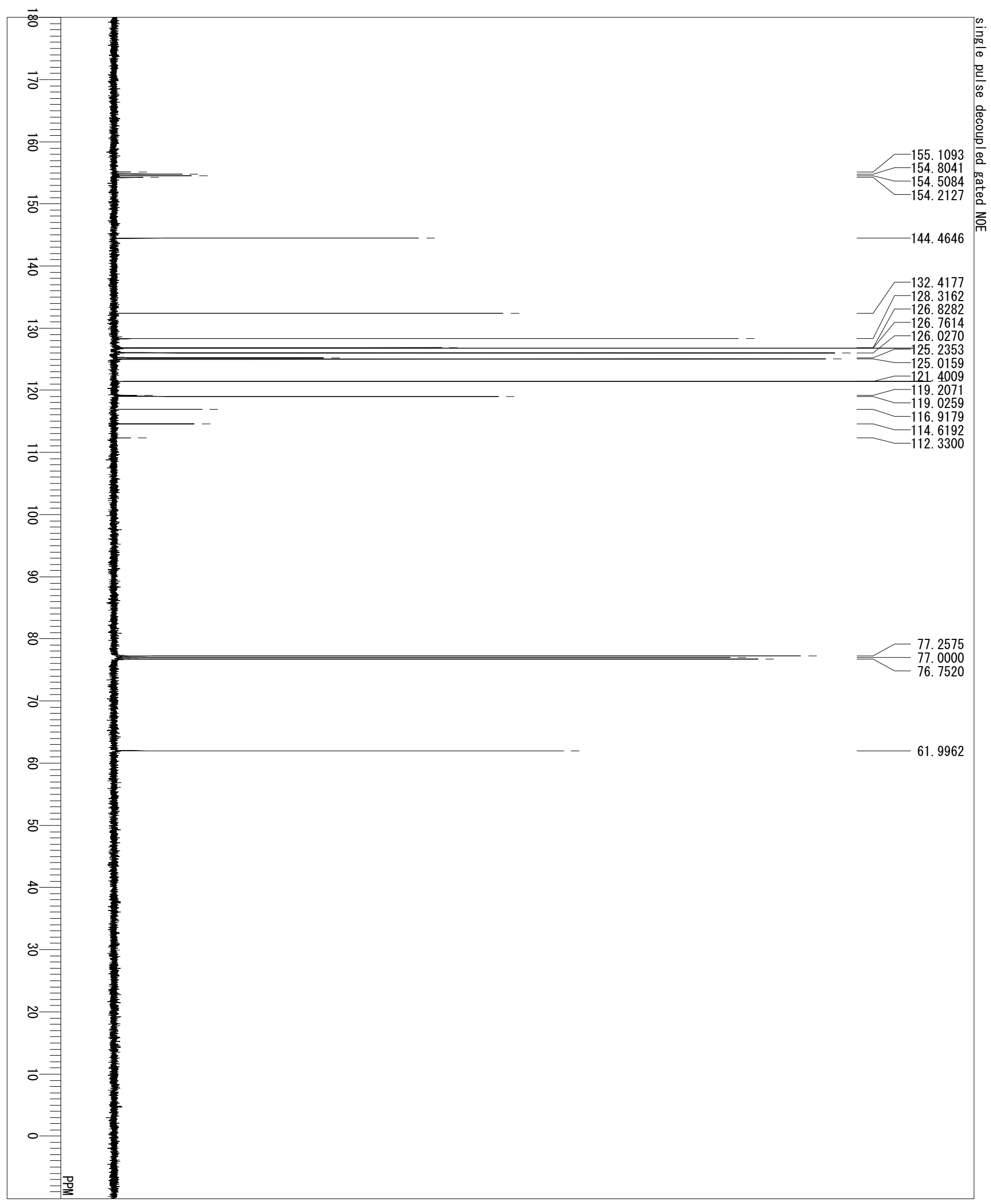

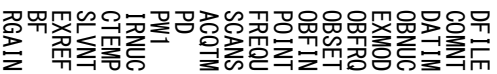

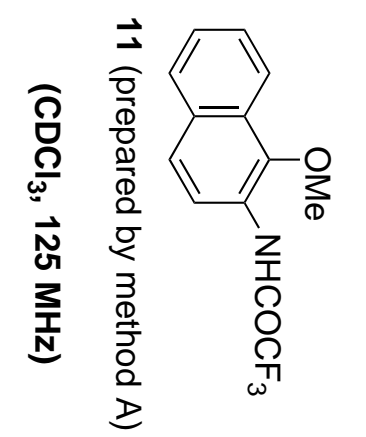

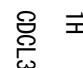

wo

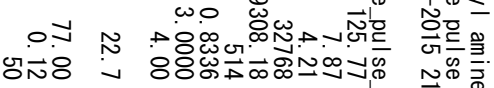

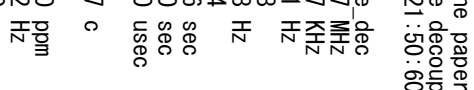




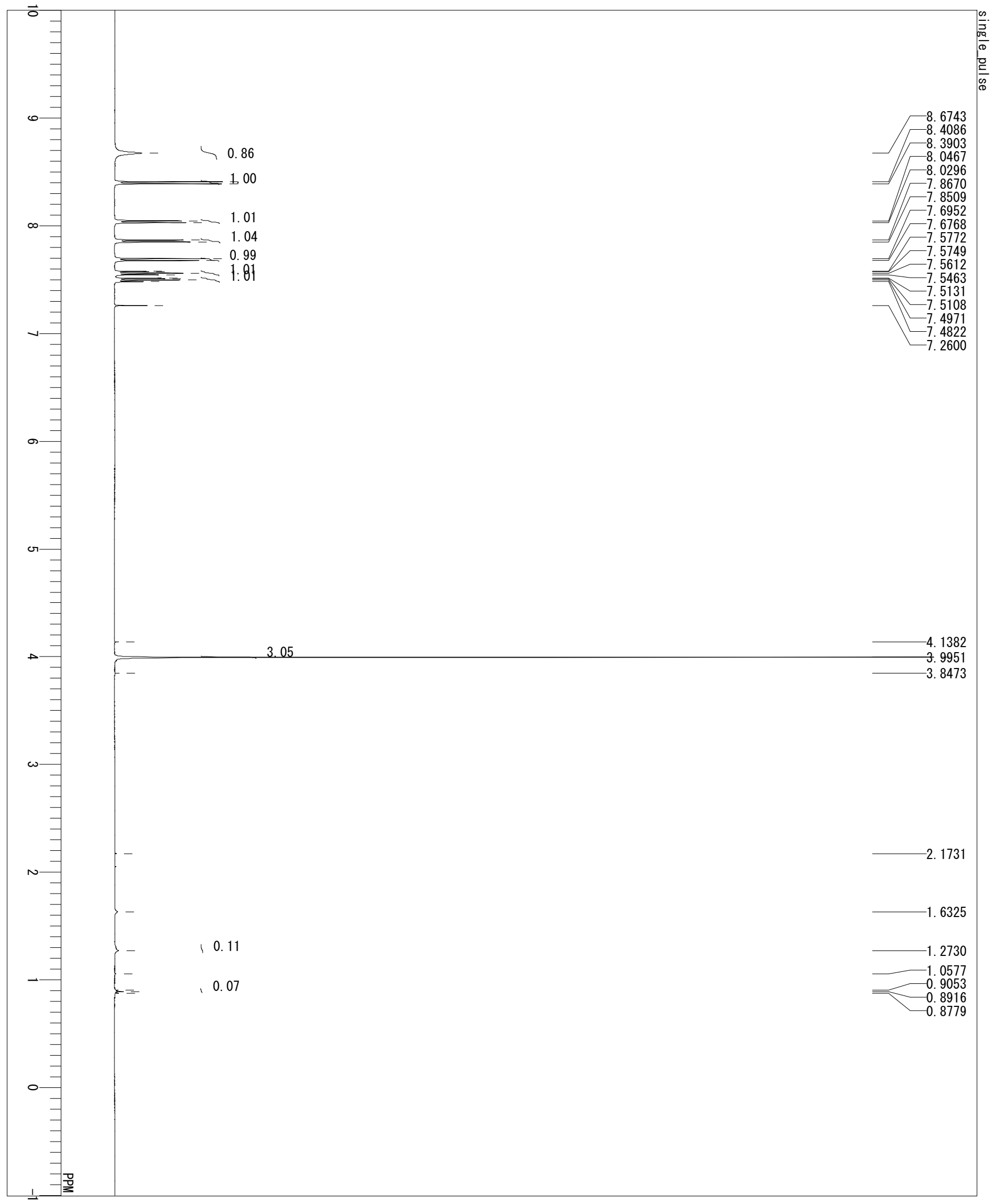

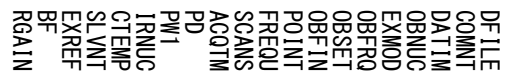

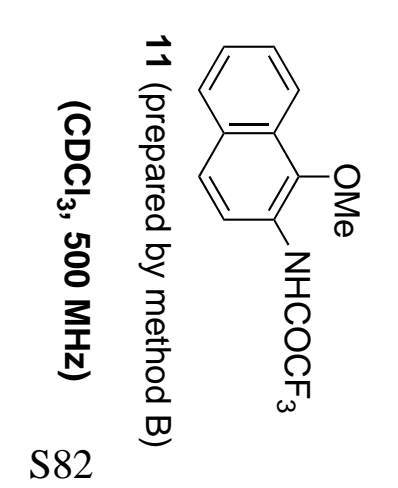

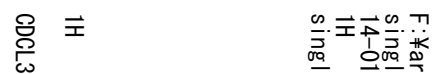

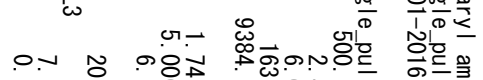
JNN i

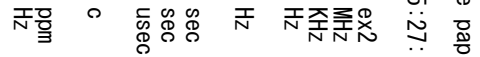
$\frac{\pi}{\mathbb{N}}$ 


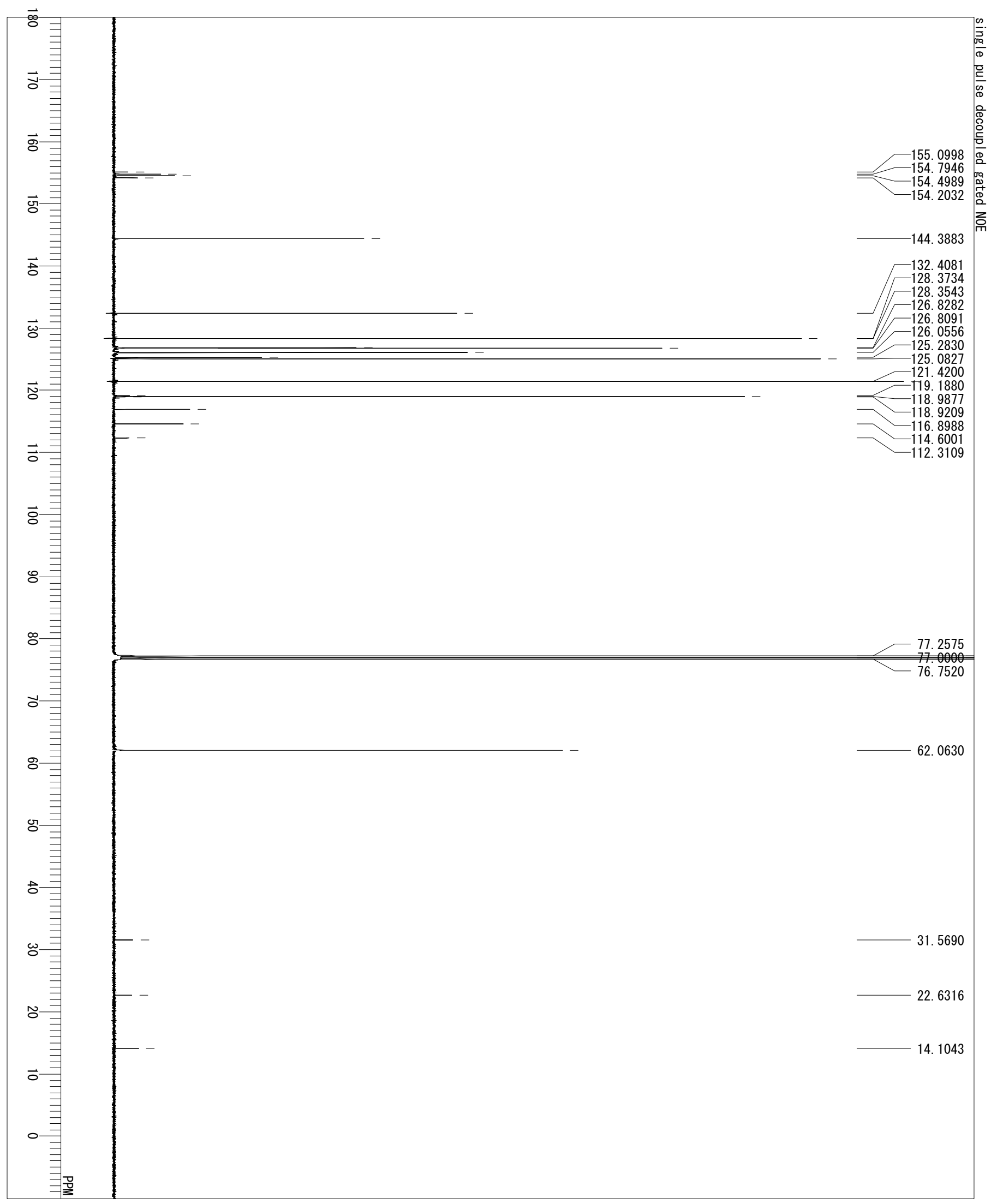

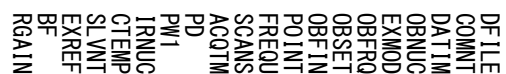

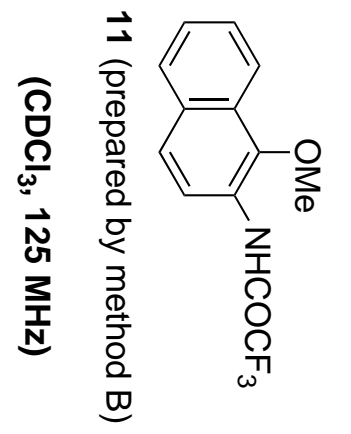

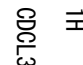

$\stackrel{\omega}{\vec{*}} \vec{\omega} \infty \omega$ wo

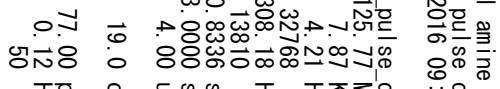

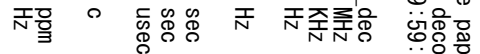
흐응 $\frac{\mathbb{D}}{\mathbb{1}}$ 

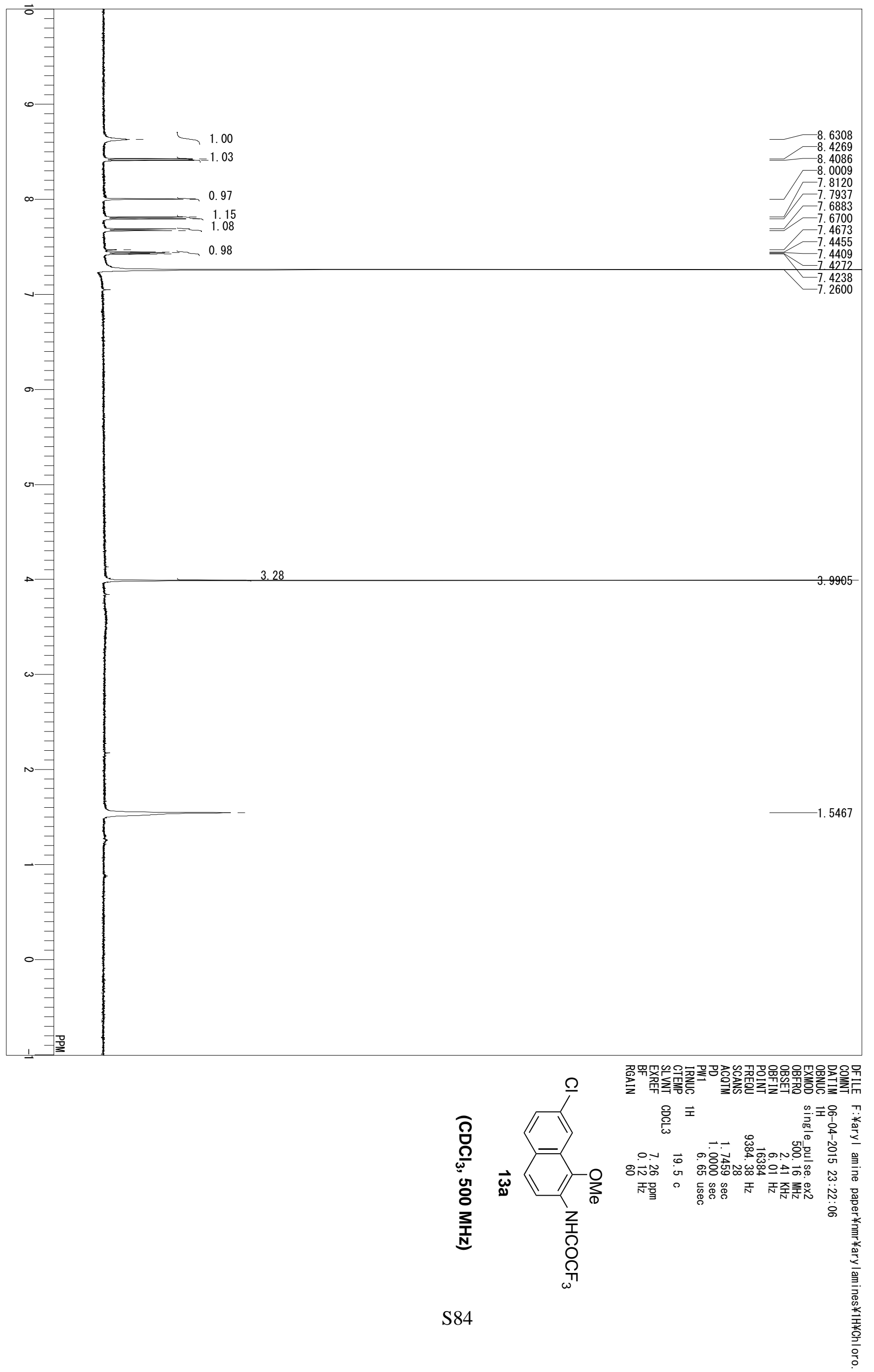


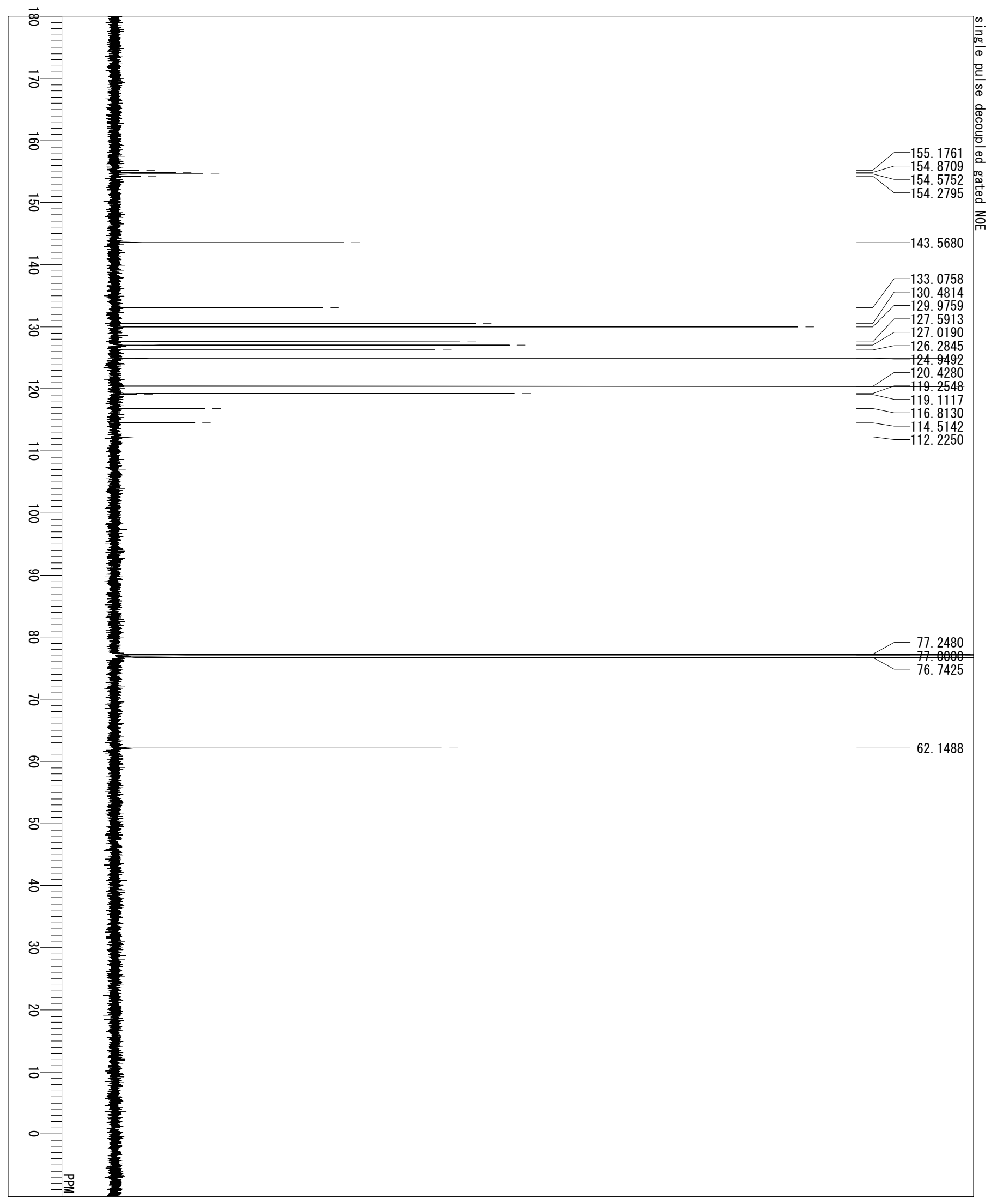

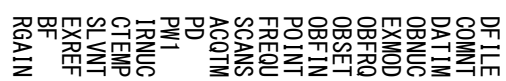

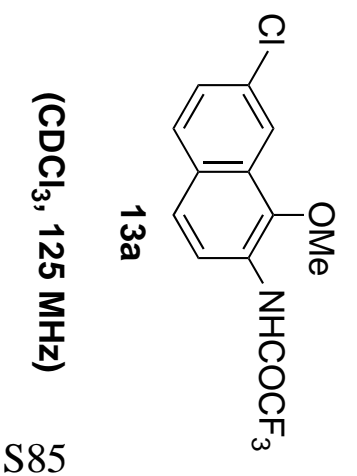

总 No 鵕 Ming

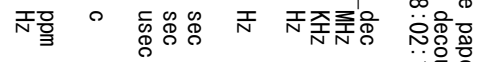




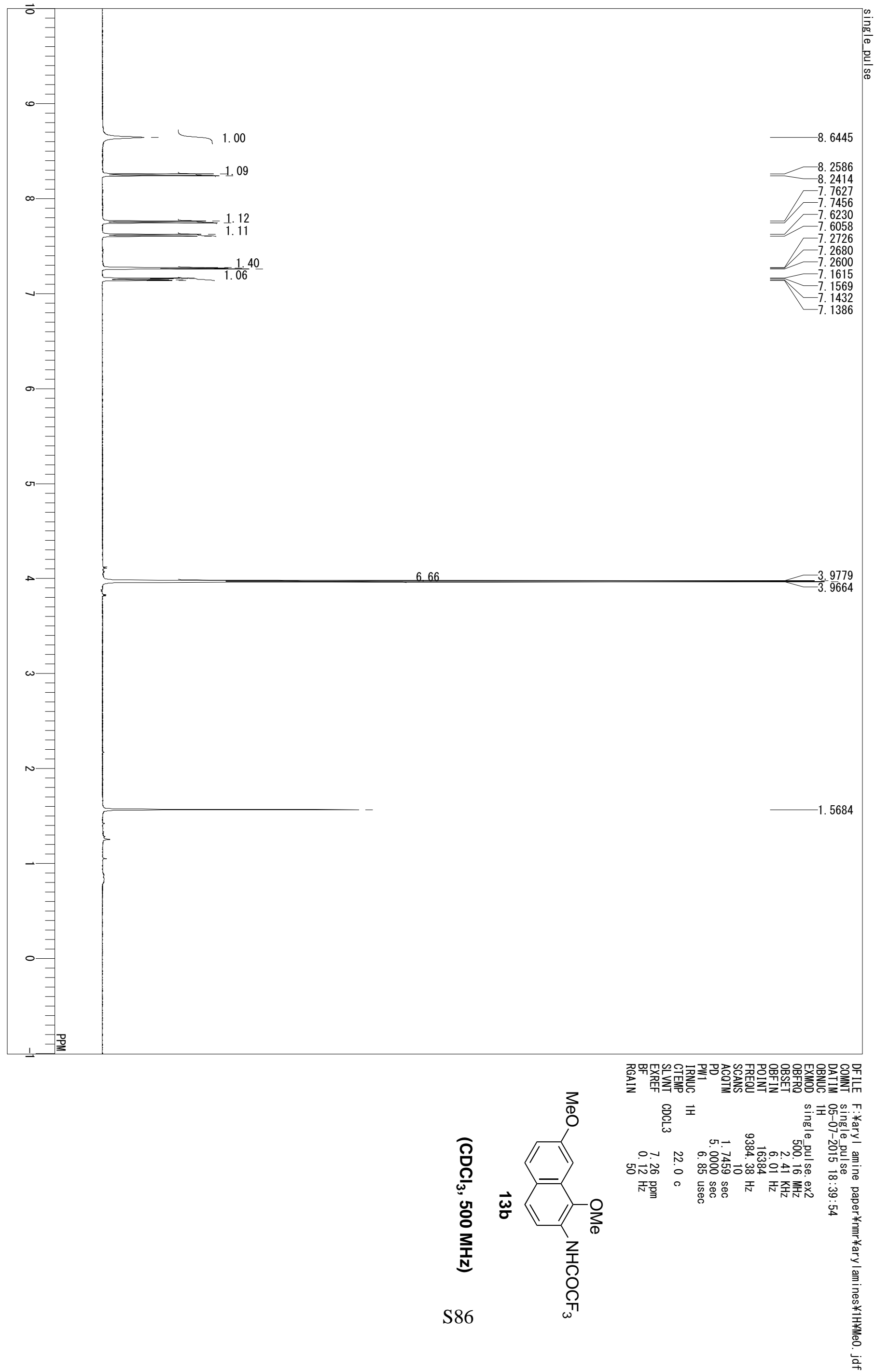



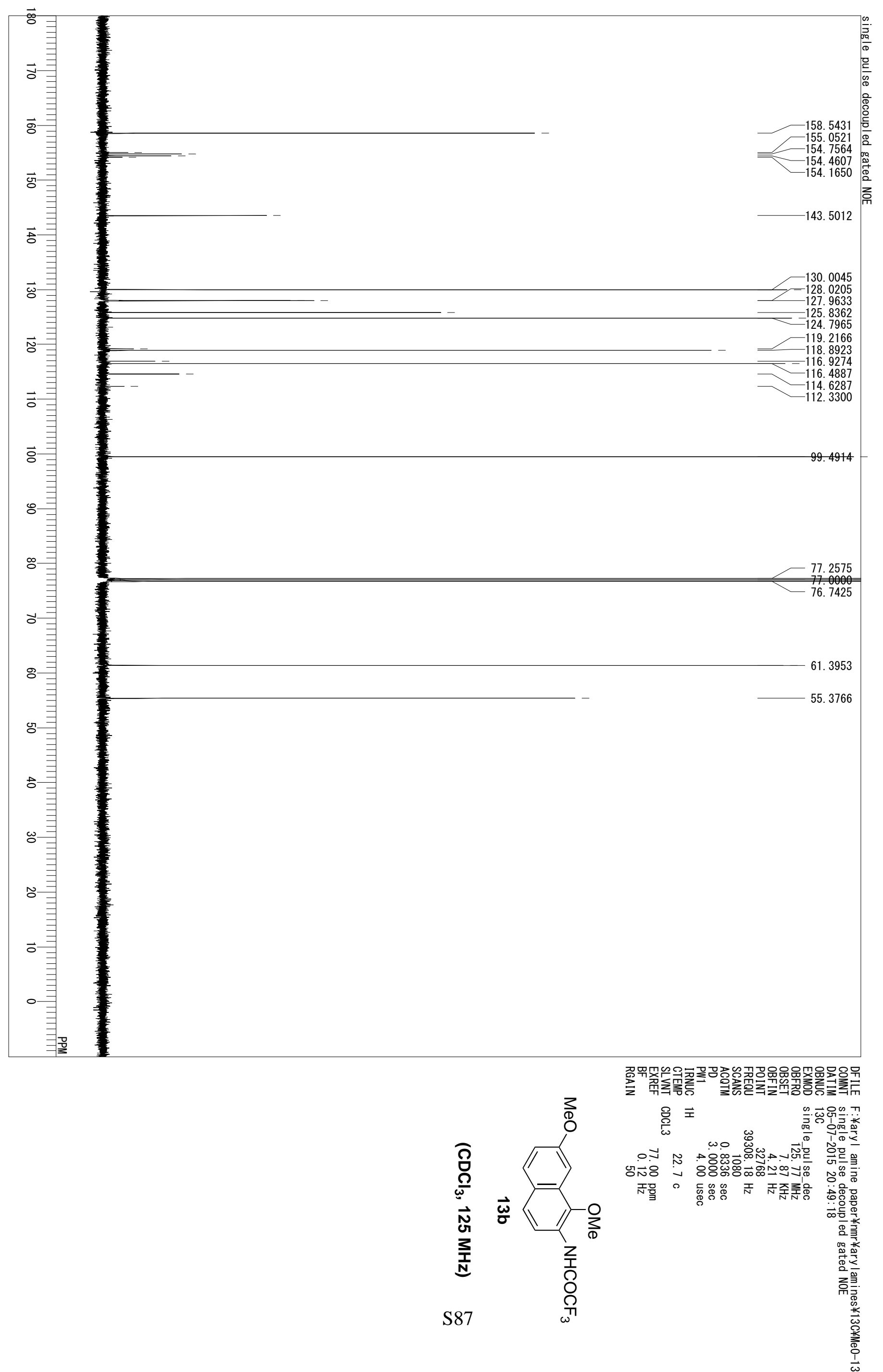


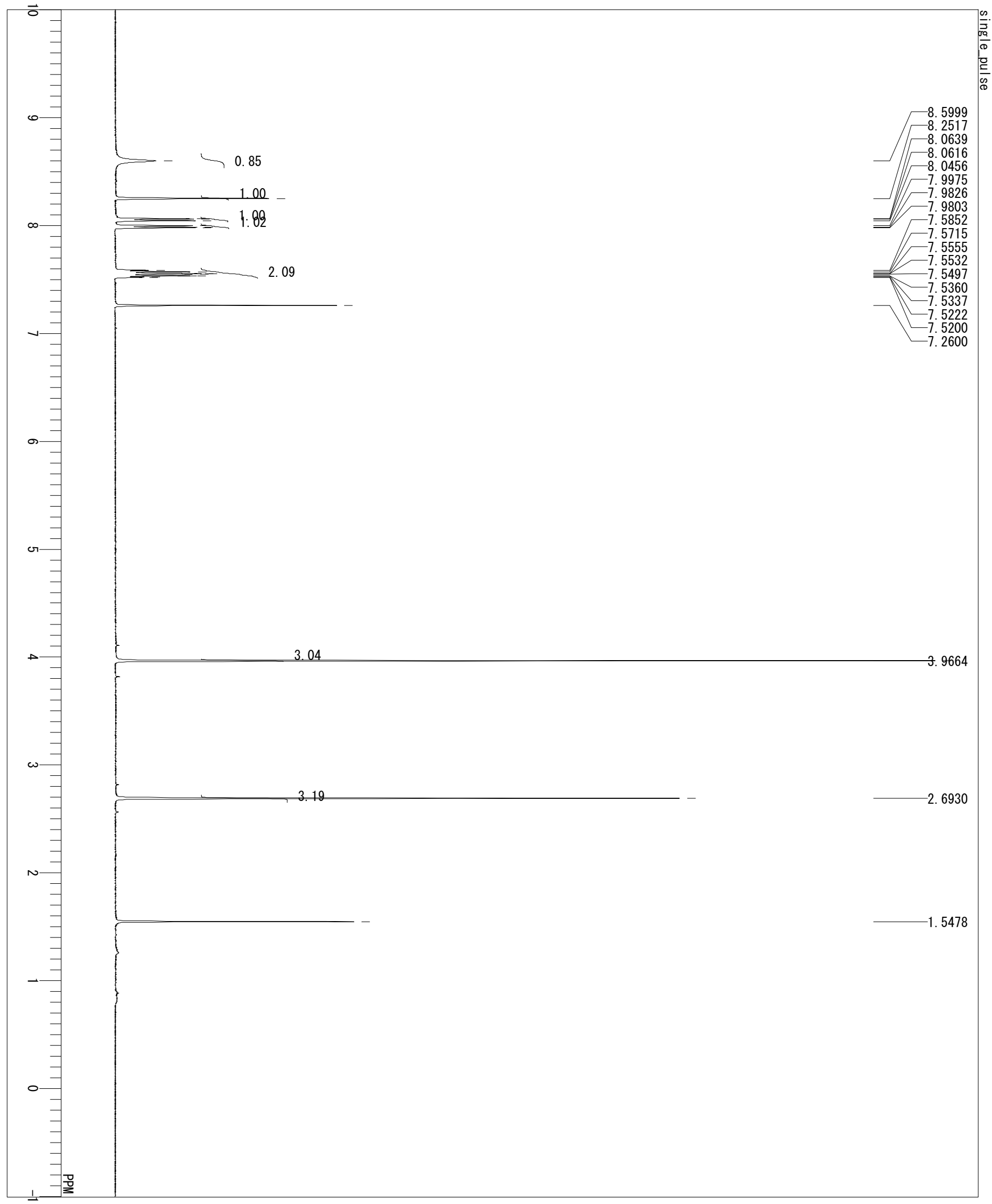

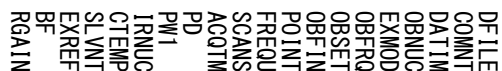

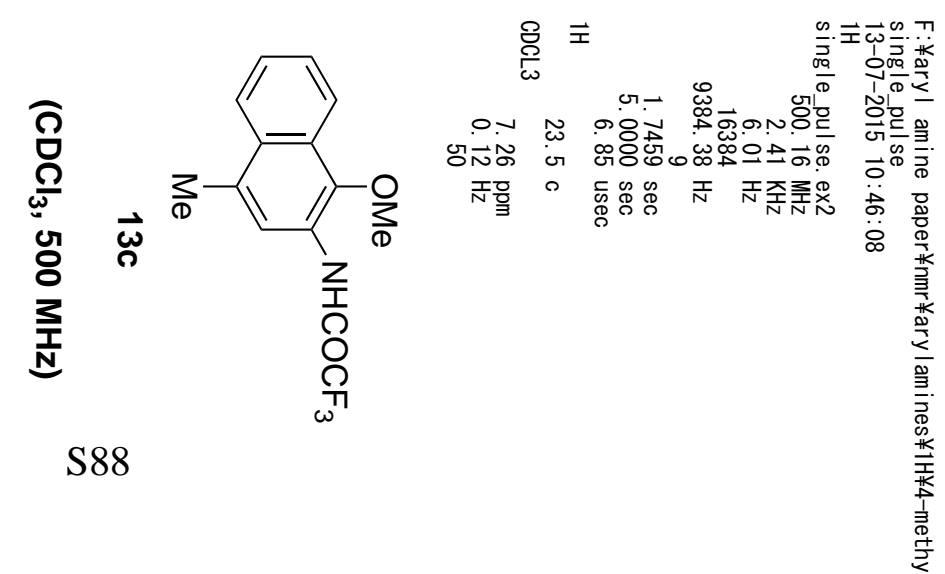



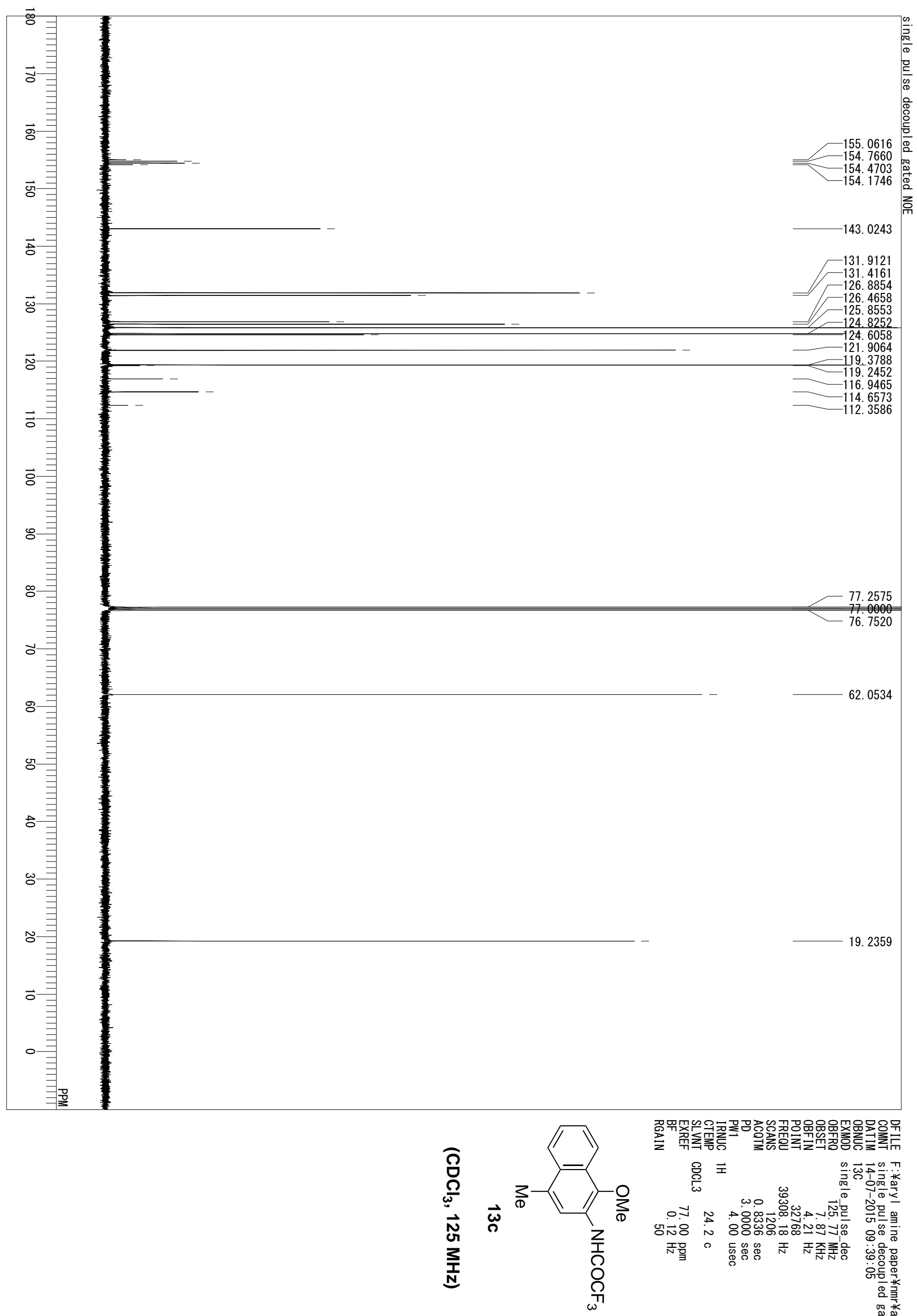

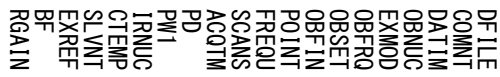

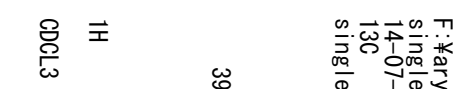

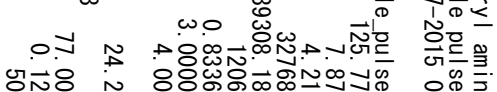

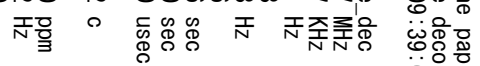




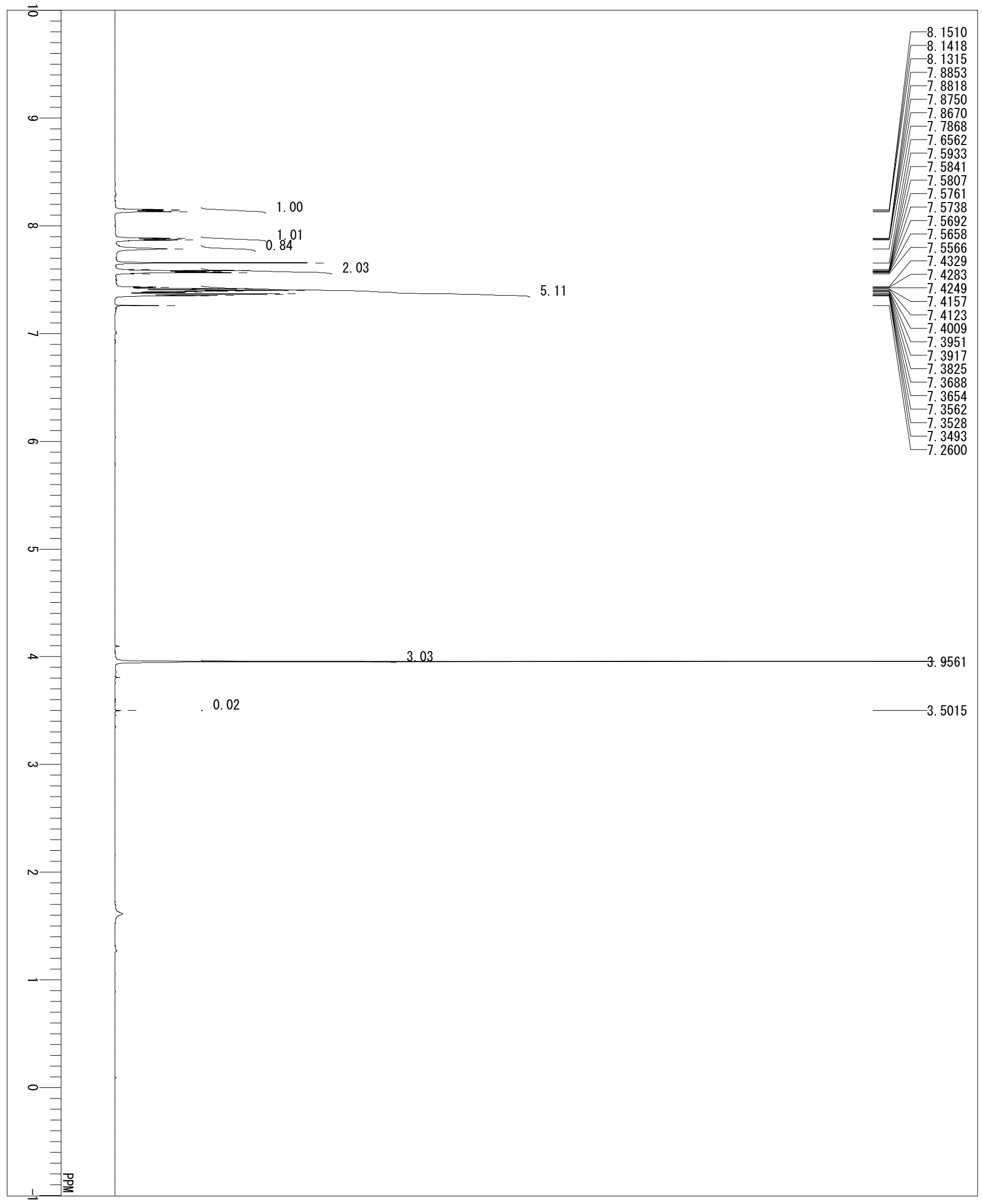

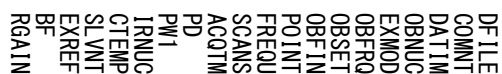

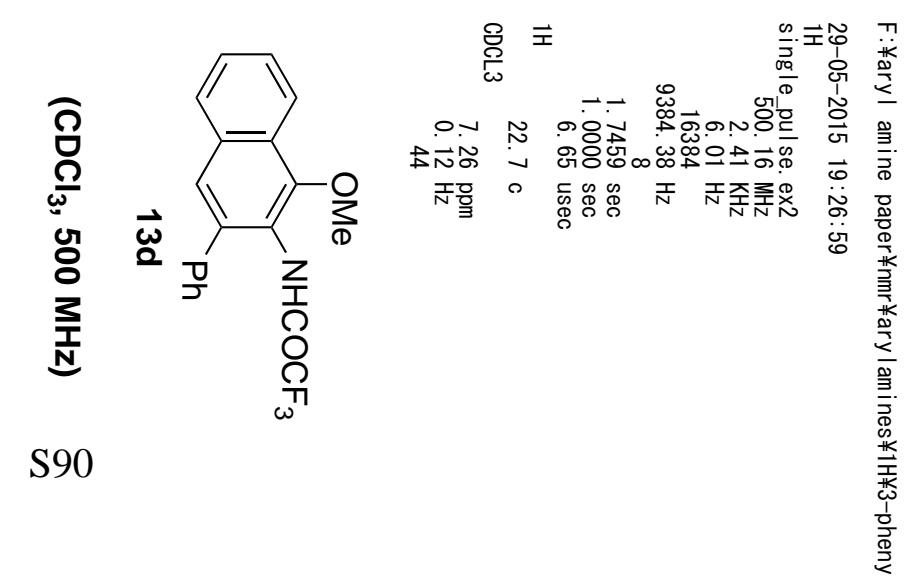




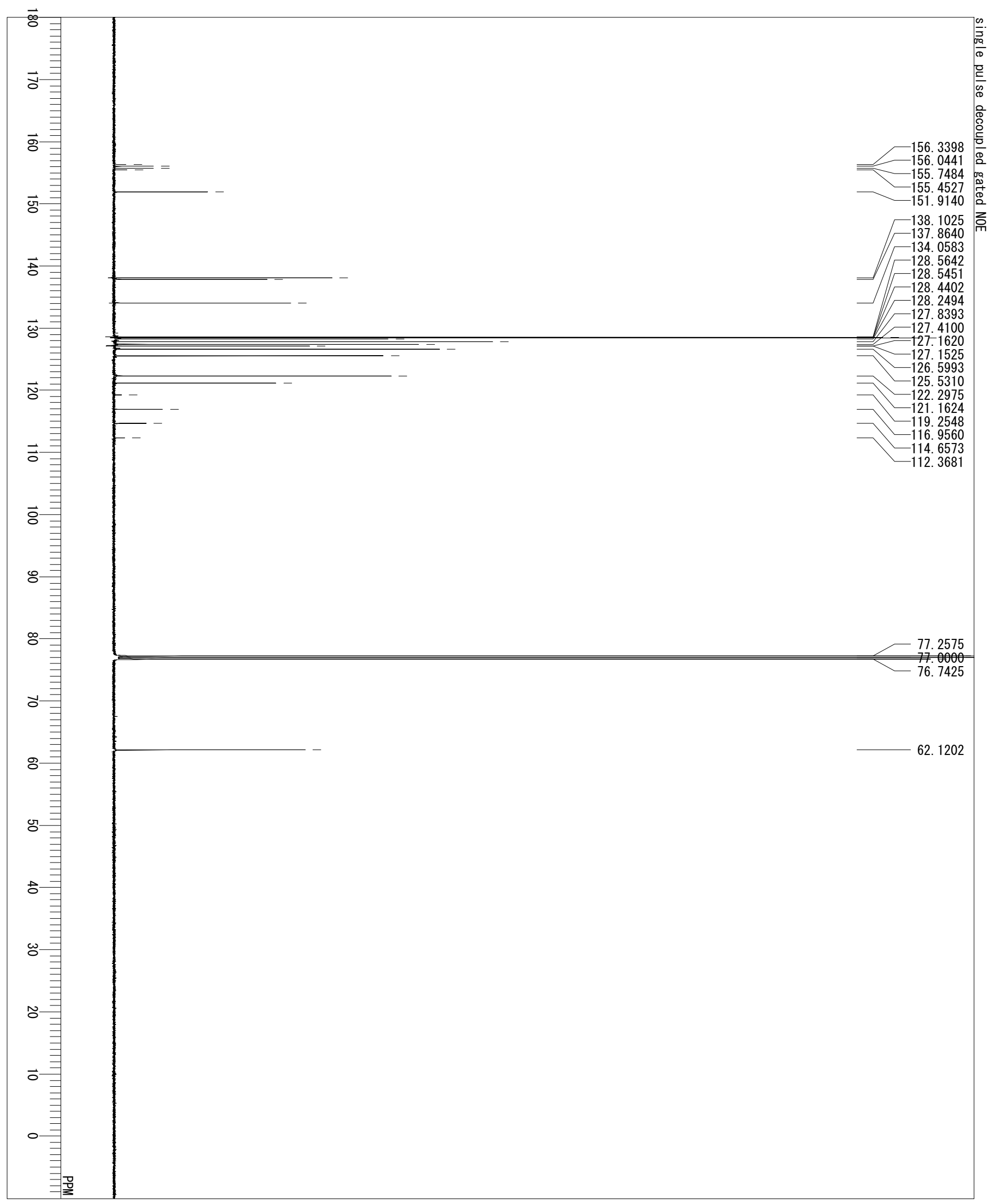

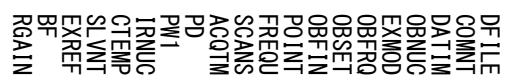

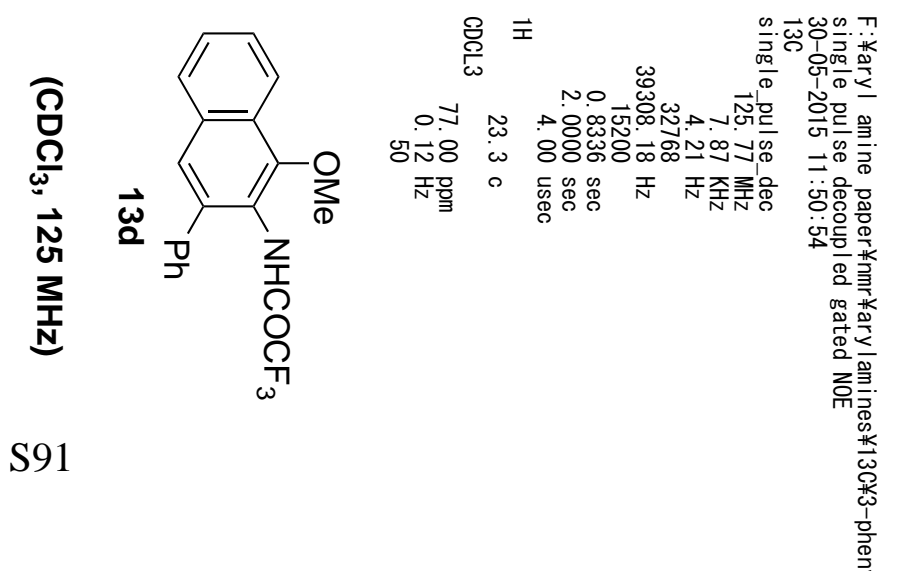




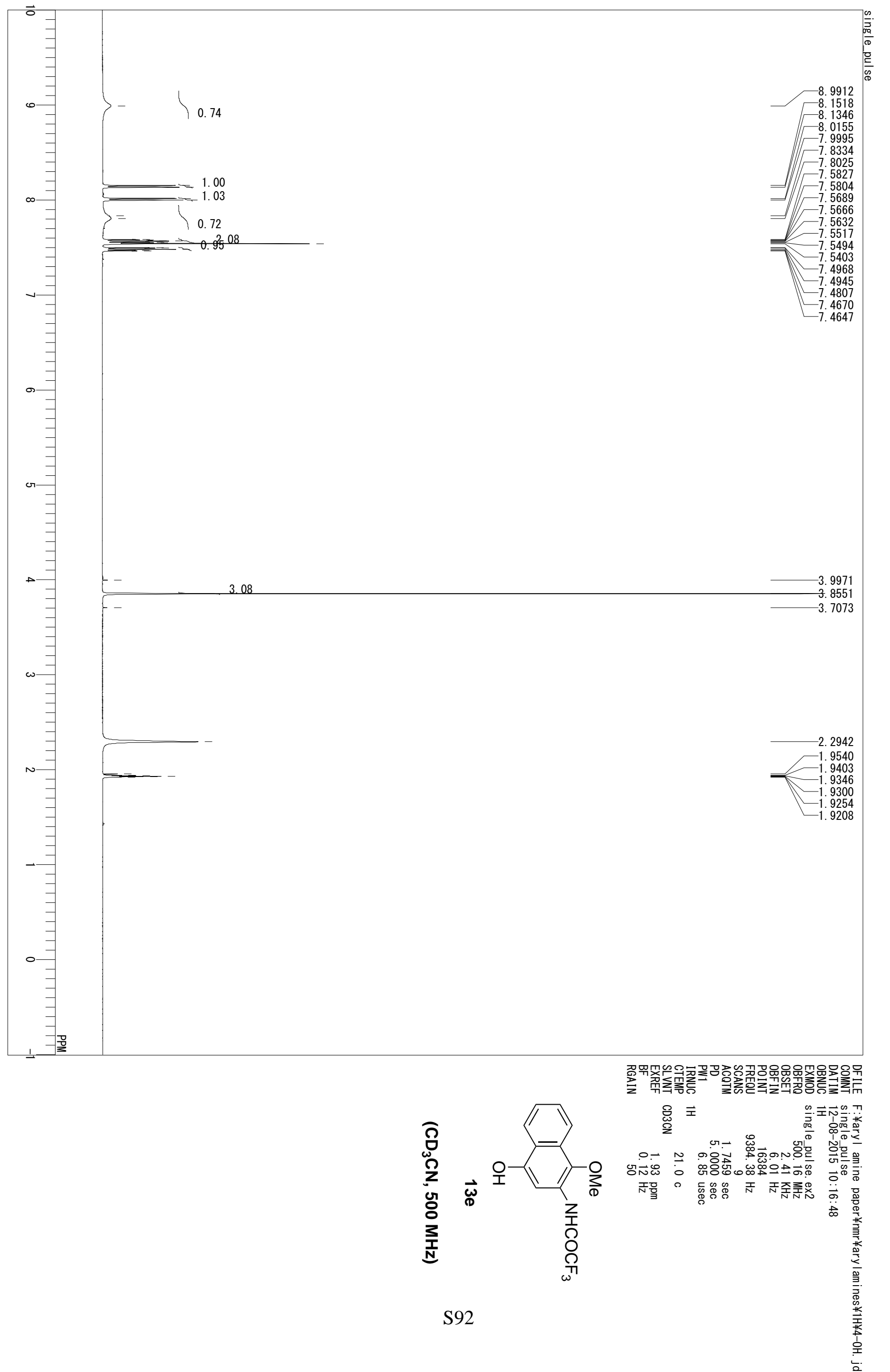




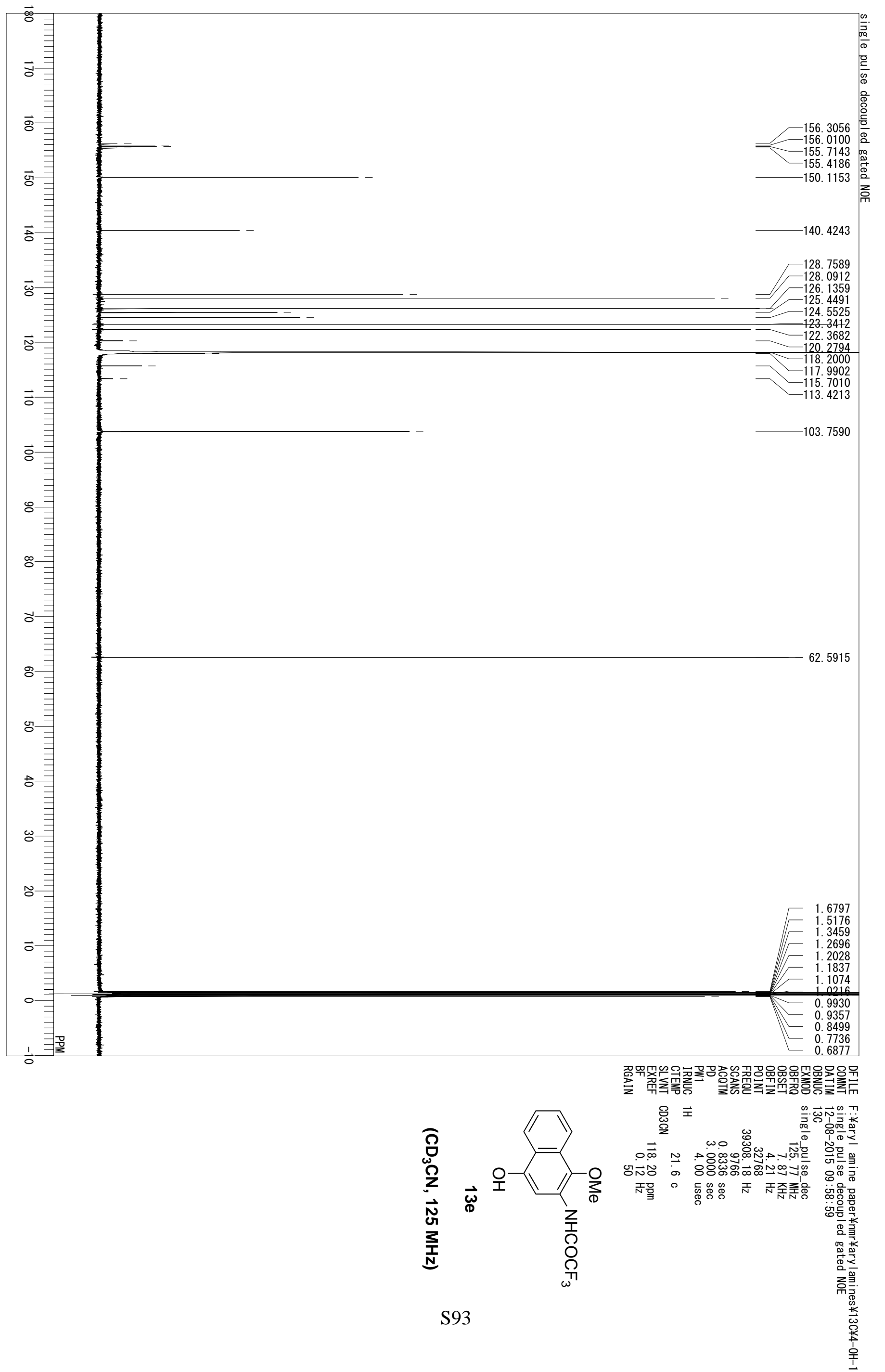




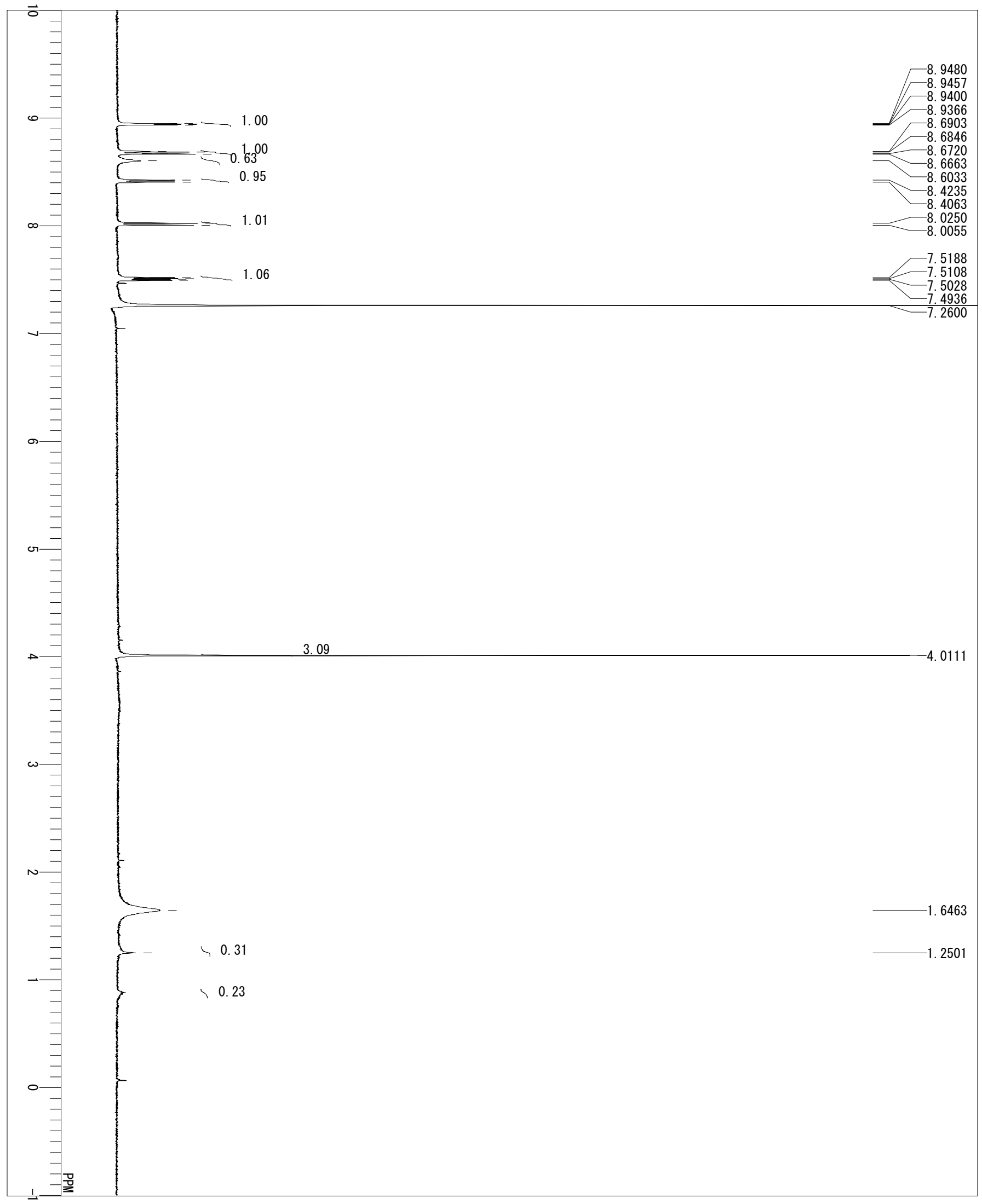

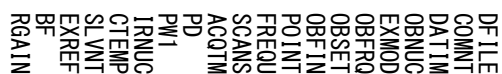

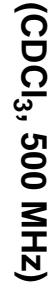
욯 $\vec{\circ}$ ․․풍

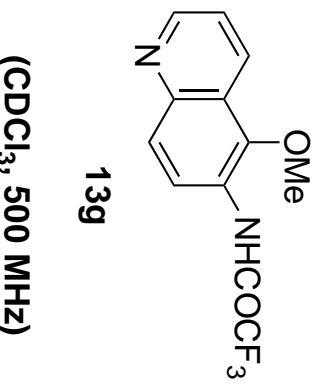

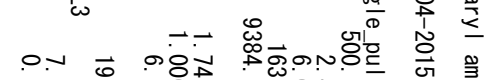
8तN

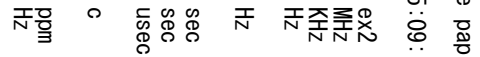
ते 


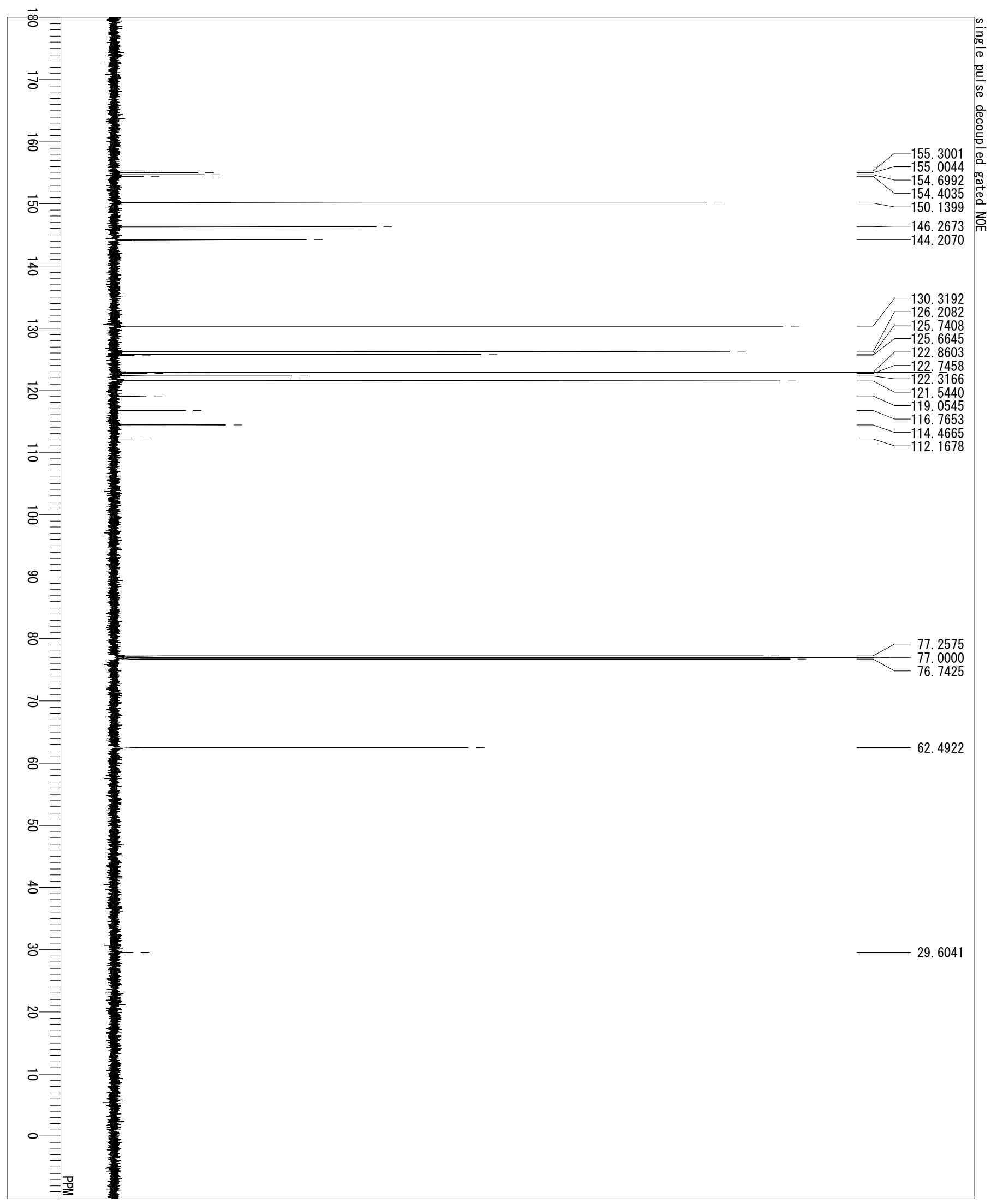

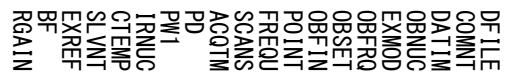

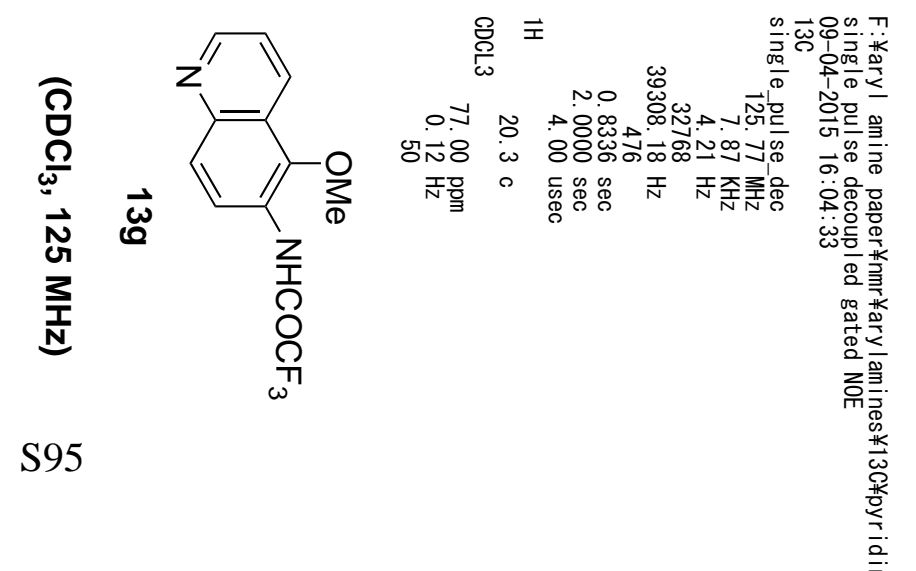




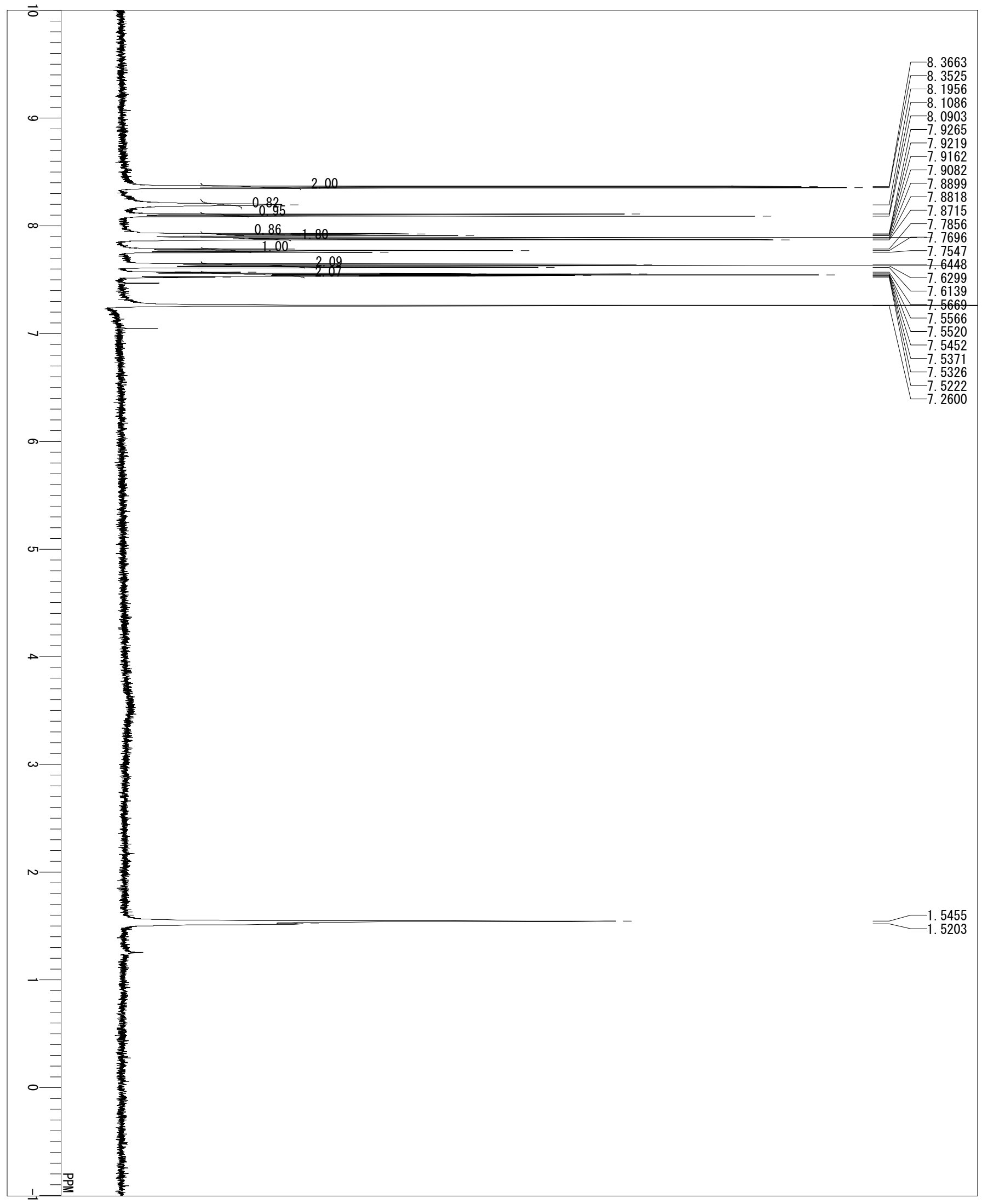

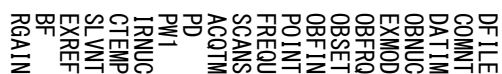

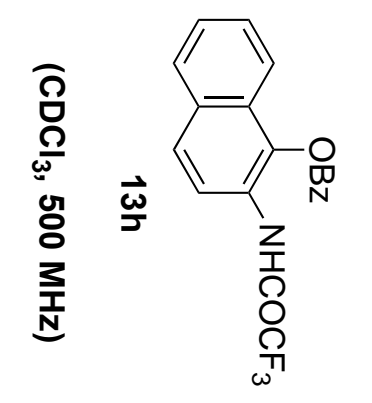

怘 $\vec{x}$

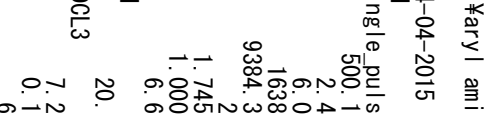

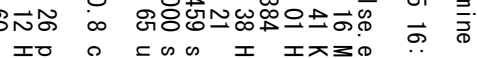

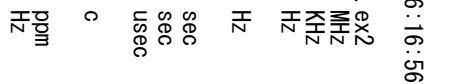




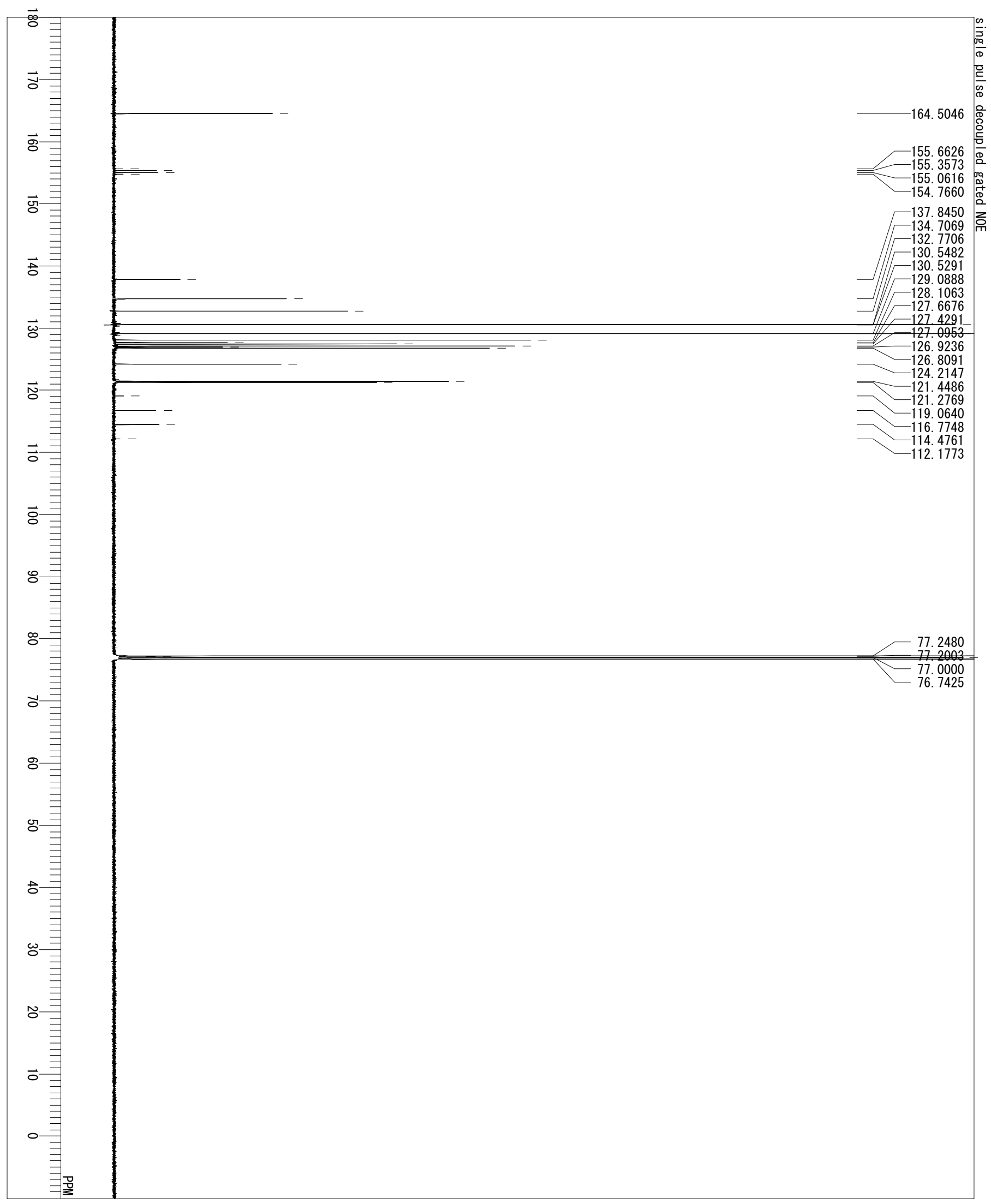

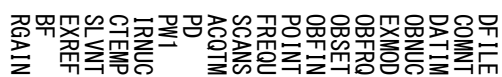

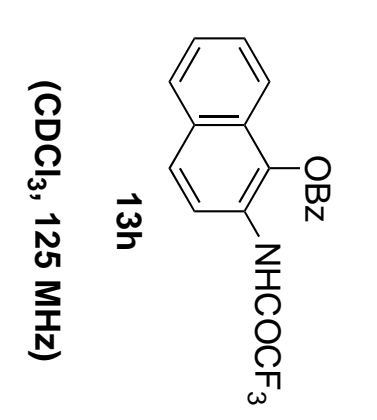

$\stackrel{8}{\stackrel{8}{\omega}} \vec{x}$

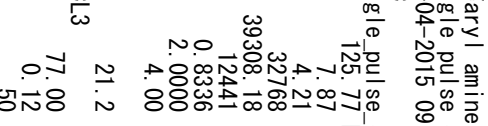

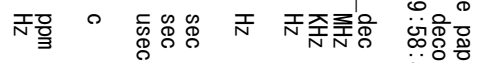

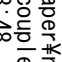




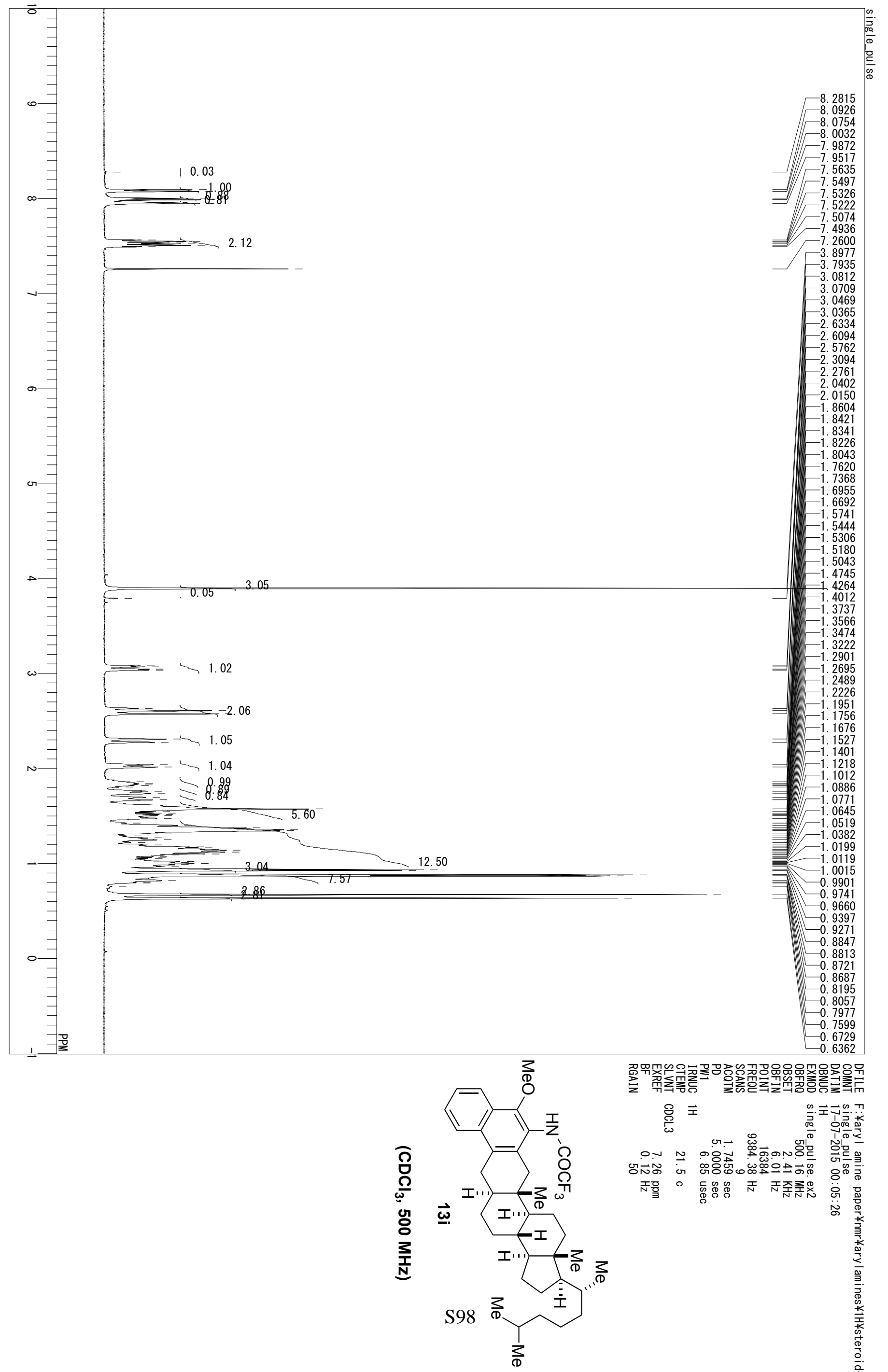



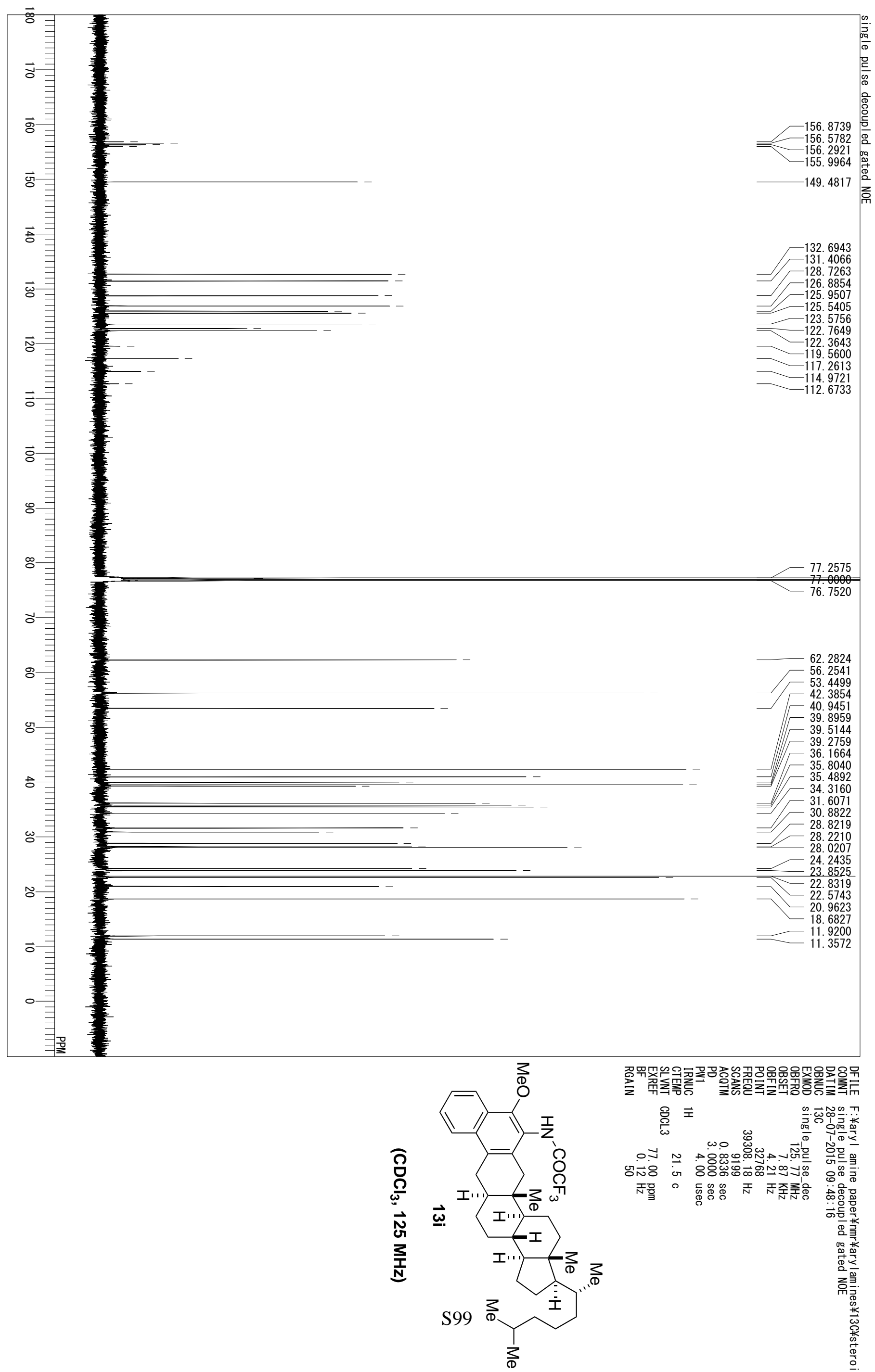


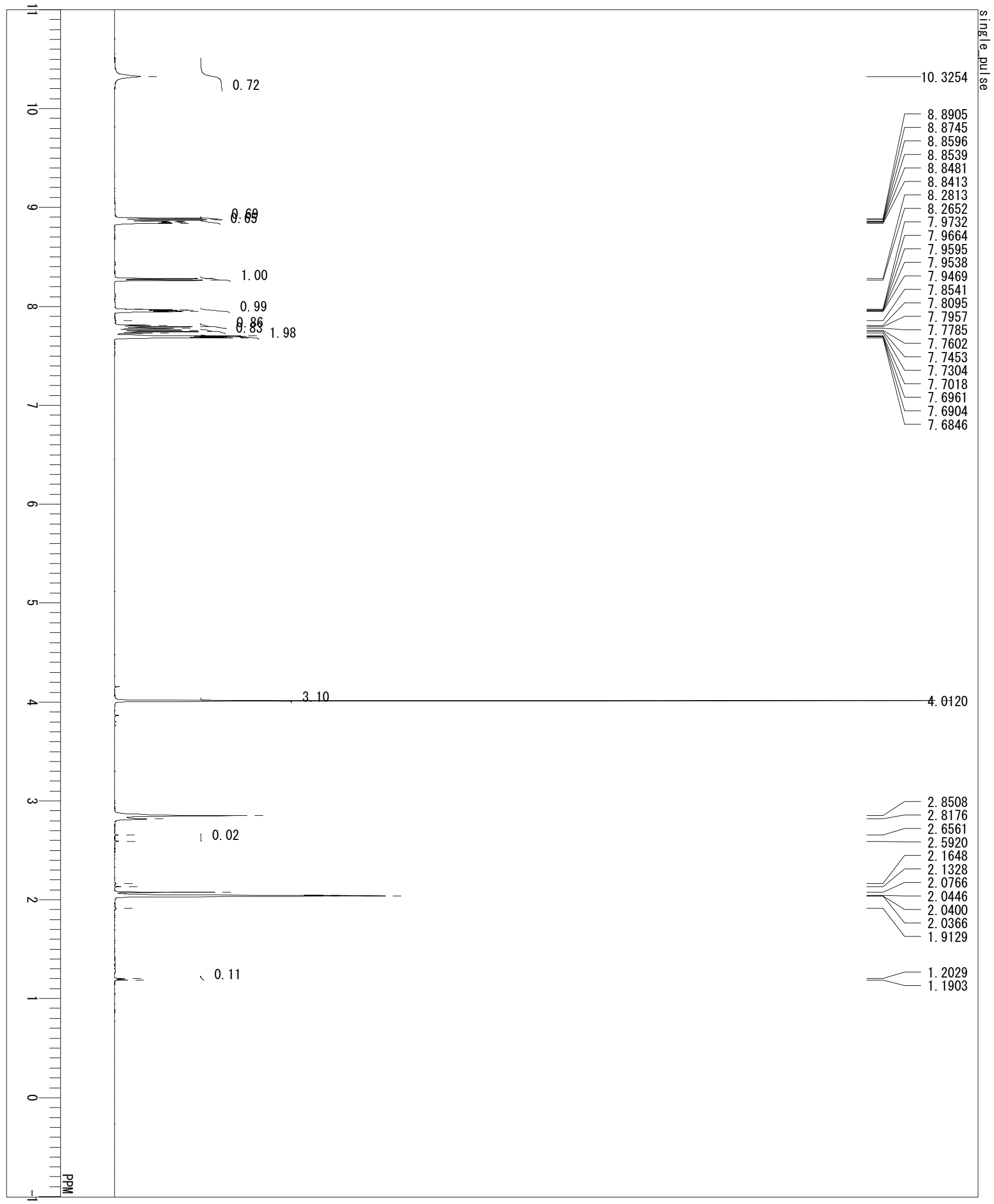

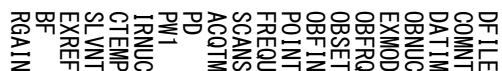

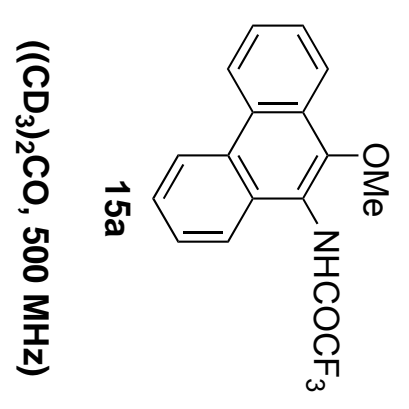

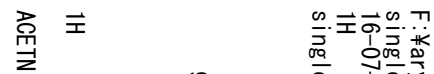
ON N $N$ N SNi

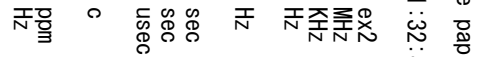
$\stackrel{\leftrightarrow}{\infty}$ 


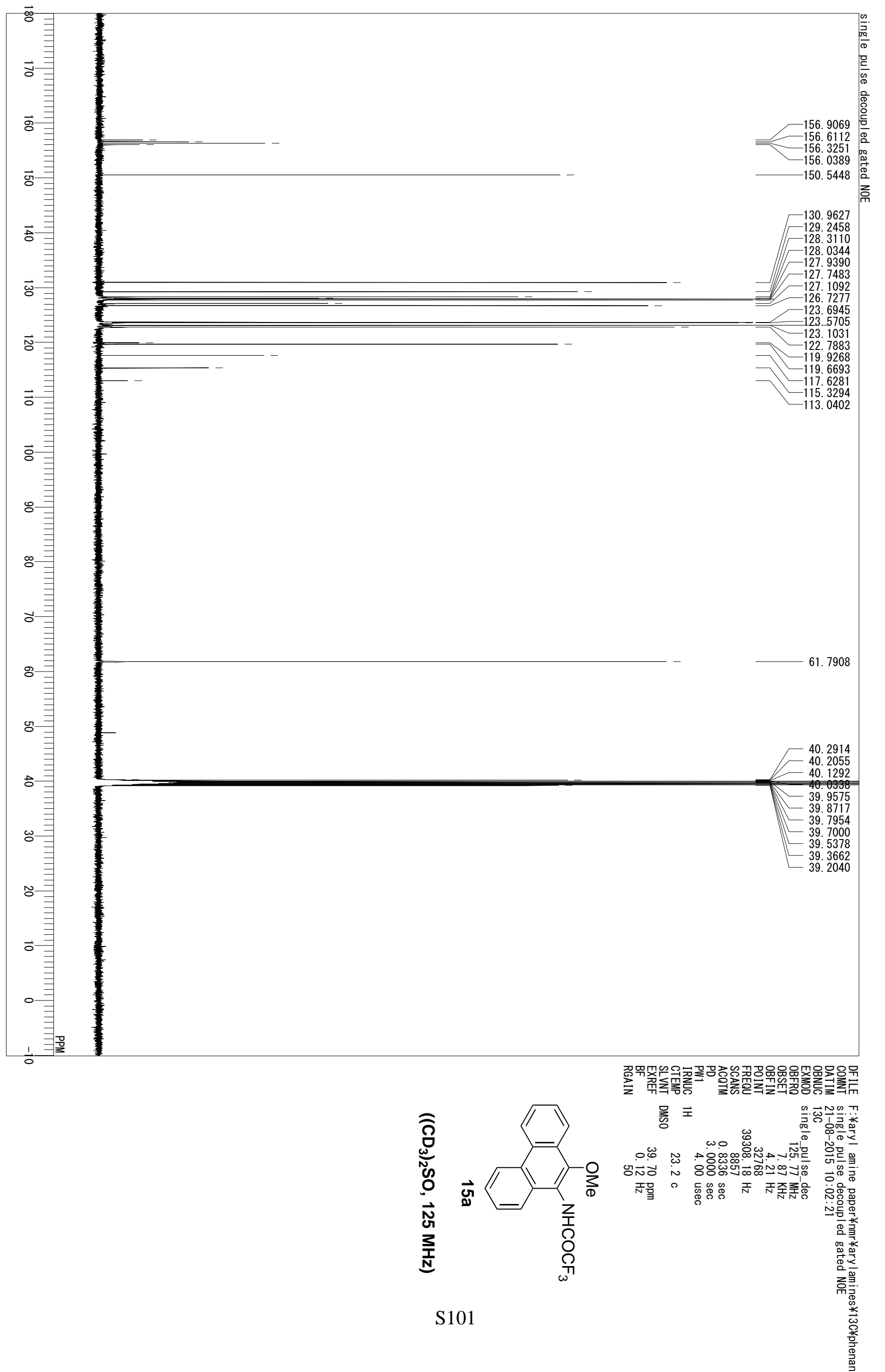




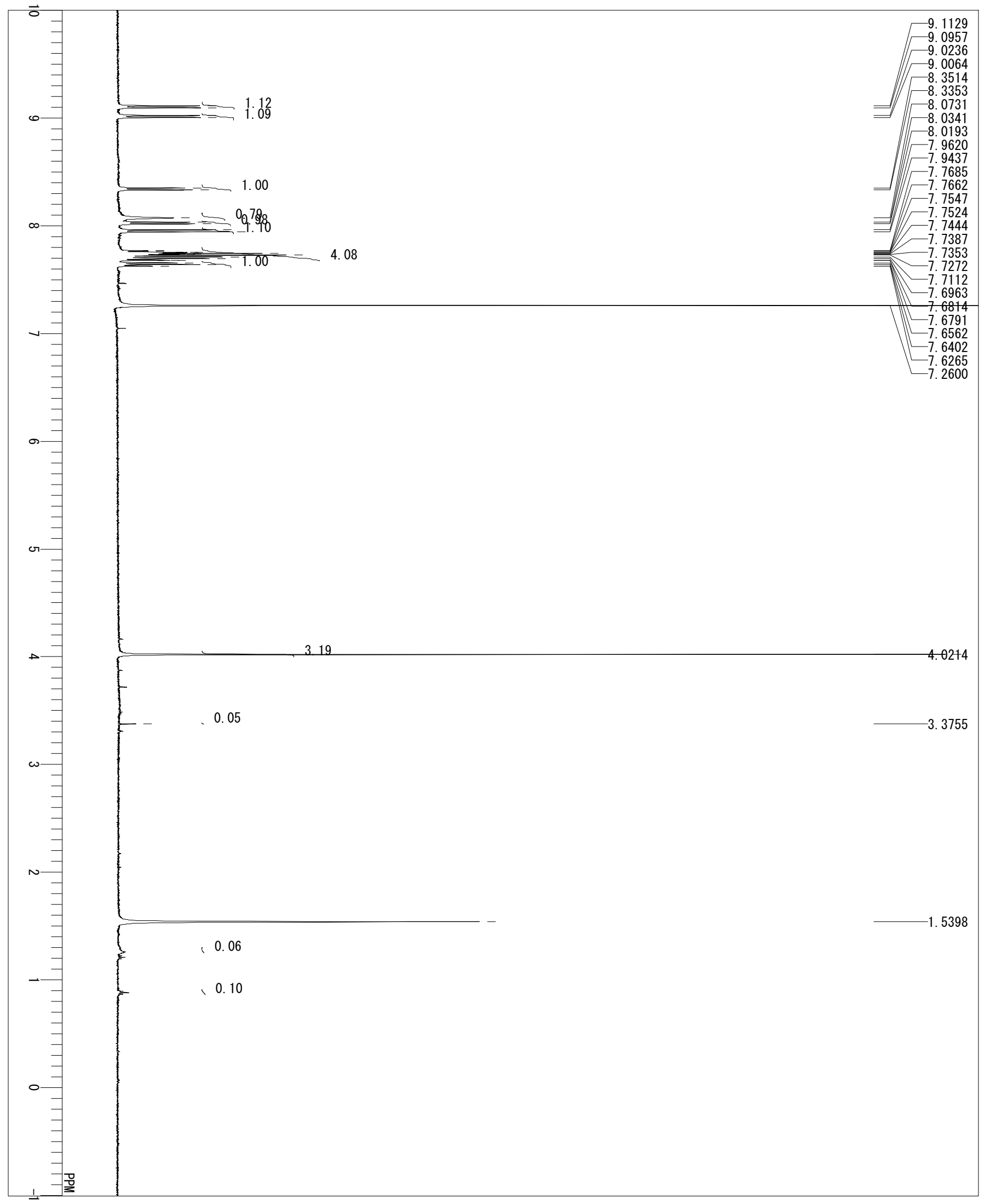

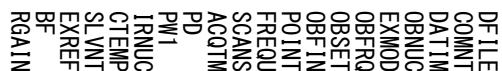

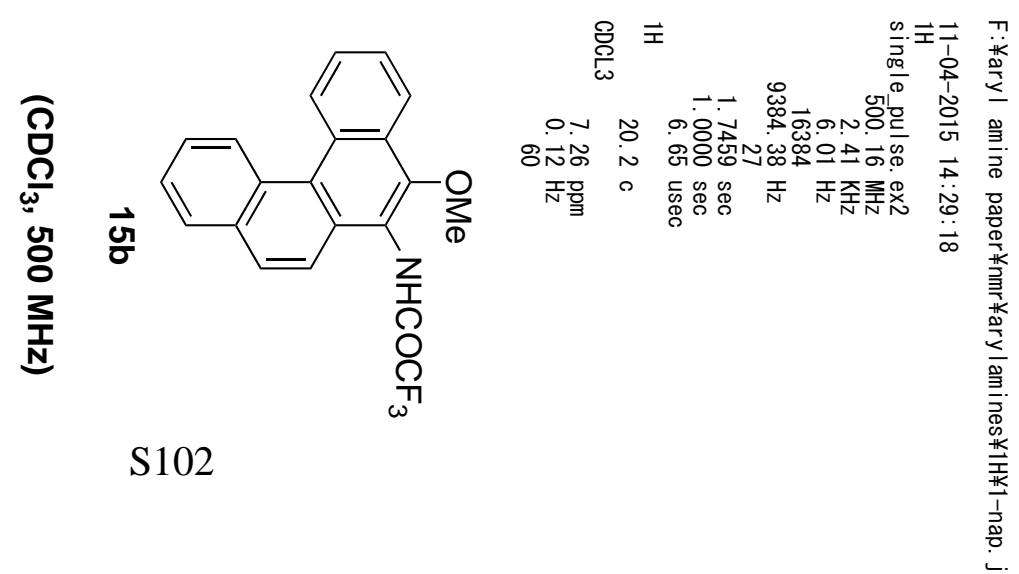




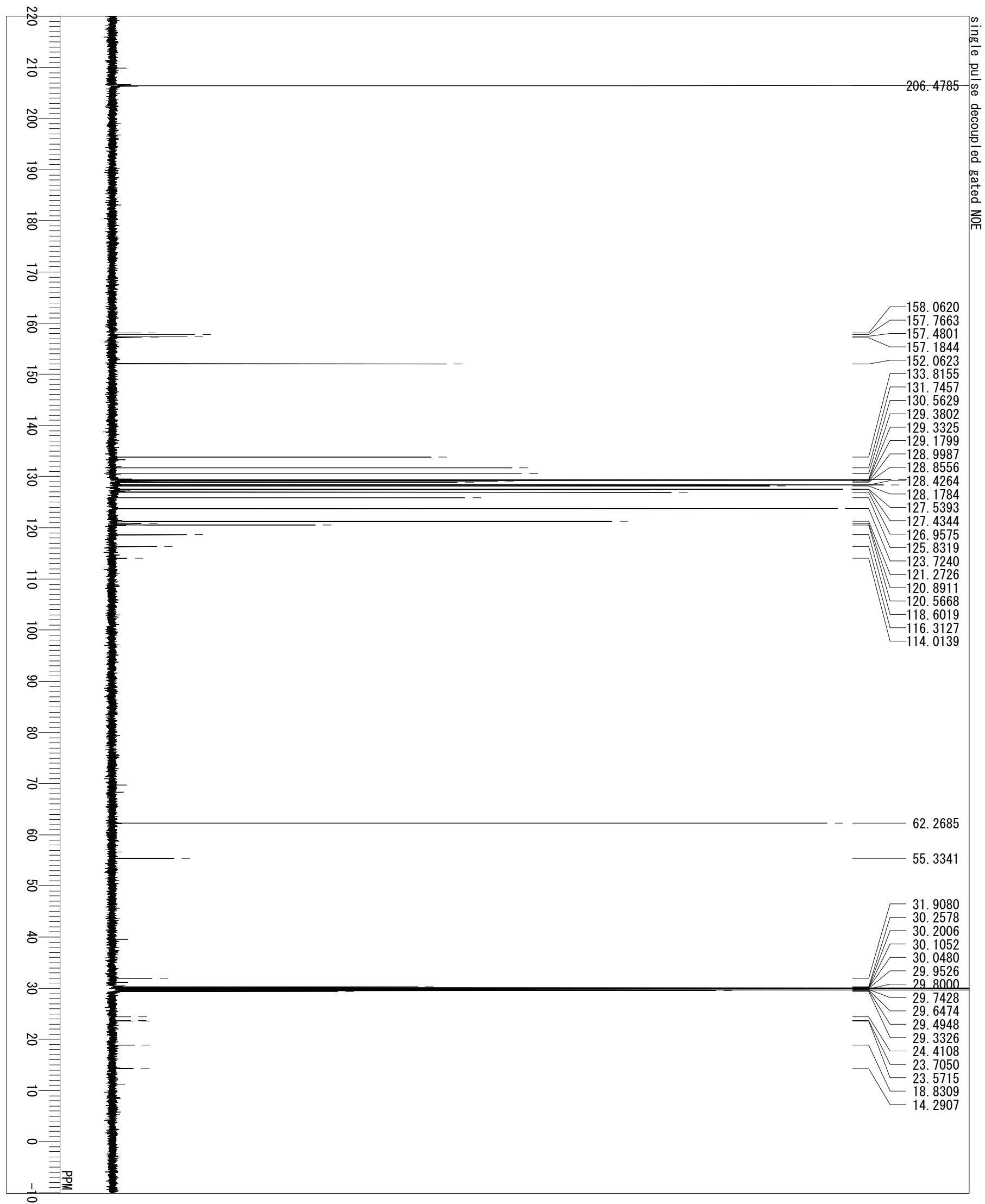

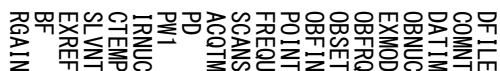

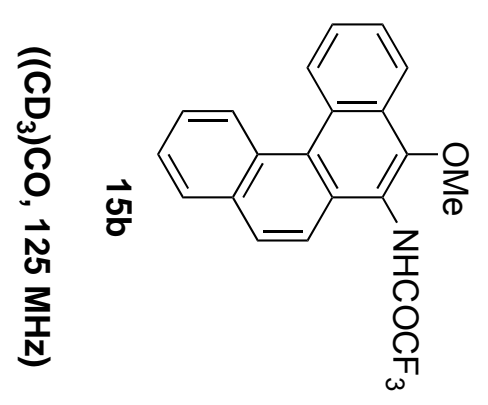

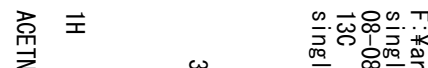

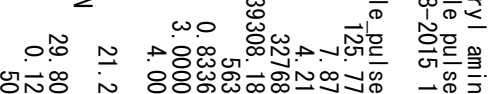

ON口。 N N

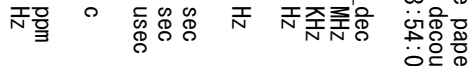




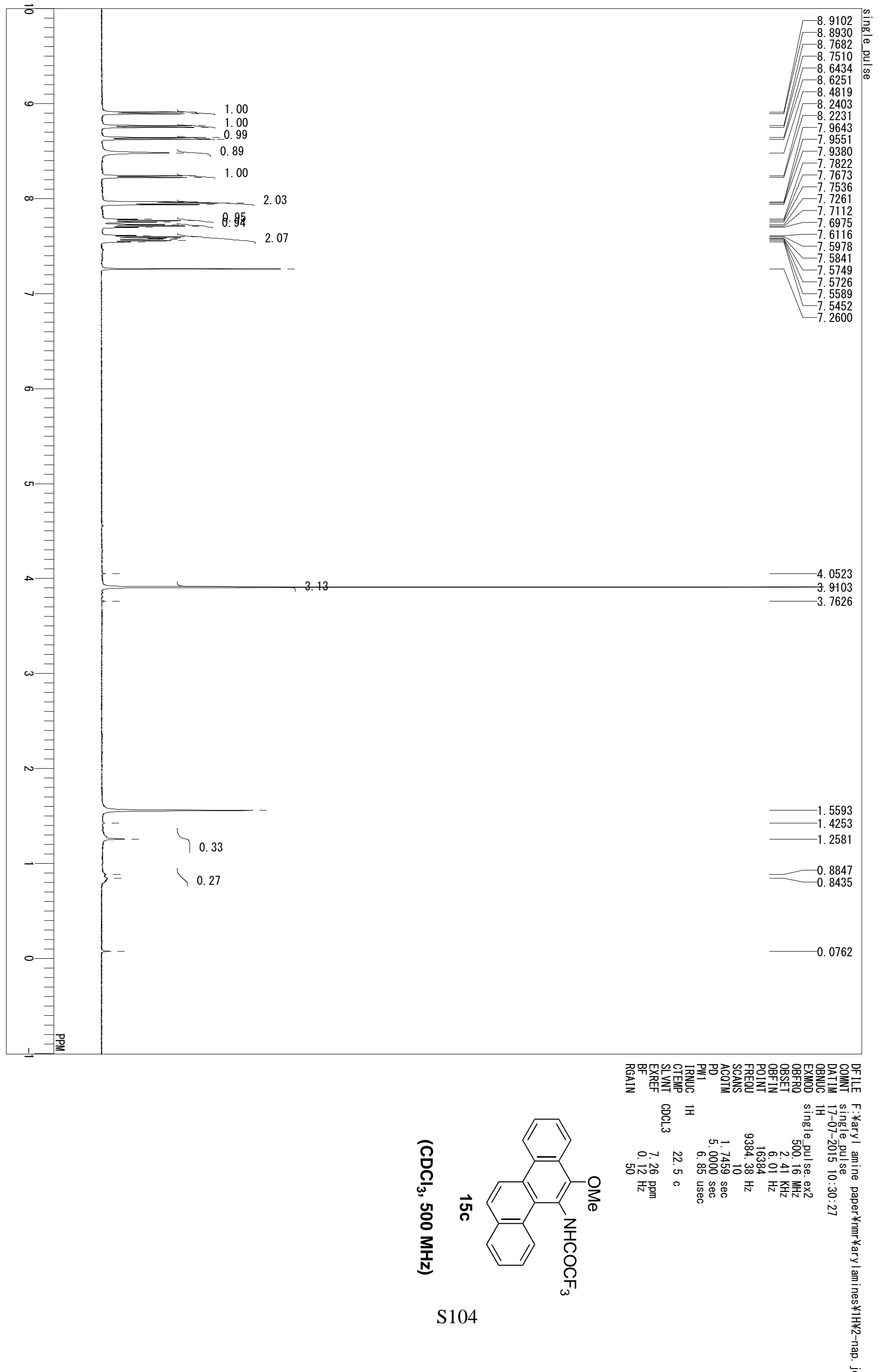




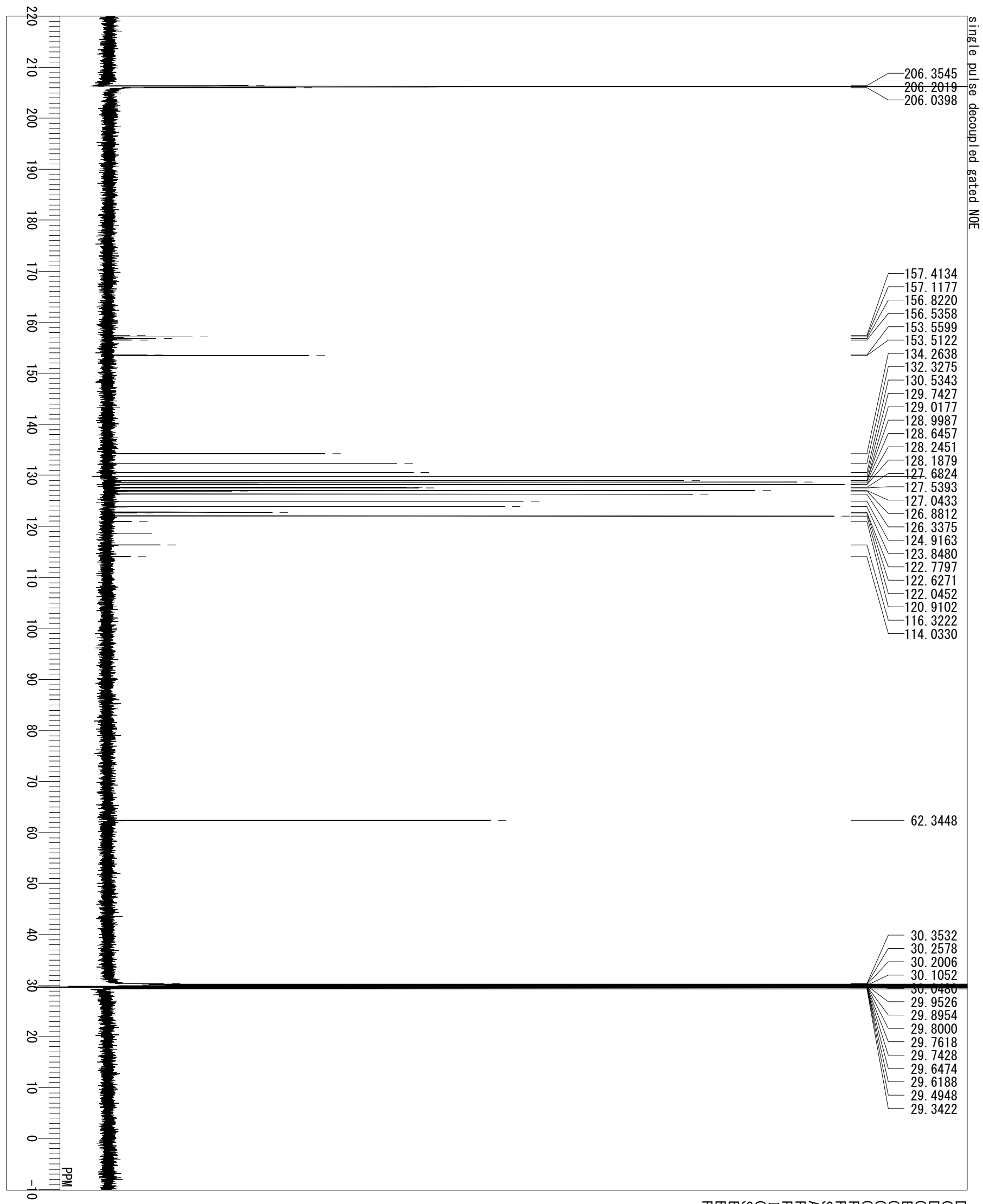

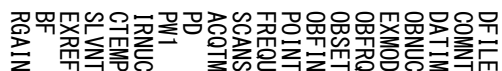

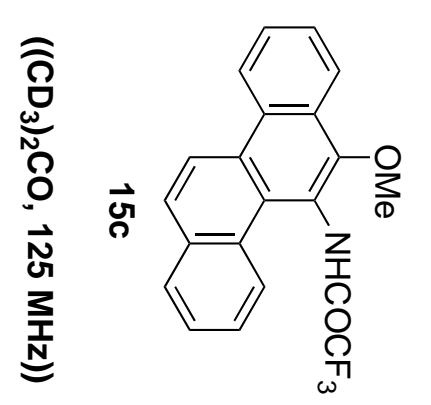

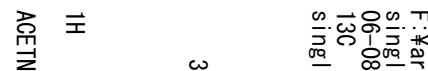

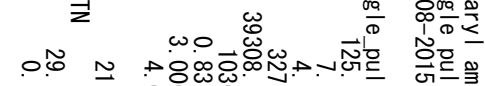

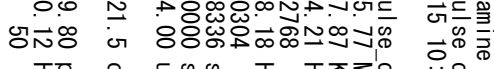

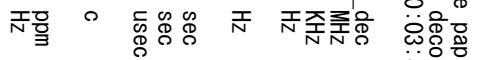


:

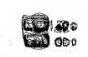

$\because$

?:

$\infty$

\section{(1)}

$\stackrel{\infty}{+}$

$\infty$

$\underset{i}{\infty}$

\%

ร9ำ

$\infty$

$\infty$

$\because$

\%

3

ำ

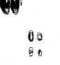

$\because$

$\stackrel{\infty}{\infty}$

ज.

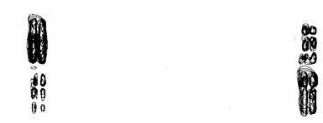

路

9

ำ

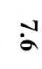

盟

露

暍

is

$\because$

i

in

$\div$

$\because$

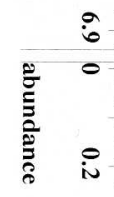

$+$

$: \frac{9}{9}$

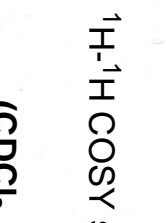

잉 


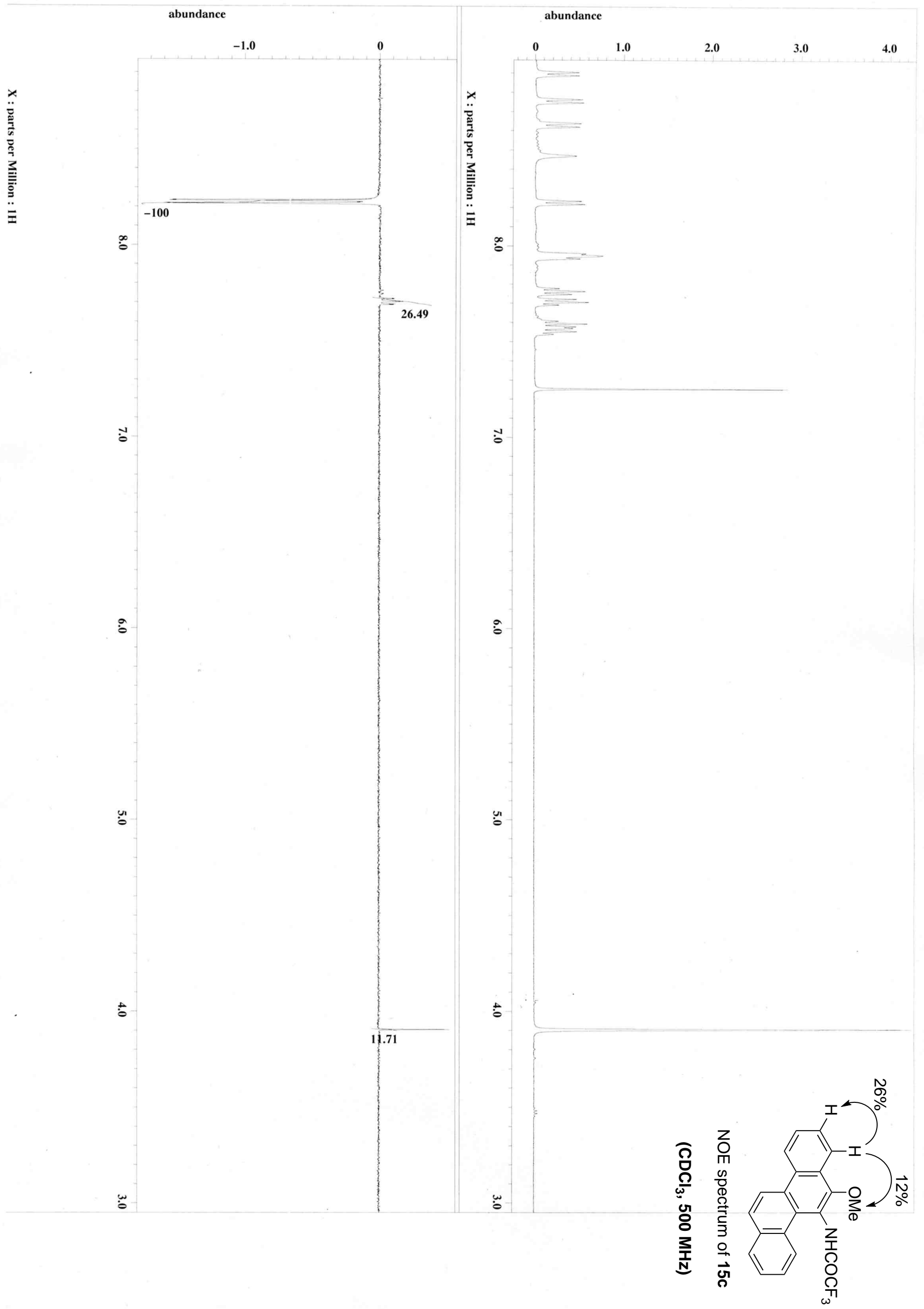

S107 


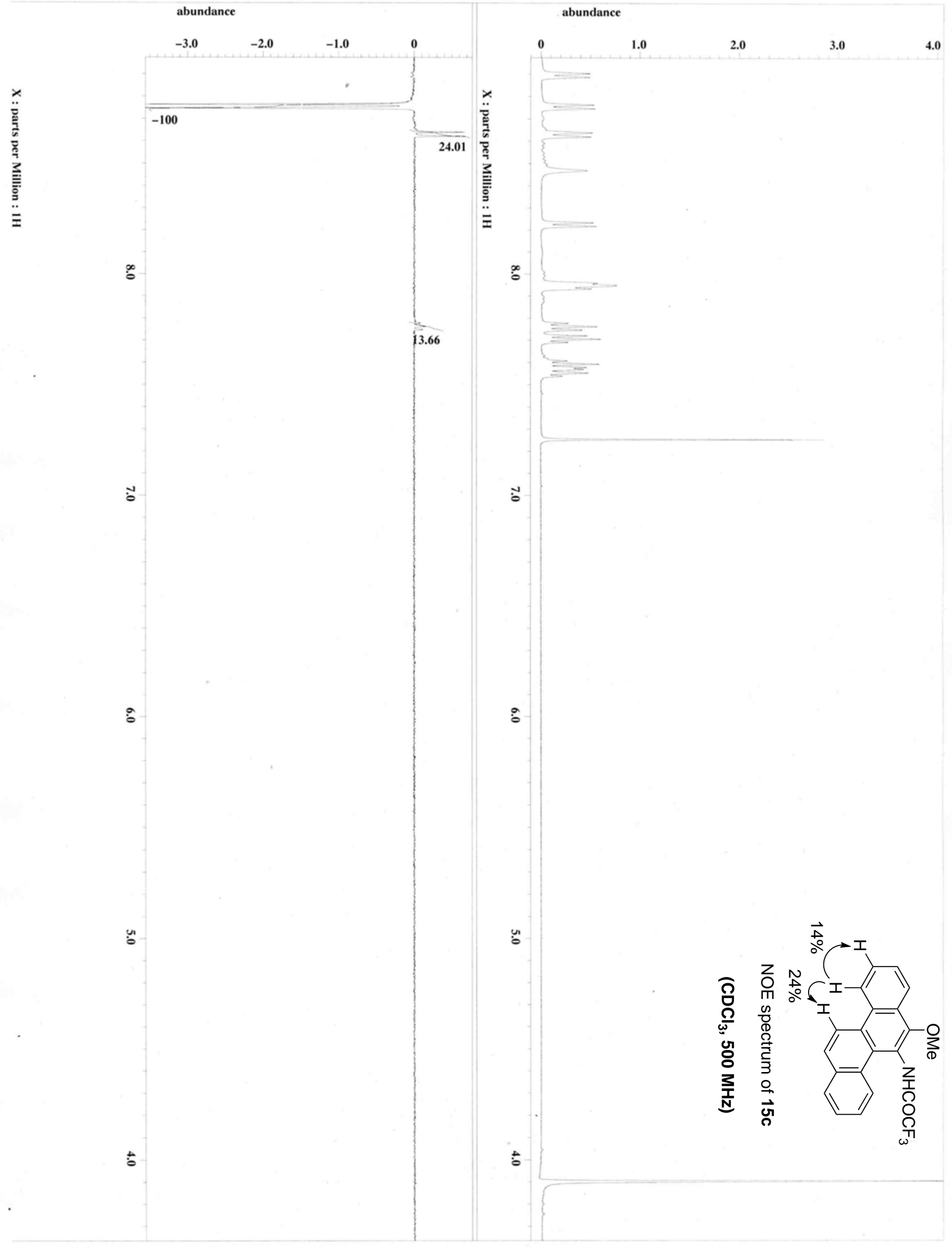




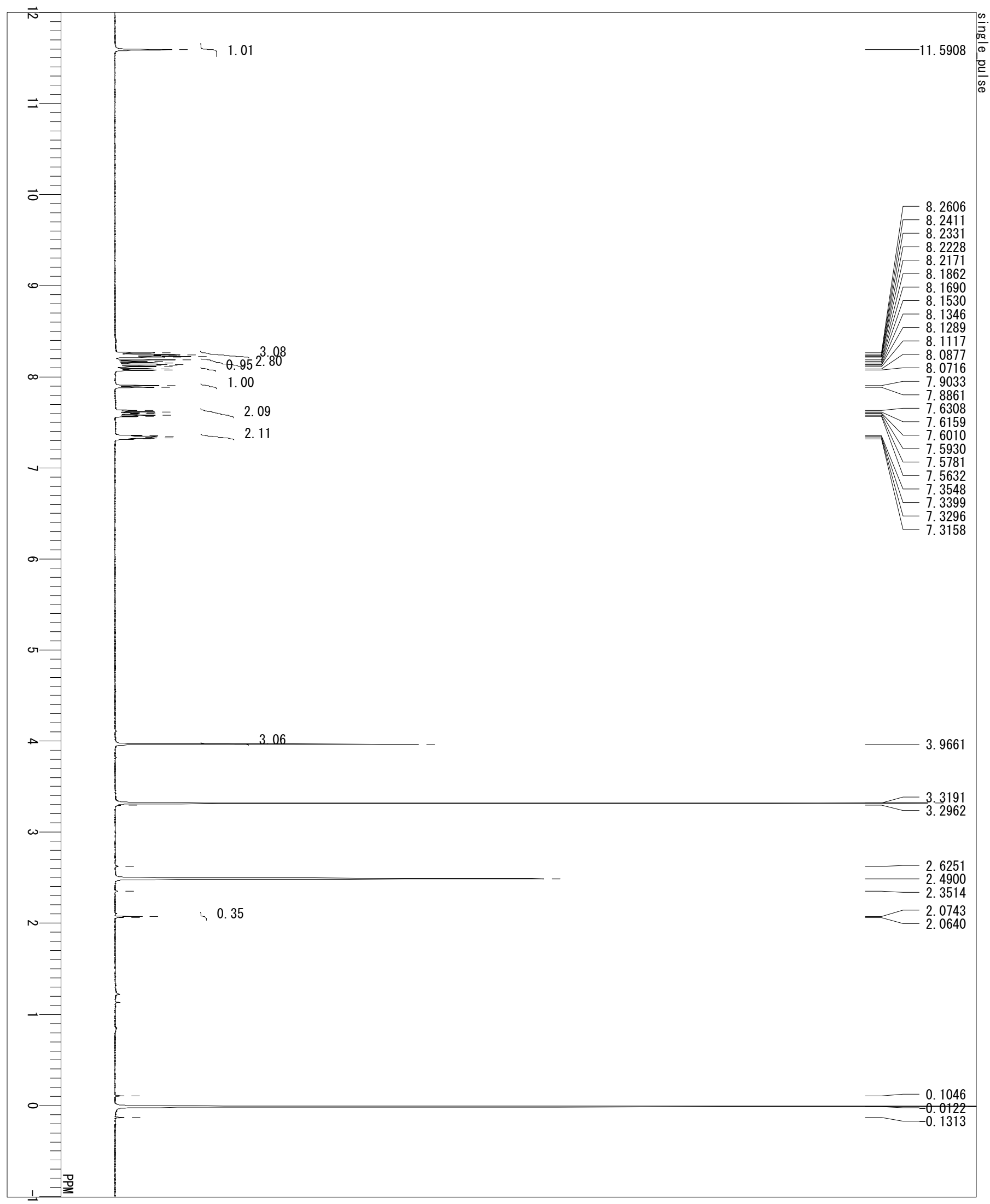

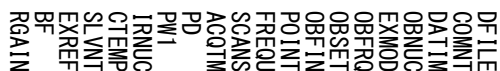

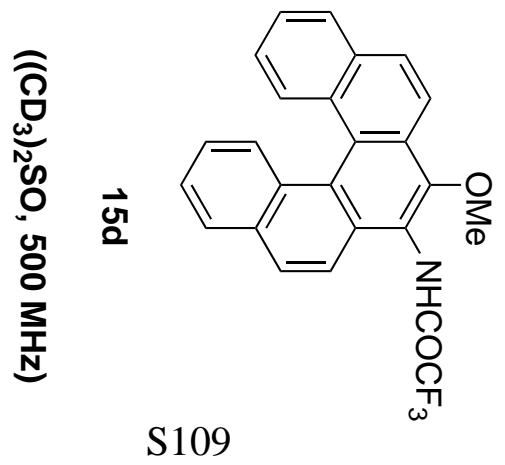

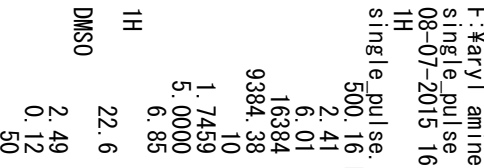

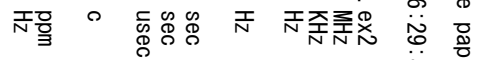




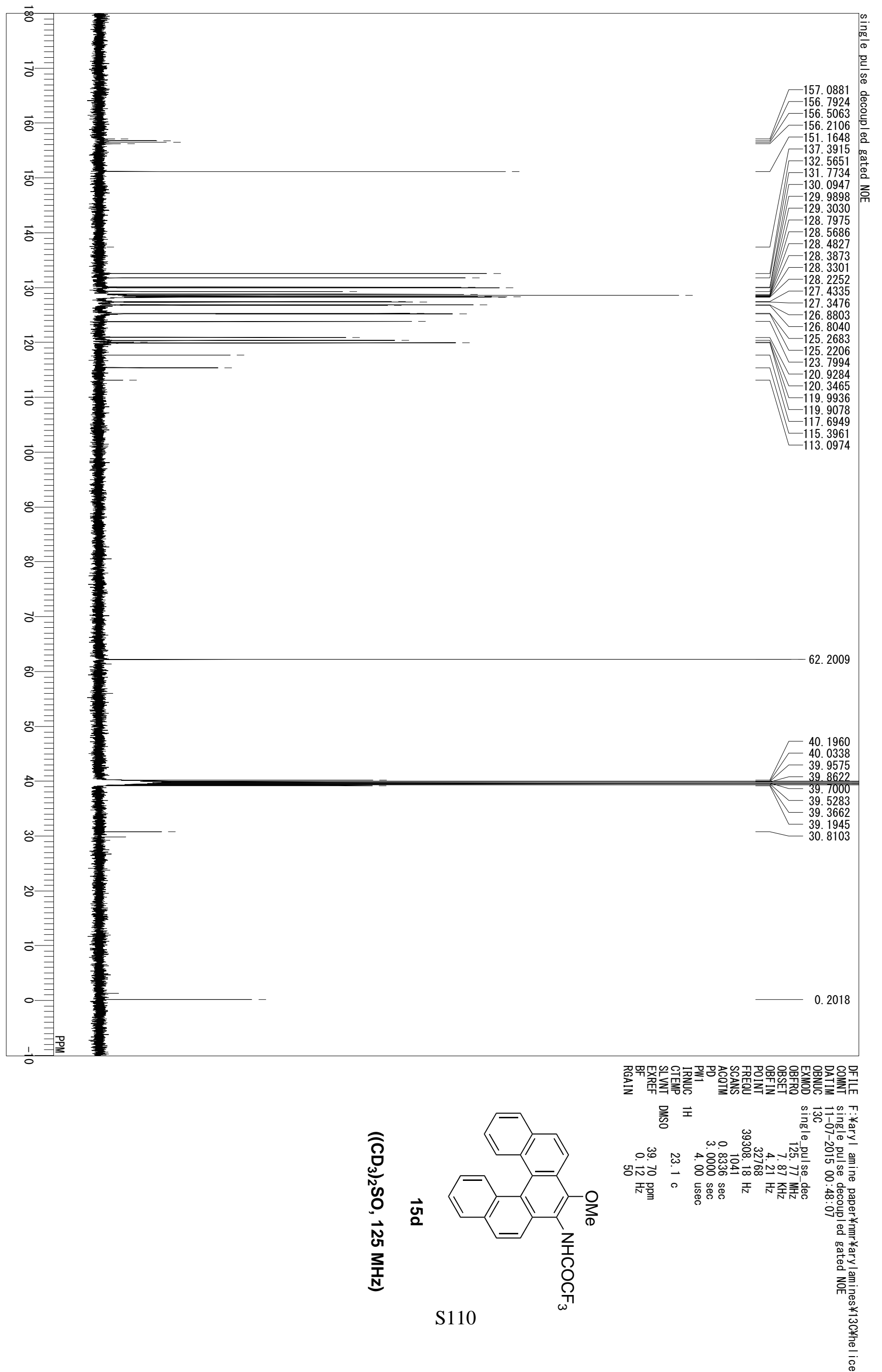




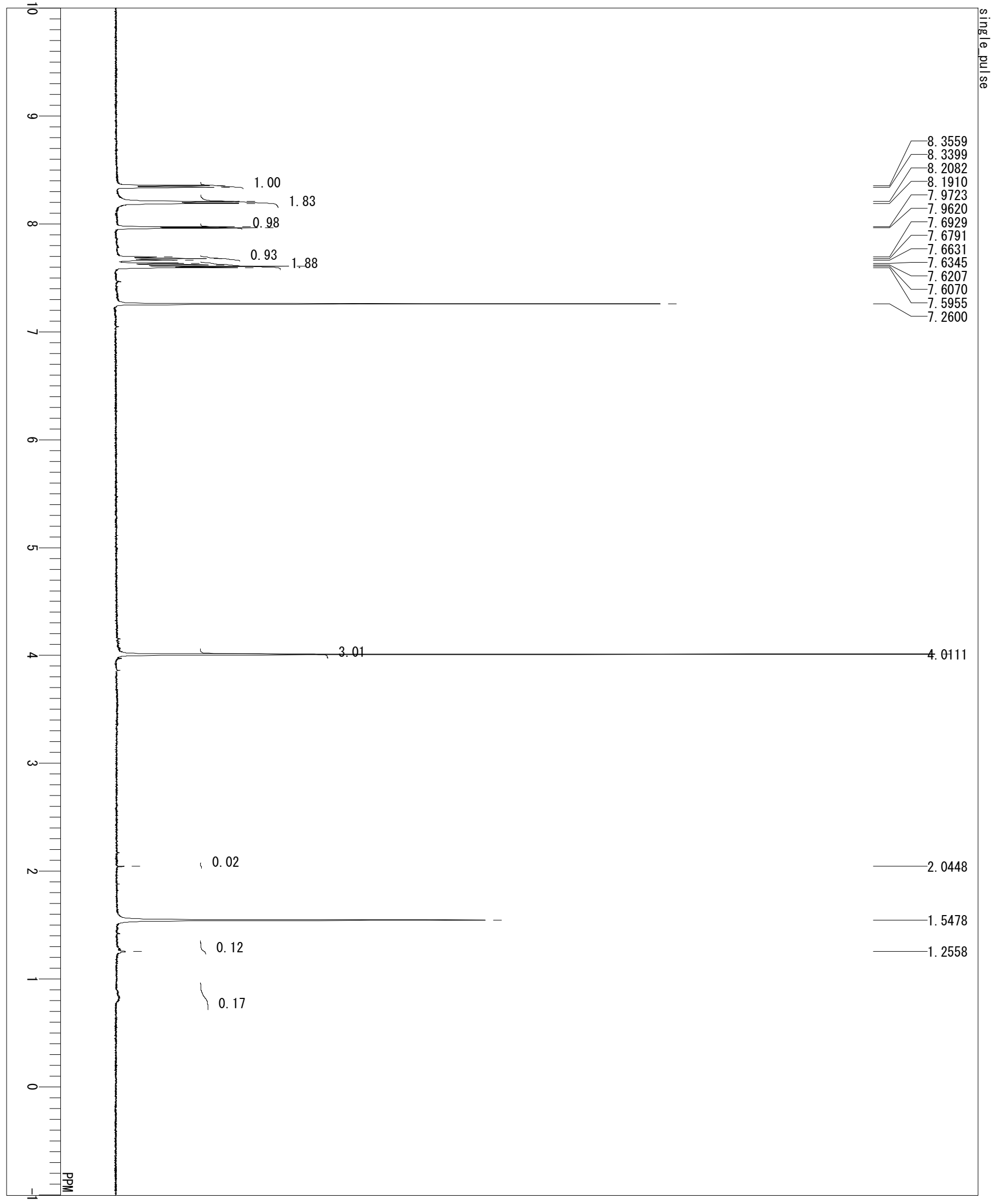

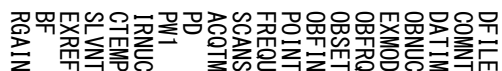

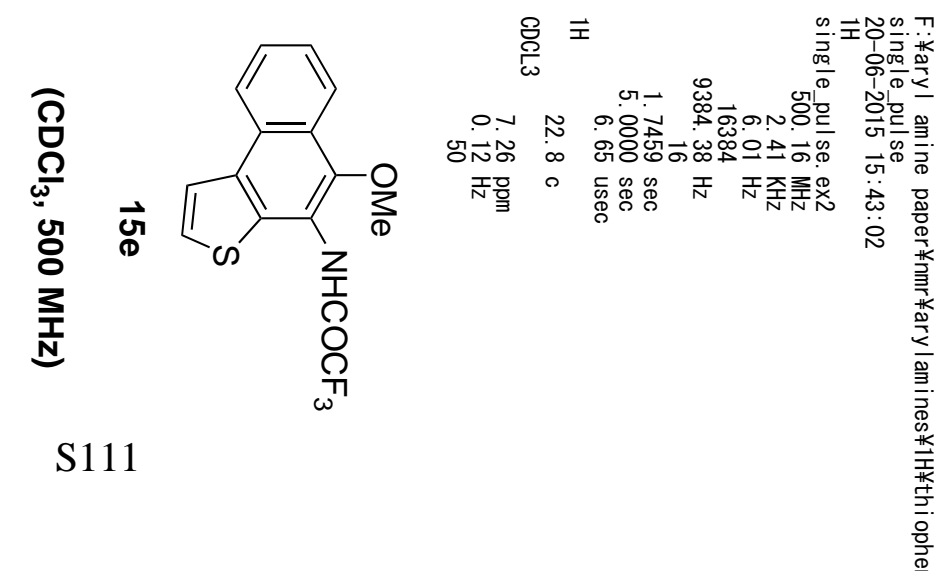




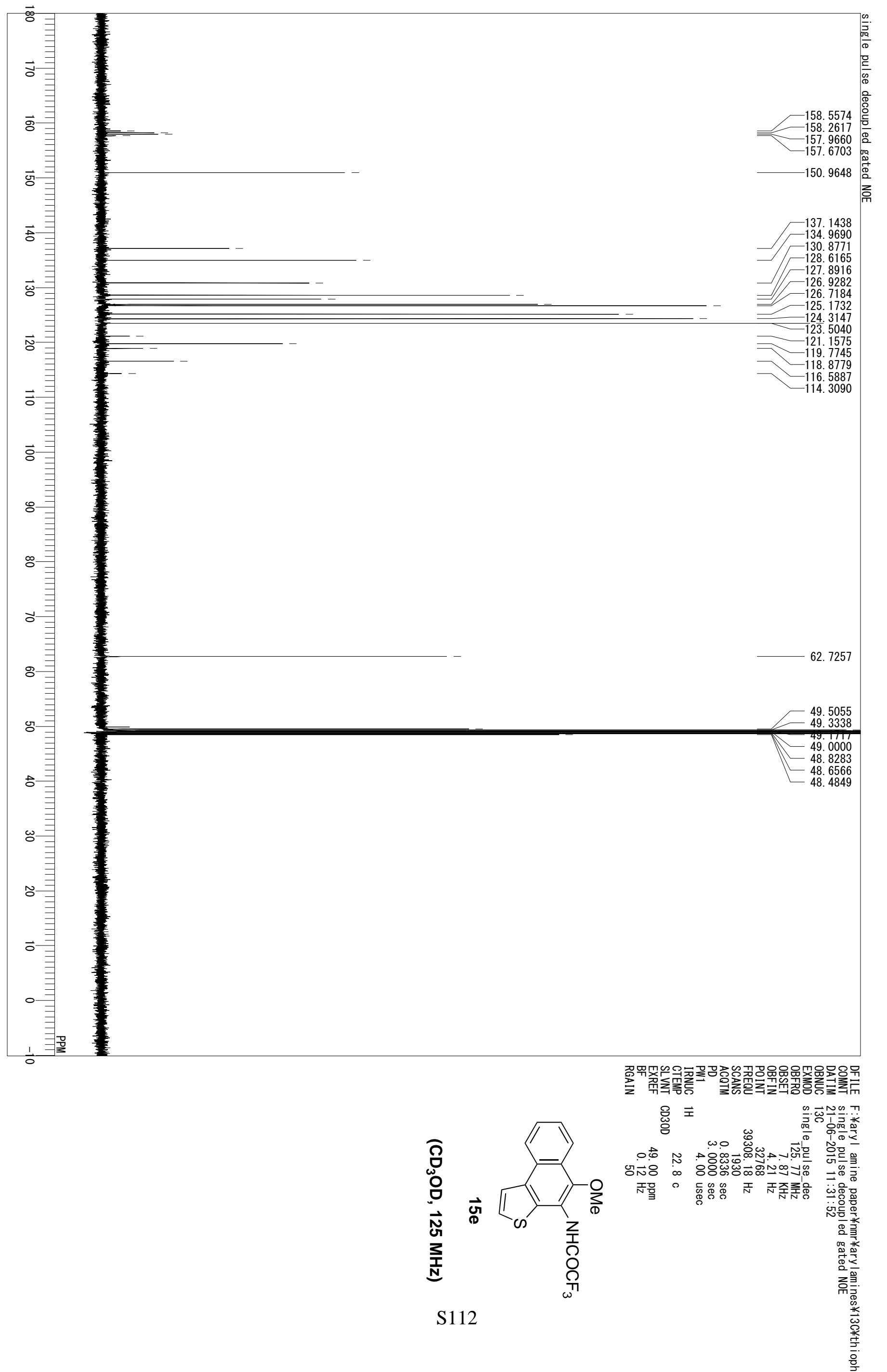




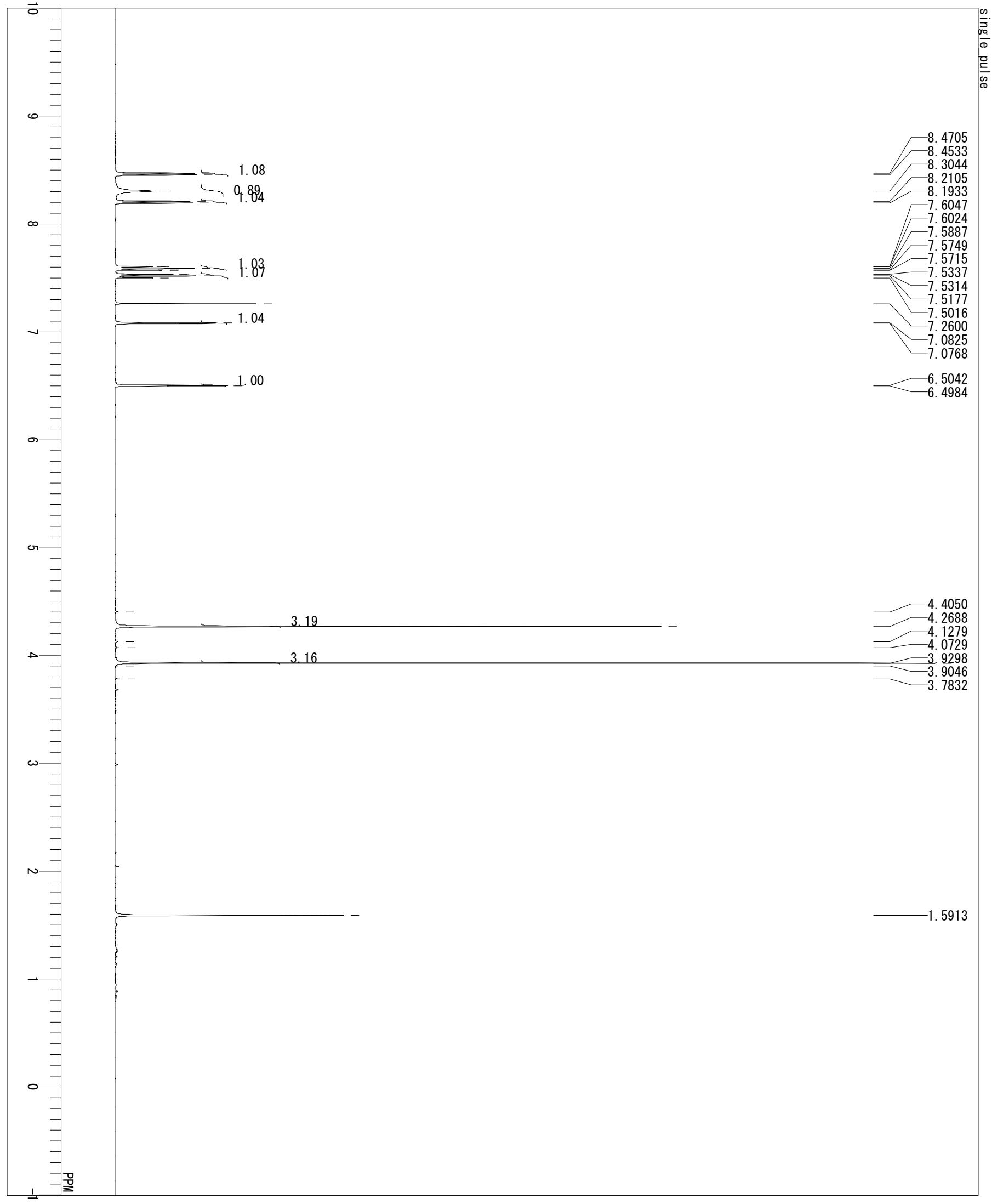

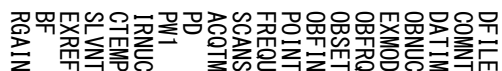

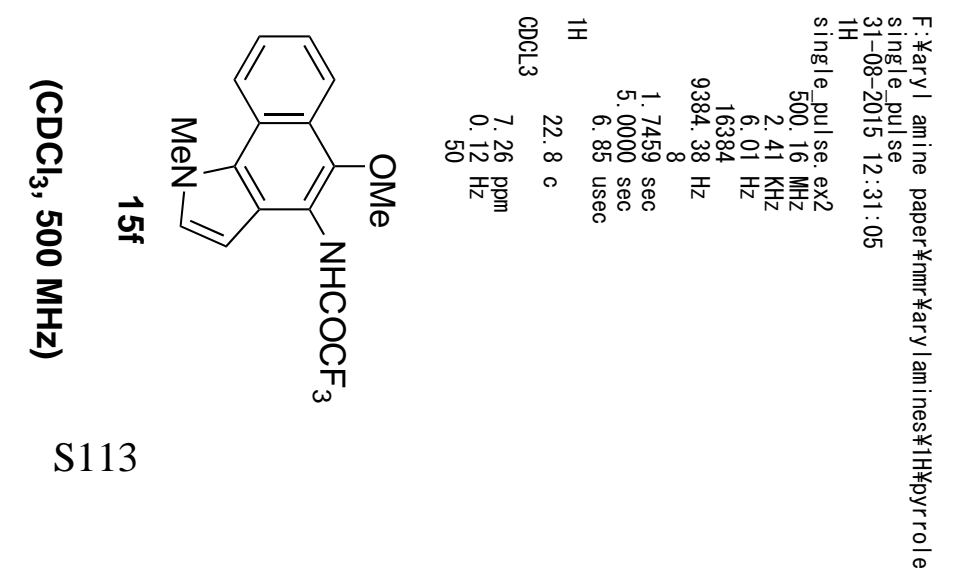




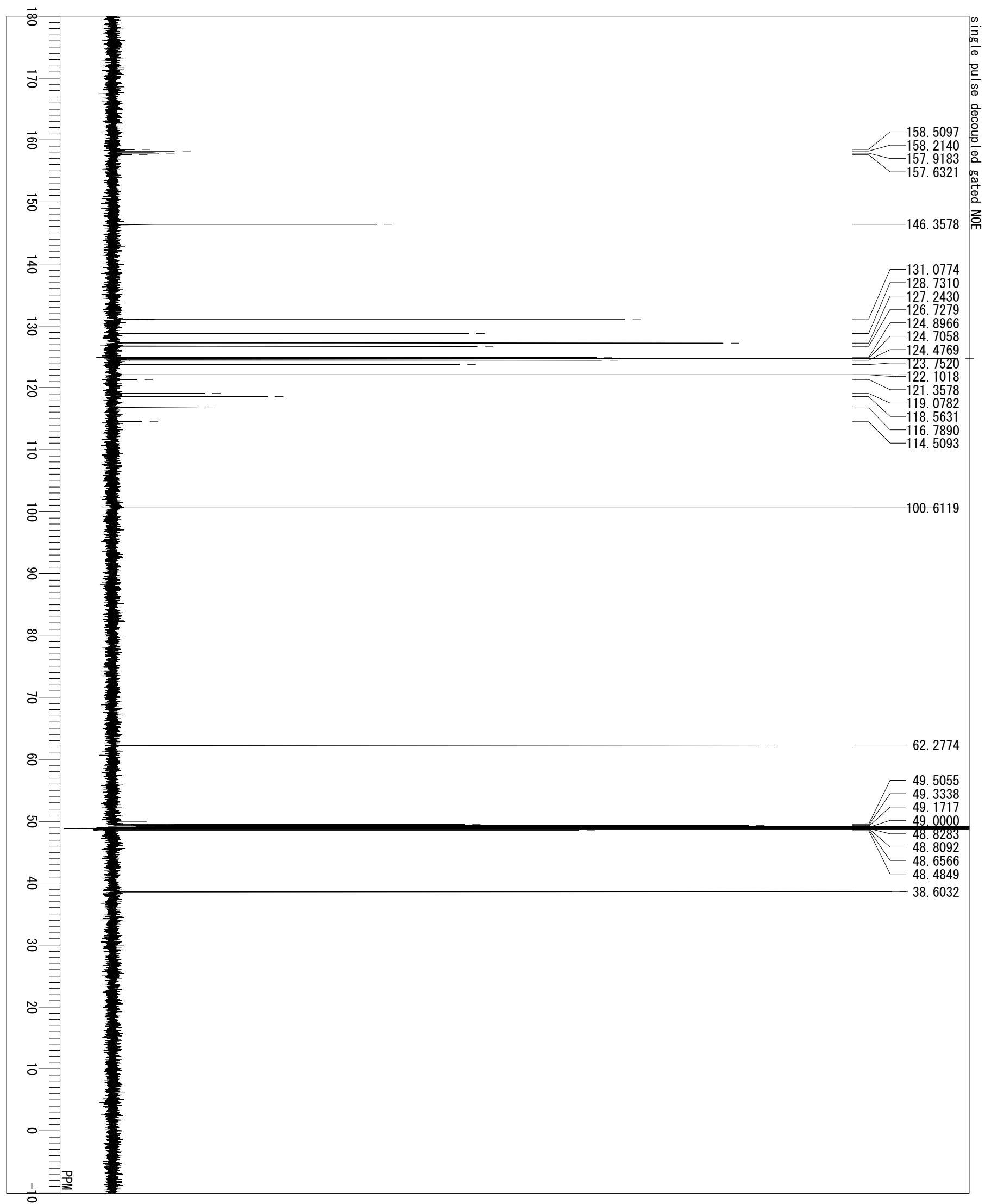

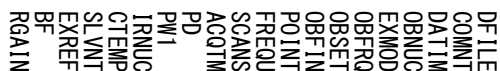

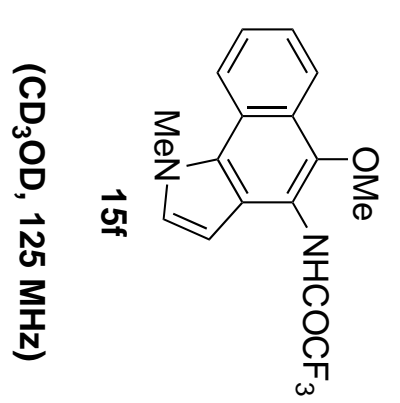

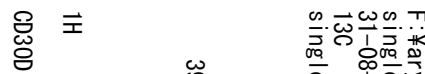

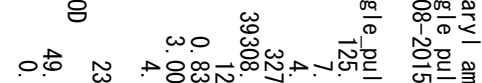
Nㅇ

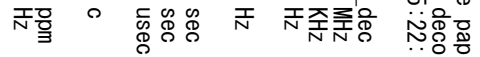



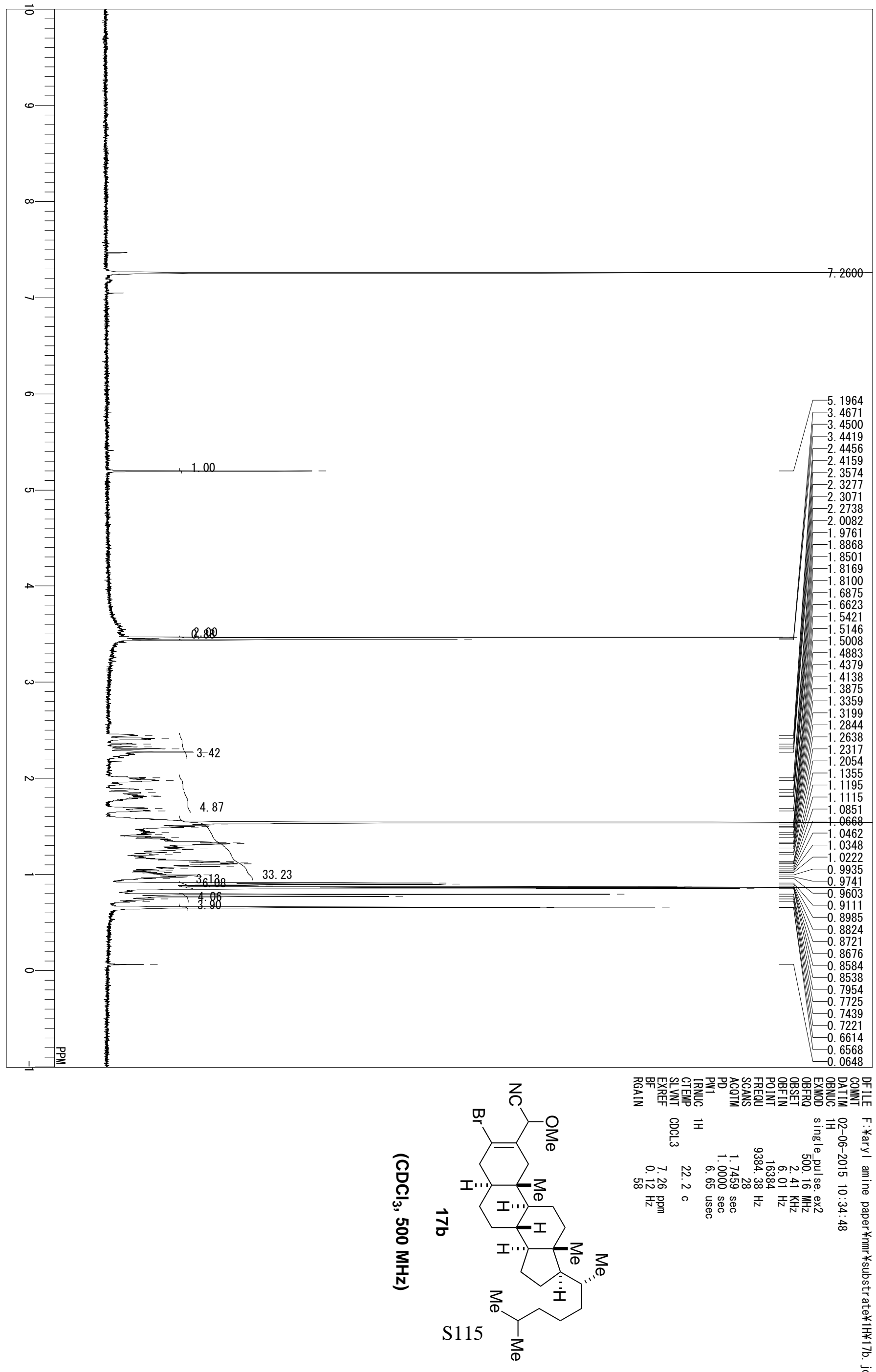

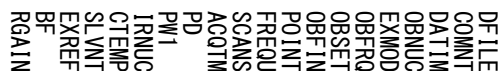

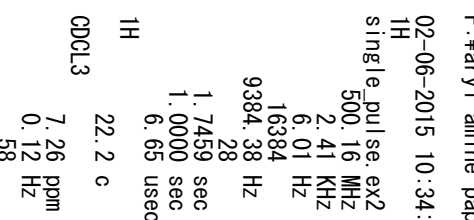

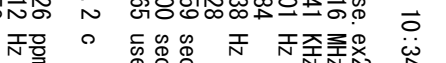
$\stackrel{\leftrightarrow}{\circ}$ 

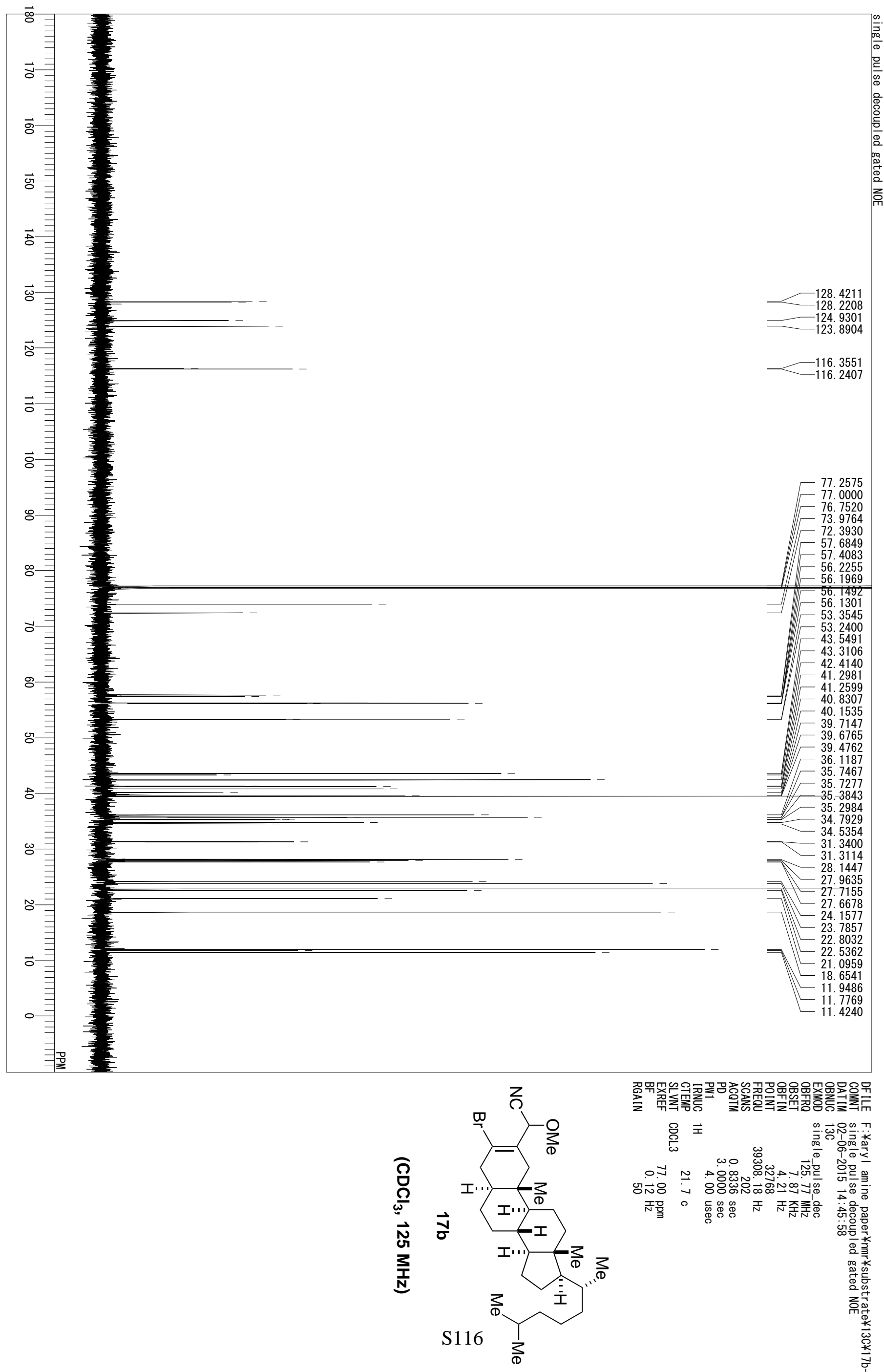

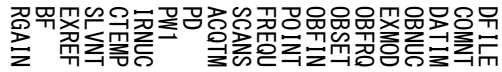

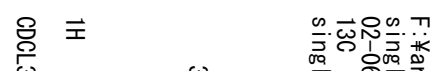
○. Giv

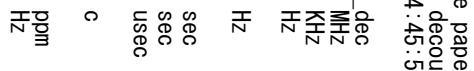

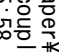




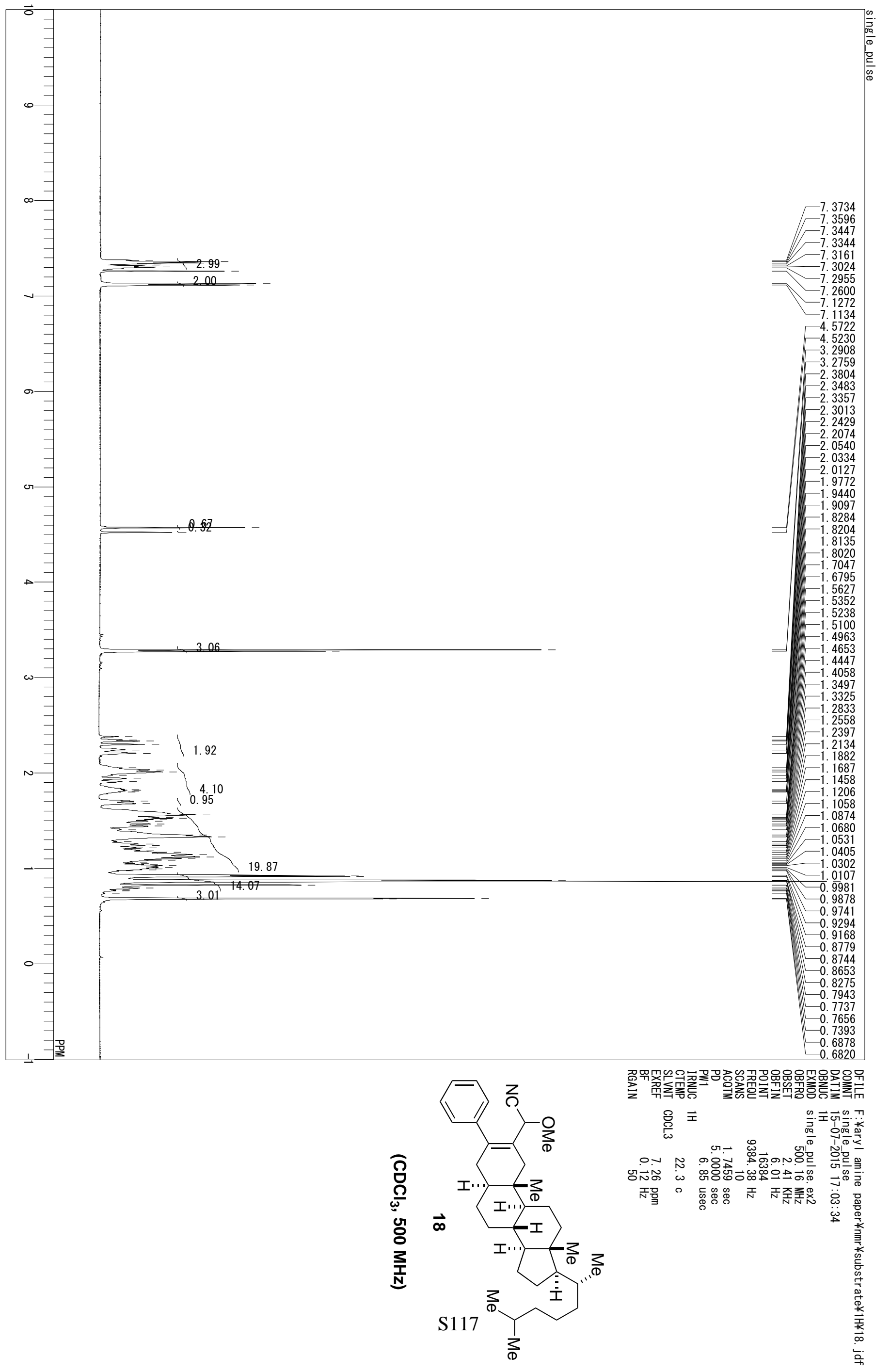



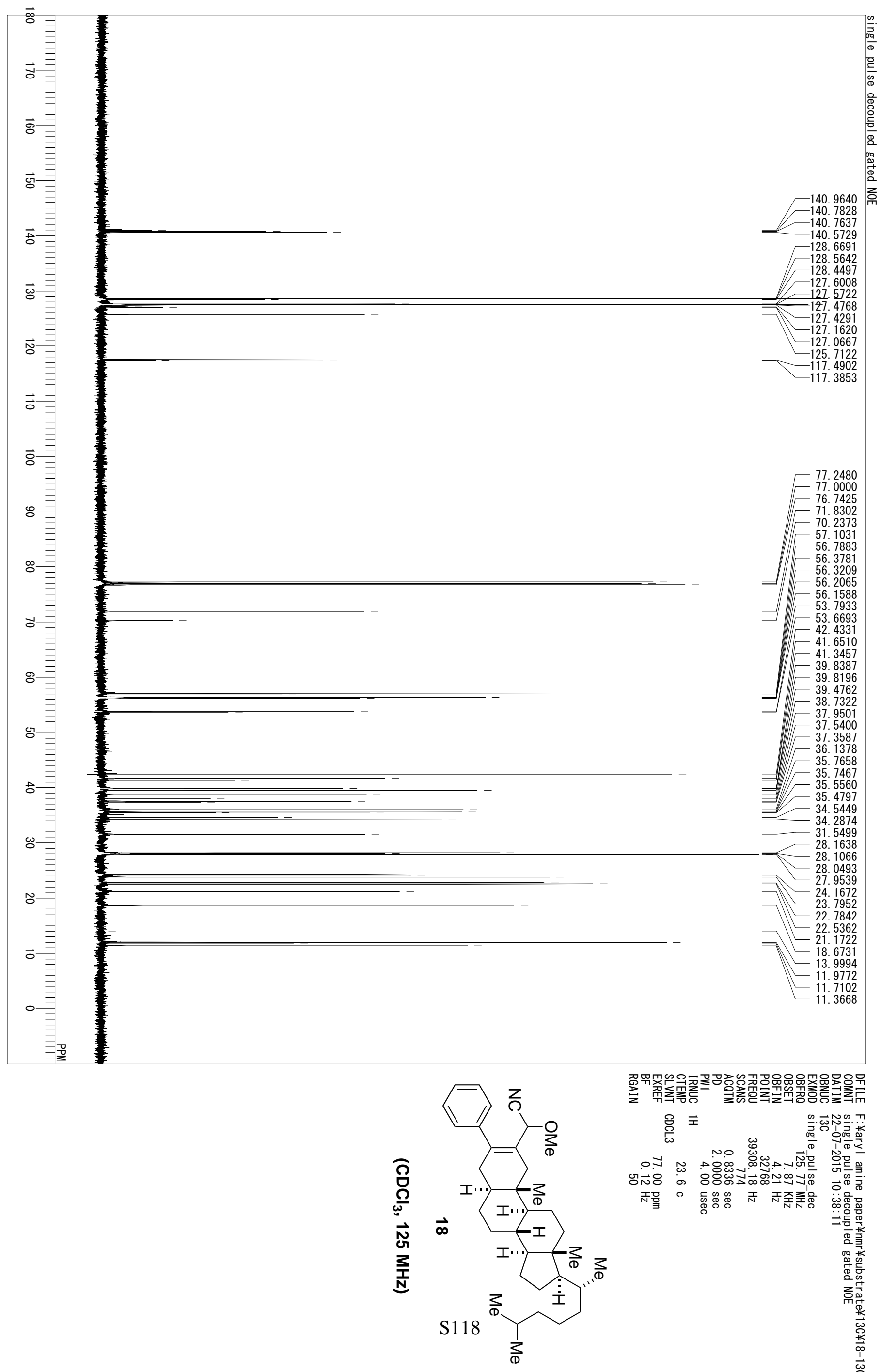

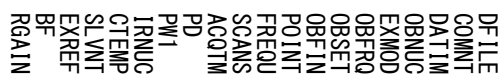

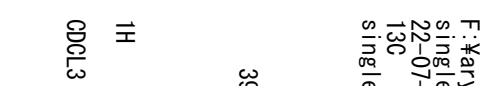

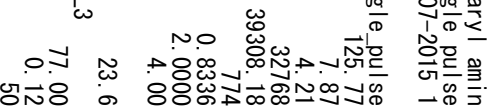

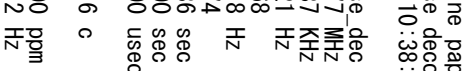
每 


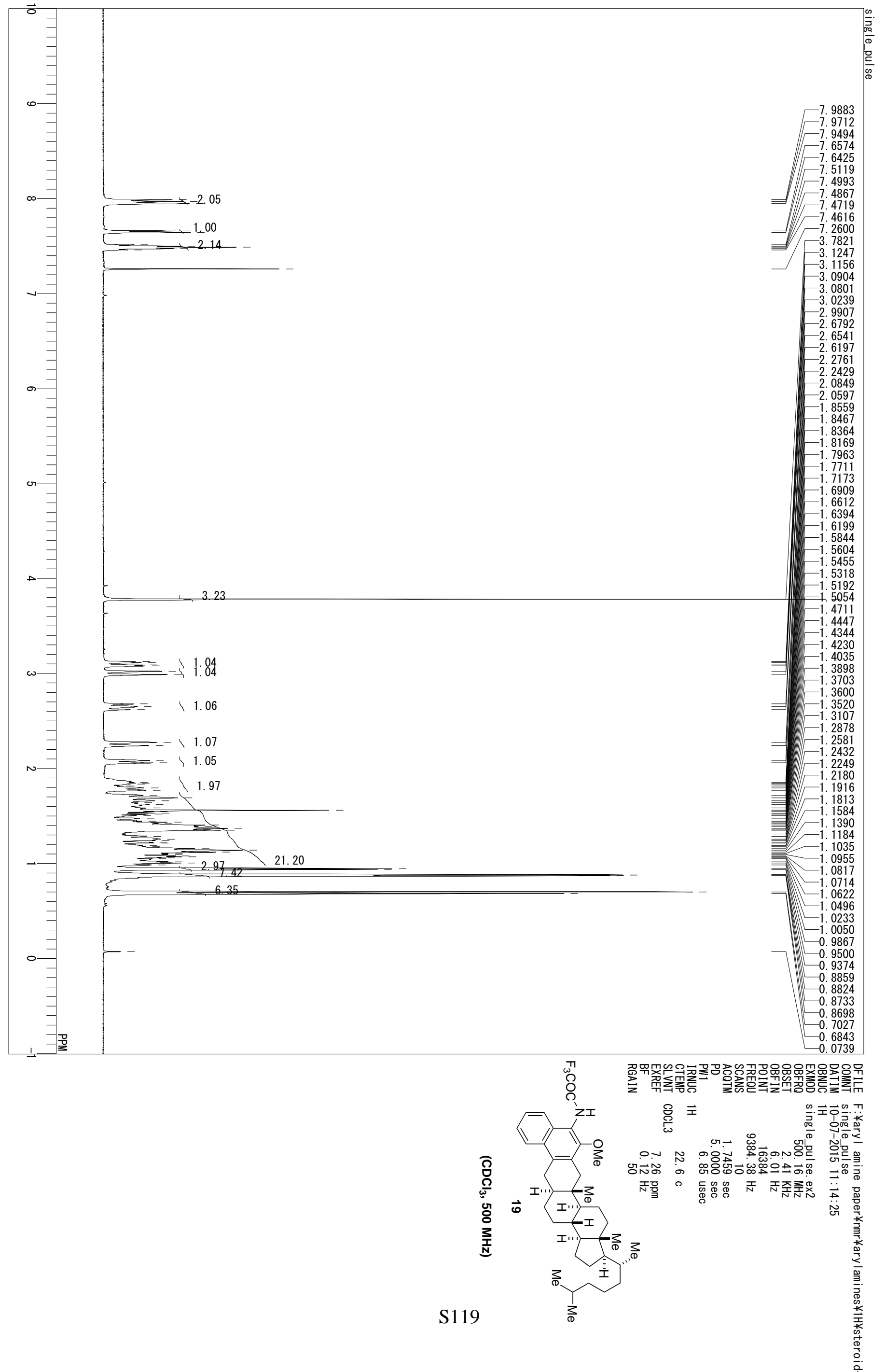



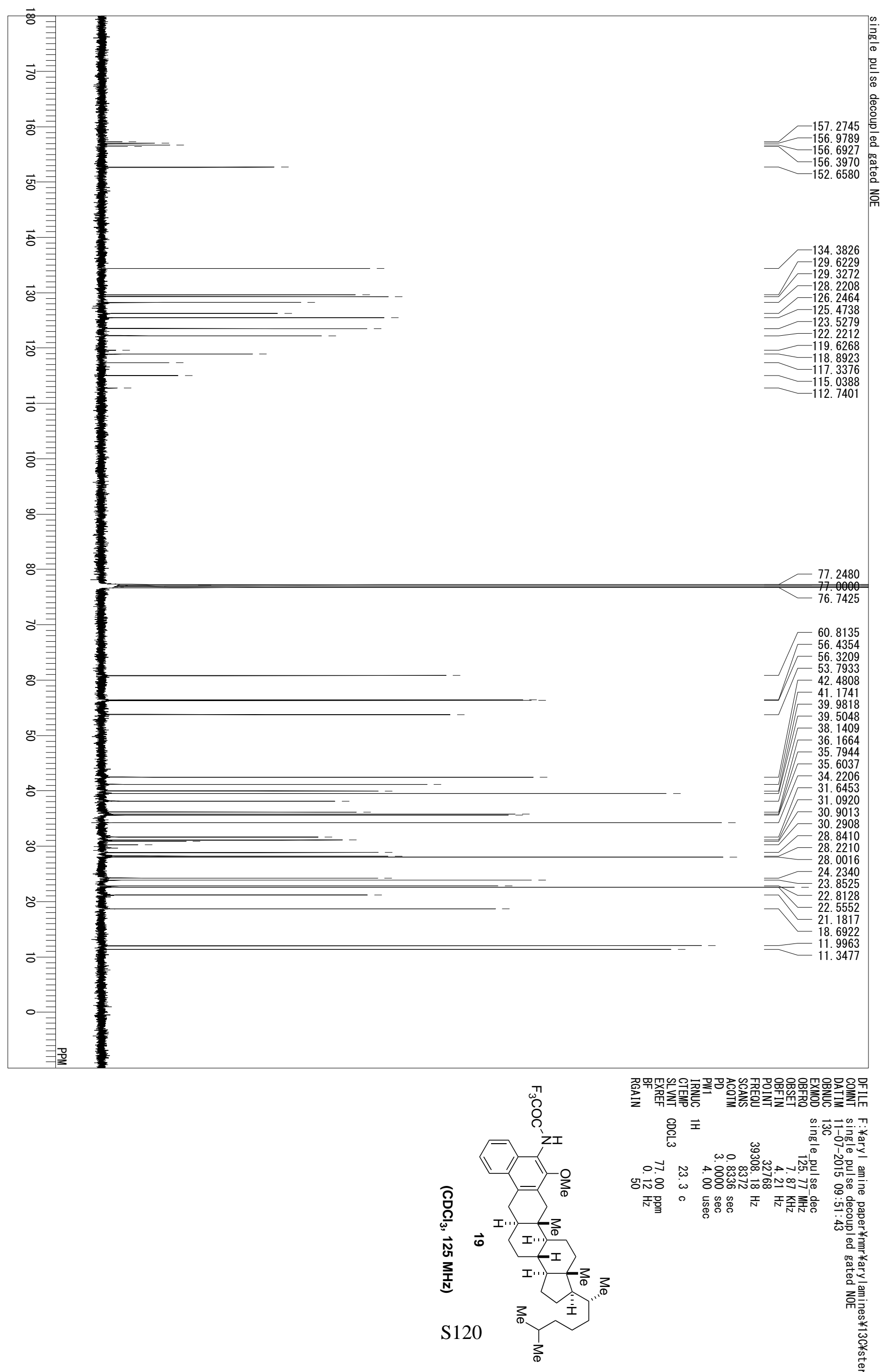

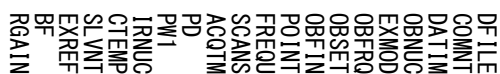

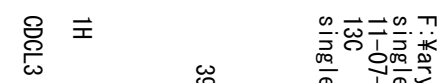

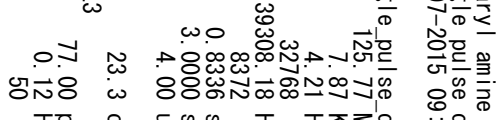

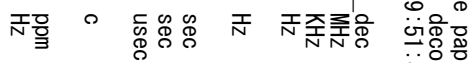




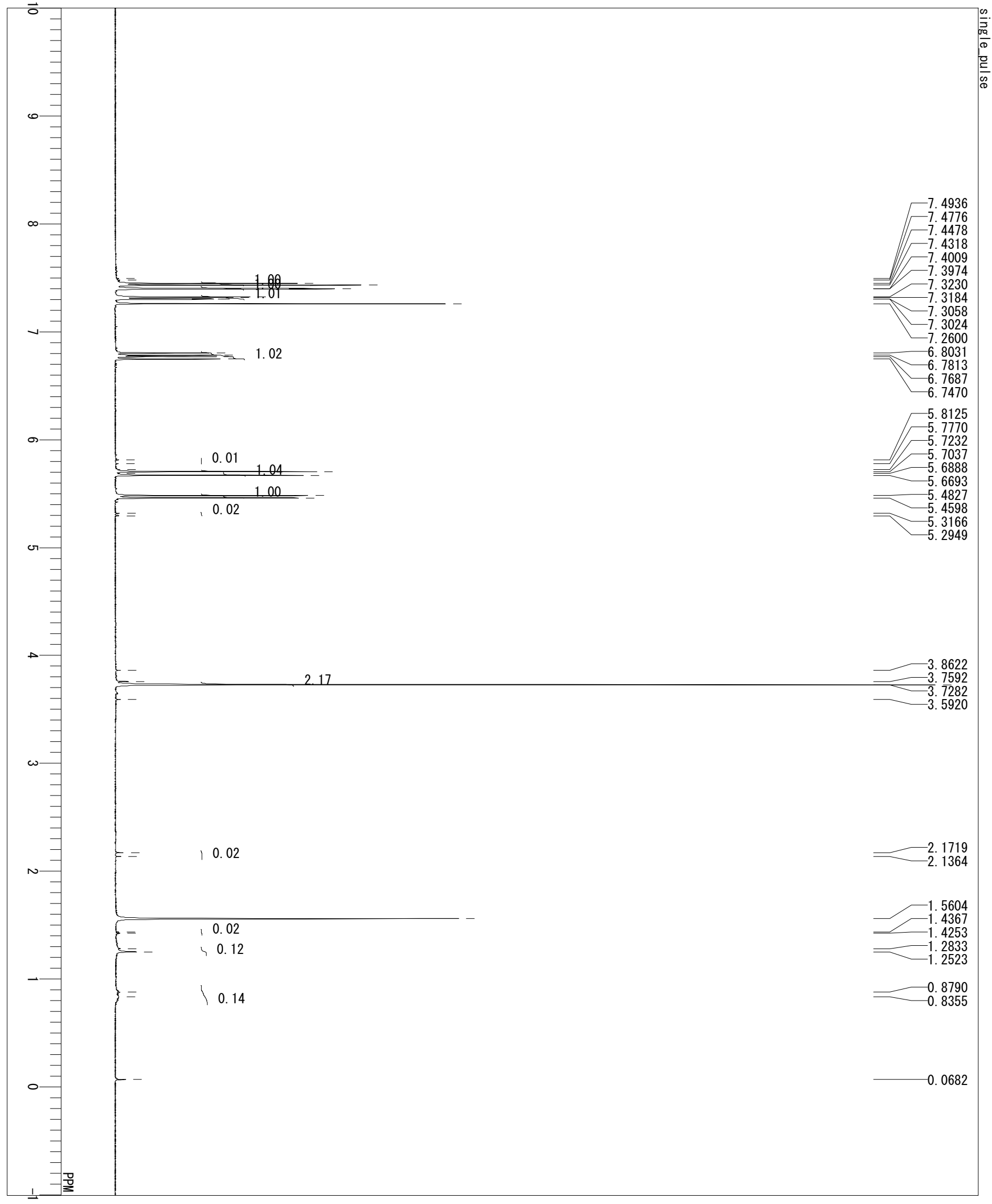

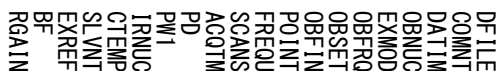

畄

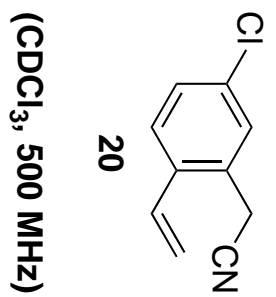

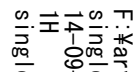

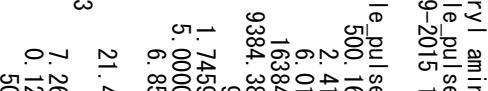

Nㅜ

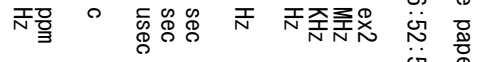

ن⿺辶 


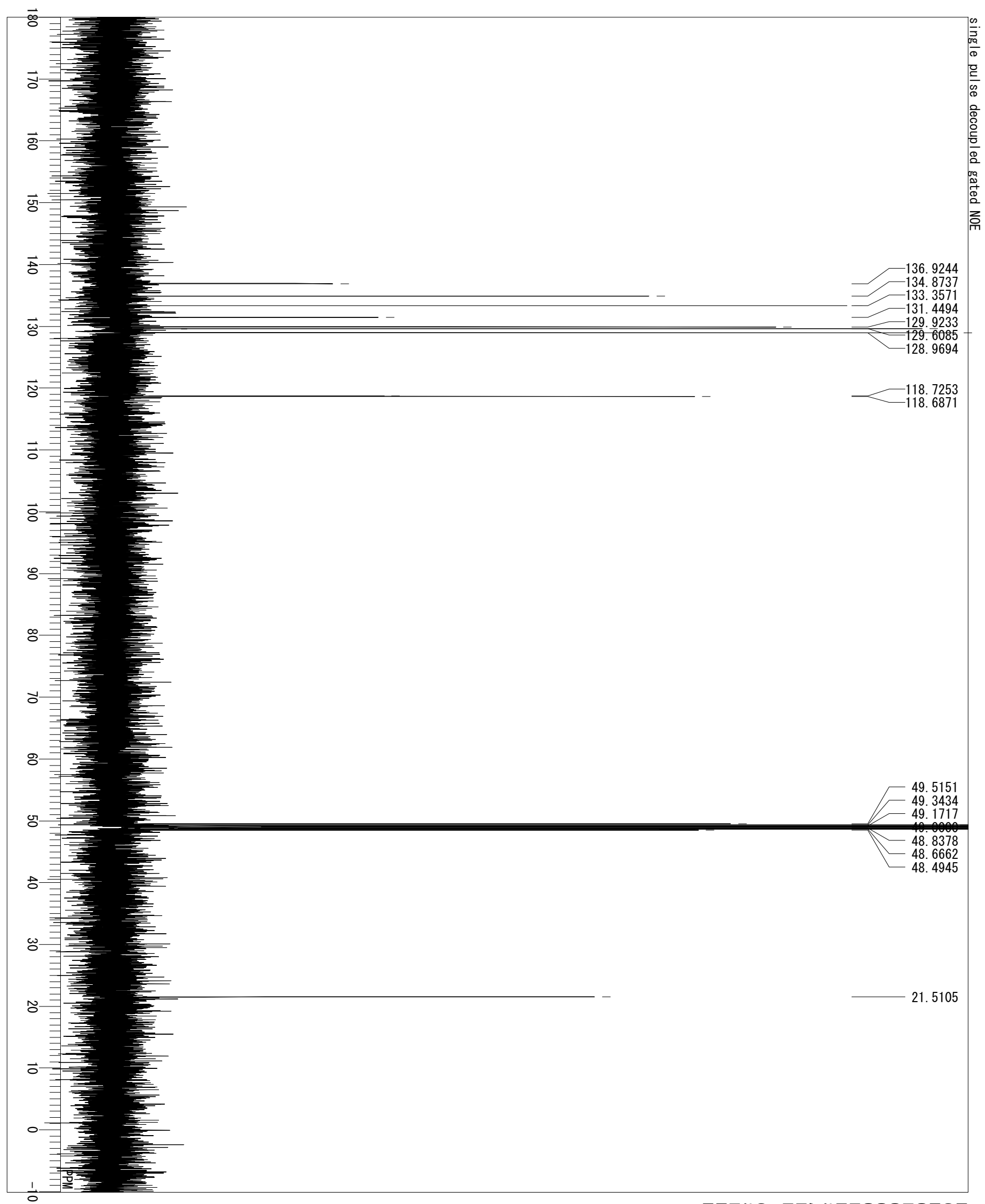

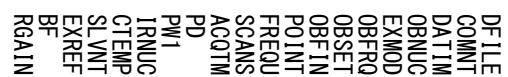

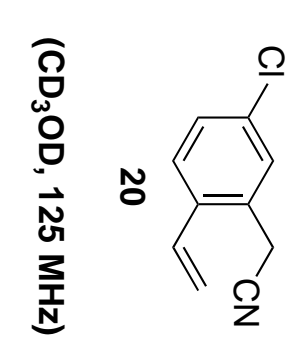

峞 $\vec{\square}$

of no 造

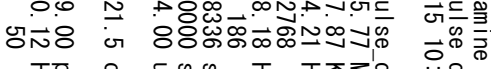

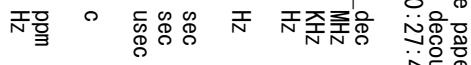

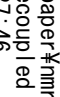

要

告

z市尔 

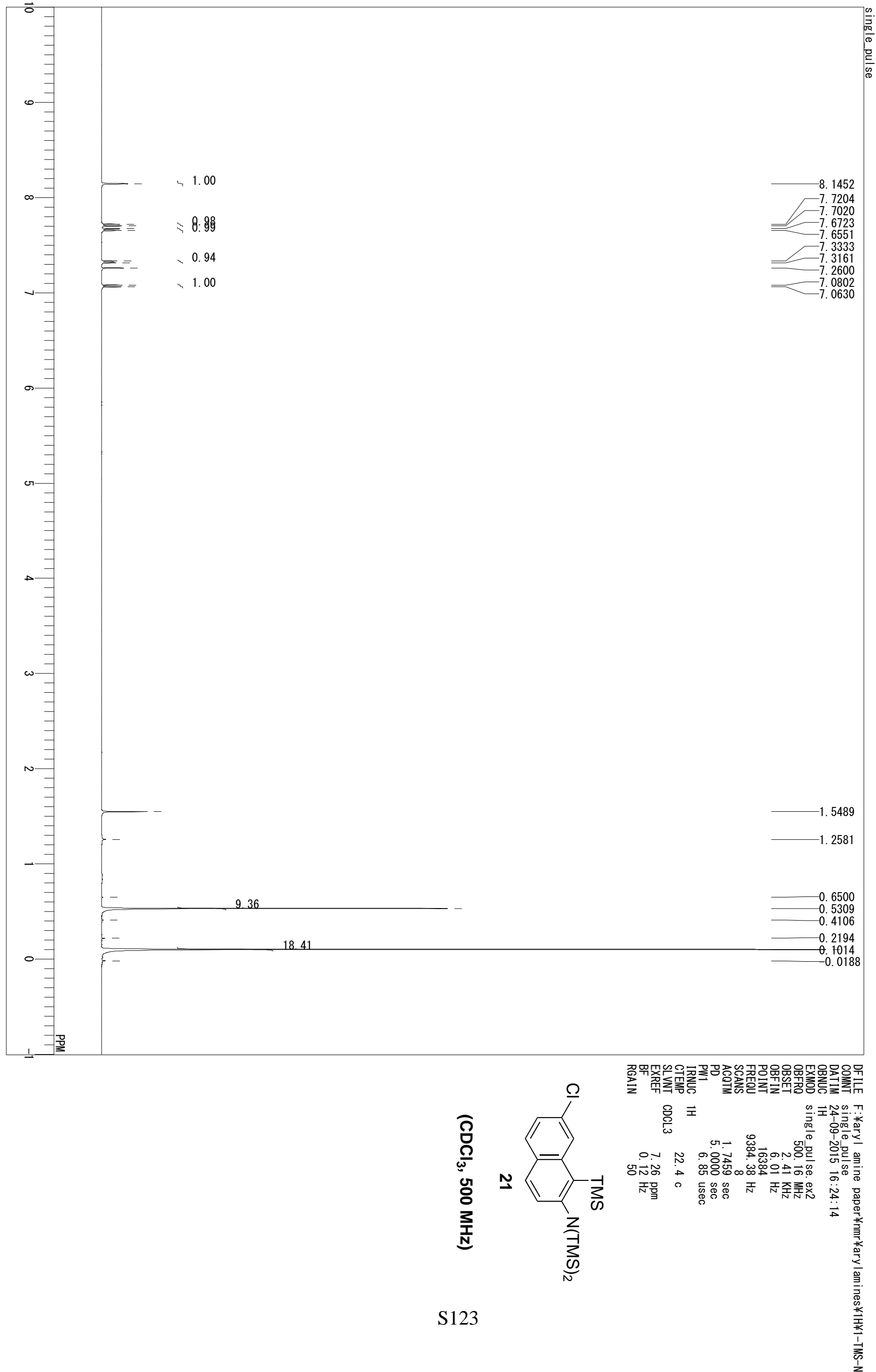


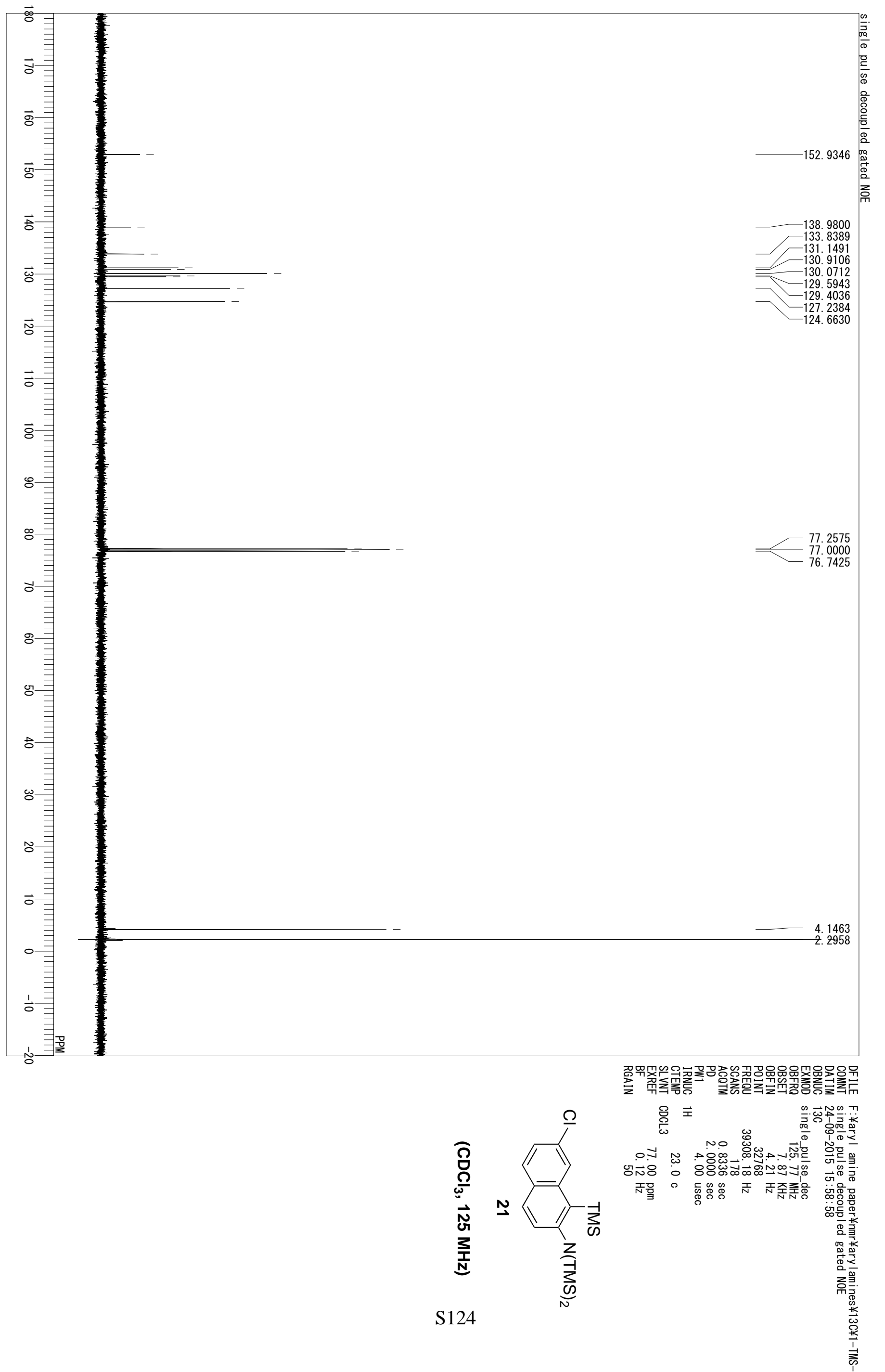




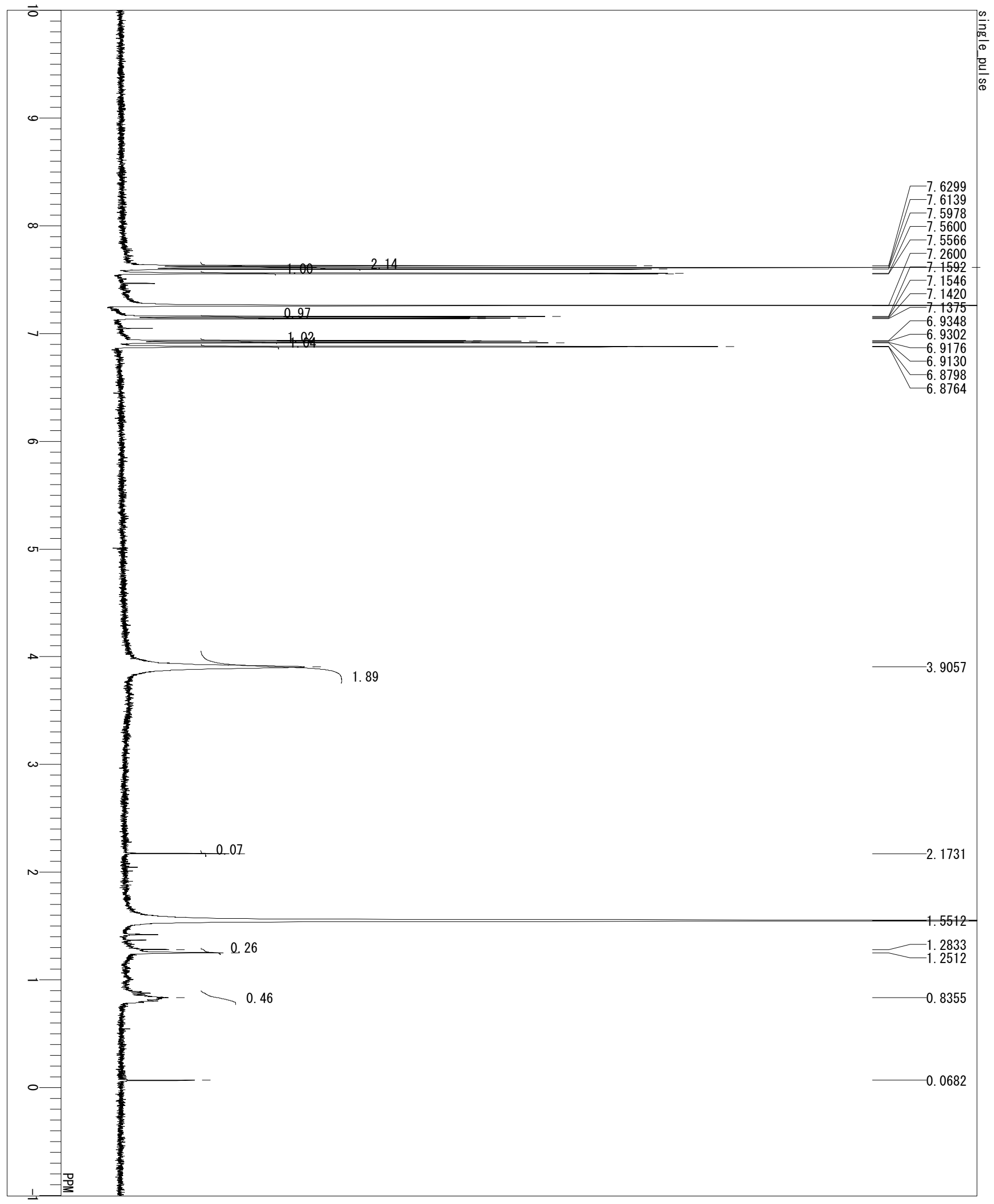

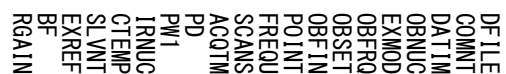

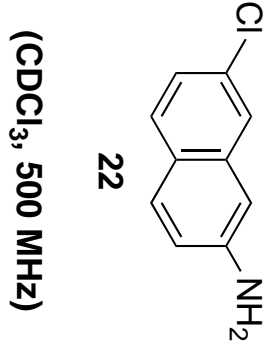

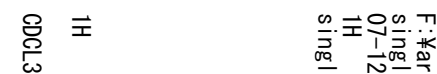

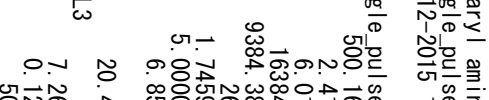
Non $\rightarrow$ जon

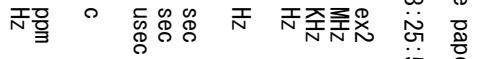
نु 


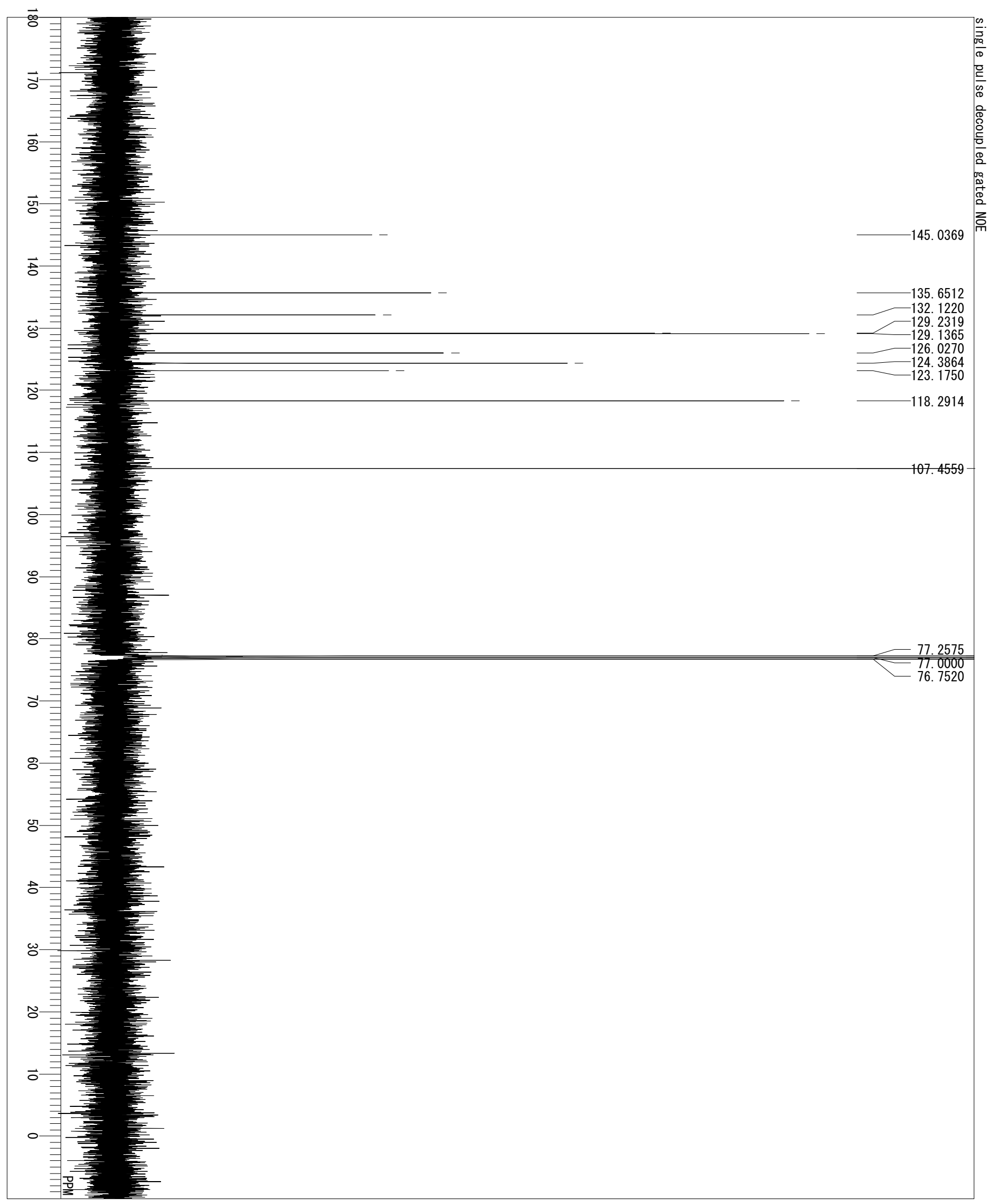

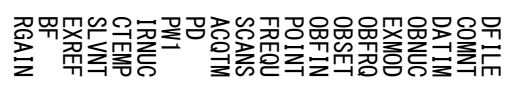

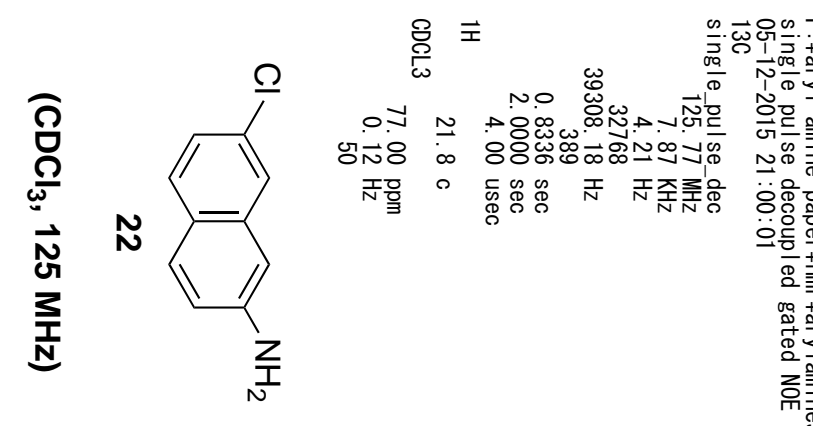




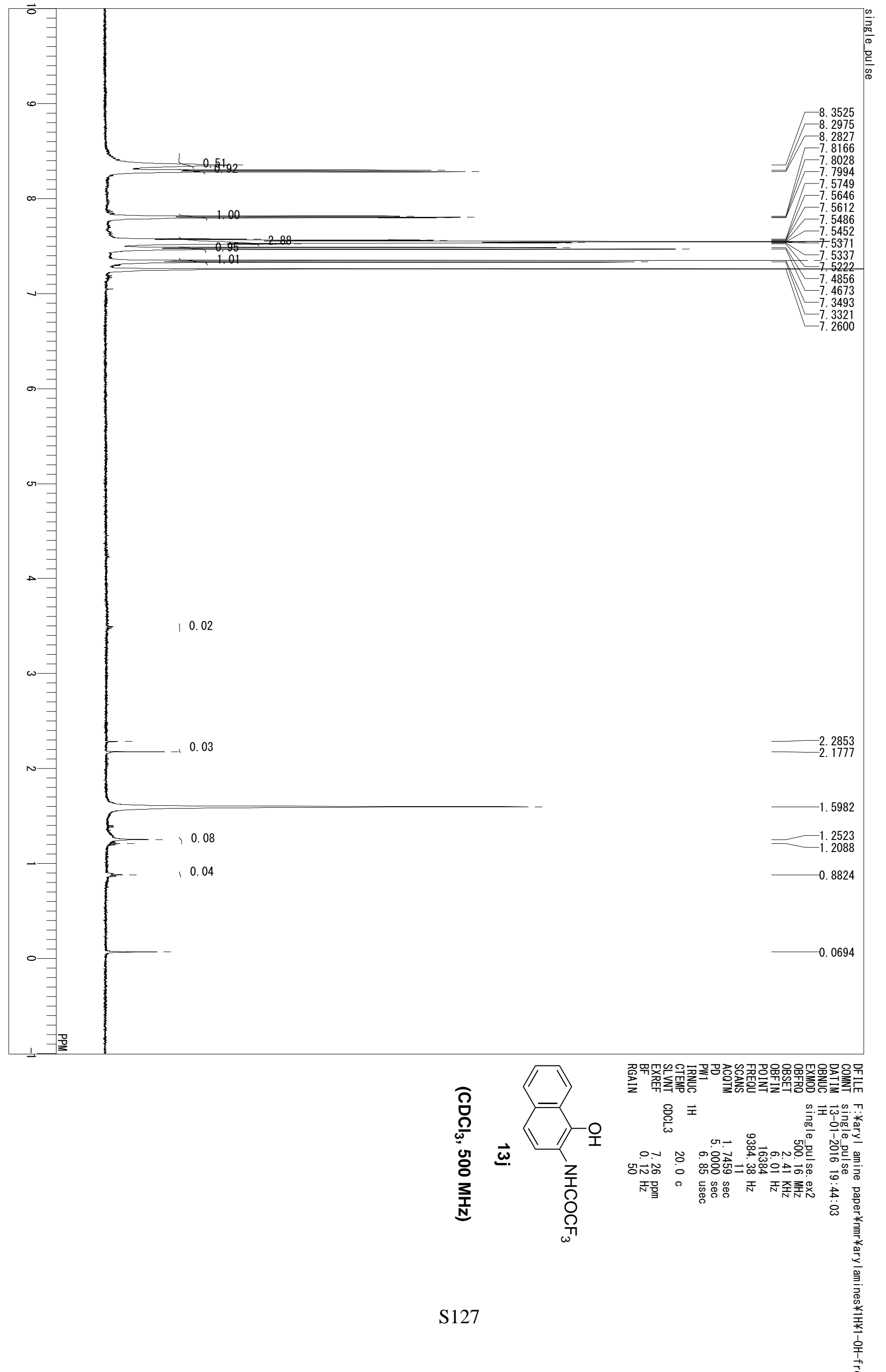




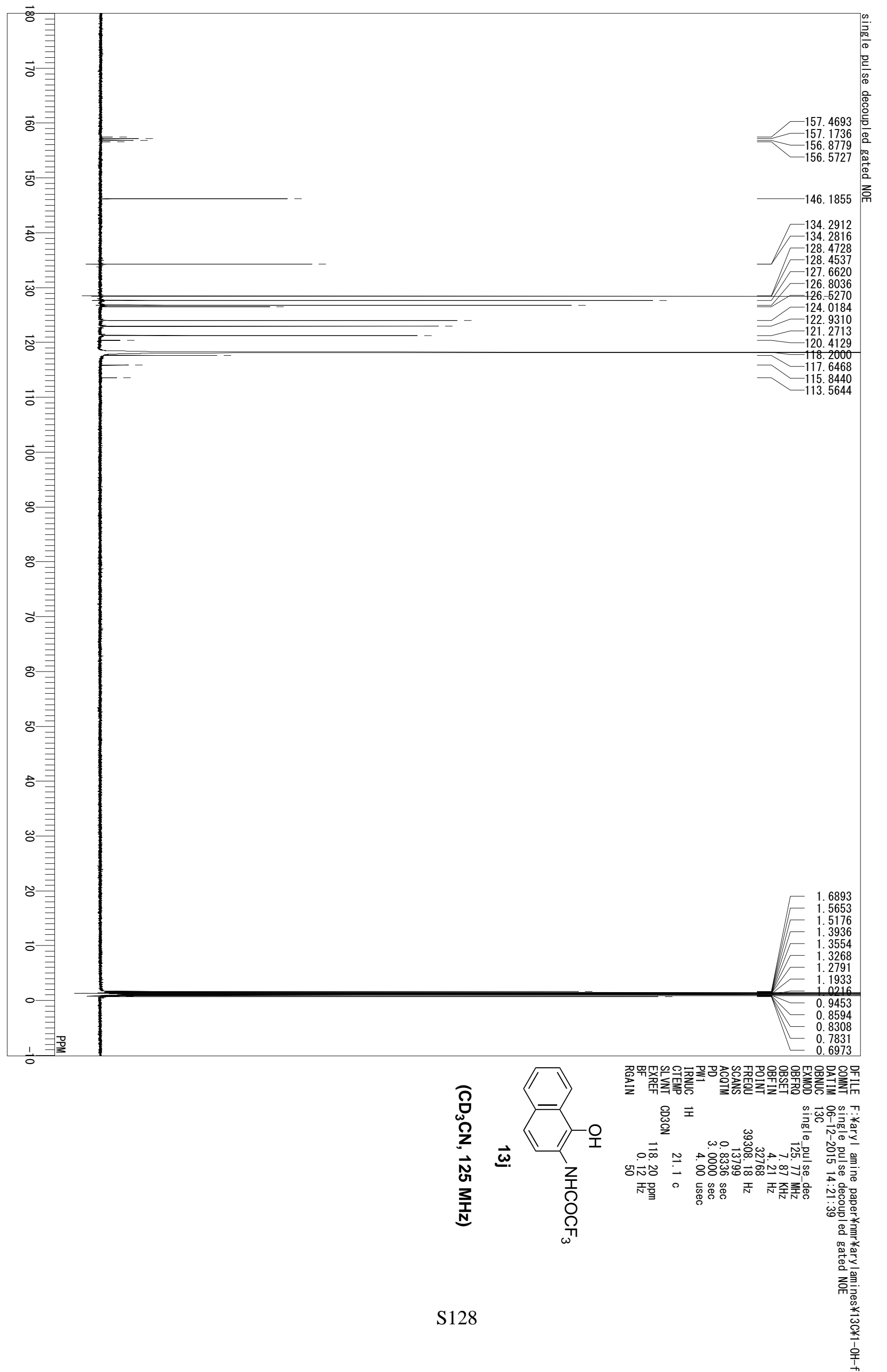




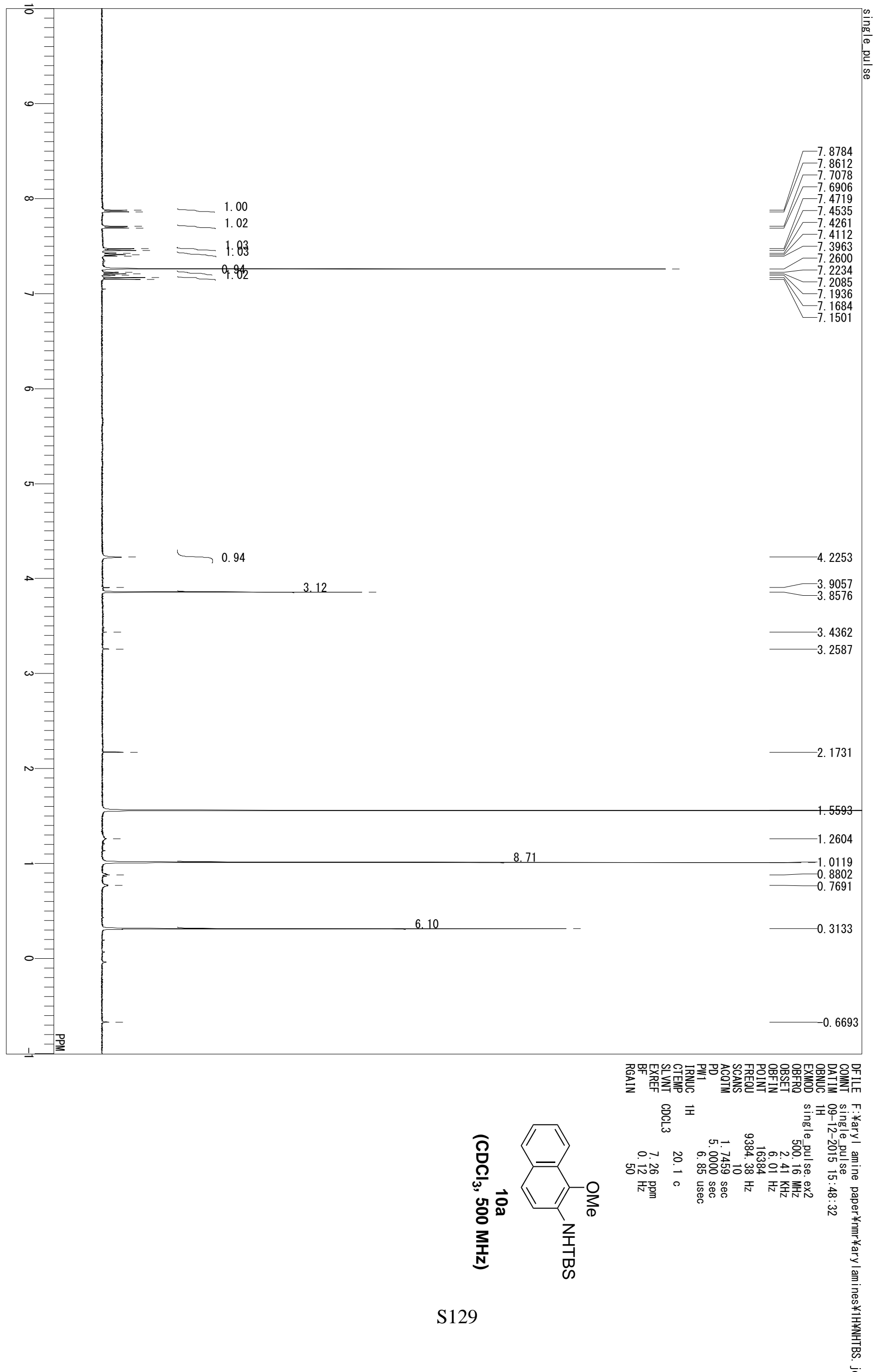




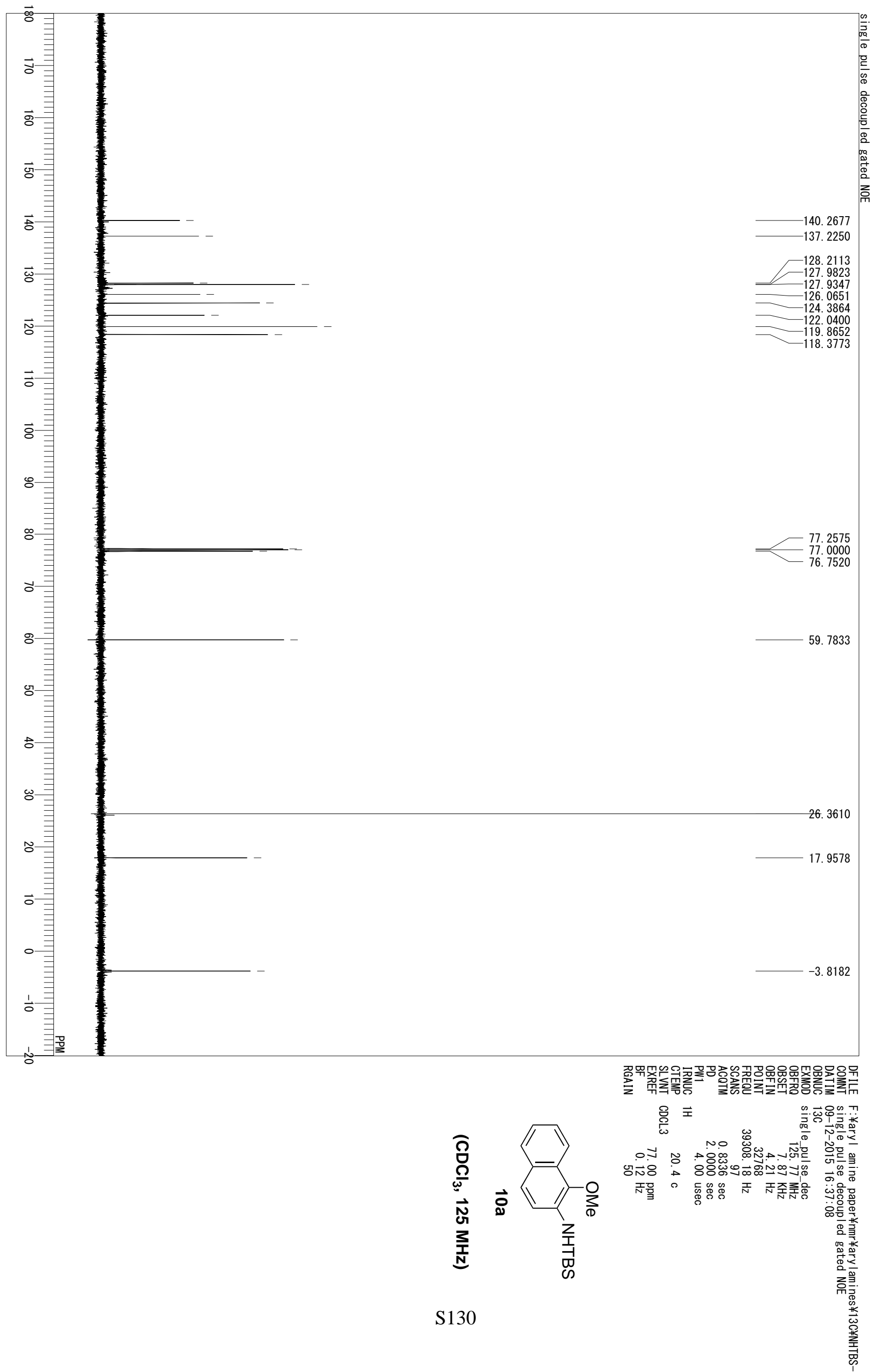




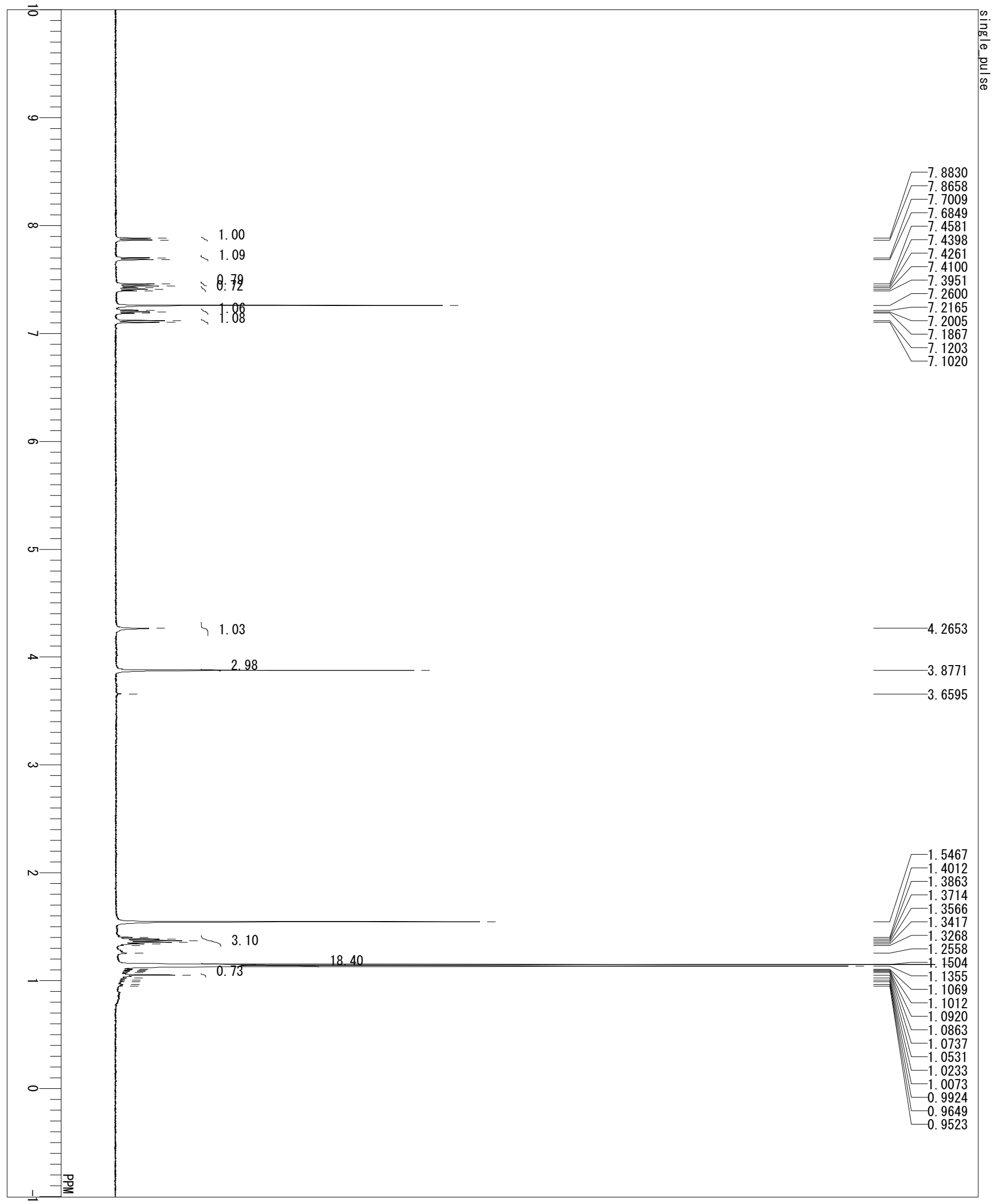

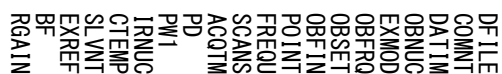

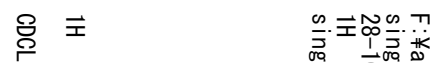

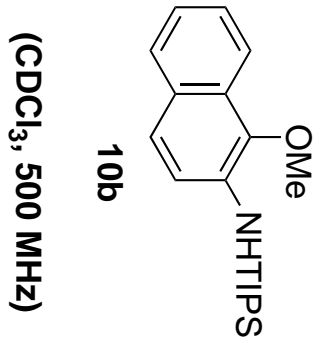
‥ N

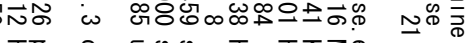

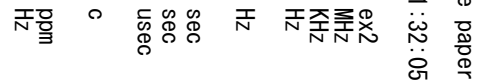




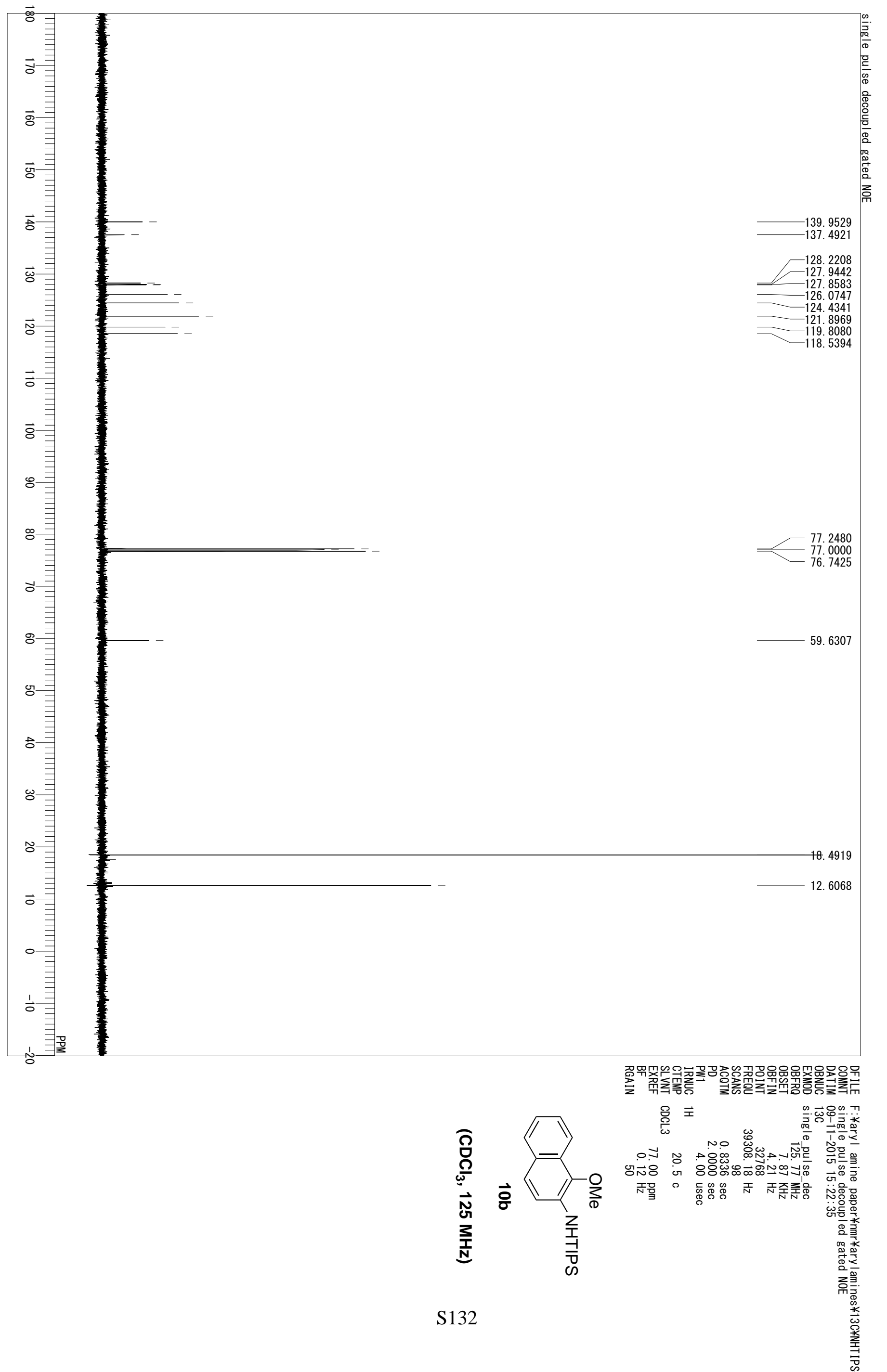

